\title{
Moisture in New \\ Zealand Bathrooms Analysing Moisture Events
}

by

Alister Stubbe

This thesis was submitted in fulfilment of the requirements of the degree for Master of Building Science to the School of Architecture, Victoria University of Wellington.

Victoria University of Wellington 


\section{Moisture in New Zealand Bathrooms}

\section{Preface}

\section{Author}

Alister Stubbe

School of Architecture

Victoria University of Wellington

Email: alister.stubbe@gmail.com

\section{Supervisor}

Nigel Isaacs

Senior Lecturer

School of Architecture

Victoria University of Wellington

Email: nigel.isaacs@vuw.ac.nz 


\section{Abstract}

A literature review was carried out on the impact of moisture in New Zealand homes as well as the role ventilation and occupant behaviour play in controlling this. Bathrooms in residential homes were identified as being especially vulnerable. NZS4303:1990, clause G4 Ventilation of the New Zealand Building Code, and clause E3 of the New Zealand Building Code were summarised to provide context for how New Zealand buildings are designed. Measurements taken in houses throughout New Zealand by BRANZ as part of the House Condition Survey were made available for analysis. This included measurements of relative humidity and temperature.

Data from one Dunedin house was thoroughly explored. This involved three objectives. The first step focused on identifying periods of rapid change in the amount of moisture introduced to the indoor environment, measured in absolute humidity. These periods were named 'moisture events'. The second objective was to visually communicate the changes in temperature and absolute humidity taking place on individual days, highlighting moisture events. The third objective was to analyse the identified moisture events, finding the key areas to focus on for the full analysis as well as areas that could be explored in further research. This process was then applied to all remaining houses.

Moisture events were grouped into four categories: increases, decreases, episodes, and combinations. Episodes were the focus of the analysis, representing moisture being actively introduced to the indoor environment and then removed. These categories were further filtered, identifying the moisture events were most likely to have had a large impact on the indoor environment. Days were broken into four hour periods, with the filtered moisture events taking place in each period recorded. These were used to identify patterns in moisture events for each house. If a certain pattern of moisture events frequently took place, then days containing that pattern were described as a 'typical day' for that house. The mean and median absolute humidity at the start, peak, and end of the unfiltered episodes from each house were then calculated. The mean and median episode length was also calculated. The results were compared to the Household Energy End-use Project (HEEP) and to the typical days for each house. The results were grouped according to factors such 
Moisture in New Zealand Bathrooms

as the number of bathrooms in the house, the floor area, the house location, and the event length.

The number of bathrooms present in the house was found to have a large impact on the size and frequency of moisture events. As expected, larger bathrooms recorded lower increases in absolute humidity from the start to the peak of episodes. Rooms with a greater volume would require more moisture to reach the same number of grams of water per cubic metre. However, the smallest bathrooms also recorded low increases in absolute humidity. 


\section{Acknowledgements}

The past year I spent working on this thesis has been one of the most fun, difficult, and worthwhile experience of my life. Thank you to everyone who supported me and made this possible. I would especially like to thank my supervisor Nigel Isaacs, senior lecturer at the Victoria University of Wellington School of Architecture, for the extraordinary assistance he has provided me over the past year, going above and beyond to help me. I could not have finished without his guidance and input.

I am grateful to BRANZ for allowing me to use the data they collected. In particular I would like to thank Manfred Plagmann, senior physicist at BRANZ, for all the help he provided, inspiring the topic of my research and giving me feedback. I would also like to thank Vicki White, research scientist at BRANZ, whose work informed a large part of my thesis and who responded to my (many) sudden requests for more data.

Finally, I would like to thank my friends and family for their constant support. To my family, thank you for all the advice you gave me and helping me relax when I needed to step back from my work. I would especially like to thank my mum, Phillipa, who edited my work, gave me feedback, and was always willing to lend a hand. To my partner Hilary, thank you for always being there for me. To my friend and flatmate Tim, thank you for putting up with me. To my fellow MBSc students, the fact we are all still on speaking terms after everything we went through over the past five years is a miracle. You were the best team I could have hoped to work with. I consider myself extremely fortunate to be friends with each of you. 


\section{Contents}

Moisture in New Zealand Bathrooms

Preface

Abstract.

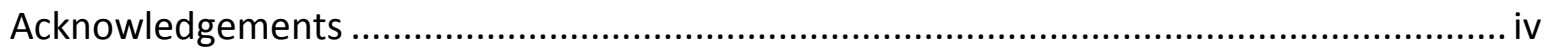

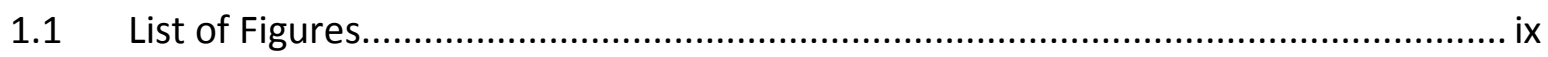

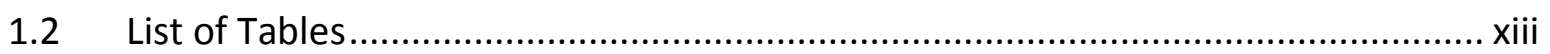

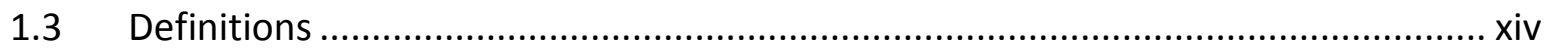

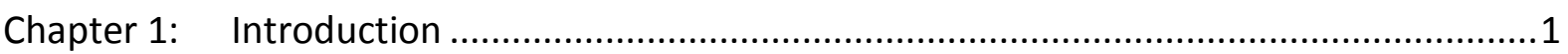

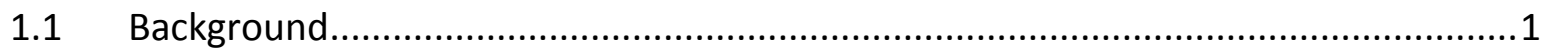

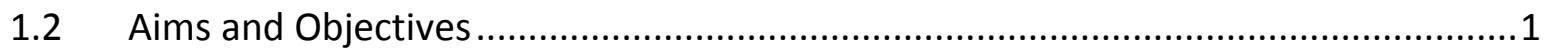

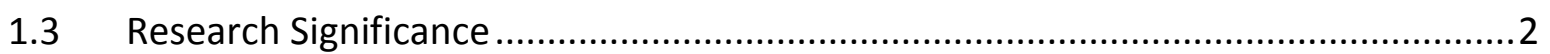

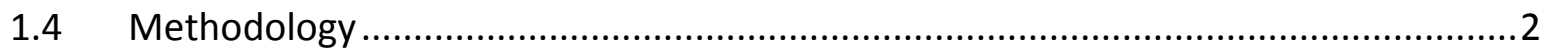

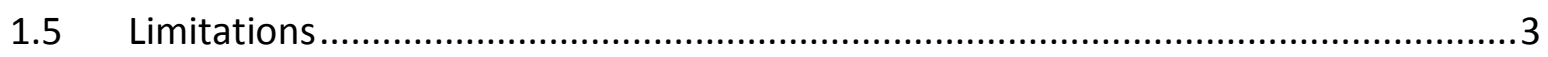

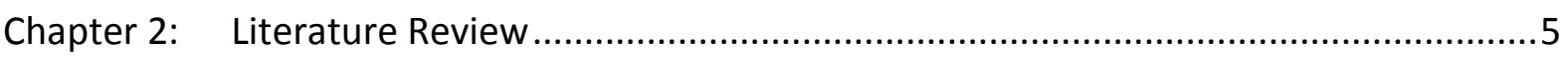

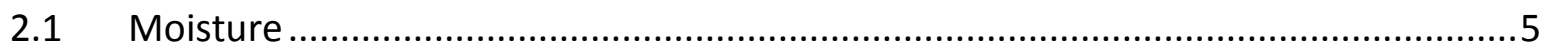

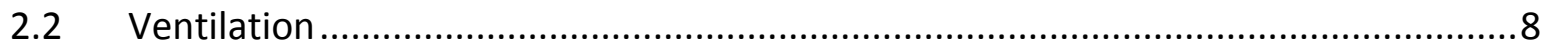

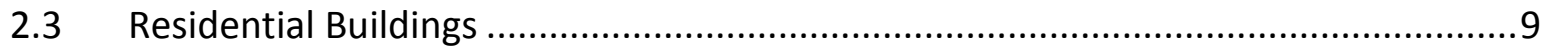

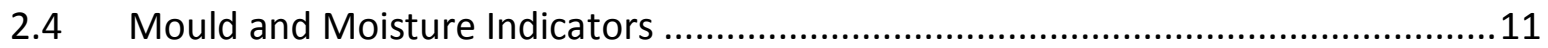

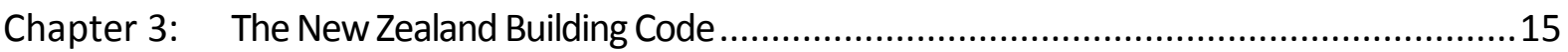

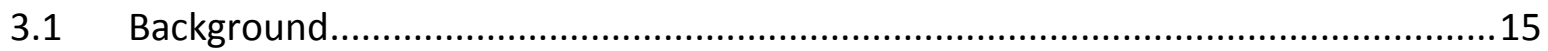

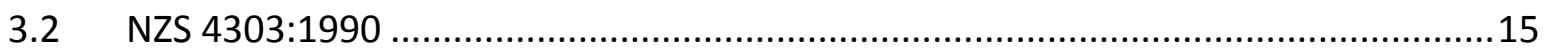

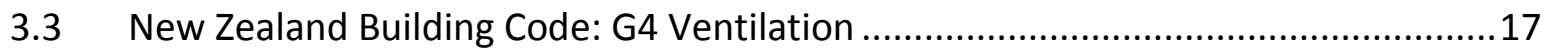

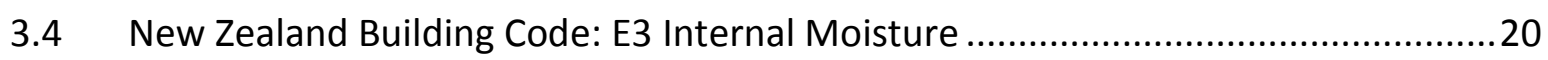




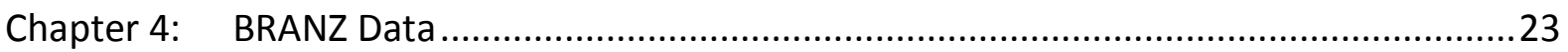

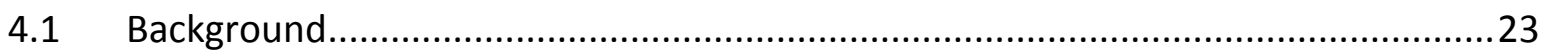

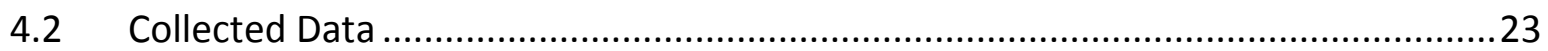

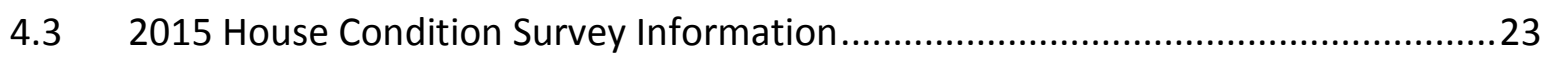

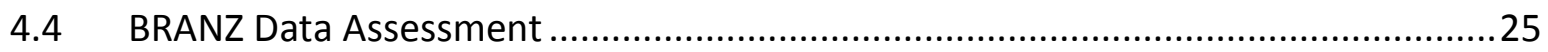

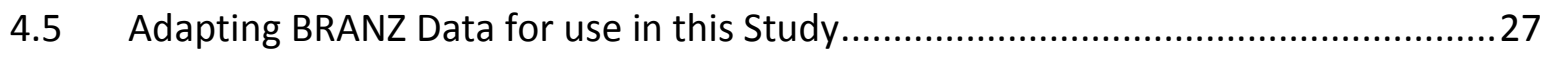

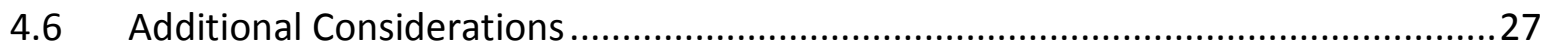

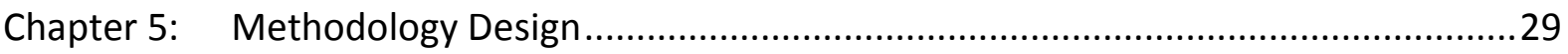

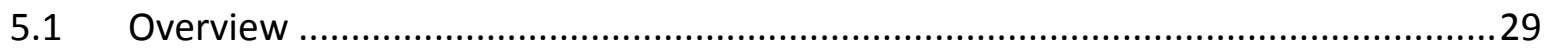

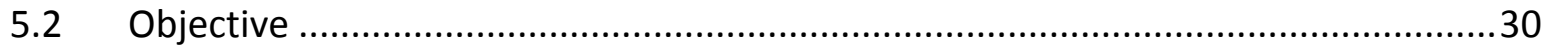

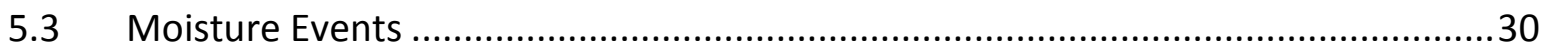

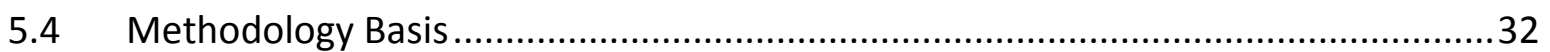

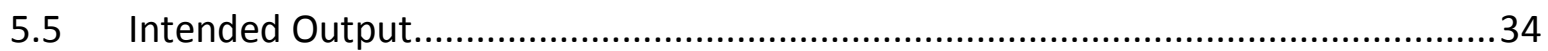

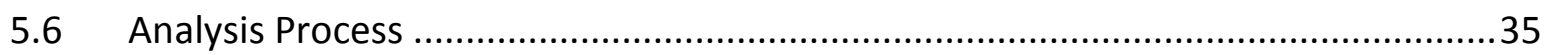

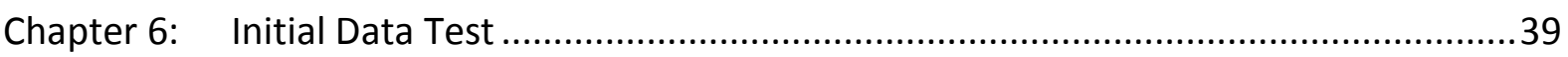

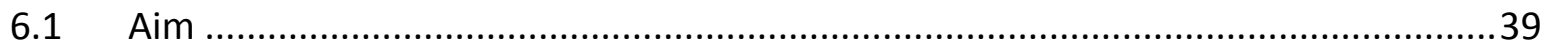

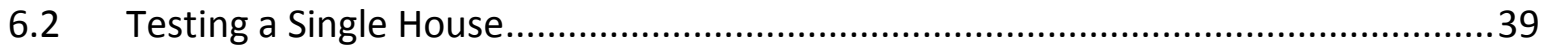

6.3 Objective 1: Testing Required Moisture Event Level ............................................40

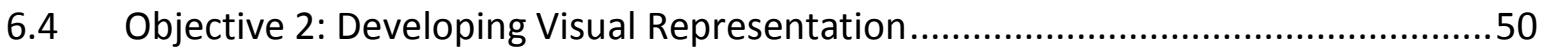

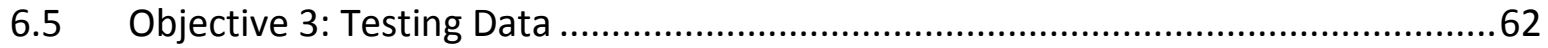

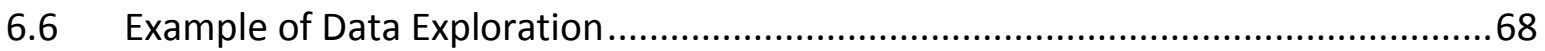

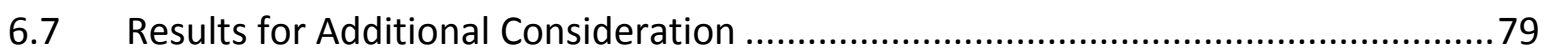

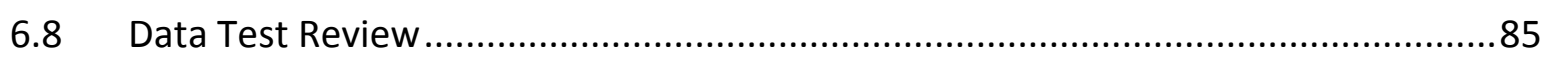

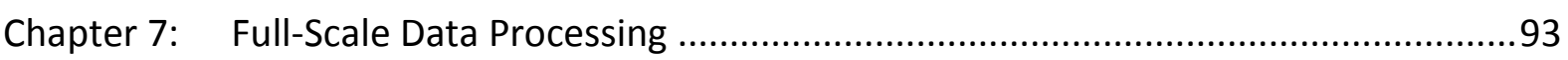

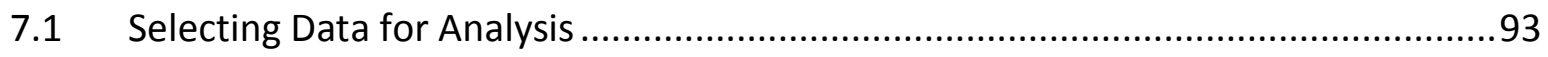

7.2 Data Available for Selected Houses ...................................................................95 


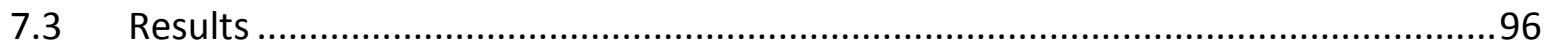

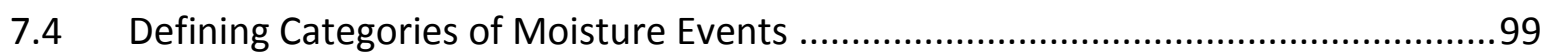

7.5 Filtering Moisture Events for Typical Days ...................................................100

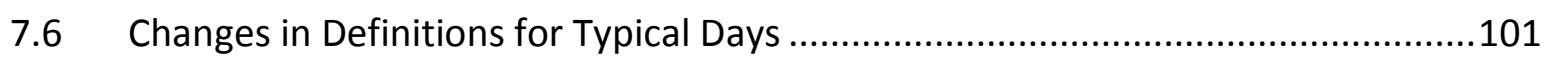

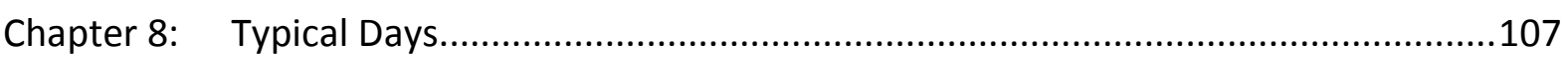

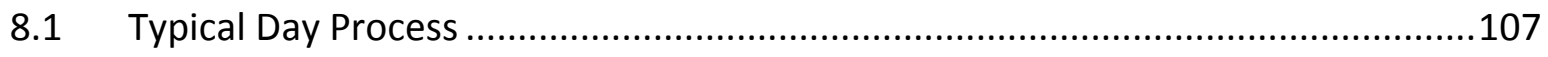

8.2 Example of Categorising Moisture Events: DN1 …...........................................108

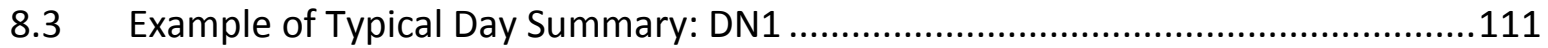

8.4 Example of Typical Day Summary: 2 Bathrooms ............................................113

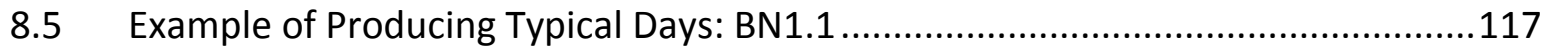

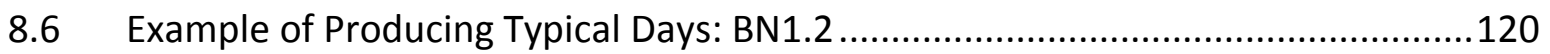

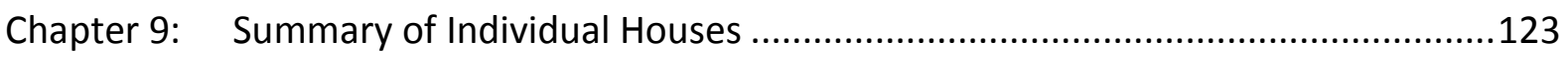

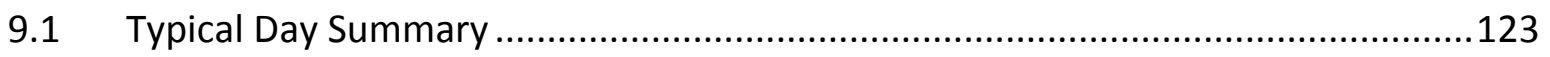

9.2 Mean and Median Absolute Humidity Results ................................................123

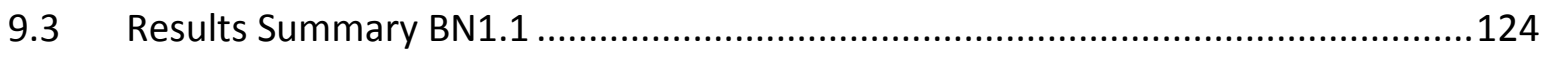

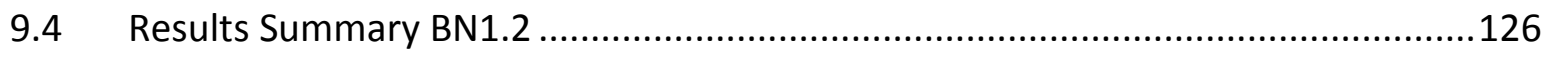

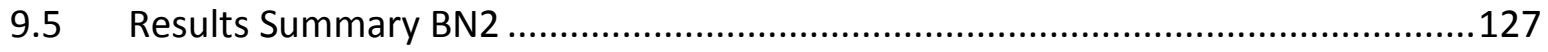

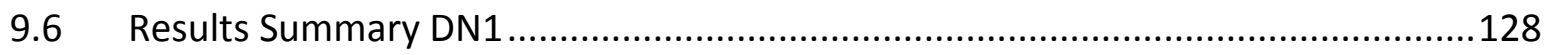

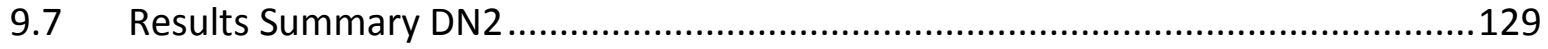

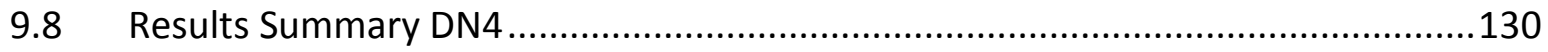

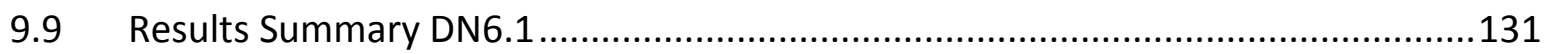

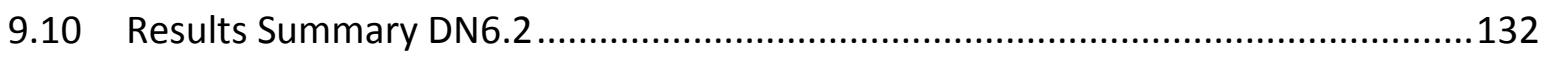

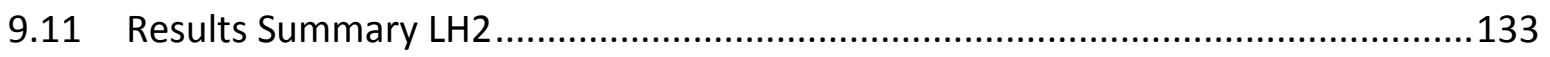

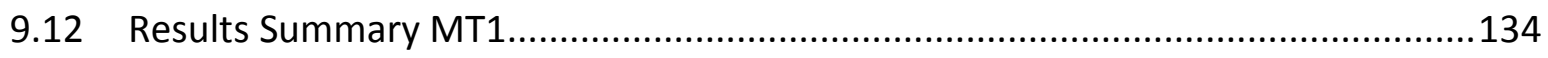

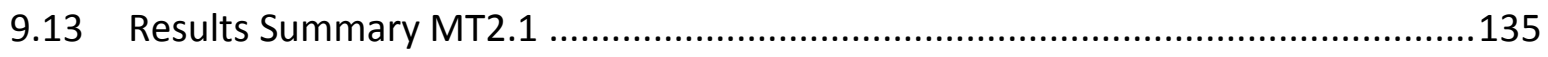

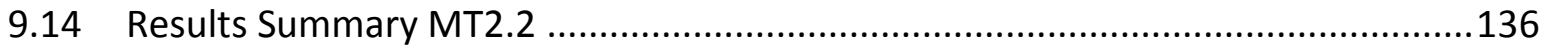




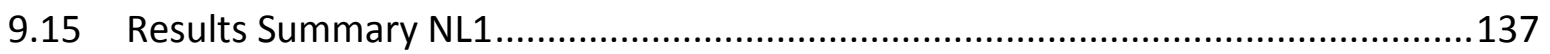

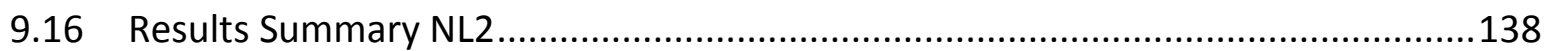

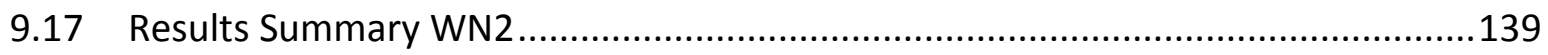

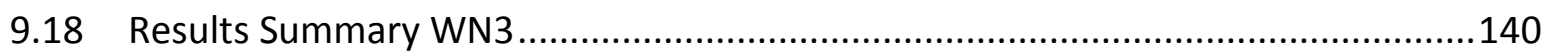

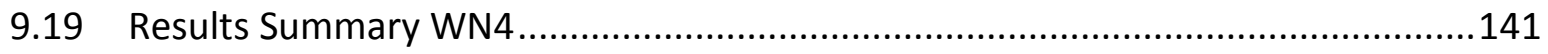

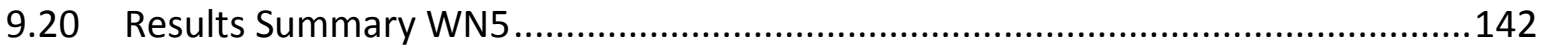

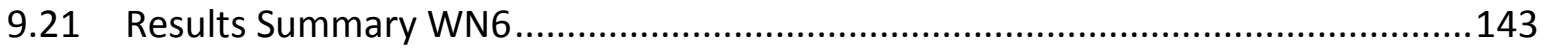

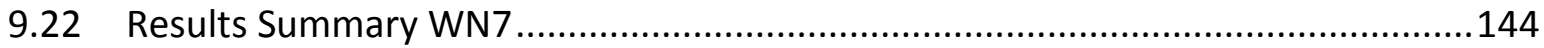

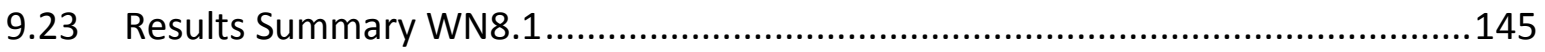

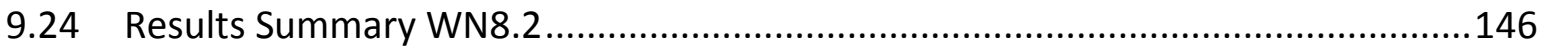

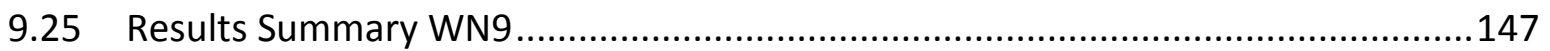

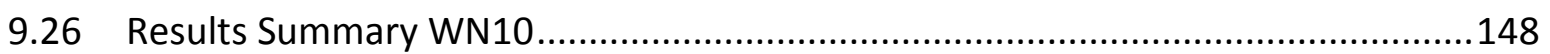

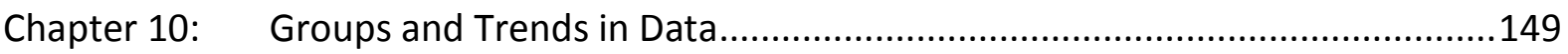

10.1 Mean and Median Episodes for all Bathrooms .....................................................149

10.2 Comparing Results to the Household Energy End-use Project ............................154

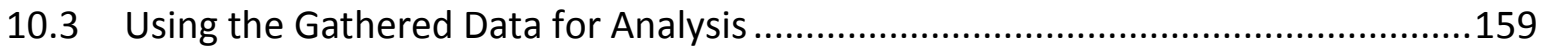

10.4 Taking Starting Levels of Absolute Humidity into Account ...................................161

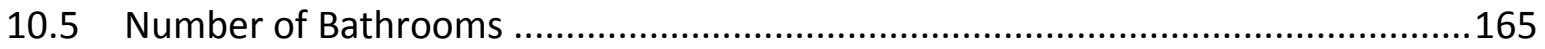

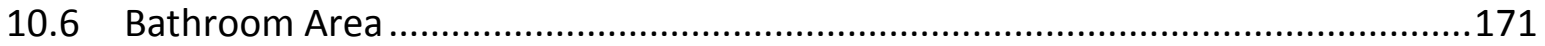

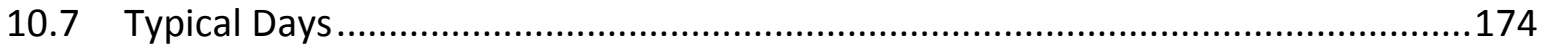

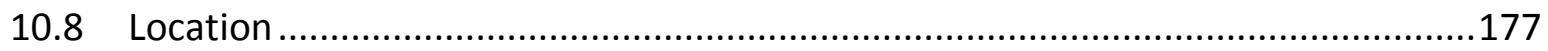

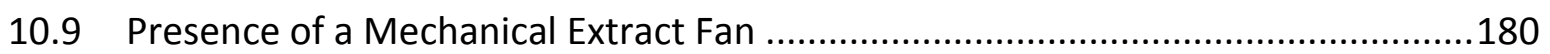

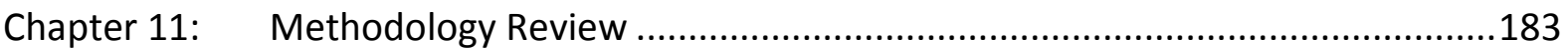

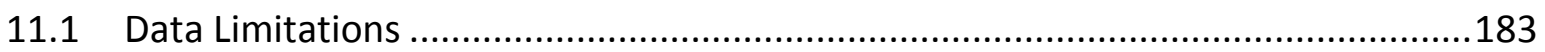

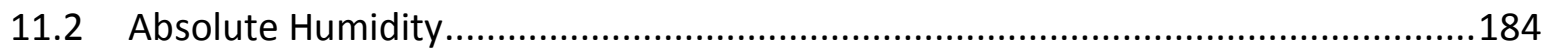

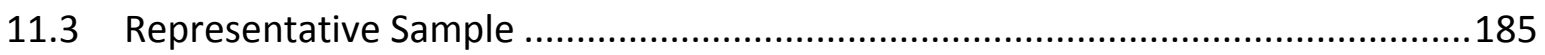




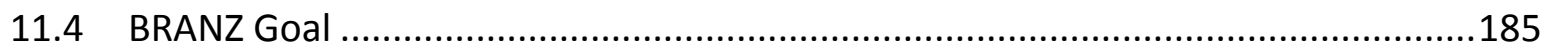

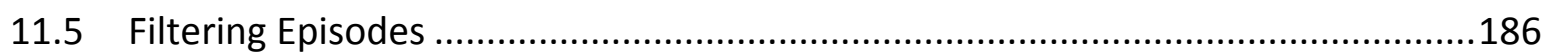

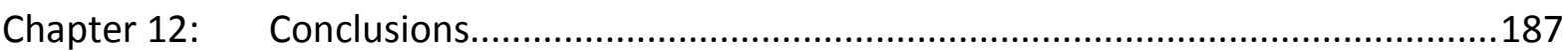

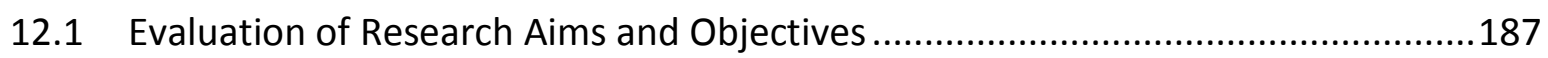

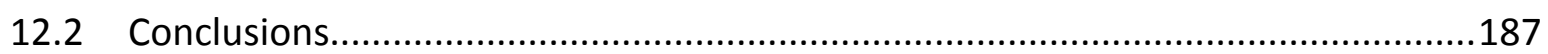

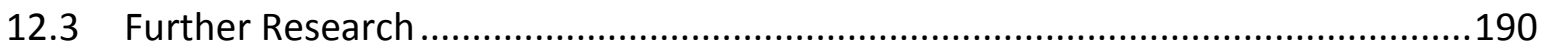

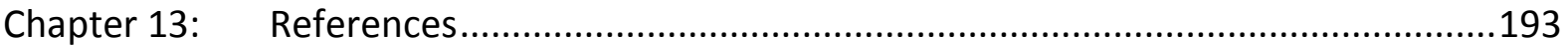

\subsection{List of Figures}

Figure 1 Trickle Vents vs Open Window (McDowall, 2017) .................................................

Figure 2 Presence of Openable Windows and Mechanical Ventilation in Bathrooms (White \&

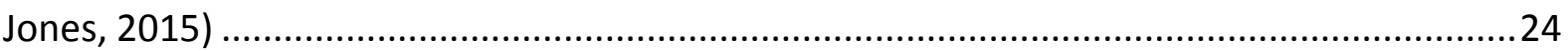

Figure 3 Houses with heating in the Main Bathrooms (White \& Jones, 2015) ......................24

Figure 4 Presence of Heating and Ventilation in Bathrooms (White \& Jones, 2015).............25

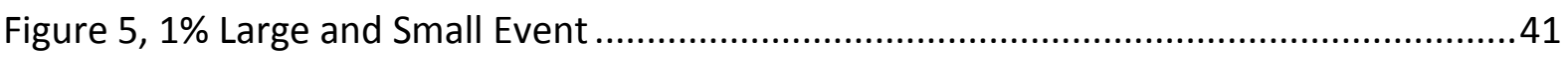

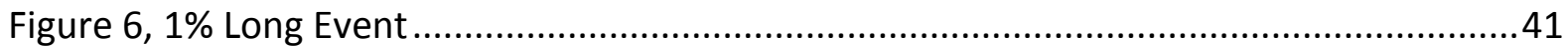

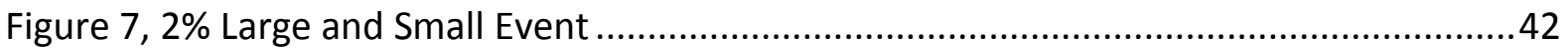

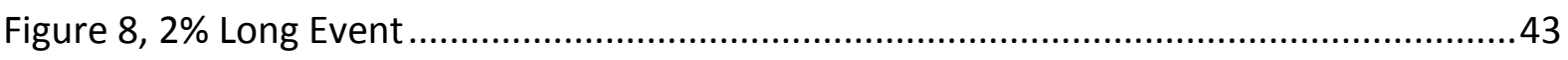

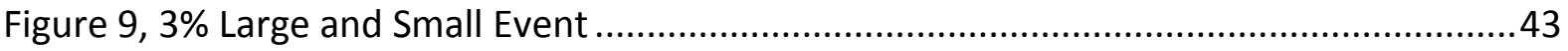

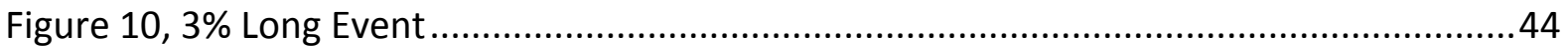

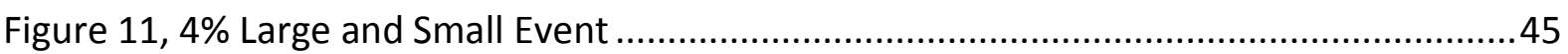

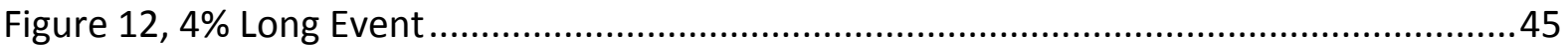

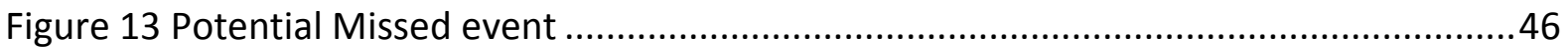

Figure 14 DN4, 'Potential Moisture Event' Introduced at 2\%, Large and Small Event ...........47

Figure 15 DN4, 'Potential Moisture Event' Introduced at 2\%, Large and Small Event ............47

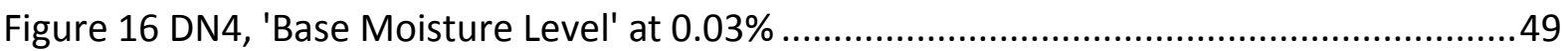

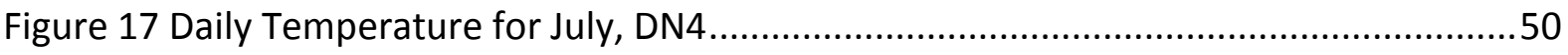

ix

Moisture in New Zealand Bathrooms 


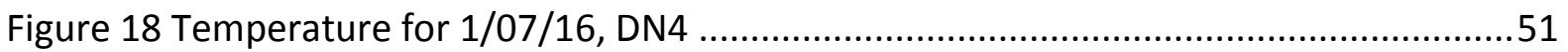

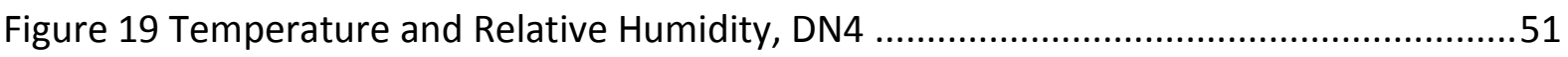

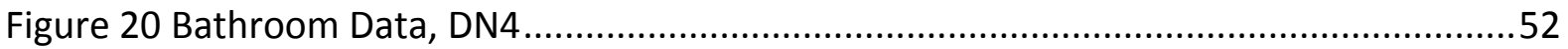

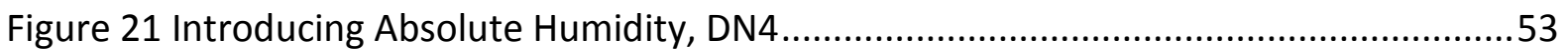

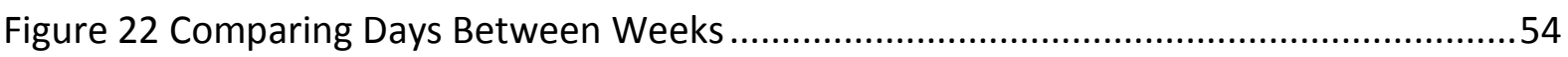

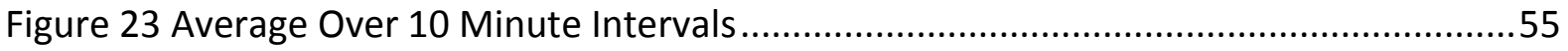

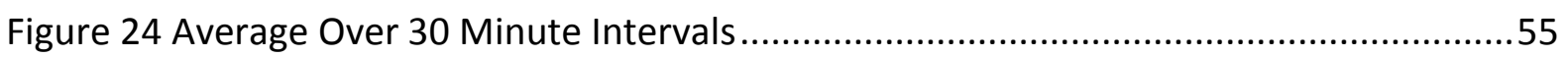

Figure 25 Identifying Moisture Events and Switching Between Days ..................................57

Figure 26 Incorporating Previous Improvements ..............................................................58

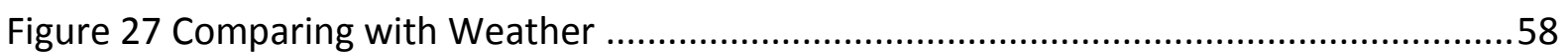

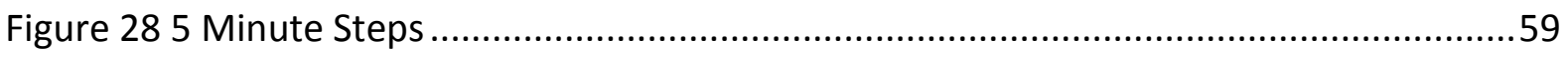

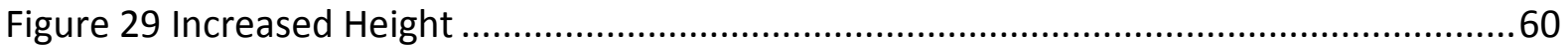

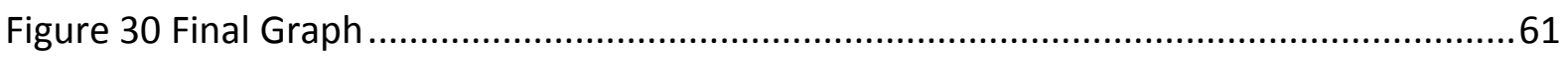

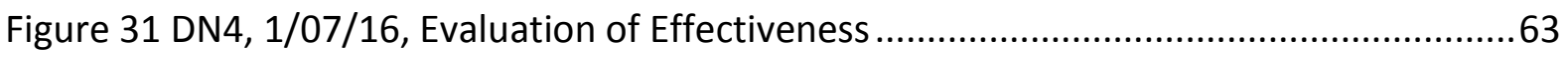

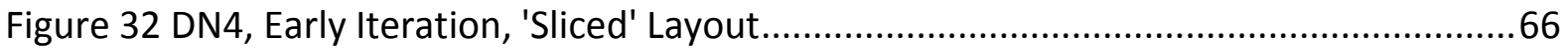

Figure 33 DN4, 01/07/16 - Weather (Temperature and Absolute Humidity) .......................69

Figure 34 DN4, 14/07/16 - Weather (Temperature and Absolute Humidity) .......................70

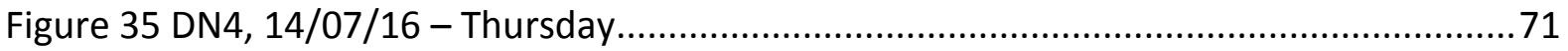

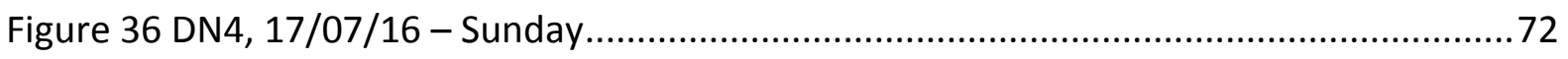

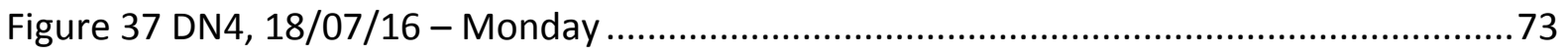

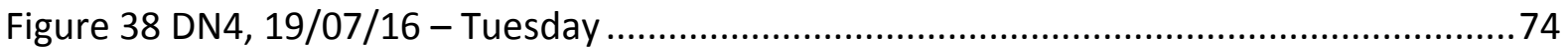

Figure 39 DN4, 20/07/16 - Wednesday ....................................................................... 75

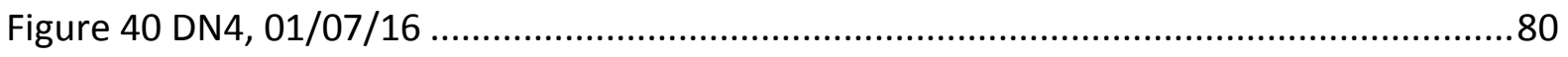

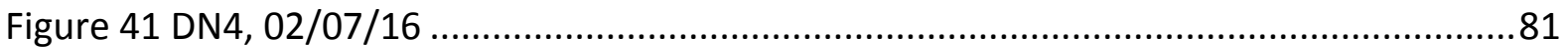

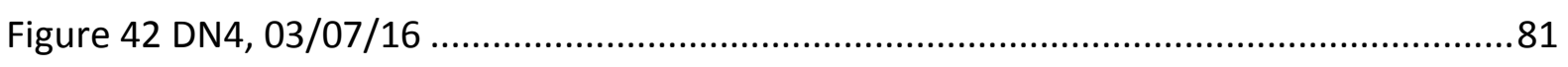

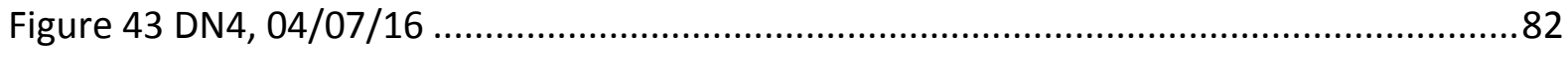

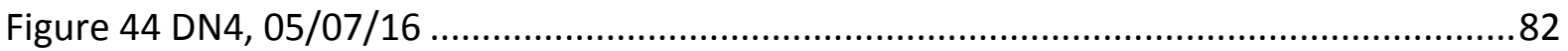

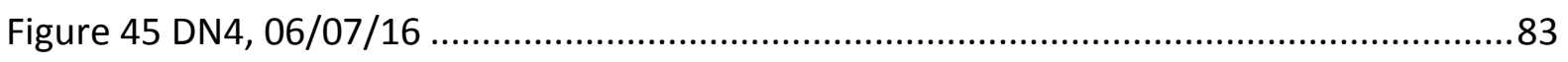

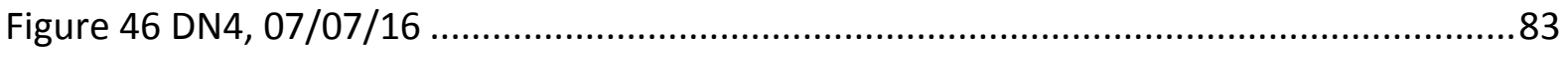

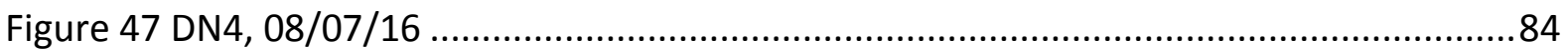

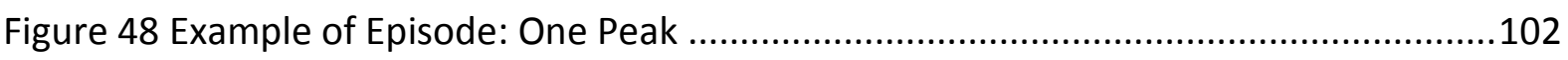




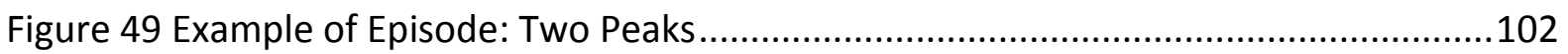

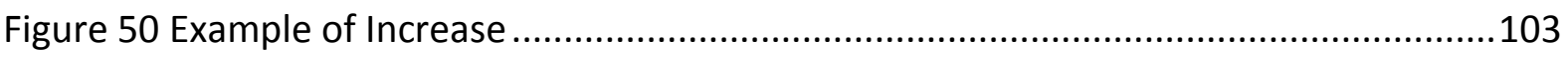

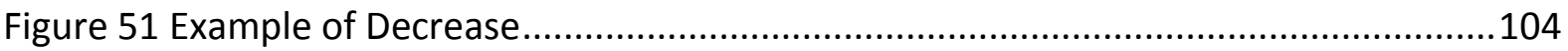

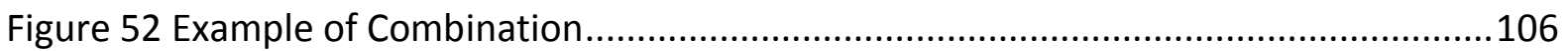

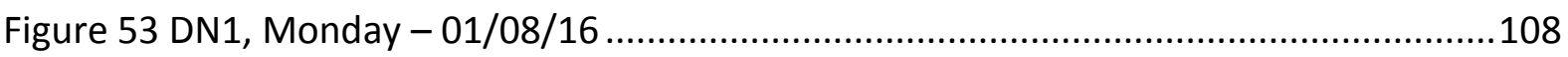

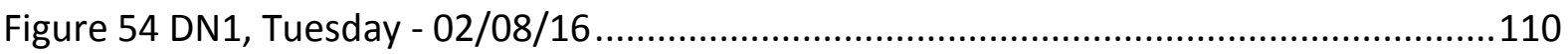

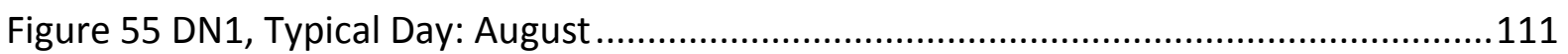

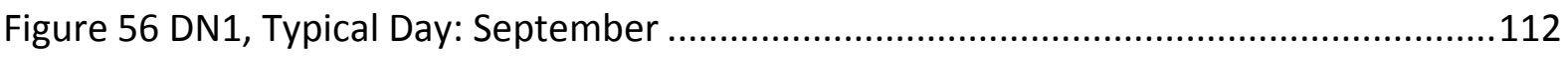

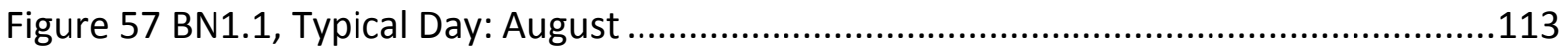

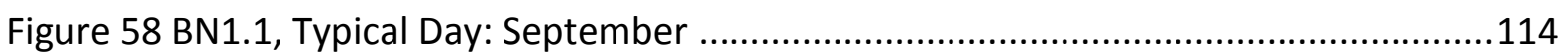

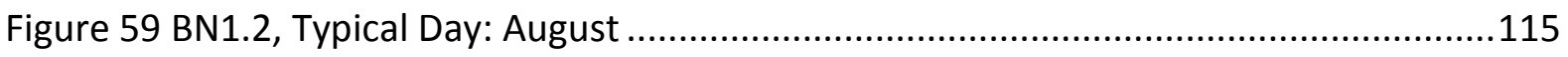

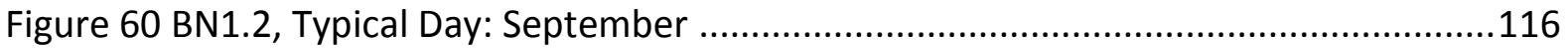

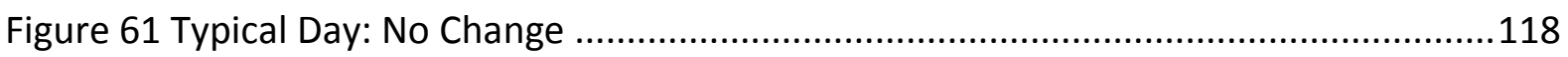

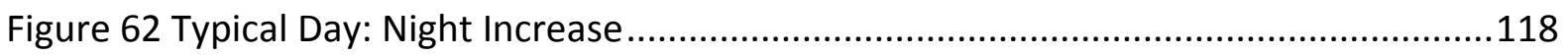

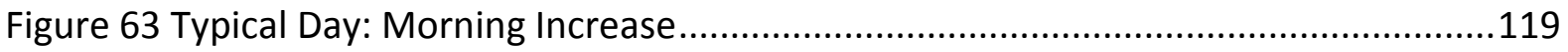

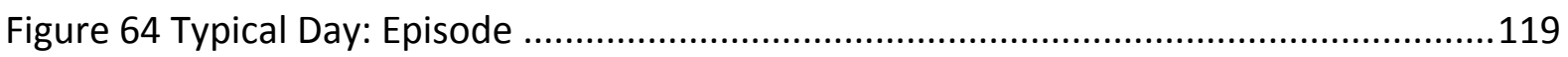

Figure 65 Average Absolute Humidity for Episodes in Bathroom BN1.1 ...........................124

Figure 66 Average Absolute Humidity for Episodes in Bathroom BN1.2 ….......................126

Figure 67 Average Absolute Humidity for Episodes in the Bathroom of House BN2 ...........127

Figure 68 Average Absolute Humidity for Episodes in the Bathroom of House DN1 ..........128

Figure 69 Average Absolute Humidity for Episodes in the Bathroom of House DN2 ..........129

Figure 70 Average Absolute Humidity for Episodes in the Bathroom of House DN4 ….......130

Figure 71 Average Absolute Humidity for Episodes in Bathroom DN6.1 ...........................131

Figure 72 Average Absolute Humidity for Episodes in Bathroom DN6.2 ..........................132

Figure 73 Average Absolute Humidity for Episodes in the Bathroom of House LH2 ...........133

Figure 74 Average Absolute Humidity for Episodes in the Bathroom of House MT1 ...........134

Figure 75 Average Absolute Humidity for Episodes in Bathroom MT2.1...........................135

Figure 76 Average Absolute Humidity for Episodes in Bathroom MT2.2 ...........................136

Figure 77 Average Absolute Humidity for Episodes in the Bathroom of House NL1 ...........137

Figure 78 Average Absolute Humidity for Episodes in the Bathroom of House NL2 ...........138

Figure 79 Average Absolute Humidity for Episodes in the Bathroom of House WN2 .........139

Figure 80 Average Absolute Humidity for Episodes in the Bathroom of House WN3 ..........140

Moisture in New Zealand Bathrooms 
Figure 81 Average Absolute Humidity for Episodes in the Bathroom of House WN4 141

Figure 82 Average Absolute Humidity for Episodes in the Bathroom of House WN5 .142

Figure 83 Average Absolute Humidity for Episodes in the Bathroom of House WN6 143

Figure 84 Average Absolute Humidity for Episodes in the Bathroom of House WN7 144

Figure 85 Average Absolute Humidity for Episodes in Bathroom WN8.1 145

Figure 86 Average Absolute Humidity for Episodes in Bathroom WN8.2 . 146

Figure 87 Average Absolute Humidity for Episodes in the Bathroom of House WN9 147

Figure 88 Average Absolute Humidity for Episodes in the Bathroom of House WN10 ........148

Figure 89 Mean Absolute Humidity at Start, Peak, and End of Episodes .............................150

Figure 90 Mean and Median Length and Absolute Humidity. 151

Figure 91 Mean and Median Length and Absolute Humidity .153

Figure 92 Mean and Median Length of Episodes from Start to Peak .158

Figure 93 Median Absolute Humidity at each Point of Episode compared to Mean Time ...161 Figure 94 Bathrooms Corrected for Start Absolute Humidity compared to Median Time ...162 Figure 95 Bathrooms Corrected for Start Absolute Humidity, Sorted by Peak Absolute Humidity. 163

Figure 96 Bathrooms Corrected for Start Absolute Humidity, Sorted by Start Absolute Humidity. 164

Figure 97 Changes in Absolute Humidity Compared to Start, Houses with One Bathroom .165 Figure 98 Changes in Absolute Humidity Compared to Start, Houses with Two Bathrooms 166 Figure 99 House BN1 Bathroom Comparison 167

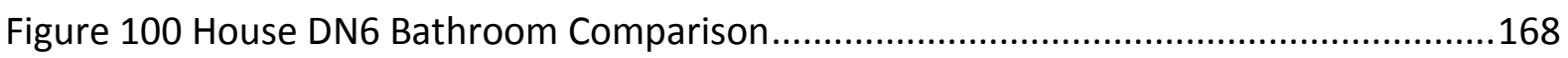

Figure 101 House MT2 Bathroom Comparison .............................................................. 169

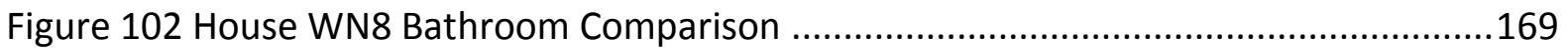

Figure 103 Changes in Absolute Humidity Compared to Start vs Floor Area .......................171

Figure 104 Changes in Absolute Humidity Compared to Start, $3 m^{2}-5 m^{2} \ldots \ldots \ldots \ldots \ldots \ldots \ldots . . . . . . . . . . .171$

Figure 105 Changes in Absolute Humidity Compared to Start, $5 m^{2}-7 m^{2} \ldots \ldots \ldots \ldots \ldots \ldots \ldots \ldots . . . . . . . . . . .172$

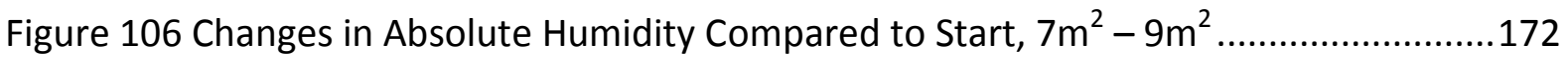

Figure 107 Bathrooms Corrected for Start Absolute Humidity compared to Typical Days with no Moisture Events. 174 
Figure 108 Bathrooms Corrected for Start Absolute Humidity compared to Total Number of Filtered Episodes

Figure 109 Bathrooms Corrected for Start Absolute Humidity compared to Estimated Showers/baths per day

Figure 110 Bathrooms Corrected for Start Absolute Humidity compared to Mean Time:

Blenheim

Figure 111 Bathrooms Corrected for Start Absolute Humidity compared to Mean Time:

Dunedin

Figure 112 Bathrooms Corrected for Start Absolute Humidity compared to Mean Time:

Wellington Region.

Figure 113 Bathrooms Corrected for Start Absolute Humidity compared to Mean Time:

Motueka

Figure 114 Bathrooms Corrected for Start Absolute Humidity compared to Mean Time:

Nelson

Figure 115 Changes in Absolute Humidity Compared to Start, Bathrooms with and Extract Fan

Figure 116 Changes in Absolute Humidity Compared to Start, Bathrooms without an Extract Fan. .180

\subsection{List of Tables}

Table 1: Mould and Moisture Indicators Used by Inspectors During Home Walkthrough Evaluations (Mahooti-Brooks et al., 2004)

Table 2: Association Between Mould/Moisture Indicators in the Basement and bathroom and Physical Measurements (Mahooti-Brooks et al., 2004)

Table 3: Association between Mould/Moisture Indicators and Measures of Airborne Fungi in

Bathroom (Mahooti-Brooks et al., 2004)

Table 4, Outdoor Requirements for Ventilation of Residential Facilities (NZS4303:1990) .....16

Table 5: Recorded Data.....

Table 6 Sample of Measurements from Start of Moisture Events .......................................76

Table 7 Sample of Measurements from Peak of Moisture Events ........................................76

Table 8 Sample of Measurements from End of Moisture Events. 
Table 9 Measurements of a Single Moisture Event............................................................ 78

Table 10 Duration of Each Stage of a Single Moisture Event ................................................ 79

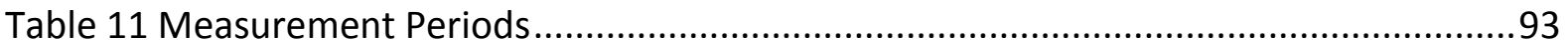

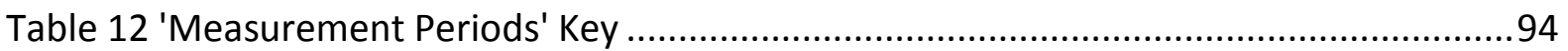

Table 13 Available Data for Selected Houses ....................................................................96

Table 14 'Available Data for Selected Houses' Key.............................................................96

Table 15 DN1, Example of Mean and Median Moisture Event Lengths.................................98

Table 16 DN1, Example of Mean and Median Absolute Humidity at Each Point of Moisture

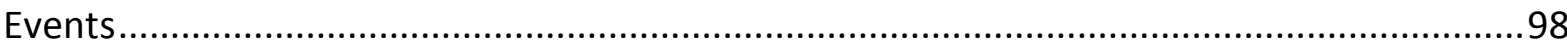

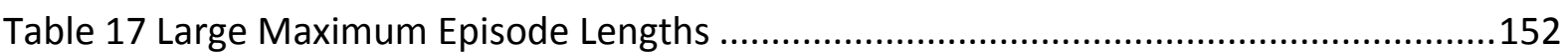

Table 18 Estimated Shower/Bath Use per Day Based on Episodes Filtered for Typical Days

\subsection{Definitions}

These definitions are taken from (Standards New Zealand, 1990) and (Ministry of Business, Innovation \& Employment, 2014)

\begin{tabular}{|l|l|}
\hline Absolute Humidity & $\begin{array}{l}\text { The water content in the air. Measures the mass of water vapour } \\
\text { divided by the mass of dry air in a volume of air. Does not take } \\
\text { temperature into account. }\end{array}$ \\
\hline Absorption & $\begin{array}{l}\text { The process of one substance entering into the inner structure of } \\
\text { another. }\end{array}$ \\
\hline Acceptable indoor air quality & $\begin{array}{l}\text { Air in which there are no known contaminants at harmful } \\
\text { concentrations as determined by cognizant authorities and with } \\
\text { which a substantial majority (80\% or more) of the people exposed do } \\
\text { not express dissatisfaction. }\end{array}$ \\
\hline Adequate & Achieves the objectives of the building code. \\
\hline Air-cleaning system & $\begin{array}{l}\text { A device or combination of devices applied to reduce the } \\
\text { concentration of airborne contaminants, such as microorganisms, } \\
\text { dusts. Fumes, respirable particles, other particulate matter, gases, } \\
\text { and/or vapours in air. }\end{array}$ \\
\hline Air conditioning & $\begin{array}{l}\text { The process of treating air to meet the requirements of a conditioned } \\
\text { space by controlling its temperature, humidity, cleanliness, and } \\
\text { distribution. }\end{array}$ \\
\hline Air, outdoor & $\begin{array}{l}\text { Air taken from the external atmosphere and, therefore, not } \\
\text { previously circulated through the system. Typically comprising by } \\
\text { volume 20.94\% oxygen, 0.03\% carbon dioxide, and 79.03\% nitrogen } \\
\text { and other inert gases. It also contains a variable quantity of moisture. }\end{array}$ \\
\hline Amenity & $\begin{array}{l}\text { An attribute of a building which contributes to the health, physical } \\
\text { independence, and well-being of the building's users, but which is not }\end{array}$ \\
\hline
\end{tabular}




\begin{tabular}{|c|c|}
\hline & associated with disease or a specific illness. \\
\hline Concentration & $\begin{array}{l}\text { The quantity of one constituent dispersed in a defined amount of } \\
\text { another. }\end{array}$ \\
\hline Conditioned space & $\begin{array}{l}\text { That part of a building that is heated or cooled, or both, for the } \\
\text { comfort of occupants. }\end{array}$ \\
\hline Contaminant & $\begin{array}{l}\text { An unwanted airborne constituent that may reduce acceptability of } \\
\text { the air. }\end{array}$ \\
\hline Dust & $\begin{array}{l}\text { An air suspension of particles (aerosol) of any solid material, usually } \\
\text { with particle size less than } 100 \text { micrometres. }\end{array}$ \\
\hline Fumes & $\begin{array}{l}\text { Airborne particles, usually less than } 1 \text { micrometre in size, formed by } \\
\text { condensation of vapours, sublimation, distillation, calcination, or } \\
\text { chemical reaction. }\end{array}$ \\
\hline Gas & $\begin{array}{l}\text { A state of matter in which substances exist in the form of non- } \\
\text { aggregated molecules, and which, within acceptable limits of } \\
\text { accuracy, satisfies the ideal gas laws; usually superheated vapour. }\end{array}$ \\
\hline Habitable space & $\begin{array}{l}\text { A space used for activities normally associated with domestic living, } \\
\text { but excludes any bathroom, laundry, water closet, pantry, walk-in } \\
\text { wardrobe, corridor, hallway, lobby, clothes-drying room, or other } \\
\text { space of a specialised nature occupied neither frequently nor for } \\
\text { extended periods. }\end{array}$ \\
\hline Household unit & $\begin{array}{l}\text { A building or group of buildings, or part of a building or group of } \\
\text { buildings, that is used, or intended to be used, only or mainly for } \\
\text { residential purposes, and that is occupied, or intended to be } \\
\text { occupied, exclusively as the home or residence of not more than one } \\
\text { household, but that does not include a hostel, boarding house, or } \\
\text { other specialised accommodation. }\end{array}$ \\
\hline HVAC & An abbreviation for heating, ventilation, and air-conditioning. \\
\hline HVAC system & $\begin{array}{l}\text { A mechanical, electrical, or other system for modifying air } \\
\text { temperature, modifying air humidity, providing ventilation, or doing } \\
\text { all or any of those things, in a space within the building. }\end{array}$ \\
\hline Infiltration & $\begin{array}{l}\text { Air leakage inward through cracks and interstices and through } \\
\text { ceilings, floors, and walls of a space or building. }\end{array}$ \\
\hline Microorganism & $\begin{array}{l}\text { A microscopic organism, especially a bacterium, fungus, or a } \\
\text { protozoan. }\end{array}$ \\
\hline Natural ventilation & $\begin{array}{l}\text { The movement of outdoor air into a space through intentionally } \\
\text { provided openings, such as windows and doors, or through non- } \\
\text { powered ventilators or infiltration. }\end{array}$ \\
\hline Occupied zone & $\begin{array}{l}\text { The region within an occupied space between planes } 75 \mathrm{~mm} \text { and } \\
1800 \mathrm{~mm} \text { above the floor and more than } 600 \mathrm{~mm} \text { from the walls or } \\
\text { fixed air-conditioning equipment. }\end{array}$ \\
\hline Odour & $\begin{array}{l}\text { A quality of gases, liquids, or particles that stimulates the olfactory } \\
\text { organ. }\end{array}$ \\
\hline Particulate matter & $\begin{array}{l}\text { A state of matter in which solid or liquid substances exist in the form } \\
\text { of aggregated molecules or particles. Airborne particulate matter is } \\
\text { typically in the size range of } 0.01 \text { to } 100 \text { micrometres. }\end{array}$ \\
\hline Smoke & $\begin{array}{l}\text { The airborne solid and liquid particles and gases that evolve when a } \\
\text { material undergoes pyrolysis or combustion. Note: chemical smoke is } \\
\text { excluded from this definition. }\end{array}$ \\
\hline Vapour & $\begin{array}{l}\text { A substance in gas form, particularly one near equilibrium with its } \\
\text { condensed phase, which does not obey the ideal gas laws; in general, } \\
\text { any gas below its critical temperature. }\end{array}$ \\
\hline Ventilation & $\begin{array}{l}\text { The process of supplying and removing air by natural or mechanical } \\
\text { means to and from any space. Such air may or may not be }\end{array}$ \\
\hline
\end{tabular}


Alister Stubbe

conditioned. 
Moisture in New Zealand Bathrooms 



\section{Chapter 1: Introduction}

\subsection{Background}

Moisture is a serious issue for many homes around the country. It is constantly produced from a variety of sources and is often not handled effectively, resulting in condensation, mould, and mildew. These problems are widespread in New Zealand and can cause significant damage to room surfaces.

This can have serious consequences for both the occupants and the building itself. Damp homes create dangerous living conditions, making rooms difficult to warm. Cold, damp houses pose serious health risks, especially for young children and the elderly who spend most of their time at home. 1600 more people die in winter than any other season in New Zealand. These winter mortality rates are among the highest of any developed country that measures this statistic. An Otago study attributed many of these deaths to cold, damp living conditions.

Building practices have improved over the last few decades and New Zealand are building homes to be as energy efficient as possible, reducing the amount of heat lost to air leakage. Together these mean houses are becoming more airtight. While this helps keep heat inside, it also prevents moisture from escaping. Trapping this wet air inside raises the absolute humidity, making it harder to heat the indoor environment. Better understanding which factors play the greatest role in influencing moisture sources may identify current practices that could be improved, helping to prevent this moisture from entering the indoor environment in the first place. To do this, periods where moisture is being introduced must be identified and better understood.

\subsection{Aims and Objectives}

This study aims to identify and analyse periods of rapid change in moisture levels in a set of bathrooms. This will include both the types of change common in each house, the frequency of these changes, and the amount of moisture they introduce or remove. Completing this would require seven key objectives: 
- Create a process for identifying periods where moisture is being rapidly introduced or removed from the indoor environment (moisture events).

- Test this process on data gathered on houses measured by the Building Research Association of New Zealand (BRANZ) as part of the House Condition Survey (HCS).

- Identify the time large moisture events occur each day.

- Identify recurring patterns in the times these large moisture events occur for each day.

- Measure the average length of periods of rapid moisture change in each house.

- Calculate the average amount of moisture present in the indoor environment at each point during periods of rapid moisture change in each house.

- Compare findings from each house, identifying common trends.

\subsection{Research Significance}

Completing these objectives will help to identify the periods where the indoor environment, specifically the bathroom, is most at risk from high moisture levels. This includes information on how frequently these occur, how long they last, and how large they were. These results could be compared to self-reported use of moisture sources such as showers, providing information on how many periods of rapid change are caused by occupant activity.

\subsection{Methodology}

The methodology had five steps designed to complete the objectives of this study:

- define types of moisture events;

- identify typical days;

- calculate average moisture episodes;

- compare average moisture episodes to typical days; and

- identify trends and groups between different houses.

Data from one house was first tested and used to inform the rest of the study. 32 bathrooms from 28 houses were then processed, identifying moisture events. This was 
Moisture in New Zealand Bathrooms

compared with the available information from each house, analysing which factors had the greatest impact.

\subsection{Limitations}

The study was limited by both the availability of relevant data as well as the amount of time available. With information available on changes in temperature and humidity rather than the room itself, there was no simple method of checking whether the results were an accurate reflection of the indoor environment. The small sample size of available houses means that further analysis is required to generalise these results.

The study was further limited by the location of the houses available for study. The analysed houses were from Blenheim, Dunedin, Lower Hutt, Motueka, Nelson, and Wellington. This did not represent the entire country. The results of this study may not apply to areas in New Zealand with a different local climate, such as Auckland. 
Alister Stubbe 


\section{Chapter 2: Literature Review}

\subsection{Moisture}

This section outlines the importance of considering the control and impact of moisture. It examines the conditions that allow moisture damage to occur and how buildings can degrade to this state. Solutions to this are discussed, identifying the key components of moisture control.

Moisture can cause a variety of issues. These include the degradation of building materials, the growth of mould and other biological forms, and structural failure. For a moisturerelated problem to occur, there are four conditions to be met: a moisture source, a route or means for moisture to travel, a driving force to cause moisture movement, and for the materials involved to be susceptible to moisture damage (Straube, 2002).

It is practically impossible to remove all moisture sources, to create buildings with no imperfections, or to remove all forces driving moisture. It is uneconomical to use only materials that are not susceptible to moisture damage. Instead, a balance between wetting and drying can be maintained to prevent moisture from accumulating. Most moisture control strategies focus on reducing wetting such as by increasing airtightness. However, over time faults are likely to accumulate and allow greater amounts of water to enter the building (Straube, 2002).

Possible steps for managing indoor moisture include limiting sources of water, effectively heating the indoor environment, regularly opening windows, and installing an appropriate ventilation system. There are a wide variety of ventilation systems, ranging from passive vents to mechanical extraction. Windows are a common form of ventilation in residential dwellings (McNeil, 2016). Ventilation, insulation, and heating are the key components of ensuring a healthy indoor environment (McNeil, Plagmann, McDowall, \& Bassett, 2015). If left untreated, moisture issues can cause a variety of problems. These include health issues, building materials decay, and the growth of mould and fungi. As New Zealand has

5

Literature Review 
shifted away from strip flooring and timber window frames, buildings have become more airtight. This means buildings rely far more on ventilation to remove moisture. Houses such as this depend on occupants understanding how to operate their building to prevent mould growth problems (Su, 2006). Clause G4 of the New Zealand Building Code (NZBC)may need to be updated, as it does not allow for occupant behaviour (McNeil, 2016).

$33 \%$ of the BRANZ site visit database consists of issues relating to water. Around half of these were condensation related. Most of these relate to walls, but roof leakage problems were also an issue. Indoor moisture problems do not necessarily indicate rainwater is entering wall cavities. The indoor environment will often show little evidence of a water leak inside the wall. Condensation will occur on the coldest part of the envelope, potentially the windows or an uninsulated ceiling. This condensation results in a moist environment, where dust mites thrive. Carpets have also been shown to support high concentrations of dust mites, making high relative humidity readings deep in carpets especially concerning. There is a strong link between dust mites and asthma in New Zealand (Bassett, 2011).

New Zealanders spend 55\% of their times inside their homes, meaning the issues caused by moisture are likely to affect occupants directly. With $45 \%$ of New Zealand homes reporting mould, this is a serious issue (Popenhagen, 2012). New Zealand has the second highest rate of asthma in the world and high rates of respiratory related illnesses, with one in four children having asthma, as well as one of the highest rates of winter related deaths (Leardini \& Van Raamsdonk, 2010). Respiratory diseases resulted in 2663 deaths in New Zealand during 2013 and cost the country $\$ 6.16$ Billion (Barnard \& Zhang, 2016). The risk of asthma increases with the severity of moisture damage and presence of visible mould in a dwelling's main living quarters. Results carried out in a Finnish study suggest moisture and mould damage in these areas are associated with the development of asthma in early childhood (Pekkanen et al., 2007).

The Control of Substance Hazardous to Health (COSHH) hierarchy of measures indicates the most effective means of controlling moisture (Bassett, 2011).

1. Eliminate the source of pollutant (e.g., dry clothes outside, remove any unflued gas heaters).

2. Substitute another material for source. 
3. Enclose/isolate the source (e.g., pressure cooker, subfloor ground cover).

4. Local extract and partial enclosure (e.g., bathroom extract fan, range hood).

5. Dilution ventilation (e.g., passive vents, whole building ventilation).

6. Personal protection.

Actions such as removing an unflued gas heater or turning on a bathroom extract fan have a large impact on how effectively moisture is controlled. This highlights the importance of the occupant's role in moisture management. Their actions have the potential to improve (or sabotage) each of the other steps. Failing to follow these steps, such as by not opening a window to extract moisture from the space, directly affects the indoor environment.

Opening windows at

certain times would solve

many of the moisture problems in New Zealand houses. A few behavioural changes could make the difference between a damp home and a dry home. BRANZ carried out a case study demonstrating window opening performance, as stipulated in Building Code Clause G4 (Figure 1). A north-facing test house was conditioned with heating and moisture to $25^{\circ} \mathrm{C}$ and relative humidity to $70 \%$.
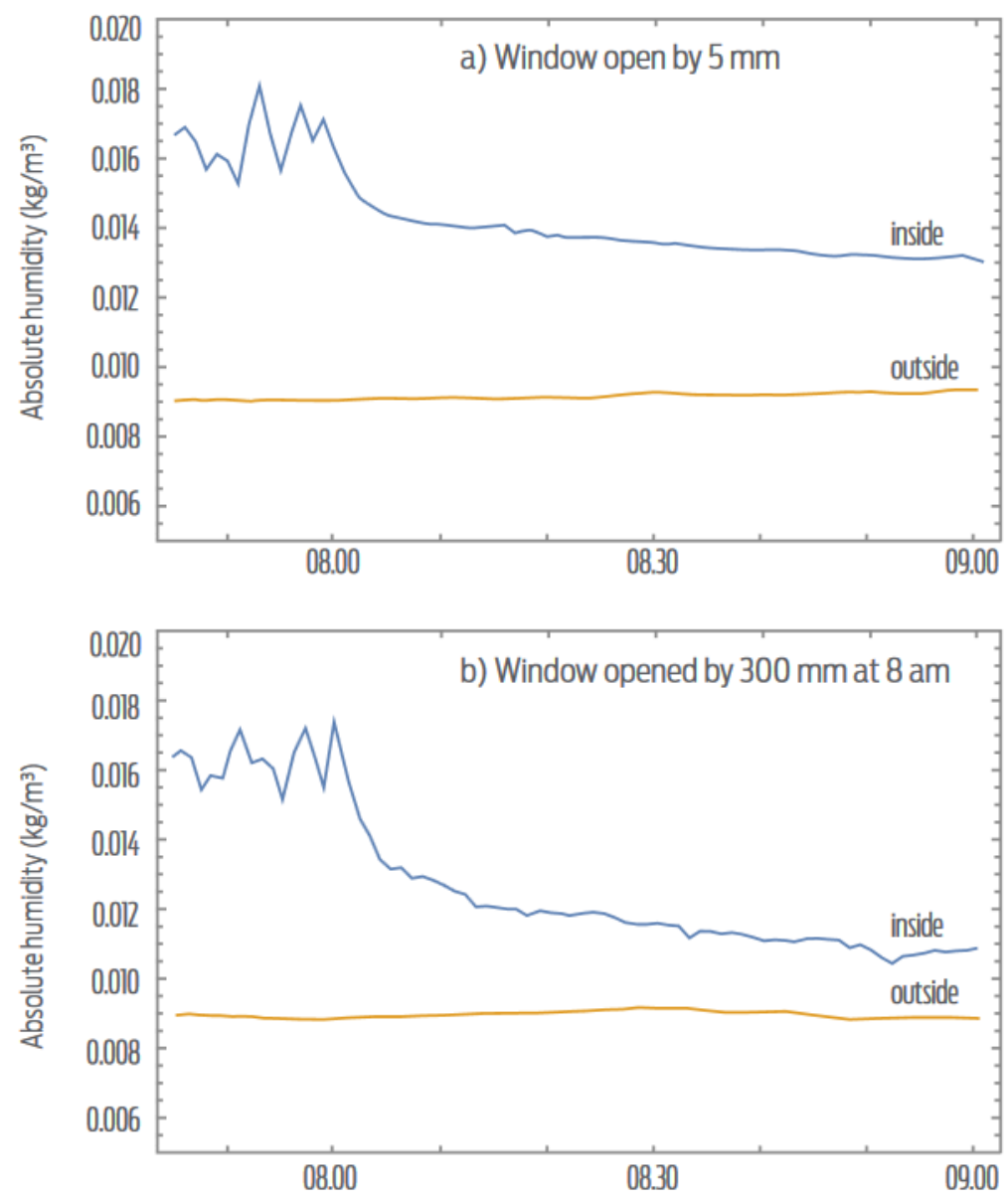

Figure 1 Trickle Vents vs Open Window (McDowall, 2017)

The windows were opened by $5 \mathrm{~mm}$ (similar to a trickle vent) and the doors were closed. Despite the room being heated to $25^{\circ} \mathrm{C}$, condensation formed on the ceiling, walls, and windows. During experiments, environmental conditioning was turned off at 8.00am and a $1030 \times 735 \mathrm{~mm}$ window was opened by various amounts up to $300 \mathrm{~mm}$. Within 10 minutes of 
the window being opened by the full $300 \mathrm{~mm}$, the airborne moisture content had reduced by $14 \%$. By contrast, trickle vents only reduced airborne moisture content by $4.5 \%$ in 10 minutes and would take hours to remove the same amount of moisture. After this initially rapid drying, the humidity decreased at a slower rate, as the condensation on the ceiling, walls, and windows evaporates slowly. Ideally, the room would be heated first, evaporating the moisture and allowing it to be more easily removed. (McDowall, 2017).

\subsection{Ventilation}

This section considers the impact of ventilation in maintaining a healthy indoor environment. This includes both the role ventilation plays in removing moisture from the home and how occupants influence the effectiveness of these systems.

Moisture and ventilation are closely related, as the number of air changes per hour (ACH) determine how effectively moisture within a space is removed. This makes air circulation an important consideration when evaluating the impact of moisture.

As previously described, New Zealand homes are becoming more airtight (McNeil \& Rupp, 2017). A survey of airtightness and ventilation rates in New Zealand houses found houses built since 1995 have an average infiltration of 6.7 ACH compared to 8.5 ACH from houses built in the previous decade. Most houses meet the NZBC Clause G4 minimum requirements for ventilation, but perfluorocarbon tracer measurements showed most houses struggle to reach recommended levels of ventilation because windows were not open often enough. The recommended level of ventilation is between 0.35 and $0.5 \mathrm{ACH}$ during the winter (McNeil, Quaglia, Plagmann, \& Overton, 2011).

In recent years it has become more common for occupants to install additional supply-only ventilation to control moisture. Most houses with these systems had more than enough ventilation. However, there were several cases where the ventilation system had been turned off (McNeil et al., 2011).

The impact of moisture in homes is an issue around the world. A Danish study which investigated the association between environmental risk factors and asthma/allergies among young children used $\mathrm{CO}_{2}$ concentration in bedrooms to measure ventilation rates. It 
found around $57 \%$ of bedrooms had ventilation rates lower than the required Danish minimum of $0.5 \mathrm{~h}^{-1}$ (Bekö, Lund, Nors, Toftum, \& Clausen, 2010).

How occupants use ventilation is just as important. Owners of new houses in Ontario, Canada, were surveyed about ventilation systems and how they use them. The data collected shows many ventilation systems are either used incorrectly or not at all (Canada Mortgage and Housing Corporation, 2004).

Factors influencing how occupants control ventilation systems such as windows can be grouped into five main categories:

- Physical environment (temperature, humidity, air velocity, noise, illumination, odour, etc.);

- Contextual (building condition, heating system type, orientation, etc.);

- Psychological (occupants tend to satisfy their needs concerning comfort and safety. They have certain expectations of environmental quality);

- Physiological (age, gender, health, clothing, activity level, food/beverage intake);

- Social (the interaction between occupants, e.g., household composition).

The occupant reacts consciously or unconsciously to stimulus to improve/restore/maintain comfort conditions (Fabi, Andersen, Corgnati, \& Olesen, 2012). An empirical study carried out in a naturally ventilated high-rise office building in the UK indicates window operation is strongly correlated with outdoor air temperature, the season, the time of day, and occupancy patterns (Zhang \& Barrett, 2012). A Danish study of residential buildings found similar results, identifying outdoor temperature and air quality (identified by $\mathrm{CO}_{2}$ concentration) as the two most important variable influencing the probability of windows being opened or closed (Andersen, Fabi, Toftum, Corgnati, \& Olesen, 2013). An analysis of occupant behaviour literature suggests occupants will attempt to restore their comfort in the easiest way possible and are influenced by contextual factors (O’Brien \& Gunay, 2014).

\subsection{Residential Buildings}

This section considers the consequences of occupant control discussed in the ventilation section. It compares the impact incorrect operation of ventilation systems may have, the 
opportunity for occupants of residential buildings to misuse or misunderstand ventilation systems, and the risk moisture generation in residential buildings poses. The effectiveness of ventilation is reliant on occupant control. This makes residential buildings especially susceptible to incorrect operation. While occupants of office buildings often have limited control over their environment, occupants in residential buildings are more likely to have direct control over the systems in use.

Incorrect system operation can be most easily identified in the areas of a home which most consistently experience issues with internal moisture, showing the first signs of moisture damage. The internal moisture load depends on the rate of moisture generation from various sources, the net removal rate due to ventilation or air-conditioning, and the moisture-holding capacity of hygroscopic building materials and furniture (Yik, Sat, \& Niu, 2004). The bathroom is an ideal location to gather data on moisture when considering these variables. The main moisture sources are similar for most bathrooms, the rate of moisture generation is high enough that any regular changes are more likely to be measurable, ventilation and/or air-conditioning are present in almost all cases, and there are unlikely to be many unprotected hygroscopic materials or furniture present. This means there are fewer unmeasured variables influencing bathrooms, making it easier to compare results from different houses.

Day-to-day variation cannot be entirely attributed to differences in use. The amount of moisture generated by the same household activity can vary widely. For example, the amount of moisture bathing generates in a four-member household could range from 200 to $2400 \mathrm{~g} /$ day (Yik et al., 2004). This could be due to differences in water temperature, whether occupants took a tub-bath or shower-bath, or the duration of bathing. This makes it difficult to conclusively state whether increases and decreases in humidity were due to how control systems were operated or how household activities were carried out without data being gathered on a wide range of variables measured over a period long enough to identify trends in occupant behaviour.

In a Hong Kong study on moisture generation in households, occupants were asked about their habits for moisture generating activities. Most people indicated they would take shower-baths once every day using hot water, typically between $9.00 \mathrm{pm}$ and $11.00 \mathrm{pm}$. The 
average duration was 18 minutes. Nobody indicated they took tub-baths daily. The majority of household members would turn on the extract fan instead of opening a window during a bath, but only one-third of people would keep the bathroom fan running for some time after the bath (Yik et al., 2004).

The Household Energy End-use Project (HEEP) monitored fuel types and the services they provide over a national random sample of $400 \mathrm{New}$ Zealand homes. The number of selfreported showers/baths per day per house was $2.5 \pm 0.1$ while the average number showers per person per day was $0.9 \pm 0.5$ (Camilleri, Burrough, Pollard, Saville-Smith, \& Fraser, 2010). The self-reported average shower duration was $9.5 \pm 0.2$ minutes, although shower durations varied widely. Assuming an average flow rate of 8.4 litres per minute, the average warm water consumption was 200 litres per day for showers. On average, occupants reported taking 0.4 baths per day per household, or 0.14 baths per person per day. Assuming the average bath uses 150 litres of warm water, the average warm water consumption is 60 litres per day for baths.

\subsection{Mould and Moisture Indicators}

This section considers how a potentially damp or otherwise unhealthy indoor environment can be identified. It looks at how the presence of certain factors or measurements of an indoor space may indicate the presence of mould or excessive moisture.

There is a wide range of potential indicators of a damp environment. These include visible mould damage, active water leakage, building materials detected to be moist, relative humidity exceeding $80 \%$ in drill-hole measurements, or a material sample showing active microbial growth (Annila et al., 2017). These indicators focus on the extent of moisture and mould damage rather than the impact they have on air quality.

These indicators are important for evaluating damage to buildings as well as for tools evaluating potential repairs (Annila et al., 2017). A list of these indicators may be used by inspectors to evaluate buildings for the presence of mould in different rooms (MahootiBrooks et al., 2004).

\begin{tabular}{|c|l|l|}
\hline Room & \multicolumn{1}{|c|}{ Indicator } & \multicolumn{1}{c|}{ Definition } \\
\hline Bathroom & Absent exhaust fan & $\begin{array}{l}\text { The bathroom fan is not present or is not functional. It is not } \\
\text { externally exhausted to the outside. }\end{array}$ \\
\hline
\end{tabular}

11

Literature Review 


\begin{tabular}{|c|c|c|}
\hline & Visible moisture damage & $\begin{array}{l}\text { The presence of water damage. For example, the presence of } \\
\text { water stains on the ceiling, walls, or around the windows. } \\
\text { Bubbling paint on the windowsill or other areas. }\end{array}$ \\
\hline & $\begin{array}{l}\text { Visible mould in the } \\
\text { shower }\end{array}$ & $\begin{array}{l}\text { The visible presence of fungal colonies of any colour on } \\
\text { surfaces, including tiles, walls, and ceilings in the tub/shower } \\
\text { enclosure. }\end{array}$ \\
\hline & $\begin{array}{l}\text { Visible mould in other } \\
\text { areas }\end{array}$ & $\begin{array}{l}\text { The visible presence of fungal colonies of any colour on } \\
\text { surfaces, including tiles, sinks, walls, ceilings, toilet, windows, } \\
\text { or floors in other areas of the bathroom excluding the } \\
\text { tub/shower enclosure. }\end{array}$ \\
\hline \multirow[t]{5}{*}{ Basement } & Water Sources & $\begin{array}{l}\text { Any information or signs related to leaks, defective drainage, } \\
\text { water accidents, uncontrolled water activity, or water } \\
\text { intrusion events in the basement at any period during the } \\
\text { occupant's time of living in the dwelling and/or during the } \\
\text { life of the dwelling. }\end{array}$ \\
\hline & Wall efflorescence & $\begin{array}{l}\text { The presence of 'crystals' or white markings on the walls in } \\
\text { the basement. }\end{array}$ \\
\hline & Spalling & $\begin{array}{l}\text { The breaking off or bursting out of a surface layer of concrete } \\
\text { or brickwork, usually caused by freezing of intruded water. } \\
\text { This is often present as holes, divots, or surface defects. }\end{array}$ \\
\hline & Musty odour & A damp, musty smell is noticed when entering the room. \\
\hline & Visible mould & $\begin{array}{l}\text { The visible presence of fungal colonies of any colour on } \\
\text { surfaces, walls, ceilings, windows, or floors, most often small } \\
\text { colonies on surfaces without. }\end{array}$ \\
\hline \multirow[t]{3}{*}{ Bedroom } & Visible moisture damage & $\begin{array}{l}\text { The presence of water damage. The presence of water stains } \\
\text { on the ceiling, walls, or around the windows. Bubbling paint } \\
\text { on the windowsill or other areas. }\end{array}$ \\
\hline & Visible mould & $\begin{array}{l}\text { The visible presence of fungal colonies of any colour on } \\
\text { surfaces, walls, ceilings, windows, or floors. }\end{array}$ \\
\hline & Wall-to-wall carpet & Carpeting is present from wall to wall in the room. \\
\hline
\end{tabular}

Table 1: Mould and Moisture Indicators Used by Inspectors During Home Walkthrough Evaluations (Mahooti-Brooks et al., 2004)

Table 1 provides an example from an American study of the indicators used to identify rooms at potential risk of mould and moisture damage during an inspection. Carpeting was included because of its potential to act as a reservoir of microbial growth. Results from an evaluation of 58 houses using these indicators were compared with physical measurements (Mahooti-Brooks et al., 2004).

\begin{tabular}{|c|l|l|c|}
\hline Room & \multicolumn{1}{|c|}{ Indicator } & \multicolumn{1}{|c|}{ No. Houses } & Relative Humidity (\%) Mean \\
\hline Basement & \multirow{2}{*}{ Musty Odour } & Present: 38 & 55.8 \\
\cline { 3 - 4 } & & Absent: 20 & 46.6 \\
\cline { 2 - 4 } & \multirow{2}{*}{ Efflorescence } & Present: 24 & 58.7 \\
\cline { 3 - 4 } & & Absent: 34 & 48.4 \\
\cline { 2 - 4 } & \multirow{2}{*}{ Visible Mould } & Present: 15 & 59.1 \\
\cline { 3 - 4 } & & Absent: 43 & 50.4 \\
\cline { 2 - 4 } & \multirow{2}{*}{ Water Sources } & Present: 37 & 57.3 \\
\cline { 3 - 4 } & & Absent: 20 & 45.4 \\
\hline Bathroom & Exhaust Fan & Present: 35 & 43.7 \\
\cline { 3 - 4 } & & Absent: 28 & 49.4 \\
\hline
\end{tabular}


Table 2: Association Between Mould/Moisture Indicators in the Basement and bathroom and Physical Measurements (Mahooti-Brooks et al., 2004)

\begin{tabular}{|c|c|c|c|c|c|c|}
\hline \multirow[t]{2}{*}{ Room } & \multirow[t]{2}{*}{ Indicator } & \multirow[t]{2}{*}{ No. Houses } & \multicolumn{2}{|c|}{$\begin{array}{c}\text { Total Airborne Fungi } \\
\text { (Colony Forming Units } / \mathrm{m}^{3} \text { ) }\end{array}$} & \multicolumn{2}{|c|}{$\begin{array}{l}\text { Moisture Indicator Fungi } \\
\left.\text { (Colony Forming Units } / \mathrm{m}^{3}\right)\end{array}$} \\
\hline & & & $\begin{array}{l}\text { Indoor } \\
\text { (Mean) }\end{array}$ & $\begin{array}{l}\text { Outdoor } \\
\text { (Mean) }\end{array}$ & $\begin{array}{l}\text { Indoor } \\
\text { (Mean) }\end{array}$ & $\begin{array}{l}\text { Outdoor } \\
\text { (Mean) }\end{array}$ \\
\hline \multirow[t]{10}{*}{ Bathroom } & \multirow{2}{*}{$\begin{array}{l}\text { Exhaust } \\
\text { fan }\end{array}$} & Present: 35 & 536 & 752 & 503 & 667 \\
\hline & & Absent: 28 & 1029 & 1019 & 935 & 103 \\
\hline & \multirow{2}{*}{$\begin{array}{l}\text { Visible } \\
\text { Mould in } \\
\text { Shower }\end{array}$} & Present: 33 & 901 & 1147 & 853 & 1029 \\
\hline & & Absent: 28 & 564 & 590 & 505 & 511 \\
\hline & \multirow{2}{*}{$\begin{array}{l}\text { Visible } \\
\text { Mould in } \\
\text { other than } \\
\text { shower }\end{array}$} & Present: 12 & 861 & 1764 & 805 & 1613 \\
\hline & & Absent: 48 & 711 & 698 & 654 & 610 \\
\hline & \multirow{2}{*}{$\begin{array}{l}\text { Any visible } \\
\text { mould }\end{array}$} & Present: 35 & 819 & 1086 & 778 & 978 \\
\hline & & Absent: 26 & 619 & 603 & 549 & 519 \\
\hline & \multirow{2}{*}{$\begin{array}{l}\text { Visible } \\
\text { moisture } \\
\text { damage }\end{array}$} & Present: 18 & 697 & 568 & 641 & 484 \\
\hline & & Absent: 43 & 740 & 999 & 683 & 895 \\
\hline
\end{tabular}

Table 3: Association between Mould/Moisture Indicators and Measures of Airborne Fungi in Bathroom (Mahooti-Brooks et al., 2004)

Homes with mould and moisture indicators in the basement were found to have a statistically significant higher relative humidity than homes without these indicators (Table 2) (Mahooti-Brooks et al., 2004). Likewise, bathroom mould and moisture indicators indicate that where these are present, or where an exhaust fan is absent, the total amount of airborne fungi increases compared to outside (Table 3). This demonstrates the connection between mould/moisture indicators, measurements of relative humidity, and measurements of airborne fungi. 
Alister Stubbe 


\section{Chapter 3: The New Zealand Building Code}

\subsection{Background}

As outlined in Chapter 2: Literature Review, buildings must be designed to deal with moisture sources to maintain a healthy indoor environment. The NZBC describes how buildings must be designed and built to meet these requirements.

The NZBC consists of three general clauses and 38 technical clauses. The sections of direct relevance to maintaining a healthy environment for bathrooms are E3 (Internal Moisture) and G4 (Ventilation).

It aims to demonstrate the acceptable solutions for buildings, how these can be verified, and potential alternative solutions. It does not take into account how occupants may use the building, including extract fans and windows. As a result, a building that theoretically meets the Building Code could still potentially suffer from moisture damage as a consequence of being incorrectly operated by its occupants.

\subsection{NZS 4303:1990}

NZS4303:1990 specifies the minimum ventilation rates and indoor air quality acceptable to human occupants, and is intended to avoid adverse health effects(Standards New Zealand, 1990). The goal of this standard is to achieve acceptable air quality while also minimising energy consumption. These considerations often conflict, meaning a balance needs to be struck between them.

\subsubsection{Systems and Equipment}

Ventilation may be mechanical or natural. When mechanical ventilation is used, provision for airflow measurement should be included. When natural ventilation and infiltration are relied upon, sufficient ventilation shall be demonstrable. Mechanical ventilation must be provided in situations where natural ventilation and infiltration are insufficient. Ventilation systems should be designed and installed so ventilation air is provided throughout the occupied zone. These systems should not conflict with standards for thermal environmental 
conditions for human occupancy. Provisions should be made to maintain acceptable indoor air quality throughout the occupied zone when the supply of air is reduced.

Ventilating systems should be designed to prevent exhaust contaminants, condensation, and growth of microorganisms from re-entering the building (or becoming a nuisance to the occupants of neighbouring buildings) after being discharged. Ventilating ducts should be constructed and maintained to minimise the opportunity for growth and dissemination of microorganisms throughout the ventilation system. Notably, contaminants from stationary local sources within the indoor environment should be controlled by collection and removal as close to the source as practicable. This is especially relevant when considering moisture control in bathrooms, outlining that ventilation in these spaces should aim to remove moisture as soon as it is introduced to the indoor environment. Other contaminants discussed in NZS4303:1990 include smoke and dust.

Rooms with high humidity can support the growth of pathogenic or allergenic organisms. These include certain species of fungi, associated mycotoxins, and dust mites. This growth is enhanced by the presence of materials with high cellulose, even with low nitrogen content, such as fibreboard, dust lint, skin particles, and dander. Areas of concern include bathrooms and bedrooms. Relative humidity in habitable spaces preferably should be maintained between $30 \%$ and $60 \%$ to minimise the growth of allergenic or pathogenic organisms.

\begin{tabular}{|c|c|c|}
\hline Applications & Outdoor Requirements & Comments \\
\hline Living areas & $\begin{array}{l}0.35 \text { air changes per hour but not } \\
\text { less than } 15 \mathrm{cfm}(7.5 \mathrm{~L} / \mathrm{s}) \text { per person }\end{array}$ & $\begin{array}{l}\text { For calculating the air changes per hour, the volume of the living spaces shall include all } \\
\text { areas within the conditioned space. The ventilation is normally satisfied by infiltration and } \\
\text { natural ventilation. Dwellings with tight enclosures may require supplemental ventilation } \\
\text { supply for fuel-burning appliances, including fireplaces and mechanically exhausted appliances. } \\
\text { Occupant loading shall be based on the number of bedrooms as follows: first bedroom, two } \\
\text { persons; each additional bedroom, one person. Where higher occupant loadings are known, } \\
\text { they shall be used. }\end{array}$ \\
\hline Kitchens $^{\mathrm{b}}$ & $\begin{array}{l}100 \mathrm{cfm}(50 \mathrm{~L} / \mathrm{s}) \text { intermittent or } 25 \\
\mathrm{cfm}(12 \mathrm{~L} / \mathrm{s}) \text { continuous or openable } \\
\text { windows }\end{array}$ & $\begin{array}{l}\text { Installed mechanical exhaust capacity'. Climatic conditions may affect choice of the ventila- } \\
\text { tion system. }\end{array}$ \\
\hline $\begin{array}{l}\text { Baths, } \\
\text { Toilets }\end{array}$ & $\begin{array}{l}50 \mathrm{cfm}(25 \mathrm{~L} / \mathrm{s}) \text { intermittent or } 20 \\
\mathrm{cfm}(10 \mathrm{~L} / \mathrm{s}) \text { continuous or openable } \\
\text { windows }\end{array}$ & Installed mechanical exhaust capacity. \\
\hline $\begin{array}{l}\text { Garages: } \\
\text { Separate for } \\
\text { each dwell- } \\
\text { ing unit }\end{array}$ & $100 \mathrm{cfm}(50 \mathrm{~L} / \mathrm{s})$ per car & Normally satisfied by infiltration or natural ventilation \\
\hline $\begin{array}{l}\text { Common for } \\
\text { several units }\end{array}$ & $1.5 \mathrm{cfm} / \mathrm{ft}^{2}\left(7.5 \mathrm{~L} / \mathrm{s} \cdot \mathrm{m}^{2}\right)$ & See "Enclosed parking garages," Table 2.1 \\
\hline
\end{tabular}

Table 4, Outdoor Requirements for Ventilation of Residential Facilities (NZS4303:1990) 
The requirements for the ventilation of residential buildings are outlined in NZS4303:1990, Table 2.3 (pg. 11) (Table 4). For bathrooms, this includes providing either 25 litres/second (L/s) of intermittent ventilation, $10 \mathrm{~L} / \mathrm{s}$ of continuous ventilation, or openable windows.

\subsubsection{Ventilation Requirements}

NZS4303:1990 states that indoor air quality shall be considered acceptable if the required rates of outdoor air specified in Table 4 are provided for the occupied space(Standards New Zealand, 1990). There are exceptions to this, such as when unusual indoor contaminants are present or anticipated, or for areas in industrial facilities not covered by Table 4 .

The required ventilation rates set out in NZS4303:1990 are listed in litres per second (L/S) per person or litres per second, per square metre. Contamination is normally assumed to be in proportion to the number of people in the indoor environment. In other cases, the contamination is presumed to be chiefly due to other factors and the ventilation rates are based on more appropriate parameters. In bathrooms, the chief consideration for designing ventilation is the number of active moisture sources, especially considering many bathrooms are designed to be used by only one person at a time.

\subsection{New Zealand Building Code: G4 Ventilation}

Clause G4 of the Building Code aims to safeguard people from illness or loss of amenity due to lack of fresh air. It is based on AS/NZS 3666:- Part 1 and 2: 2011, NZS 4303:1990, AS/NZS4740:2000, AS/NZS 5601:- Part 1: 2010, and AS 1668:- Part 2: 2002.

Clause G4 states that spaces within buildings shall be provided with adequate ventilation consistent with their maximum occupancy and their intended use. There are five ventilation performance goals for buildings:

- Spaces within buildings will have the means to ventilate with outdoor air that will provide an adequate number of air changes to maintain air purity.

- Mechanical air-handling systems will be constructed and maintained in a manner that prevents harmful bacteria, pathogens, and allergens from multiplying within them.

- Buildings will have a means of collecting or removing products from the indoor environments in which they are generated, including (bold added):

17

The New Zealand Building Code 
- cooking fumes and odours;

$\bigcirc$ moisture from laundering, utensils washing, bathing, and showering;

○ gaseous by-products and excessive moisture from the commercial or industrial processes;

○ poisonous fumes and gases;

○ airborne particles;

- bacteria, viruses, or other pathogens; and

- products of combustion.

- Contaminated air should be disposed of without creating a nuisance or hazard to people and other property.

- The quantities of air supplied should meet the additional demand of any fixed combustion appliances.

Natural ventilation of occupied spaces must be achieved by providing a net openable area of windows or other openings to the outside of no less than $5 \%$ of the floor area. This requirement does not apply to commercial and industrial building or household units where there is only one external wall with openable windows.

For kitchens, bathrooms, toilets, and laundries that have an external wall, moisture must be ventilated to the outside by natural ventilation using either:

- windows or other openings (complying with the $5 \%$ rule); or

- high-level trickle ventilators located through the external wall where the distance between the external wall and opposing wall is less than six metres.

For kitchens, bathrooms, toilets, and laundries without an external wall, moisture must be ventilated to the outside by natural ventilation by using either:

- a passive stack ventilator designed to extract a continuous airflow through the surrounding habitable spaces;

- high-level trickle ventilators located within the external wall;

- permanent openings for airflow between the surrounding habitable spaces and the kitchen, bathroom, toilet, or laundry or no less than $5 \%$ of the combined floor area of the indoor environments, and not compromising privacy; or 
- a combined distance of the habitable space and the kitchen, bathroom, toilet, or laundry measured between the external wall and the furthest opposing wall of less than 10 metres.

Trickle ventilators are another means of ventilation. These are devices that have an opening to the outside. Clause G4 states that trickle ventilators shall:

- have an opening of no less than $2000 \mathrm{~mm}^{2}$ equivalent aerodynamic area;

- be located to minimise draughts;

- be secured to keep pests and insects out;

- have acoustic attenuation, if required by NZBC G6 Airborne and Impact Sound;

- be controllable and closable in all conditioned spaces;

- be installed in household units, providing they do not contain mechanical supply ventilation;

- have the sum of the equivalent aerodynamic area greater than the sums of the equivalent area of the passive stack ventilator(s), if installed in a household unit;

- have the equivalent aerodynamic area, based on the number of occupants, for the indoor environment as specified by Clause G4; and

- have, where high and low-level trickle ventilators are required, the high and lowlevel trickle ventilators of approximately the same equivalent aerodynamic area and separated by a minimum of one metre. High-level trickle ventilators are located in the top half of the wall. Low-level trickle ventilators are located in the bottom half of the wall.

Clause G4 also specifies the ventilation requirements for where both natural ventilation and mechanical ventilation are used. This describes what standards the installed equipment and designed outdoor air supply must comply with, as well as design considerations such as where outdoor air intakes should be located. It includes consideration for potential fire hazards. It states that for bathrooms, toilets, and laundries in buildings containing household units and accommodation units, extract ventilation must:

- be installed in a fire separated shaft, and

- have the branch connection to the common extract duct via a fire shunt of $900 \mathrm{~mm}$ in height, and 
- be ducting made of non-combustible material, unless the common extract duct is the only duct in the fire separated shaft.

\subsection{New Zealand Building Code: E3 Internal Moisture}

Clause E3 of the Building Code aims to safeguard people against illness, injury, or loss of amenity from the accumulation of internal moisture, and to protect household units and other property from damage caused by free water from another household unit in the same building. It uses NZS 4214:2006.

Clause E3 requires buildings be constructed to avoid fungal growth or the accumulation of contaminants on building elements, free water overflow penetrating to an adjoining household unit, and damage to building elements caused by moisture. There are six moisture performance goals for buildings:

- an adequate combination of thermal resistance, ventilation, and space temperature must be provided to habitable spaces, bathrooms, laundries, and other spaces where moisture may accumulate or be generated;

- free water from accidental overflow from sanitary fixtures or appliances must be disposed of in a way that avoids loss of amenity or damage to household units or other property;

- floor surfaces of any space containing sanitary fixtures or appliances must be impervious and easily cleaned;

- wall surfaces adjacent to sanitary fixtures or appliances must be impervious and easily cleaned;

- Surfaces of building elements likely to be splashed or become contaminated in the course of the intended use of the building must be impervious and easily cleaned; and

- surfaces of building elements likely to be splashed must be constructed in a way that prevents water splash from penetrating behind linings or into concealed spaces.

Clause E3 provides acceptable solutions for prevention of fungal growth and dealing with overflow and watersplash. Fungal growth is avoided by minimising internal condensation. 
This is done by maintaining the correct balance between interior temperature and ventilation. Insulation assists in maintaining interior temperatures at a suitable level.

The NZBC does not specify minimum heating requirements except for where a significant proportion of the current residents are elderly and early childhood centres. Occupants determine their methods and levels of heating. To control condensation in winter, it is typically necessary and sufficient to keep internal temperatures $5^{\circ} \mathrm{C}$ to $7^{\circ} \mathrm{C}$ above exterior temperatures in a ventilated space. Necessary thermal resistance and construction requirements are also outlined, while ventilation should comply with Clause G4.

For housing that is not air-conditioned, condensation should be controlled using condensation collection channels. These either discharge the water outside or are of sufficient capacity to hold the water, without overflowing, until it evaporates. These condensation channels, as well as drainage outlets, must be able to be cleaned.

If a sanitary fixture is located where accidental overflow could damage an adjoining household unit, containment and a floor waste must be provided. Containment maybe achieved by using impervious floor coverings which are continuous and coved or joints sealed where they meet the wall.

Dealing with watersplash requires impervious and easily cleaned surfaces. Examples of impervious linings and finishes include:

- integrally waterproof sheet material with sealed joints;

- ceramic or stone tiles having $6 \%$ maximum water absorption, waterproof grouted joints, and bedded with a suitable adhesive;

- cement-based solid plaster or concrete with a steel trowel or polished finish;

- cork tile or sheet sealed with waterproof applied coatings and with sealed joints;

- monolithic applied coatings with a polished non-absorbent finish; and

- a timber or timber-based product sealed with waterproof coatings.

Clause E3 states all shower spaces must have impervious floor and wall finishes. This is to help minimise potential water damage in particularly vulnerable areas. 
Alister Stubbe 


\section{Chapter 4: BRANZ Data}

\subsection{Background}

The information collected by BRANZ was part of the 2015 House Condition Survey. It recorded changes in moisture in around 70 homes around the country. Measurements of temperature and relative humidity were collected along with information about the sensor. Measurements and recordings of the physical environment were also recorded. This included room size, ventilation systems, and data on door and window opening angles.

\subsection{Collected Data}

As a companion to the BRANZ House Condition Survey, information was collected on windows and doors. This included whether they were open, and what angle they were positioned at. At the time of this research, much of this information is unusable, either because the data still needs to be processed and collated, or because the sensors were installed incorrectly. This makes it important to fully consider the potential consequences of additional ventilation caused by open windows and doors.

For this study, the data names were formatted as [Location Abbreviation] [Building Number]. For example, the sixth house to be recorded in Dunedin was called 'DN6'. Where there was more than one bathroom, this was indicated by a decimal point and value. For example, the second bathroom in house DN6 was called 'DN6.2'. Bathrooms were randomly assigned as either the first or second bathroom. This did not reflect the bathroom size or any other factors.

Data collected about the temperature and relative humidity are both in usable states. These recordings updated each time a change was recorded, including the time this change took place. For many of the houses there is data on the length and width of each recorded room.

\subsection{House Condition Survey Information}

The House Condition Survey included results on ventilation, heating and mould in New Zealand houses (White \& Jones, 2015). Although nearly all bathrooms had an openable 
window, $49 \%$ of main bathrooms did not have mechanical extract ventilation leading outside (Figure 2).

A. Openable windows

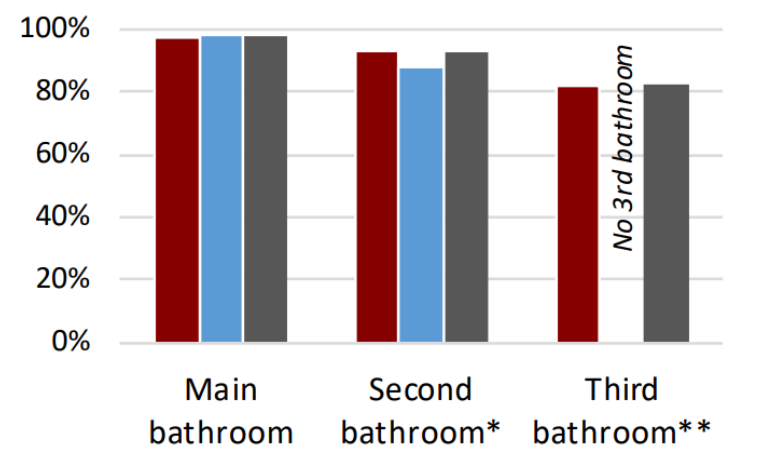

- Owned $\quad$ Rented
B. Mechanical ventilation (to outs ide)

$100 \%$

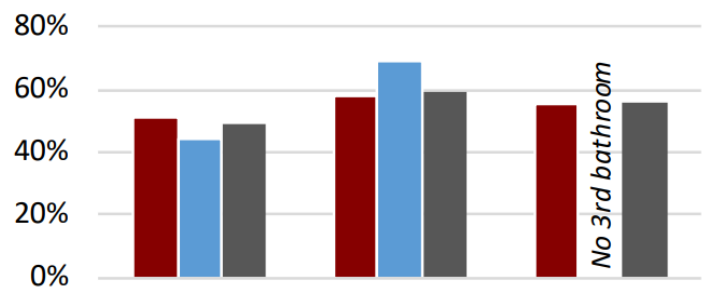

Main

Second

Third

bathroom bathroom* bathroom**

Figure 2 Presence of Openable Windows and Mechanical Ventilation in Bathrooms (White \& Jones, 2015)

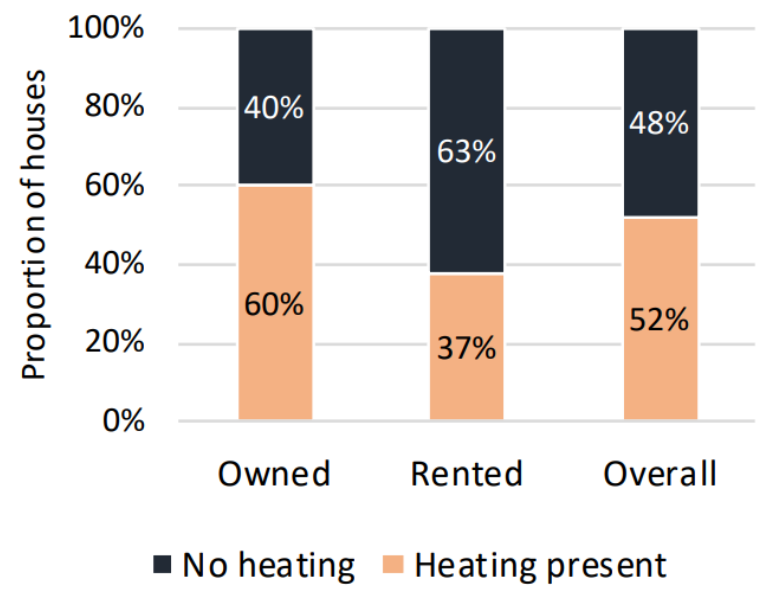

Figure 3 Houses with heating in the Main Bathrooms (White \& Jones, 2015)

had both (White \& Jones, 2015).
$5 \%$ of households did not usually heat living areas at all in winter, and almost half did not heat any occupied bathrooms in winter. $48 \%$ of households did not have heating in the main bathroom (Figure 3 ). $42 \%$ of main bathrooms in rental homes did not have heating or mechanical ventilation (Figure 4). In owner-occupied bathrooms, $24 \%$ did not have heating or mechanical ventilation while $33 \%$ 


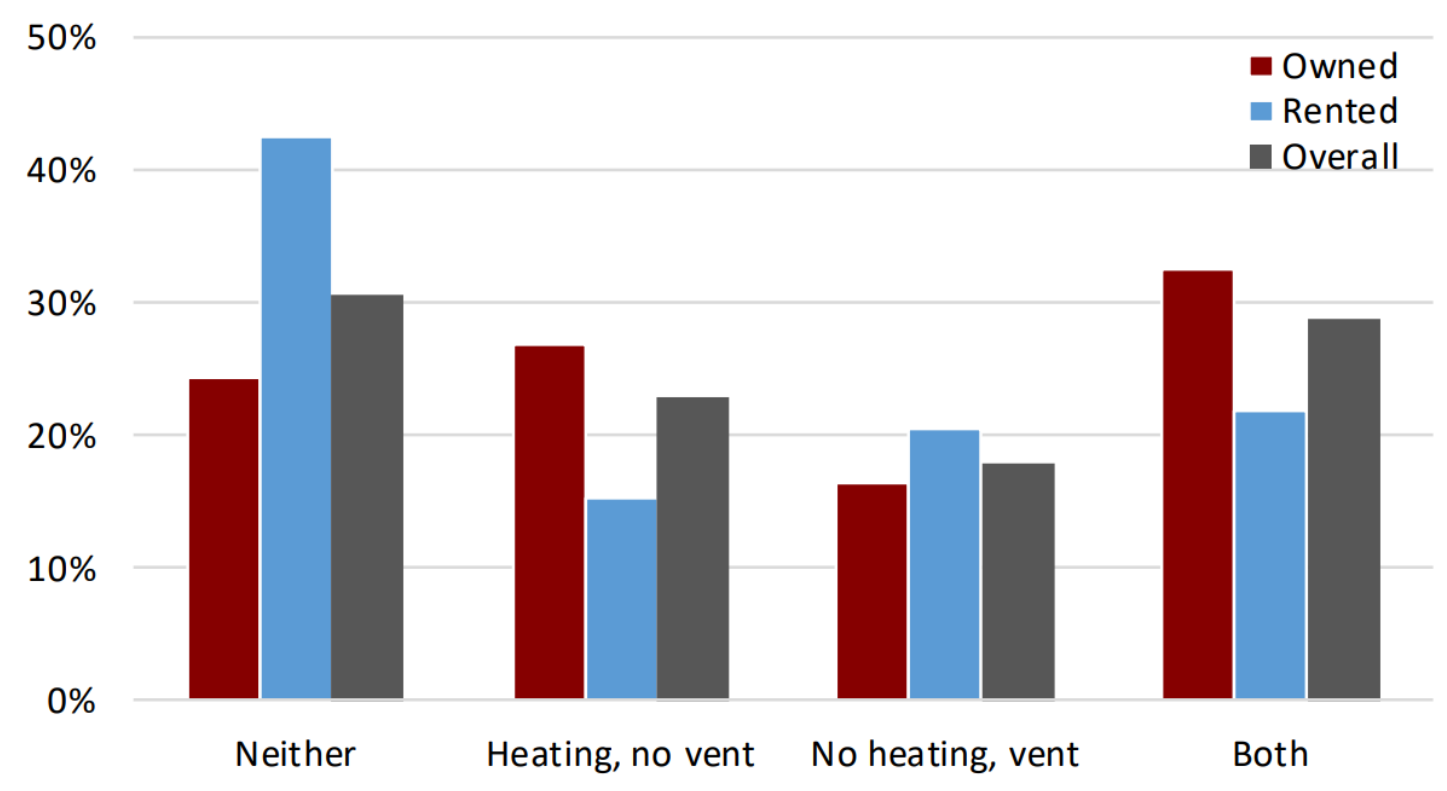

Figure 4 Presence of Heating and Ventilation in Bathrooms (White \& Jones, 2015)

Rental properties were twice as likely to smell damp than owner-occupied houses, and nearly three times as likely to feel damp. Mould was visible in nearly half of all houses surveyed and was slightly more common in rentals (White \& Jones, 2015).

\subsection{BRANZ Data Assessment}

Vicki White (BRANZ data analyst/reporter) and Manfred Plagmann (BRANZ senior physicist) reviewed the data collected over 2016 winter (White \& Plagmann, 2017). They found the median relative humidity recorded across all bathrooms over the study was $67 \%$, ranging between $43 \%$ and $88 \%$. One-third of the houses had a median relative humidity of $70 \%$ or higher, while six houses ( $10 \%$ of total participants) had a median relative humidity of $75 \%$ or higher. While peaks in moisture levels when the bathroom was in use were expected, these were expected to be brief.

Instead, a high proportion of houses had a relative humidity above $65 \%$ at least half the time. These levels of humidity over sustained periods of time increase the risk of problems with condensation and mould (White \& Plagmann, 2017).

Temperature is also a key factor. A lower temperature increases the chances of condensation and mould developing, particularly if surfaces are cold when moisture is generated. The average (median) temperature in the participants' bathrooms was $16^{\circ} \mathrm{C}$, but this ranged from a low of $8^{\circ} \mathrm{C}$ to a high of $20^{\circ} \mathrm{C} .48 \%$ of households had a median 
temperature of less than $16^{\circ} \mathrm{C}$, and $19 \%$ had a median temperature less than $14^{\circ} \mathrm{C}$. This means that for $50 \%$ of the study period, these bathrooms were colder than levels recommended for a healthy indoor environment (White \& Plagmann, 2017). It is important to note this study took place during winter and likely represents these houses at their coldest.

Considering the relative humidity data and the temperature data demonstrates which houses are particularly susceptible to mould and condensation. Ten houses ( $16 \%$ of participants in the study) had both median temperatures in the coldest $25 \%\left(8^{\circ} \mathrm{C}-14^{\circ} \mathrm{C}\right)$ and median relative humidity levels in the highest $25 \%$ ( $72 \%-80 \%$ relative humidity) of all houses surveyed. These houses are at significant risk.

Study participants were asked about their window and fan usage during and after taking a bath or shower. The responses show most people ventilate the bathroom, with $73 \%$ always ventilating the bathroom while showering and bathing, and $70 \%$ of people doing so afterwards. Extractor fans were the most common means of ventilation, followed by opening windows. $27 \%$ of participants did not have an extractor fan in their bathroom, while $2 \%$ of people did not have openable windows.

Despite these positive responses, results still demonstrated instances of high, sustained relative humidity. Some of the highest recorded levels were in bathrooms where occupants reportedly 'never' ventilated while taking a bath or shower, as would be expected. However, some houses reaching similar relative humidity levels had occupants claiming they 'always' ventilated their bathroom. Overall there were no significant differences in the median relative humidities of bathrooms based on occupant-reported ventilation habits. Similarly, comparing temperature data with information collected in the House Condition Survey about heating systems in bathrooms suggests there was no significant difference in the average temperature of bathrooms with and without heating available.

This all suggests that, where systems are available to help control moisture in the bathroom, they are not being used sufficiently or effectively and/or are not functioning adequately. That most people reported good practice in ventilating their bathroom before and after showering could suggest they think they are doing the right thing. However, recorded data 
shows their actions are not sufficient for maintaining a healthy environment and minimising the risk of condensation and mould (White \& Plagmann, 2017).

Potential steps for further analysis include understanding the drivers behind the relative humidity levels and temperature recorded, as well as how these relate to occupant ventilation and heating behaviour.

\subsection{Adapting BRANZ Data for use in this Study}

The BRANZ House Condition Survey (HCS) has been carried out every 5 years since 1994. Over time the survey has expanded in scope, looking at a wider range of houses and collecting more information. The data was collected with the intent of reporting on how New Zealand homes are performing.

However, analysing the performance of moisture extraction in bathrooms was not the express focus of the measurements. It measured relative humidity which compares the moisture in the air with the amount which air at that temperature could hold. As this changes with temperature, absolute humidity also has the advantage of being temperature independent. As this current study involved identifying periods where moisture was actively being introduced and removed from the space, regardless of the current temperature, absolute humidity was used instead of relative humidity.

\subsection{Additional Considerations}

BRANZ also acquired local weather data for the survey period and areas from NIWA. This enabled weather data to be used to verify the impact the outdoor climate had on moisture changes. Combining information on building orientation, number of windows, temperature, relative humidity, wind speed, and wind direction provided a clear indicator of the impact the external environment has on the bathroom.

BRANZ has highlighted that the impact of occupants on the data is an important element for future studies to consider (White \& Plagmann, 2017). This study aimed to contribute towards this, providing information about periods of rapid change in absolute humidity (see section 5.3 Moisture Event) within residential bathrooms. This could be compared to selfreported use of moisture sources such as showers, providing information on how many of the periods of rapid change in absolute humidity that take place in most bathrooms are 
Alister Stubbe

caused by occupant activity. It may also provide an indicator of the accuracy of the selfreported information. 


\section{Chapter 5: Methodology Design}

\subsection{Overview}

The aim of this study was to identify and analyse periods of rapid change in absolute humidity in the bathrooms of New Zealand homes, providing insight into how moisture sources are currently being used. The data from one bathroom was tested and briefly analysed. This initial data test informed the process carried out to analyse the rest of the data. A method for visualising series periods of rapid change in absolute humidity and other relevant information was developed. Different criteria for determining series of rapid changes were tested. Series of measurements that met these criteria were named moisture events.

Different patterns of moisture events were identified and defined as a certain class: episodes, increases, decreases, and combinations. The mean and median episodes for each bathroom were calculated. This included information on the absolute humidity at the start, peak, and end of each episode as well as the total episode length and the length from the episode start to peak.

Moisture events were filtered according to size and length, eliminating short moisture events that only introduced or removed small quantities of moisture from the indoor environment. Days were broken into four hour periods. The filtered moisture events that took place during each of these periods was recorded. Days that shared similar patterns in moisture events (most of which were episodes) were grouped. The most common of these groups were referred to as typical days. Typical days provided information on how often episodes were likely to occur and at what times.

Combining the mean and median episode information with the typical days for each bathroom allowed the bathrooms to be compared. The bathrooms were grouped based on the available information such as the number of bathrooms in each house, the bathroom size, and whether mechanical ventilation was present. 


\subsection{Objective}

This analysis aimed to study how moisture content in at-risk areas of New Zealand homes changes over the course of the day. It provided information on the impact of each period of rapid change in absolute humidity (see 5.3 Moisture Events), demonstrating how long these lasted, how much moisture was introduced, and how often they took place. This also helps to demonstrate how occupants typically operate their bathrooms, showing how often moisture sources are typically active and the times they are typically used.

This was done with the intent of reviewing how well the NZBC is performing in terms of preventing damp and unhealthy buildings. It provides a starting point for a comprehensive review of whether the ventilation requirements of Clause G4 of the NZBC result an adequately healthy environment and whether they sufficiently take the occupants' behaviour into account.

\subsection{Moisture Events}

\subsubsection{Defining a Moisture Event}

A 'moisture event' describes a period where the indoor environment experiences a rapid change in humidity. This is used as an indication of large amounts of moisture being introduced to the indoor environment, such as by a shower, or removed from the indoor environment, such as by an extract fan. Chapter 2: Literature Review demonstrated the importance of humidity in maintaining a healthy environment. Moisture events can be used as indicators for this, showing how much moisture is being introduced as well as how long it takes for that moisture to be removed. Comparing moisture events between houses indicates how New Zealand homes are performing at maintaining a healthy environment, how long it takes for this environment to stabilise after moisture has been introduced, and which factors have the largest impact on these events.

\subsubsection{Rate of Change Criteria}

As the evaluation of moisture events is based on comparing results from different houses, the exact rate of change used to define a moisture event was irrelevant. The most important consideration was for it to be consistent for all houses, while still identifying as many potentially relevant measurements as possible. This means the percentage used needed to be small enough to be reached whenever a moisture source was actively introducing 
moisture to the environment, while large enough that natural changes in humidity levels throughout the day were ignored. The selected rate of change needed to be able to do this for all houses analysed.

\subsubsection{Data Considerations}

\subsubsection{Absolute Humidity vs Relative Humidity}

The data provided by BRANZ measured relative humidity. As discussed in 2.4 Mould and Moisture Indicators, this is a useful indicator of whether the indoor environment is at risk of mould and moisture damage. However, in this case, the analysis aimed to identify how much moisture was being introduced by moisture sources. To achieve this, absolute humidity has been used rather than relative humidity. This allows moisture events to be identified purely based on how much moisture was introduced to the indoor environment and is not influenced by changes in temperature.

\subsubsection{Measurement Intervals}

The data provided by BRANZ was not measured at regular intervals. This may have affected how moisture events were identified. The rate of change between two measurements will change depending on the time between each of them, even if the change in absolute humidity is the same.

\subsubsection{Percentage Change vs Quantity Increase}

Moisture events were calculated based on whether a measurement was a certain percentage higher than the previous measurement. The alternative was to calculate exactly how much the absolute humidity had increased/decreased by, and count a recorded measurement as being part of a moisture event if it exceeded a certain value. As the size of moisture events varied between houses, the value would need to be changed to identify all notable changes in absolute humidity. Basing moisture events on a constant percentage helped ensure the results were comparable. All sudden changes in absolute humidity were identified, regardless of whether they were large or small. 


\subsection{Methodology Basis}

\subsubsection{Developing from the Literature}

Temperature and humidity can be used as indicators for potential moisture damage.

Reviewing the rate these change over time provides insight into how the indoor

environment deals with moisture. A sudden spike in humidity, such as from a shower being

turned on, allows comparison between the room at base moisture levels (discussed in

Typical Daily Indoor Temperature vs Outdoor Temperature) and peak moisture levels (the

highest level of absolute humidity reached during an event).

After the shower is turned off, moisture levels naturally begin to lower as moisture spreads outside and through the rest of the house. This rate of change varies depending on what moisture control systems are being used. For example, trickle vents (or other continuous ventilation systems) introduce a continuous amount of outdoor air to the indoor environment, increasing the number of air changes per hour and helping to stabilise the indoor humidity compared to the outdoor humidity.

However trickle vents have been measured to only reduce moisture by $4.5 \%$ humidity in a 10 minute period in New Zealand homes (McDowall, 2017). It would take hours for trickle vents to remove the moisture introduced by a shower or other large moisture event. Openable windows provide a more direct means of controlling moisture. Opening these for 10 minutes after a moisture event helps remove a large portion of the introduced moisture, reducing airborne moisture by $14 \%$ (McDowall, 2017).

Clause G4 of the Building Code states New Zealand buildings must comply with NZS 4303:1990. This means residential bathrooms must include ventilation providing a minimum mechanical extract air flow of $25 \mathrm{~L} / \mathrm{s}$ intermittent, $10 \mathrm{~L} / \mathrm{s}$ continuous, or to provide openable windows (Standards New Zealand, 1990). By tracking how the indoor environment of New Zealand houses vary during the day over an extended period the effectiveness of these ventilation standards can be analysed. Almost all bathrooms included in the 2015 House Condition Survey had an openable window (White \& Jones, 2015), meeting the NZBC requirements. If moisture is not being effectively removed from the measured spaces this may indicate a problem with current building practices and how occupants control the indoor environment. 


\subsubsection{What to Measure}

Moisture indicators can be used to provide information on potential moisture damage, highlighting which buildings are either most at risk or have already been damaged. As discussed in 2.4 Mould and Moisture Indicators, these include visible mould damage, active water leakage, building materials detected to be moist, relative humidity exceeding $80 \%$ in drill-hole measurements, or a material sample showing active microbial growth (Annila et al., 2017).

These indicators show which buildings are already affected by moisture damage, but do not necessarily show which buildings are at risk. A better option was to look at measurements of the original cause of the issue, analysing moisture sources and the indoor climate as a whole.

\subsubsection{Adapting to Available Data}

With the data provided by BRANZ (Chapter 4: BRANZ Data) combined with weather data provided by NIWA, a range of relevant measurements were available for analysis.

\begin{tabular}{|l|l|l|}
\hline \multicolumn{1}{|c|}{ Data Type } & \multicolumn{1}{|c|}{ Available Data } & \multicolumn{1}{c|}{ Description } \\
\hline \multirow{4}{*}{ House } & Temperature & Recorded indoor temperature $\left({ }^{\circ} \mathrm{C}\right)$. \\
\cline { 2 - 3 } & Relative humidity & Recorded indoor relative humidity $(\%)$. \\
\cline { 2 - 3 } & Time & The time measurements were taken. \\
\cline { 2 - 3 } & Location & The region of New Zealand where the house is located. \\
\cline { 2 - 3 } & Room Dimensions & The room length and width $(\mathrm{m})$. \\
\hline \multirow{4}{*}{ Weather } & Temperature & Recorded outdoor temperature $\left({ }^{\circ} \mathrm{C}\right)$. \\
\cline { 2 - 3 } & Relative Humidity & Recorded outdoor relative humidity $(\%)$. \\
\cline { 2 - 3 } & Wind & Recorded wind speed $(\mathrm{m} / \mathrm{s})$ and direction. \\
\hline
\end{tabular}

Table 5: Recorded Data

This set of data did not include information on door or window openings as the available information is not yet in a usable state for most houses. Some houses had additional information available on the room dimensions, presence of windows, and presence of mechanical ventilation. This is described in 7.1.3 Selecting Analysis Period.

\subsubsection{Access to Programs}

Data sets were taken from an SQL database and converted into graphs using Excel for further analysis and to visually highlight areas of interest consistently between houses. Multiple templates were created using Excel to process all measurements.

33 


\subsection{Intended Output}

\subsubsection{Overall Goal of Proposed Methodology}

The goal of the methodology was to isolate periods of rapid change in absolute humidity taking place within each house. By completing this for multiple houses, the length and absolute humidity levels for moisture events in different bathrooms could be compared and analysed. This provided a better understanding of the conditions bathrooms undergo while moisture sources are active.

\subsubsection{Initial Data Analysis}

Using the data provided by BRANZ, temperature and humidity were mapped for each house, showing how they change over the course of each day and at what rate. These were required to be presented in a way that highlighted periods of rapid change (moisture events) and periods with no change. Moisture events needed to be immediately identifiable, with information available on the start point, humidity peak, and end point. A brief analysis of these results was then carried out to identify areas of interest that required further analysis following the full-scale data processing. The effectiveness of the process was then reviewed and appropriate changes were implemented. This established a template to analyse all remaining houses.

\subsubsection{Full-Scale Data Processing}

Using the system established by the initial data test, all houses were processed. This provided a graphic representation of every measured day and extensive data on moisture events. It also helped to highlight any gaps in the data, such as a period from 3.00am to $9.00 \mathrm{pm}$ one day with no measurements, allowing further analysis to account for these anomalies. 


\subsection{Analysis Process}

\subsubsection{Step 1: Define Types of Moisture Events}

\subsubsection{Purpose}

To compare moisture events in different houses, moisture events were categorised according to how the absolute humidity changed. This allowed moisture events in the same group to be analysed in greater detail, noting how the rate of change varied between similar events.

\subsubsection{Method}

The data processing method established in 6.5.4 Creating a Data Analysis Template identified series of measurements with a rate of change of $3 \%$ or greater, classifying them as moisture events. These were categorised into groups based on whether the rate of change was positive or negative at each point of the event.

\subsubsection{Result}

Similar moisture events were identified and further analysed. Outliers, such as where only one example of a type of moisture event occurred, were taken into account. Regular moisture events of the same type were identified.

\subsubsection{Step 2: Identify Typical Days}

\subsubsection{Purpose}

To identify the moisture events most likely to have a large impact on the indoor environment and identify regular moisture events of the same type. This allowed potential trends between days to be identified.

\subsubsection{Method}

The data processing method established in 6.5.4 Creating a Data Analysis Template was used to analyse which moisture events occur each day. The established definition of moisture events was used to identify types of moisture events. Additional criteria then identified the events with the greatest impact. For example, the events that lasted the longest or resulted in large increases or decreases of absolute humidity.

These were grouped based on the time they occurred. Days with the same types of moisture event occurring at the same time were grouped. The groups representing the largest number of days were described as 'typical days'.

35

Methodology Design 


\subsubsection{Result}

This provided additional context for the analysis of moisture events, showing how they were distributed and how likely it is for them to occur. Recording the typical days provided a simple indication of how often each combination of moisture events (and subsequent quantity of moisture present in the indoor environment) was likely to occur.

\subsubsection{Step 3: Calculate Average Moisture Episode}

\subsubsection{Purpose}

Finding the mean and median absolute humidity for moisture events in each house provided clear, quantitative data. This allowed moisture events in each house to be compared.

\subsubsection{Method}

To calculate an average moisture event the same category of moisture event (as discussed in 5.6.1 Step 1: Define Types of Moisture Events) must be used. This analysis focused on a single category, but further research may consider carrying out this analysis for all categories.

\subsubsection{Result}

The behaviour of the selected category of moisture event was visually represented, showing how the length varied and by how much it increased and decreased at each stage. This provided data that could be grouped and compared to measurements from different houses.

This analysis permitted the calculation of the median and mean absolute humidity and length of each house's moisture events to be calculated.

\subsubsection{Step 4: Compare Average Episode to Typical Days}

\subsubsection{Purpose}

To identify any differences or similarities between the houses being compared.

\subsubsection{Method}

Houses with similar moisture event behaviour were compared for event distribution and typical days, as described in 5.6.2 Step 2: Identify Typical Days. For example, these houses may have the same number of moisture events take place on the most frequently occurring typical days in each of them. 
Moisture in New Zealand Bathrooms

\subsubsection{Result}

This provided additional context for analysing trends and groups.

5.6.5 Step 5: Identify Trends and Groups between Different Houses

\subsubsection{Purpose}

To describe similarities and differences between the moisture events taking place in the houses.

\subsubsection{Method}

The mean and median episodes were divided into groups based on the information available for each house and the results discussed in 5.6.4 Step 4: Compare Average Episode to Typical Days. This included floor area, ventilation, number of bathrooms, and region. Groups with different patterns (for example, one bathroom compared to two bathrooms) may represent a trend in moisture event behaviour.

\subsubsection{Result}

This provided some indication of how moisture events typically behave in New Zealand houses as well as which factors may play the greatest role in affecting this, allowing these trends to be further explored. 
Alister Stubbe 


\section{Chapter 6: Initial Data Test}

\subsection{Aim}

The purpose of this test was to prepare the data for analysis and to better understand what information it could provide. This involved three primary objectives:

- identify periods of rapid change in absolute humidity as moisture events;

- present the data in a visual form that immediately informs the reader of the conditions inside the indoor environment each day; and

- review measurements from this house to confirm that these previous objectives have been met and to make appropriate changes to the analysis process before carrying out a full review.

\subsection{Testing a Single House}

Data from a Dunedin house containing information from the bathroom and bedrooms was used. This was provided by BRANZ in two SQL files. One file contained meta-data identifying each sensor, their location, and motion type. The other file contained one month worth of data in SQL statements containing over 70,000 measurements. Carrying out a test for processing these measurements on a single house was an important step in establishing whether this would be a viable process for the full data analysis.

A total of four sensors were placed in the house. These were positioned in:

- the master bedroom $\left(12.1 \mathrm{~m}^{2}\right)$ - Sensor code DN4.2;

- a young child's bedroom $\left(8.5 \mathrm{~m}^{2}\right)$ - DN4.5;

- the bathroom $\left(4.8 \mathrm{~m}^{2}\right)$ - DN4.4; and

- the main living space with one window $\left(9.7 \mathrm{~m}^{2}\right)$ - DN4.3.

From here, the data was imported into Excel. This meant changes in temperature and moisture during the day could be quickly and visually expressed, allowing potential trends to be identified for further analysis. 


\subsection{Objective 1: Testing Required Moisture Event Level}

As established in 5.3 Moisture Events, the exact rate of change used to define a moisture event is unimportant. What matters is that it identifies periods where a moisture source is active, and is consistent in all analysed houses. The most effective way to determine whether a selected rate of change would be appropriate was to test it on different houses, selecting the lowest level that met these criteria in all of them.

\subsubsection{Examples of Different Moisture Event Levels}

\subsubsection{Presenting Moisture Events}

Using the data processing methods later described in this chapter, measurements where the percentage change in absolute humidity exceeded a specified value have been represented by black bars in exploratory data plots (e.g. Figure 5). For contrast, measurements where low levels of change took place ( $<0.03 \%$ for 20 or more minutes) are represented by yellow bars.

The moisture event also included the measurements directly before and after the first and last measurements to qualify. This helps to more accurately show when the moisture event started and stopped, and at what level. For example, if a measurement shows $8 \mathrm{~g} / \mathrm{m}^{3}$, the next measure shows $12 \mathrm{~g} / \mathrm{m}^{3}$, and the next measurement shows the peak at $13 \mathrm{~g} / \mathrm{m}^{3}$, knowing that the moisture event began with an increase from $8 \mathrm{~g} / \mathrm{m}^{3}$ to $12 \mathrm{~g} / \mathrm{m}^{3}$ is important when trying to analyse how the absolute humidity changes. However, only noting the first number to reach the specified rate of change would mean that the $8 \mathrm{~g} / \mathrm{m}^{3}$ measurement would not appear as part of the moisture event.

The first step was to define the appropriate rate of change at which an event was to be defined $-1 \%, 2 \%, 3 \%$ or $4 \%$. 


\subsubsection{1\% Rate of Change}

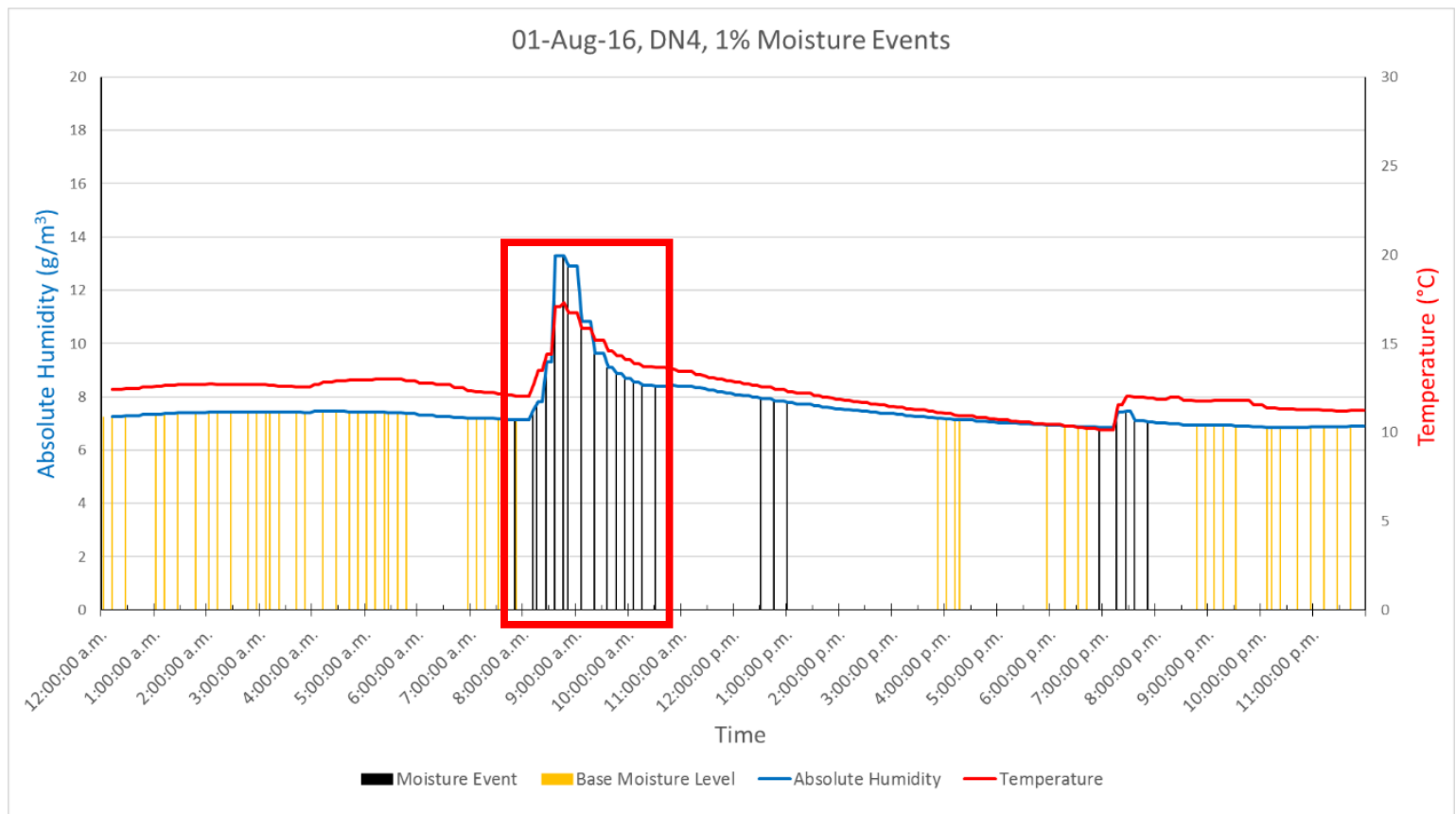

Figure 5, 1\% Large and Small Event

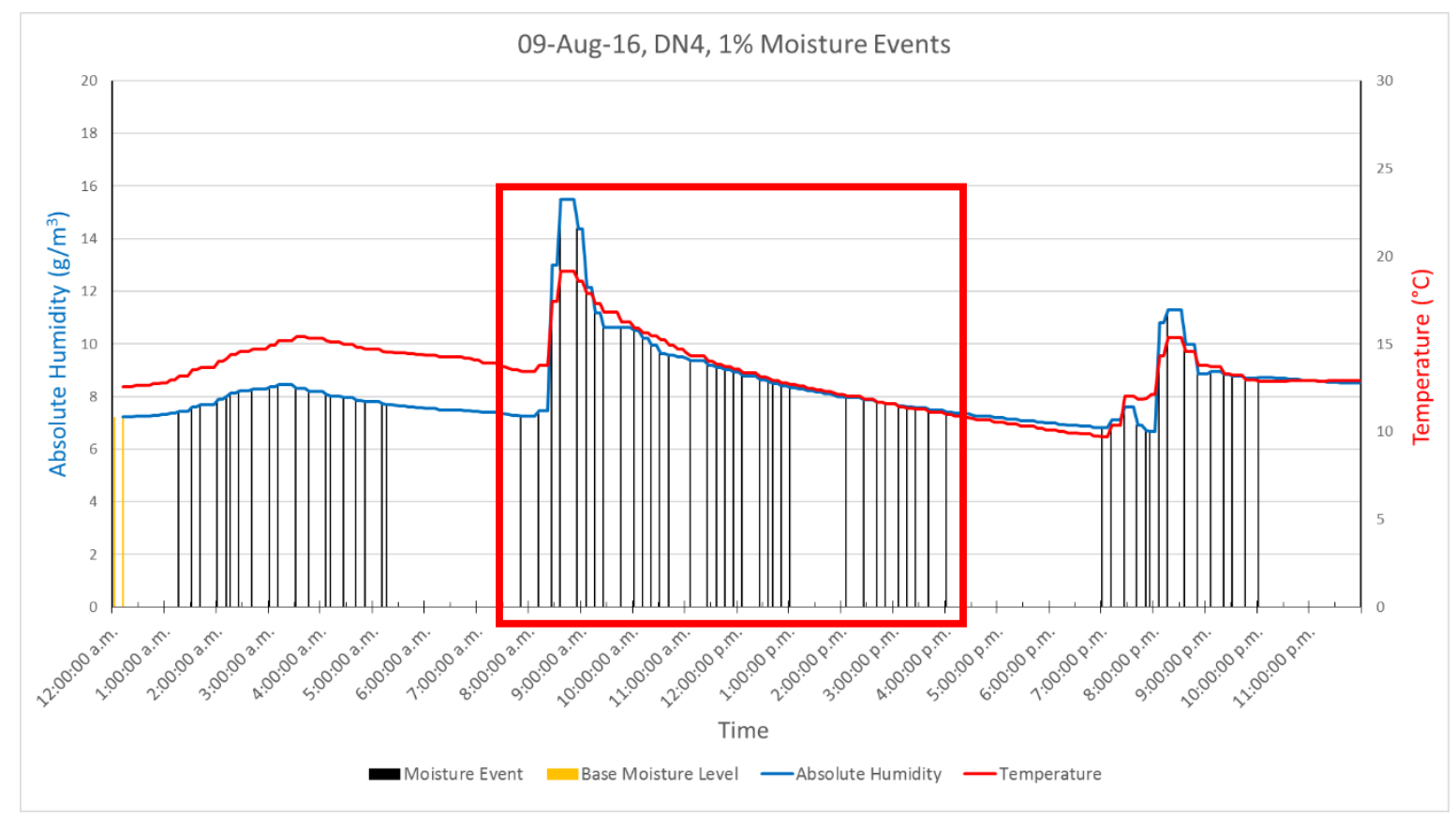

Figure 6, 1\% Long Event

At a rate of change of $1 \%$ all moisture events were identified, but other changes in absolute humidity were also incorrectly included. In Figure 5 the moisture event which started at 8.00am ended at 10.30am. However, a second moisture event was incorrectly detected 
from $2.00 \mathrm{pm}$ to $4.00 \mathrm{pm}$ while the decreasing absolute humidity briefly reaches the required rate.

In Figure 6 a change in absolute humidity in the morning that is unlikely to be caused by an active moisture source due to the steady rate of change over a long period, was considered to be a moisture event. The moisture event starting at 8.30am experiences the same issue as the event from Figure 5, registering a new series of measurements at $12.30 \mathrm{pm}$ as a moisture event after the original series of measurements had already finished and the absolute humidity had begun to decrease at a reasonably constant rate of change.

A rate of change of $1 \%$ was too low to define a moisture event.

\subsubsection{2\% Rate of Change}

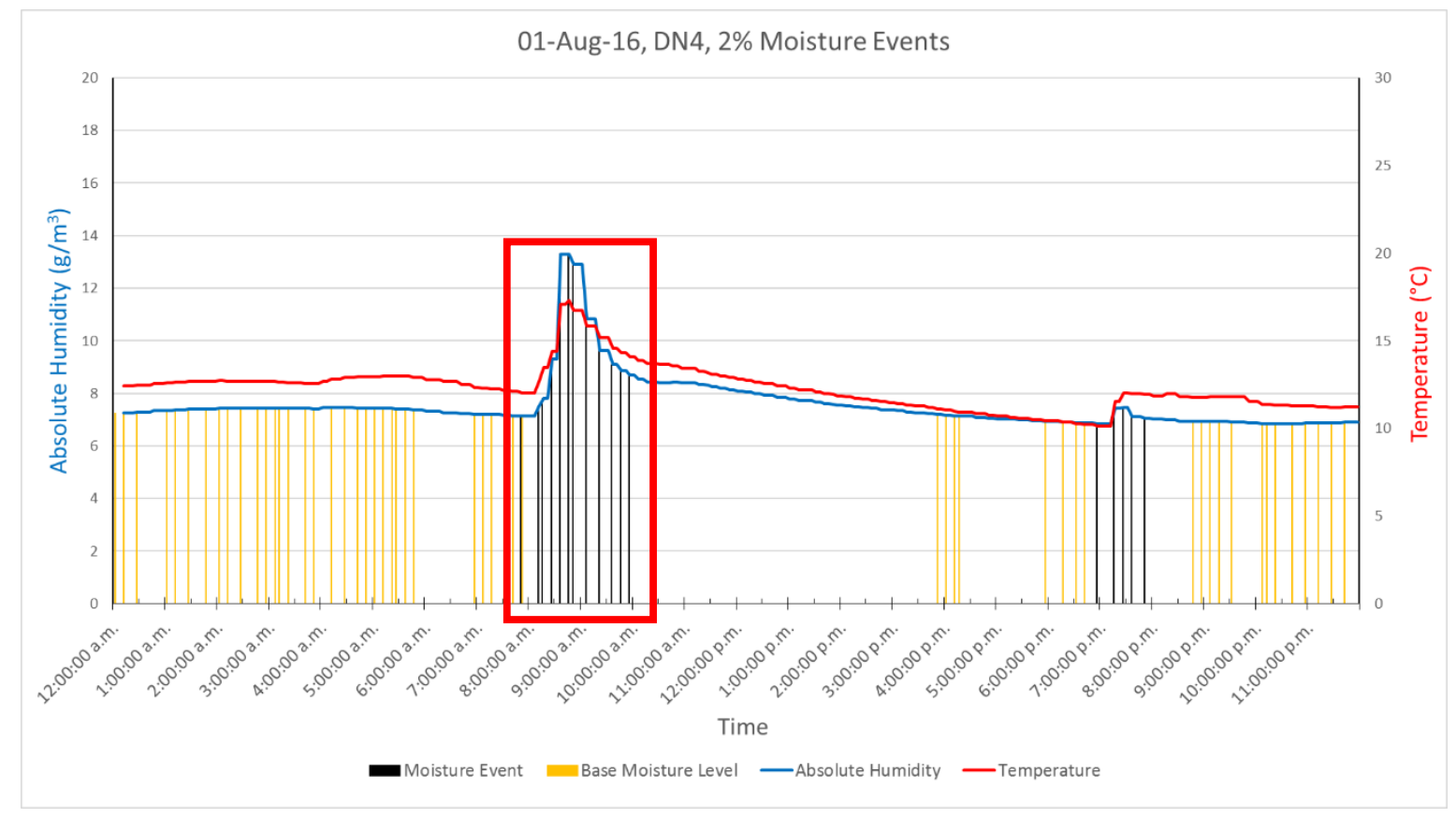

Figure 7, 2\% Large and Small Event 


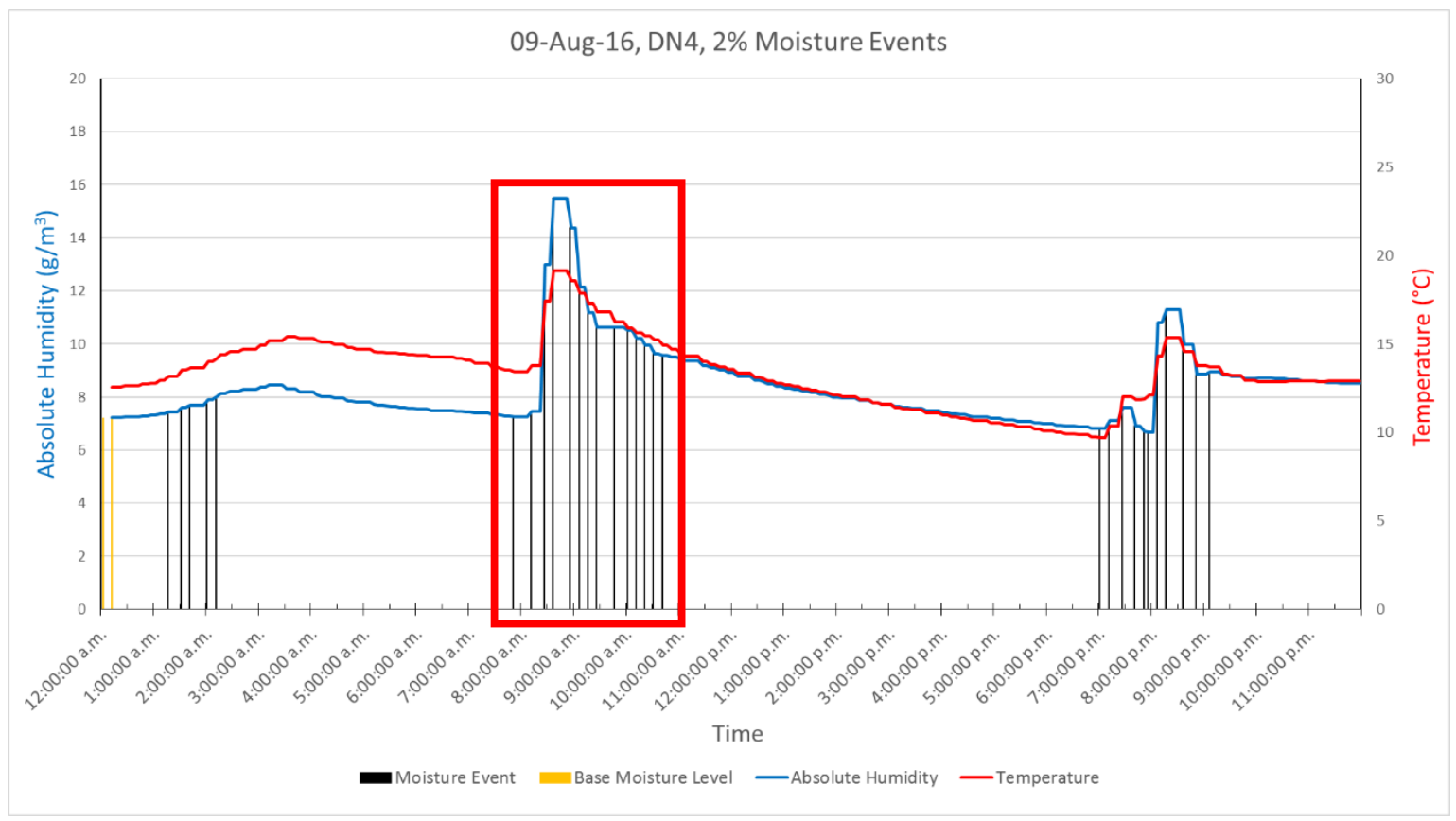

Figure 8, 2\% Long Event

At $2 \%$ both the large and small moisture events shown in Figure 7 were correctly identified. However, in Figure 8 part of the early morning change from 1.15am to 2.15am was still registering as a moisture event. This rate of change is helpful for identifying most moisture events but was still prone to incorrectly identifying events.

\subsubsection{3\% Rate of Change}

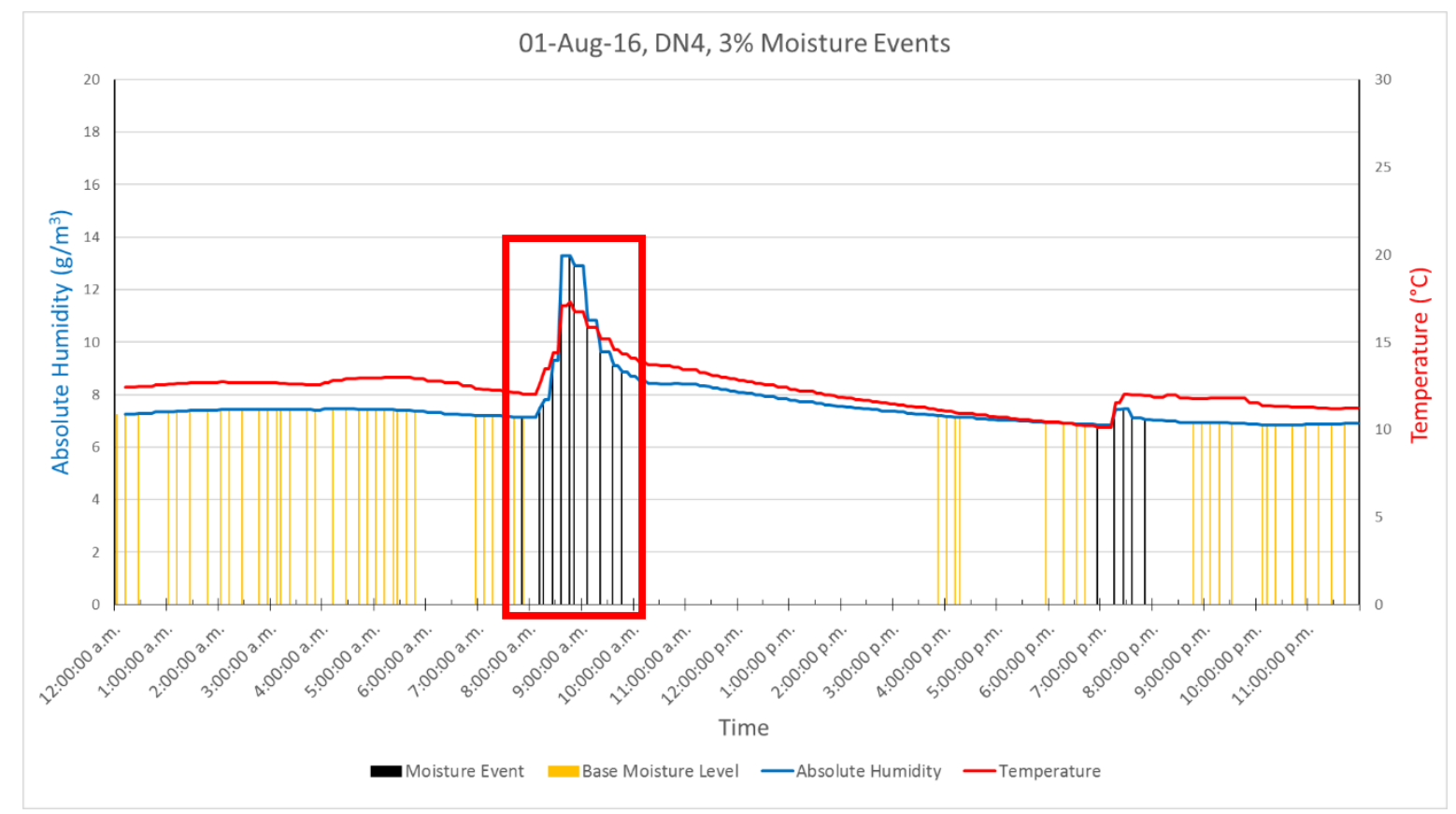

Figure 9, 3\% Large and Small Event 


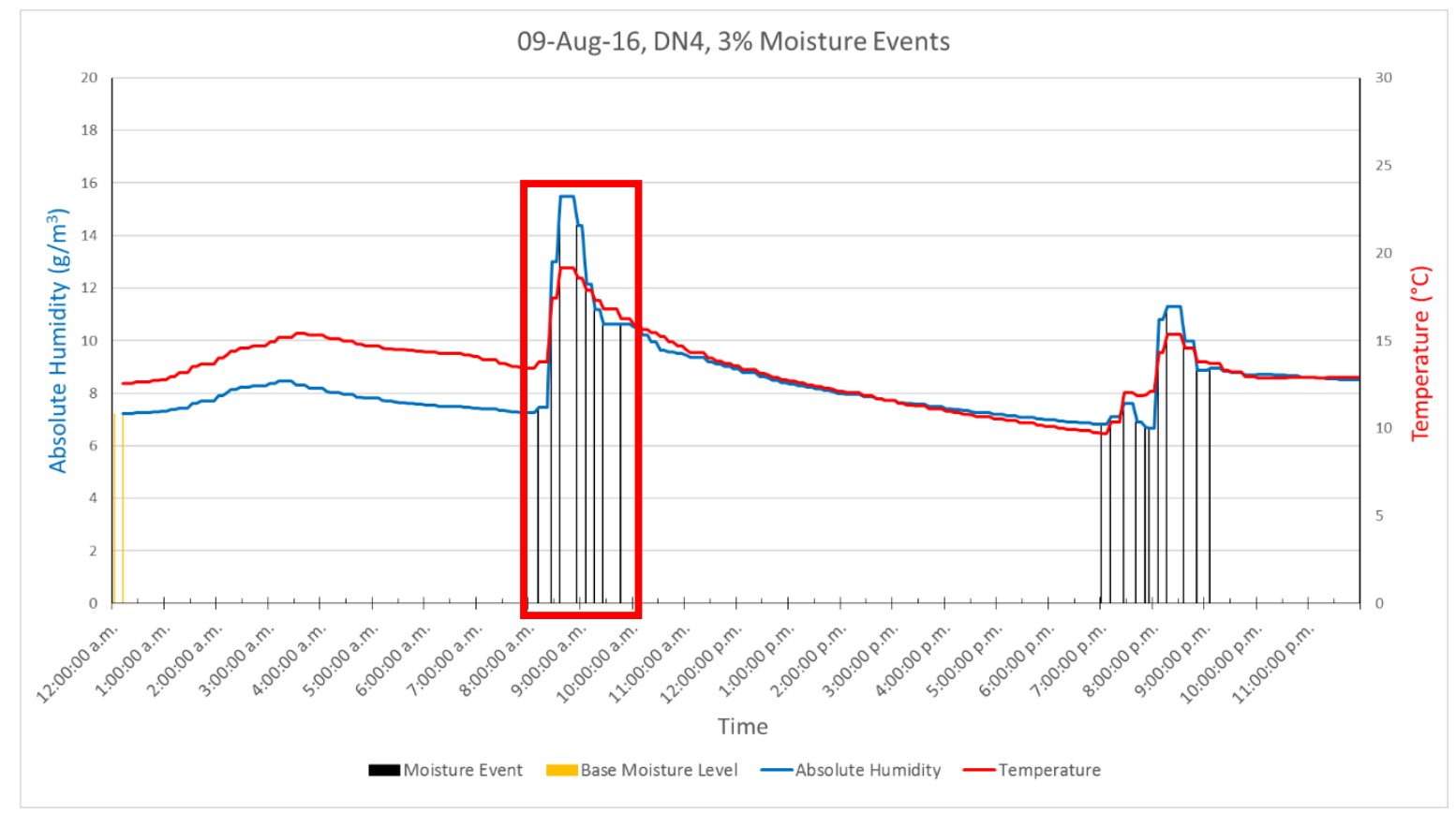

Figure 10, 3\% Long Event

At $3 \%$ the moisture events in Figure 9 continue to be accurately identified. The end of the $8.00 \mathrm{pm}$ moisture event occurs just as the absolute humidity began stabilising. The change that occurred in the early morning shown in Figure 10 was no longer identified as a moisture event, but both the 8.30 am event and 7.00pm event remain identified.

This level of rate of change correctly identifies most situations.

\subsubsection{4\% Rate of Change}

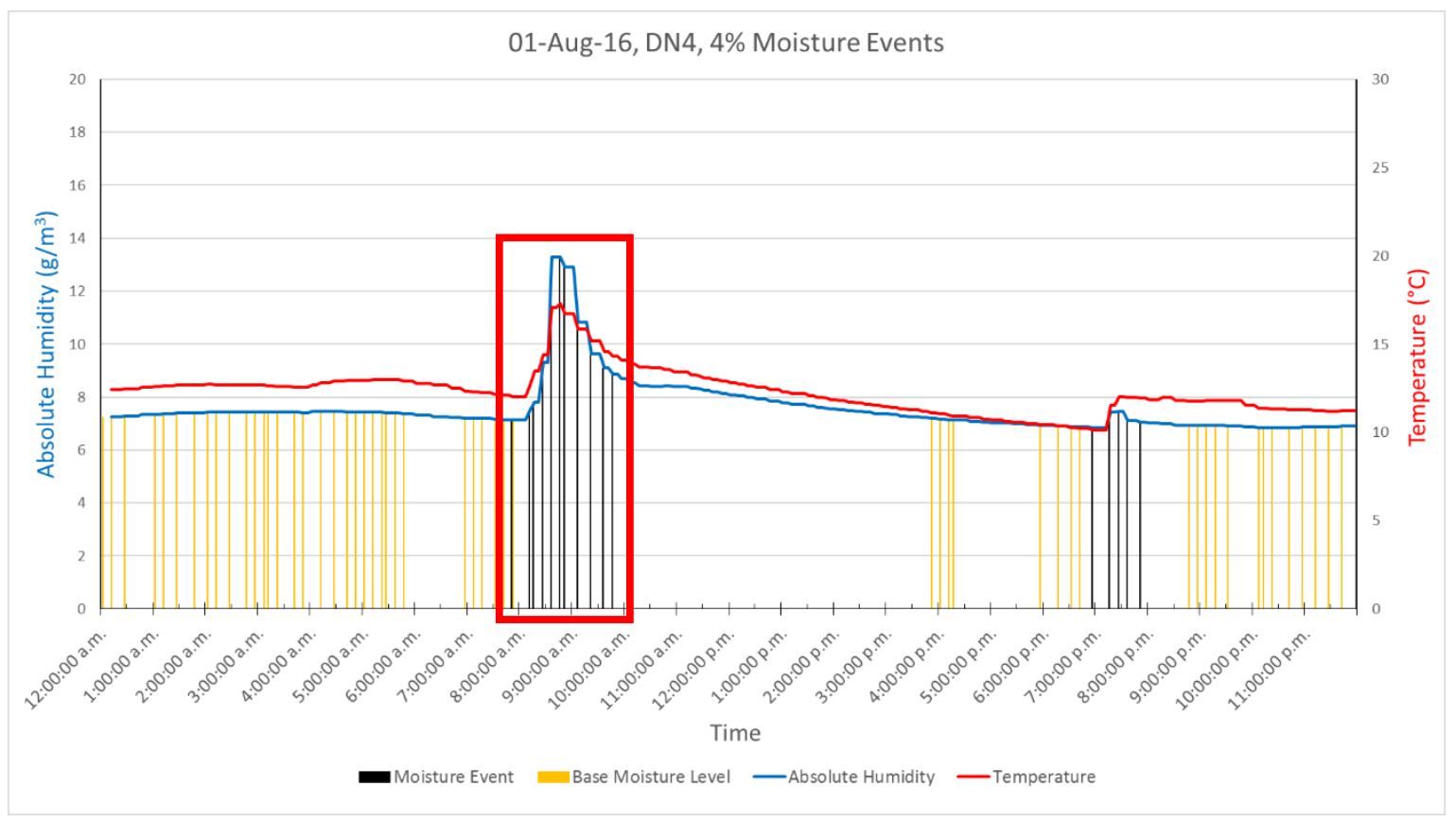


Figure 11, 4\% Large and Small Event

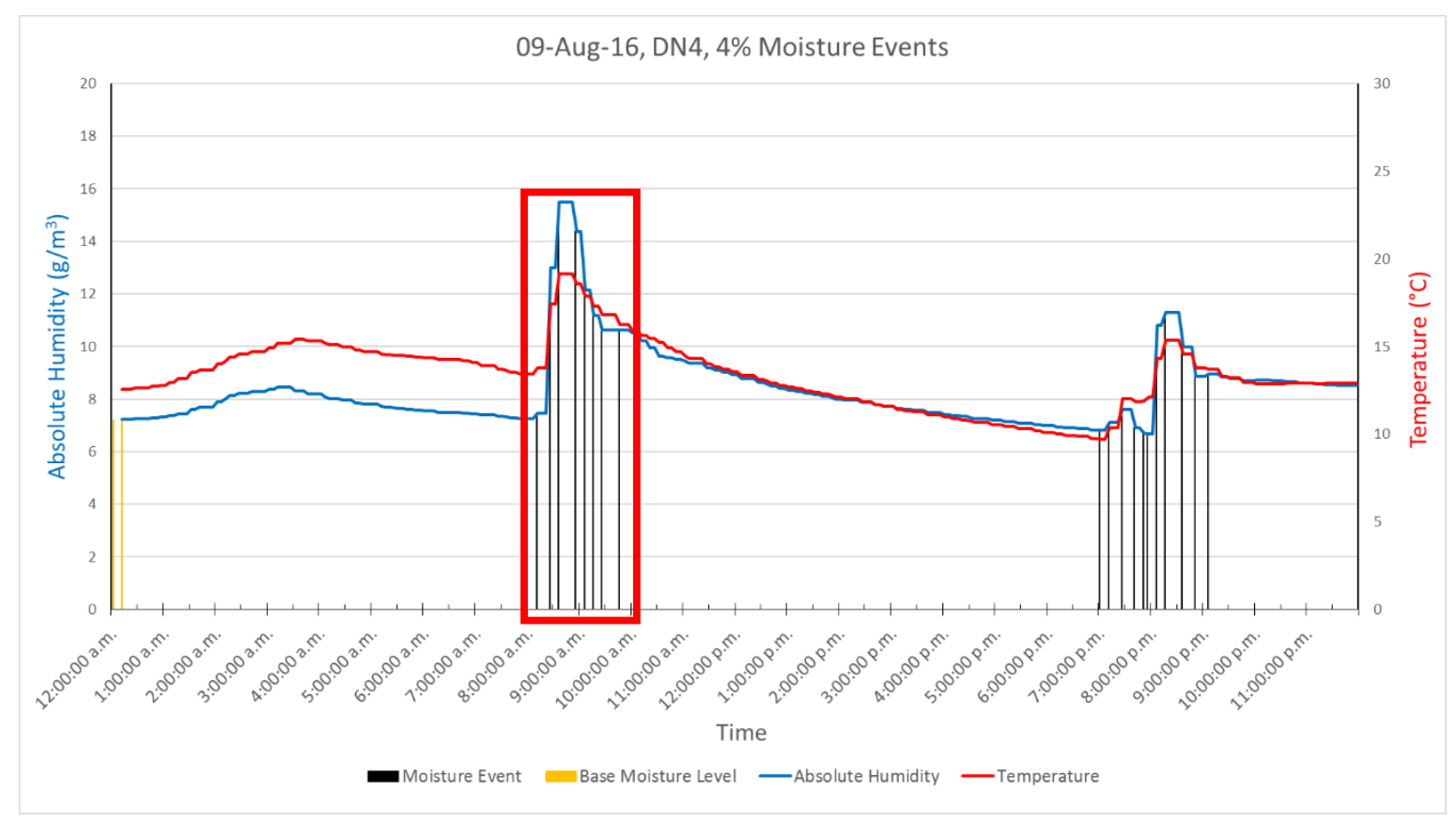

Figure 12, 4\% Long Event

As shown in Figure 11 and Figure 12, no major changes in identified moisture events take place when compared to a $3 \%$ rate of change. As the likelihood of incorrectly identified moisture events occurring was already greatly reduced, increasing the required rate of change to $4 \%$ simply increased the chance of moisture events being missed without providing enough of a benefit. A 3\% rate of change is an adequate level for identifying moisture events. 


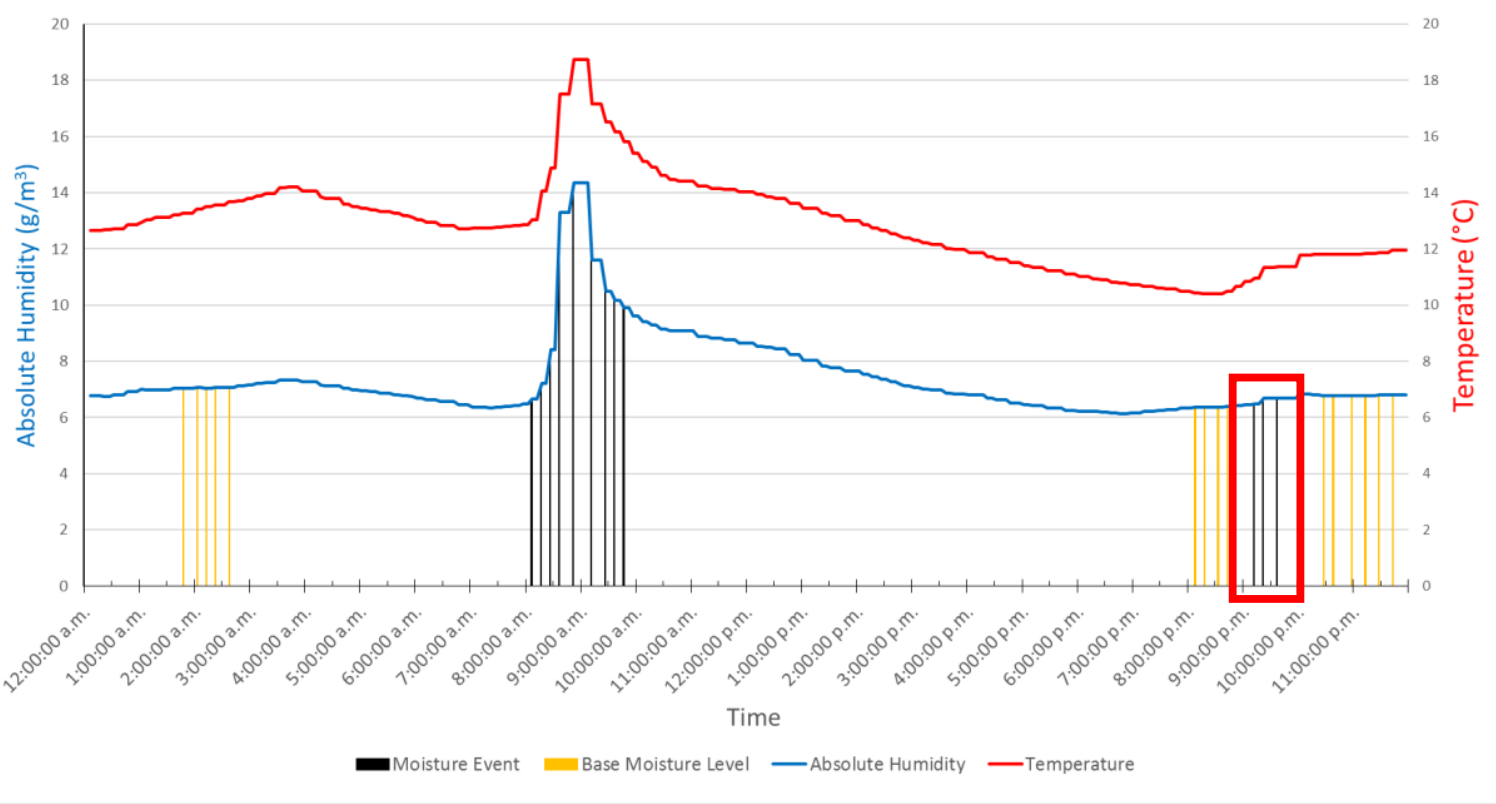

Figure 13 Potential Missed event

Figure 13 is an example of moisture events that may be missed at $4 \%$. The moisture event identified at 9.20pm shows a small increase in absolute humidity that just meets the criteria. Increasing the rate of change limit to $4 \%$ would cause this event to no longer qualify.

\subsubsection{Potential Moisture Events}

One of the risks of identifying moisture events using a constant rate of change level is that each house being analysed is unique. While the selected level of $3 \%$ was tested in a variety of houses for several different days, there was no reasonable way of ensuring this level would work for every single situation. While this identification system was designed to mitigate that risk as much as possible, such as by using percentage changes rather than the exact quantity of moisture, there were still situations where this was not enough.

The solution was to introduce a second class of moisture events: potential moisture events. These work by identifying a $2 \%$ rate of change rather than $3 \%$, providing additional context for all moisture events. For example, if two separate moisture events are identified as having occurred within a short period, in reality, there may have only been one moisture event which happened to contain a brief lull in absolute humidity. While this is unlikely (the measurements added before and after an event would mean that this lull would need to take place over several measurements to not be counted), including potential moisture events helped identify any events that may need to be considered a single event. 


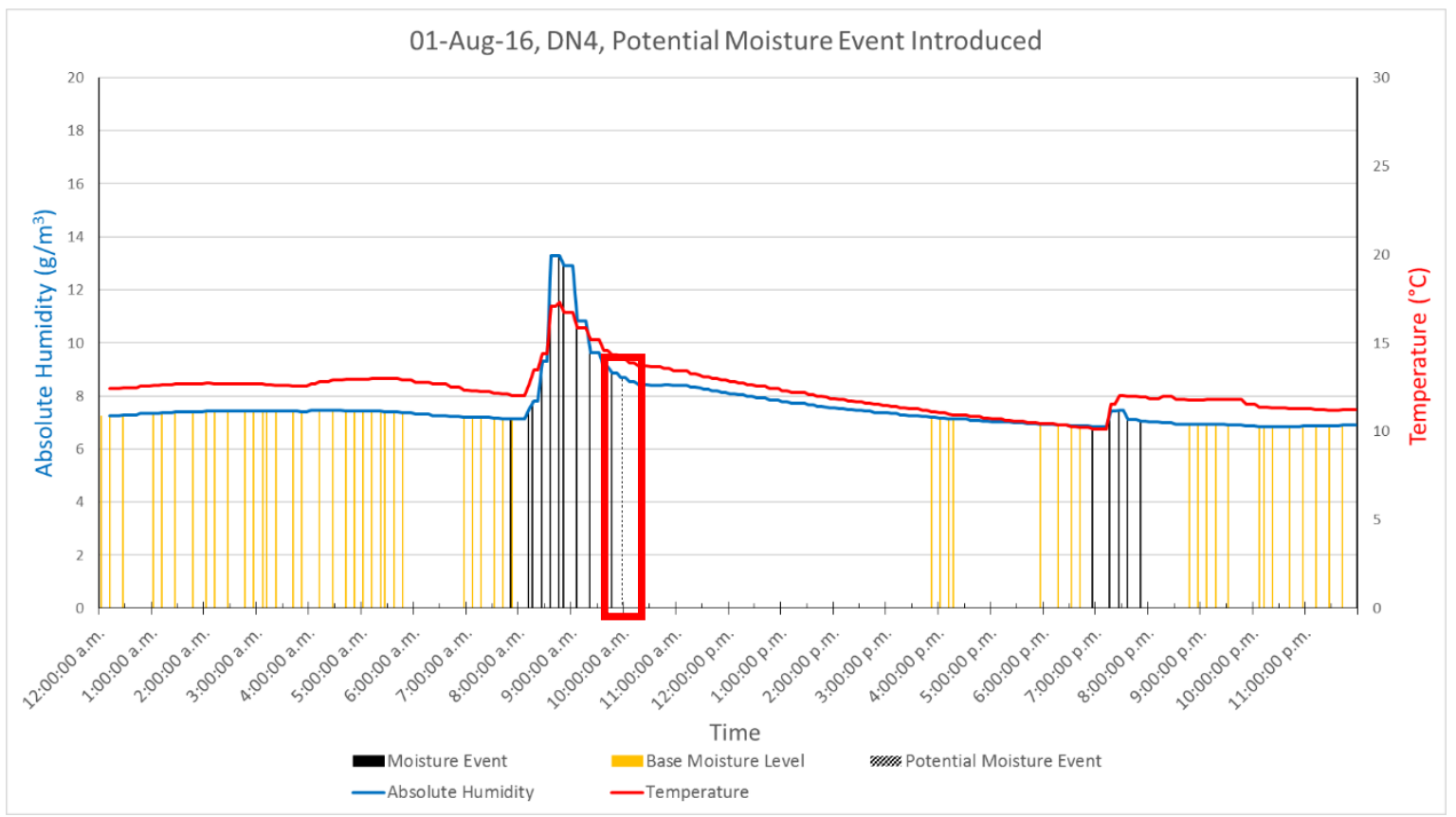

Figure 14 DN4, 'Potential Moisture Event' Introduced at 2\%, Large and Small Event

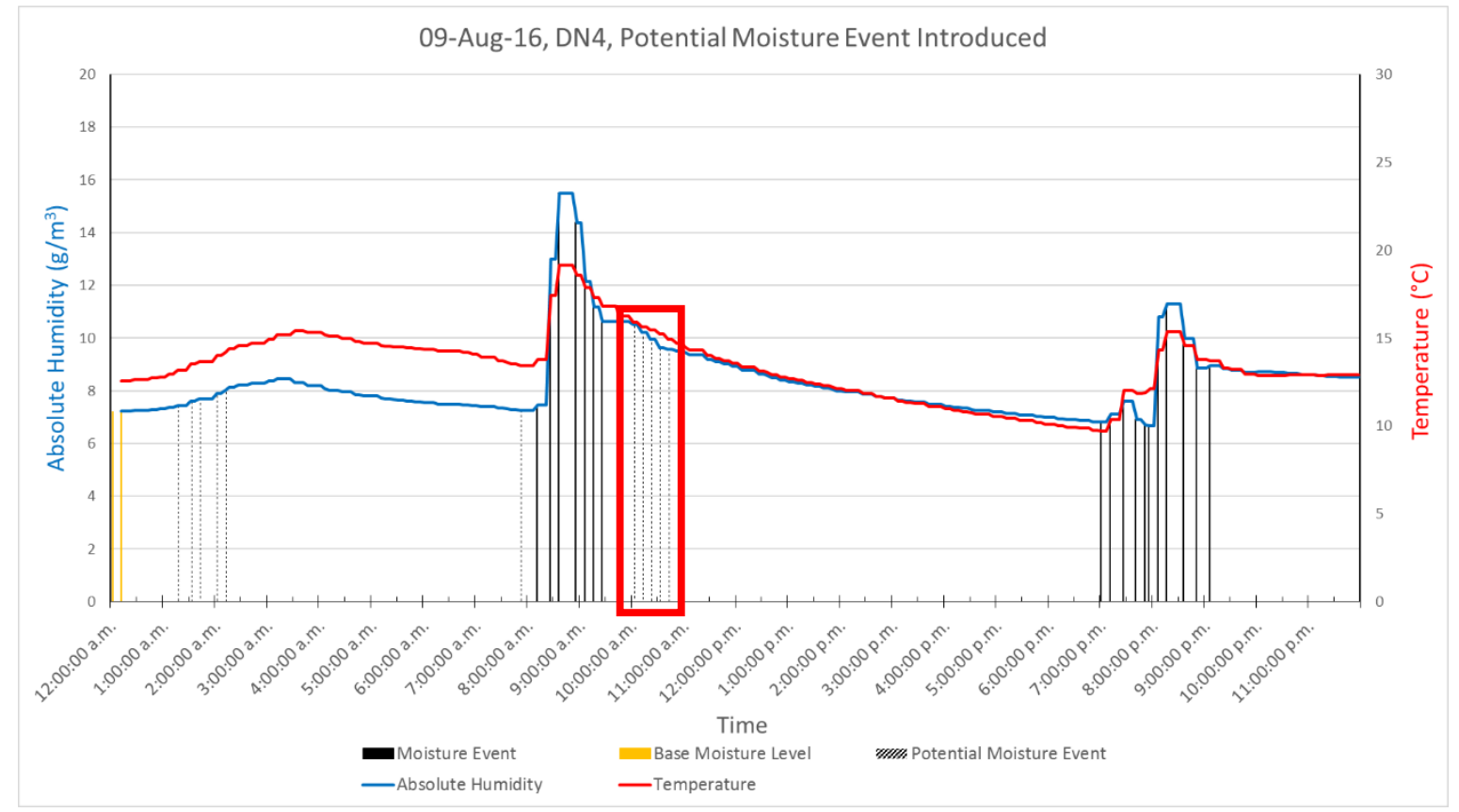

Figure 15 DN4, 'Potential Moisture Event' Introduced at 2\%, Large and Small Event

Figure 14 shows how potential moisture events could be implemented. Moisture events that can be safely assumed to be correct are presented in black, while potential events are presented in a black dashed line. This communicates when the moisture event being analysed is taking place, but also indicates how the indoor environment may have changed before and after the event. 
Figure 15 demonstrates potential moisture events not related to an identified moisture event. This is helpful for understanding how the indoor environment changes over time but, for the most part, can be safely ignored.

\subsubsection{Base Moisture Level}

\subsubsection{1 $\underline{\text { Purpose }}$}

Only considering moisture events for the initial data test would have provided little information on the wider impact they have on the indoor environment. The tests also identified periods experiencing a rate of change below a specified level to prevent this. $A$ period meeting these criteria was described as 'base moisture level' to indicate this was the level of absolute humidity in the indoor environment when no moisture sources were actively introducing moisture. This provided additional information to inform the analysis and to highlight how long it took for the absolute humidity in the indoor environment to reach a stable level after a moisture event.

Base moisture levels do not necessarily identify the only periods where the indoor environment was not influenced by moisture events. It was a method for identifying periods that could be safely assumed not to be influenced by moisture events. Some of the houses did not fall to base moisture level most days. While in some cases this was due to multiple moisture events throughout the day preventing the bathroom from stabilising, other houses experienced naturally large fluctuations in absolute humidity during the day, potentially due to the building design and age.

\subsubsection{Determining Rate of Change}

Unlike moisture events, the base moisture level could not be determined by comparing the difference in absolute humidity between two measurements against the criteria. The low rate of change would need to be maintained for a certain amount of time before being classified as reaching the 'base moisture level'. For example, absolute humidity that stays around $7.50 \mathrm{~g} / \mathrm{m}^{3}$ for five minutes (which may represent two measurements in a row showing the same result) would not count as stabilising. However, once it stayed at this level for 20 minutes (which may represent five measurements in a row showing the same result), it would be classified as having reached 'base moisture level'. 


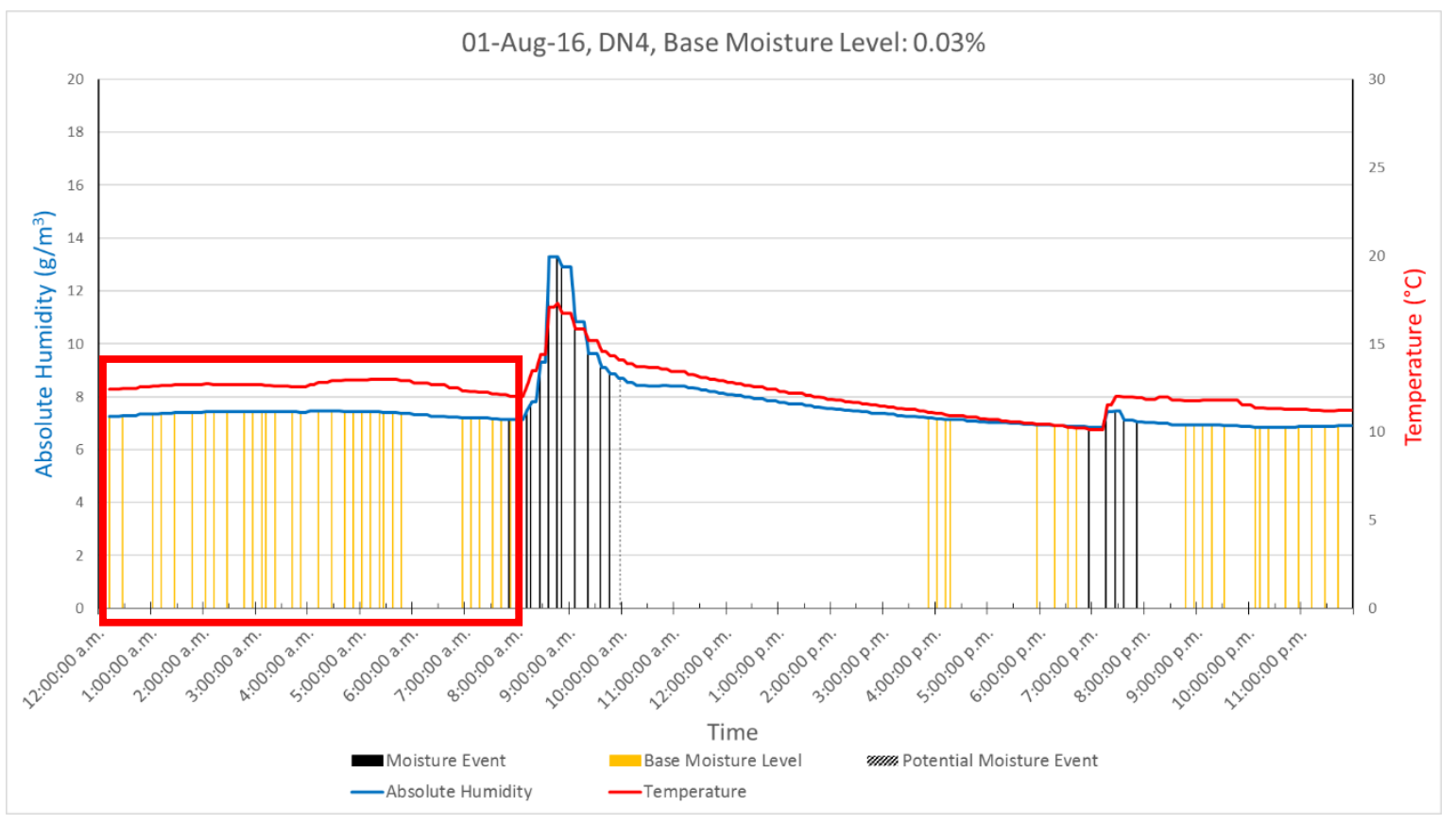

Figure 16 DN4, 'Base Moisture Level' at $0.03 \%$

Figure 16 shows the selected rate of change level: $0.03 \%$. This was found to identify most periods where no noticeable changes in absolute humidity took place while still being large enough to count small changes that took place over several hours. This is based on the same priorities used to identify moisture events, as described in 5.3 Moisture Events. The most important factor for determining base moisture levels is that the rate of change used is consistent for all houses tested.

\subsubsection{Falsely Identified Base Moisture Levels}

This method of determining base moisture levels assumed that for any period where there are no large changes in absolute humidity taking place, that period was not being actively influenced by a moisture event. This was not always the case. In some cases, moisture events or changes in the external environment may have begun before earlier moisture events had ended. If this occurred, there was a possibility there may be a brief overlap with moisture introduced at a similar rate to the moisture being removed. This would result in the absolute humidity appearing to have stabilised, registering as a base moisture level. One potential solution was to introduce a second class of base moisture levels, working in the same way potential moisture events. This would have been based on length rather than a different rate of change. Increasing the length required to be classified as the base moisture level would decrease the chances of any periods still influenced by moisture 
events being identified. This was found to be unnecessary, with most examined base moisture levels appearing to reflect periods that did not appear to be influenced by moisture events.

\subsection{Objective 2: Developing Visual Representation}

A variety of ways to visually represent the data were explored. This section explores some of the different graphs used throughout the original data exploration. The figures have been included to provide insight into how the data could be expressed most clearly, rather than to review the data itself. The goal of this process was to create a graph that would be readable and easy to understand. Note that because of this, many early graphs (Figures 17 to 29) are difficult to read.

\subsubsection{Understanding the Data}

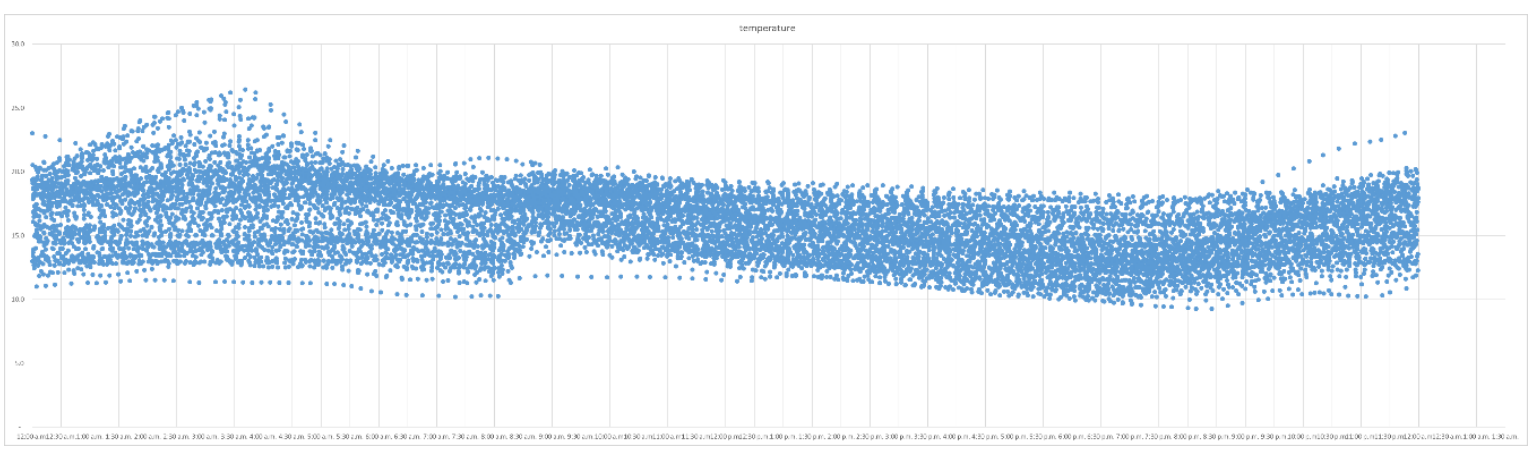

Figure 17 Daily Temperature for July, DN4

The data had to be explored and tested to express the factors most relevant for the analysis. An early example of visualising the measurements is shown in Figure 17. This graph presented measurements of temperature in all rooms. Measurements for the entire month were overlapped to help identify patterns in how temperature varied between days across the house as a whole. 


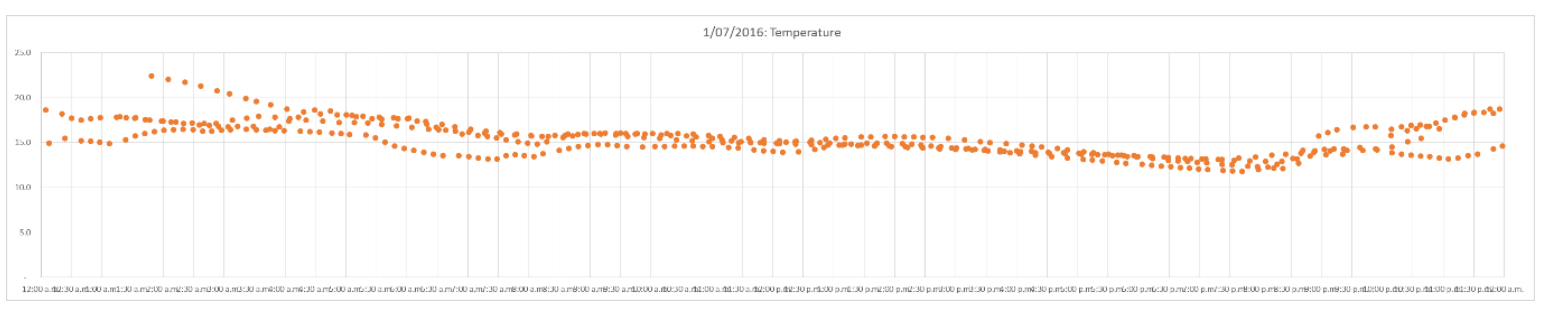

Figure 18 Temperature for 1/07/16, DN4

Days were also reviewed individually, as can be seen in Figure 18. This particular graph shows temperature for a single day in four different rooms. As the data overlaps, this information becomes difficult to read. This was designed as a brief overview of the data, meaning that being able to differentiate between the different rooms was unimportant.

\subsubsection{Presenting Temperature and Humidity}

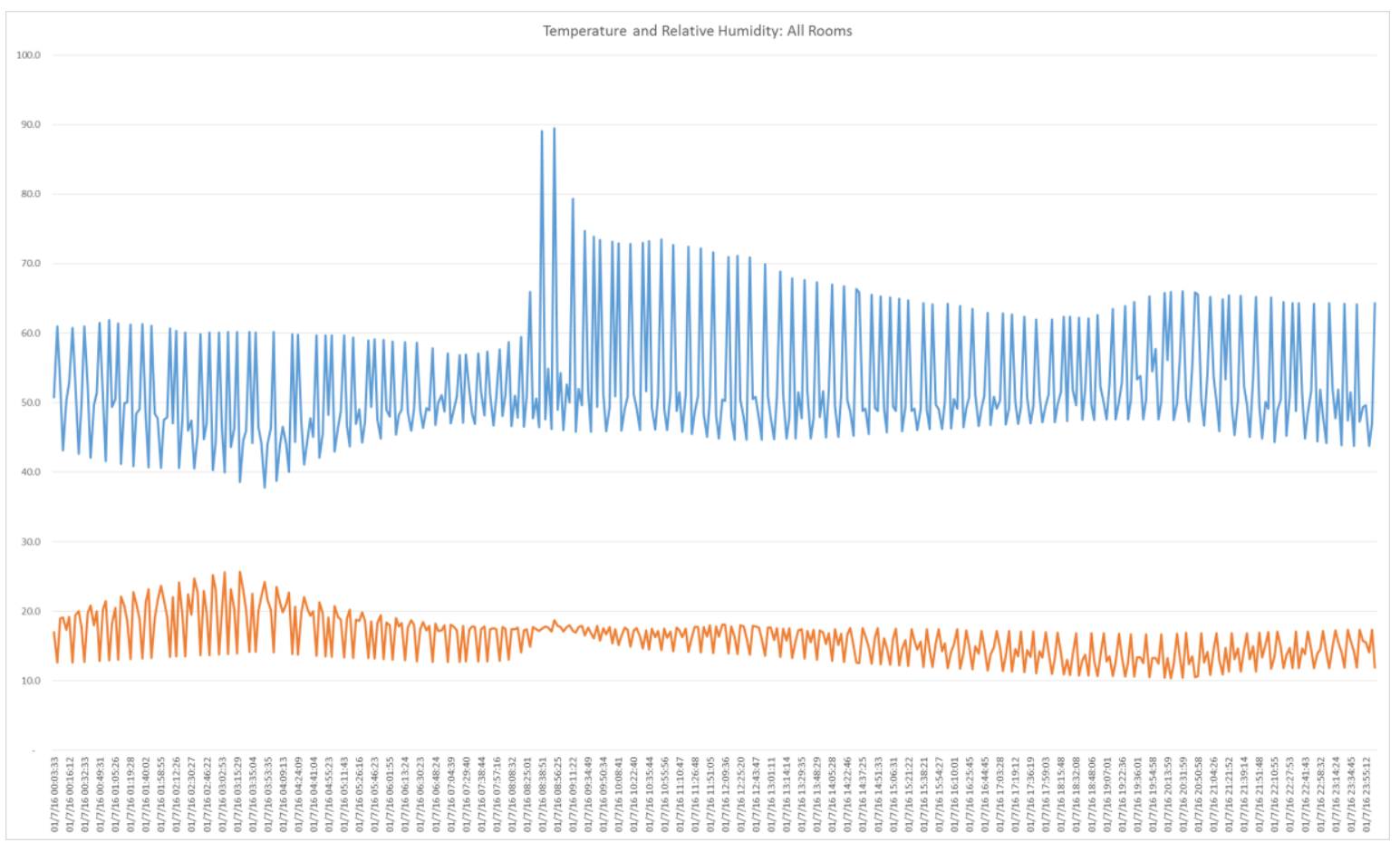

Figure 19 Temperature and Relative Humidity, DN4

The next step was to consider how to best display both pieces of key information: temperature and humidity. This early iteration, Figure 19, places both measurements on the same axis, with relative humidity as a percentage and temperature in degrees Celsius. This makes the graph more difficult to understand, misrepresenting both the type of information presented as well as how the two measurements relate to each other. 
This also makes the temperature more difficult to read, compressing it into a smaller area. Ideally, both measurements should use as much space as required to show any subtle changes in measurements. This type of graph is better suited for showing patterns in the measurements over time.

The limitations of working in Excel resulted in a confusing graph, making it unclear that measurements were taken in four different rooms. The different measurements were shown as a single source when treated as a line graph. This made the line jump between each measurement. While the rooms with the highest and lowest measurements were easy to read, rooms with measurements in the middle were more difficult, with some changes almost impossible to see. Furthermore, connecting them with a line like this disguises the purpose of the graph, making the reader unlikely to compare measurements between different rooms.

\subsubsection{Focusing on Key Details}

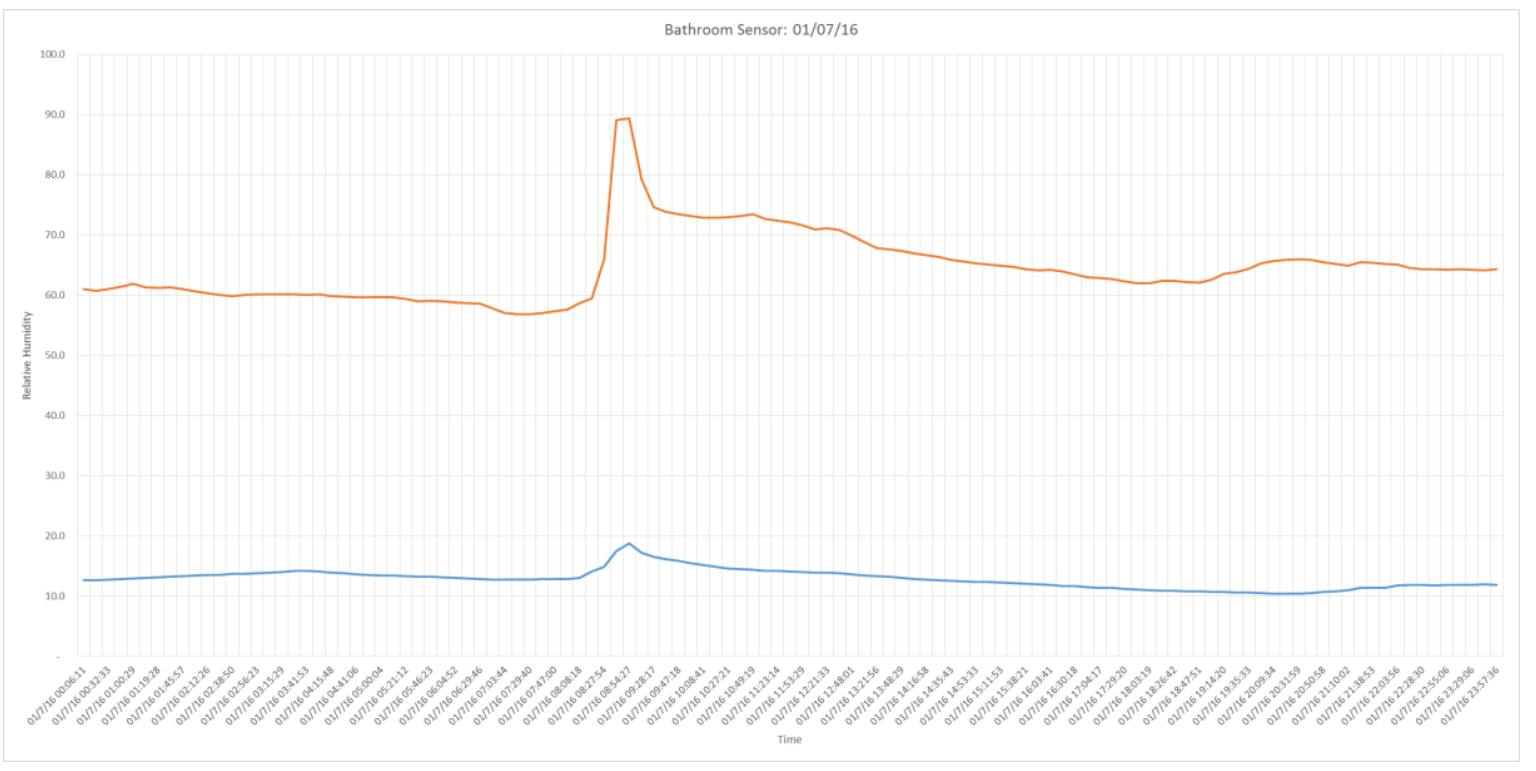

Figure 20 Bathroom Data, DN4

The next iteration, Figure 20, focused on the bathroom. Switching to a line graph made the graph's message far easier to understand, clearly communicating that this was a single variable changing over time. The only downside of this was it no longer indicated the individual measurement points, making it difficult to see how far apart measurements took place. 
Although this graph was an overall improvement, there were still serious issues with how it presented information. Both measurements continued to be measured on the same $y$-axis, making changes in temperature impossible for the reader to accurately review. The graph also fails to highlight what parts of it are the focus of the study. This is made more confusing by the $x$-axis misrepresenting the amount of time between measurements. Each point of measurement was evenly spaced, implying they took place at regular intervals. In reality, a measurement was taken whenever a change was detected. This meant periods with little change, such as during the early hours of the morning, are condensed into a smaller length of the $\mathrm{x}$-axis. By contrast, periods experiencing rapid change have more measurements taken in a short amount of time and are stretched out over a longer length of the x-axis as a consequence. This smooths out the graph, making any sharp spikes appear to be more gradual.

\subsubsection{Comparing Results between Days from a Single House}

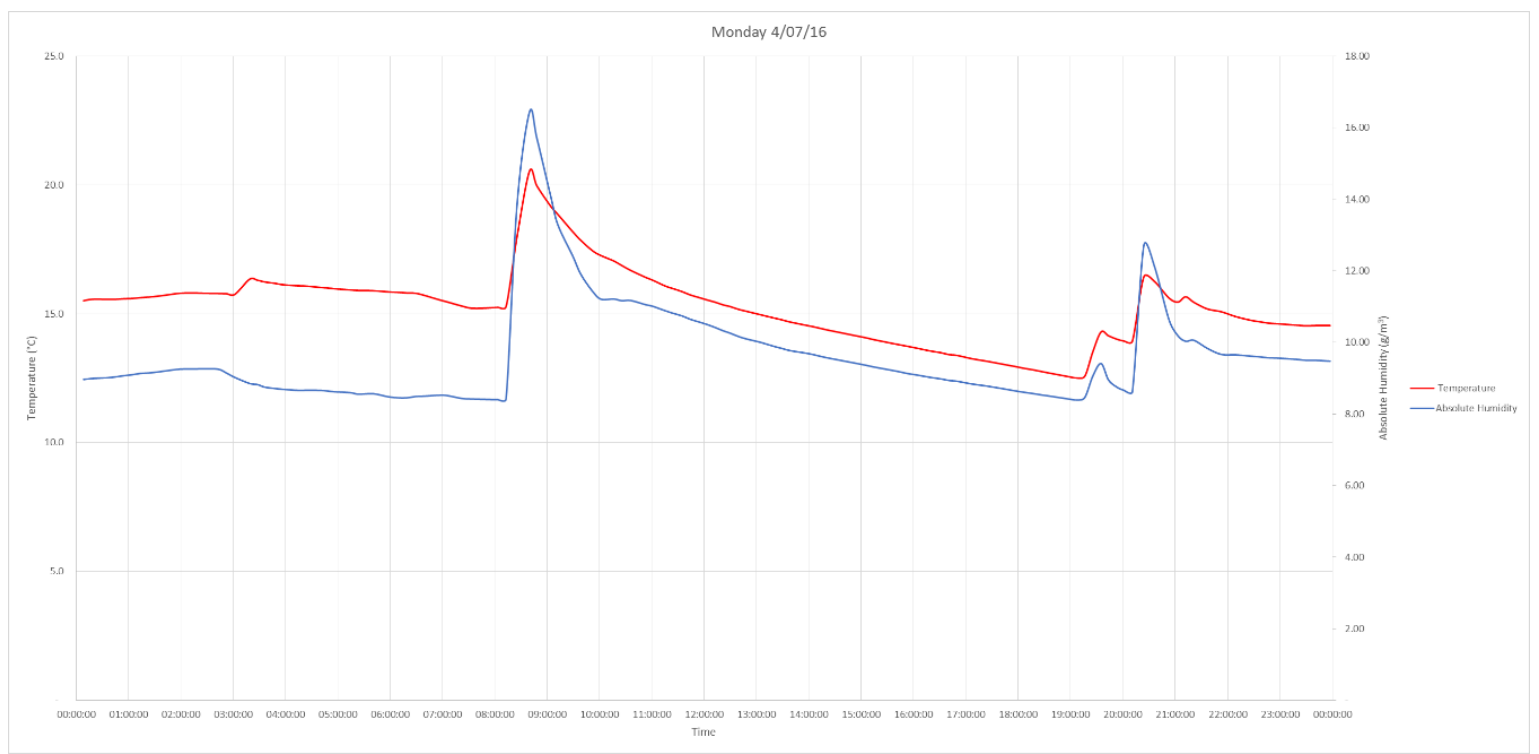

Figure 21 Introducing Absolute Humidity, DN4

Figure 21 built on previous iterations and made several notable improvements. The most significant of these was switching from using relative humidity to absolute humidity. As the moisture was being presented alongside the temperature, it made no sense to use a unit dependant on temperature. This change helps the reader to focus on the graph's purpose: demonstrating what changes are taking place in the bathroom environment. With both measurements independent of each other, when both measurements spike or drop it is 
clear this was caused by changes in the temperature and humidity rather than having to figure out how much of the change in relative humidity was simply caused by changes in temperature.

The two types of measurements were measured over two different y-axes. Temperature, ranging from $0^{\circ} \mathrm{C}$ to $25^{\circ} \mathrm{C}$, was presented on the left axis with the absolute humidity, ranging from $0 \mathrm{~g} / \mathrm{m}^{3}$ to $18 \mathrm{~g} / \mathrm{m}^{3}$ was on the right. This not only made it clear that each line represents a different type of measurement, but also means they can be appropriately sized, making them far easier to read.

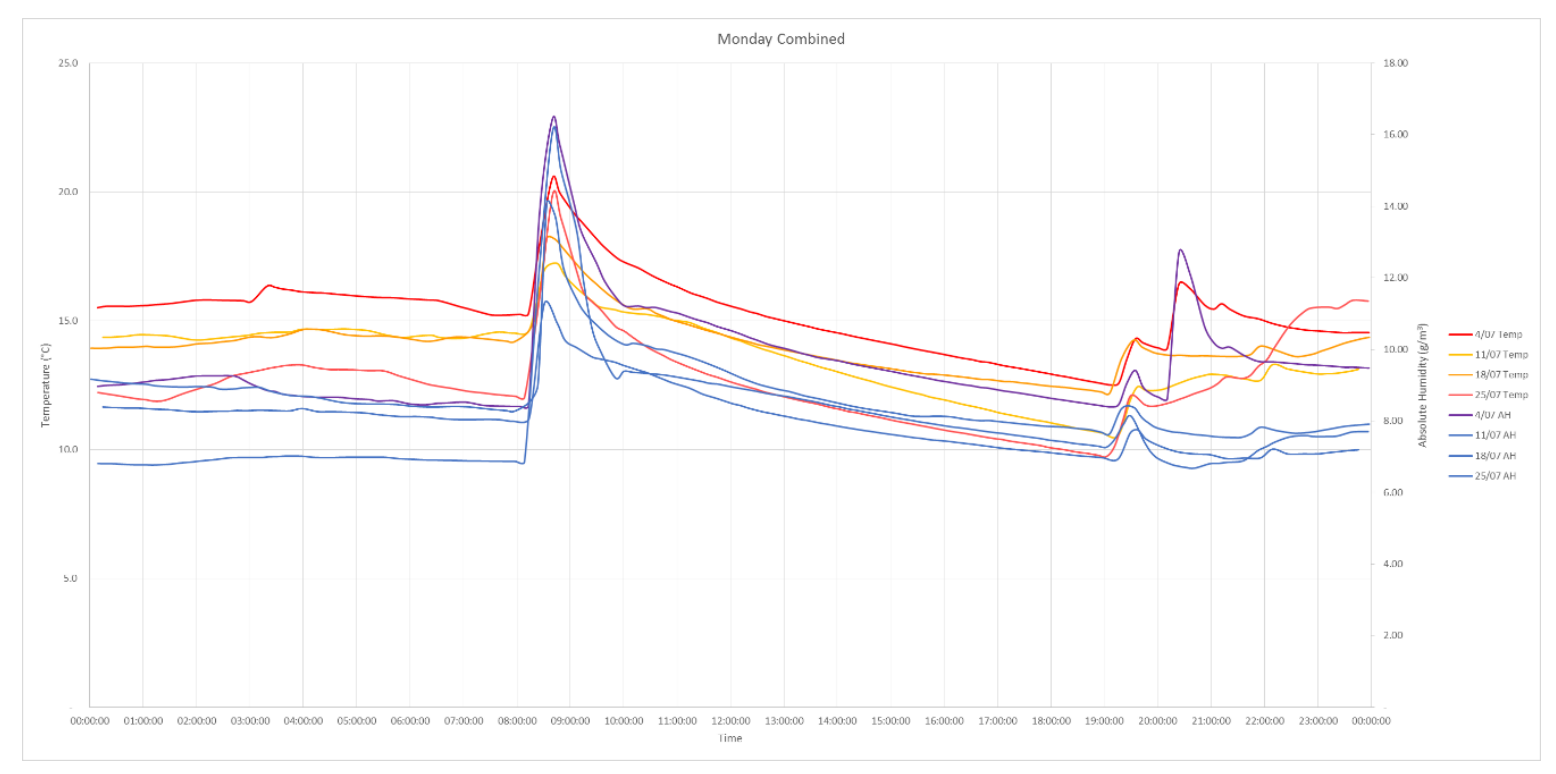

Figure 22 Comparing Days Between Weeks

Another option considered was whether this system may allow data from several days to be overlaid and compared, as shown in Figure 22. Doing this with a line graph made it easier to read, but there was simply too much data presented to do anything more than provide a simplified example of common trends between days. The colour format contributed to this confusion. In an attempt to keep temperature and absolute humidity colours consistent while making each day distinct, many of the lines looked similar. While this type of graph could potentially provide useful information, it would need to be limited to graphs with only one type measurement or a small number of days. 


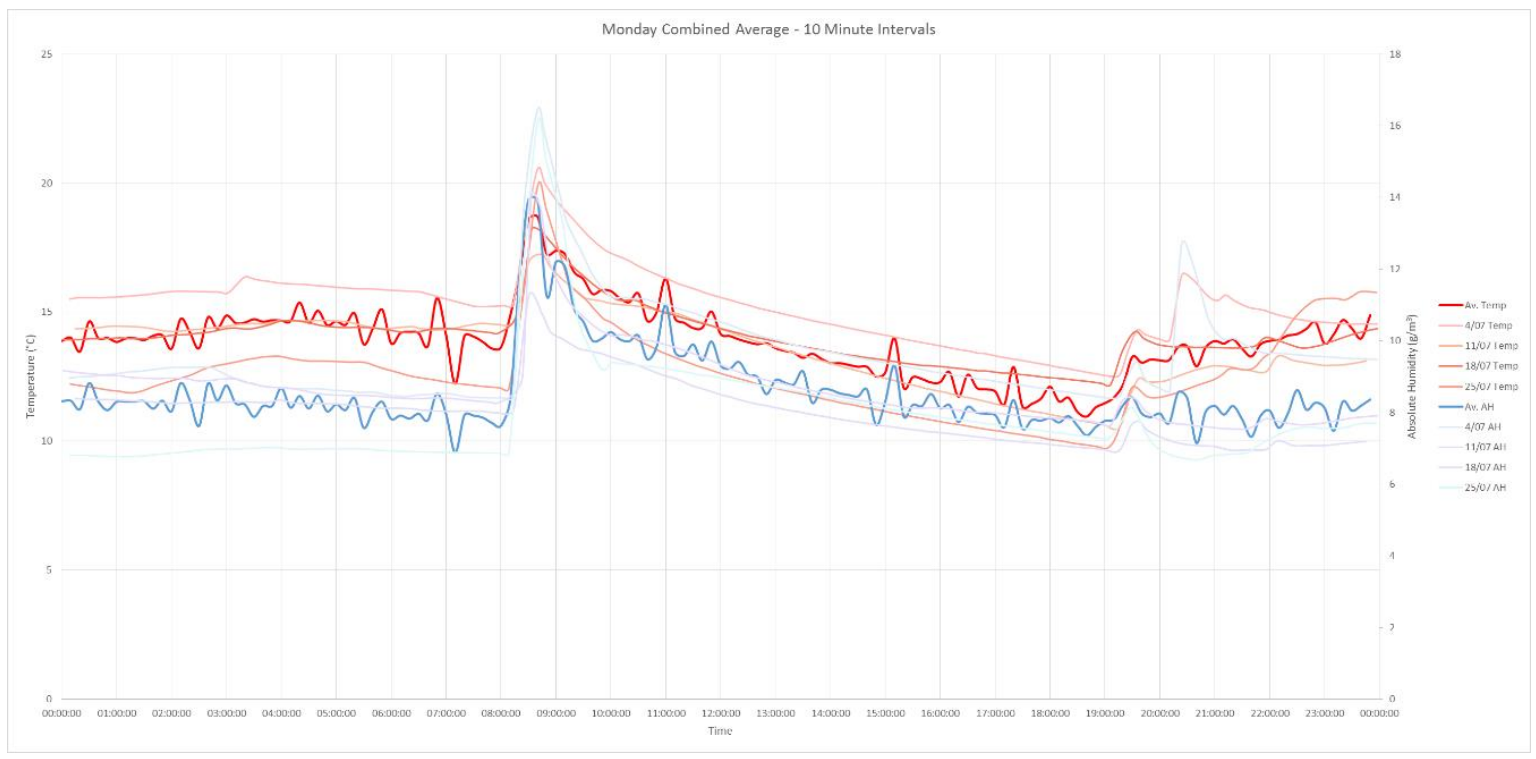

Figure 23 Average Over 10 Minute Intervals

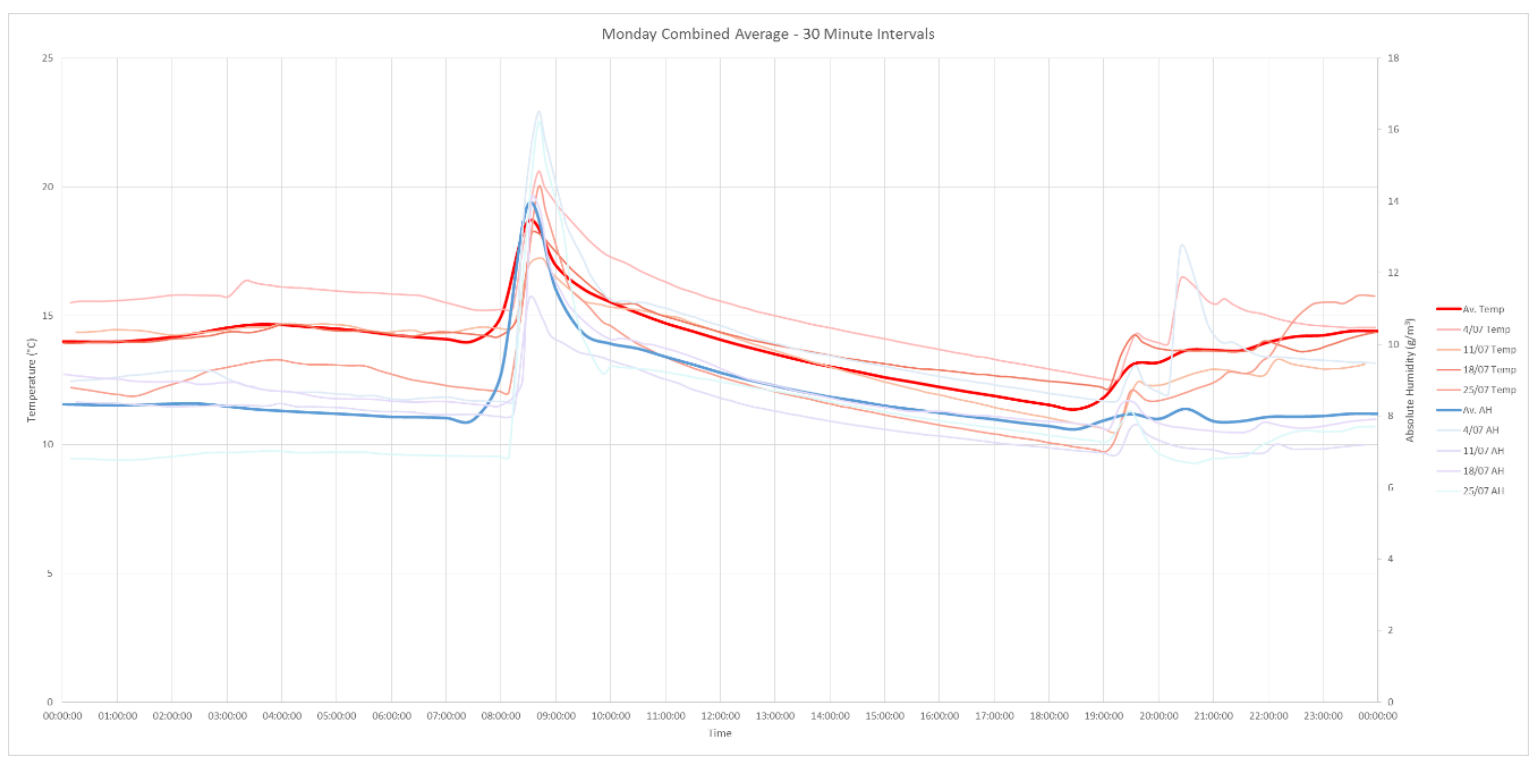

Figure 24 Average Over 30 Minute Intervals

As measurements were taken at irregular intervals, there was no way of directly comparing measurements for any given time. To compensate for this, all measurements from a specified period were combined for each day. The mean for all of these periods could then be listed as the average measurement. The smaller the length of this period, the closer the results would be to the true average. The first attempt at this used periods of 10 minutes (Figure 23). Unfortunately, this meant some days may not have a measurement take place during every 10 minute period. In these cases, the 'average' during some periods would only represent one or two days, causing the line graph to jump between results. 
Using a longer period helped to mitigate this issue. For example, with a period of 30 minutes (Figure 24), it becomes far more likely for every day to have at least one measurement fall within this range. This helped ensure the line graph represents all days at every point. However, this presents a new set of issues. As this average only provides accuracy within a half hour range, it cannot be used for an in-depth analysis of the trends between days. It cannot be used to accurately map the average absolute humidity or temperature during a period of rapid change, such as during a moisture event. 


\subsubsection{Producing Daily Results}

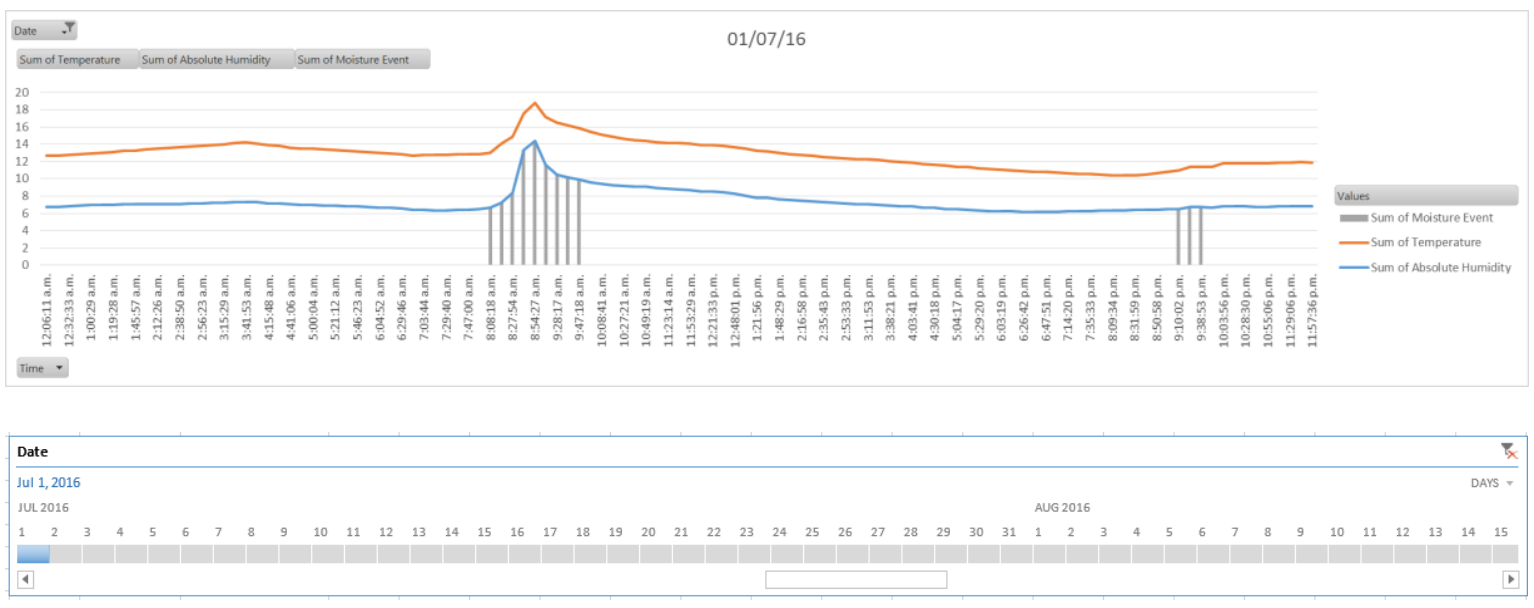

Figure 25 Identifying Moisture Events and Switching Between Days

Figure 25 shows two key improvements: identifying areas of interest and allowing easy comparison between different days without cluttering the graph. A bar graph was introduced to highlight any measurement which registered as a moisture event, as described in 6.3.1 Examples of Different Moisture Event Levels. This not only made it clear which regions of the graph are relevant but also show when each measurement recorded during the event occurred. The absolute humidity line is not cluttered with data and labels, making the graph easier to read as a result.

The graph also began using a filter based on the day measurements were taken. This makes the analysis process far easier, allowing a single graph to switch between different sets of data quickly. This ensured consistency between graphs and provided the means to compare any given days.

One issue the introduction of the bar graph highlighted was the spacing between measurements. As discussed in 6.4.3 Focusing on Key Details, the x-axis presents each measurement as having been taken at regular intervals with the same spacing between each point. This needed to be addressed to accurately represent the rates of change of both temperature and absolute humidity. 


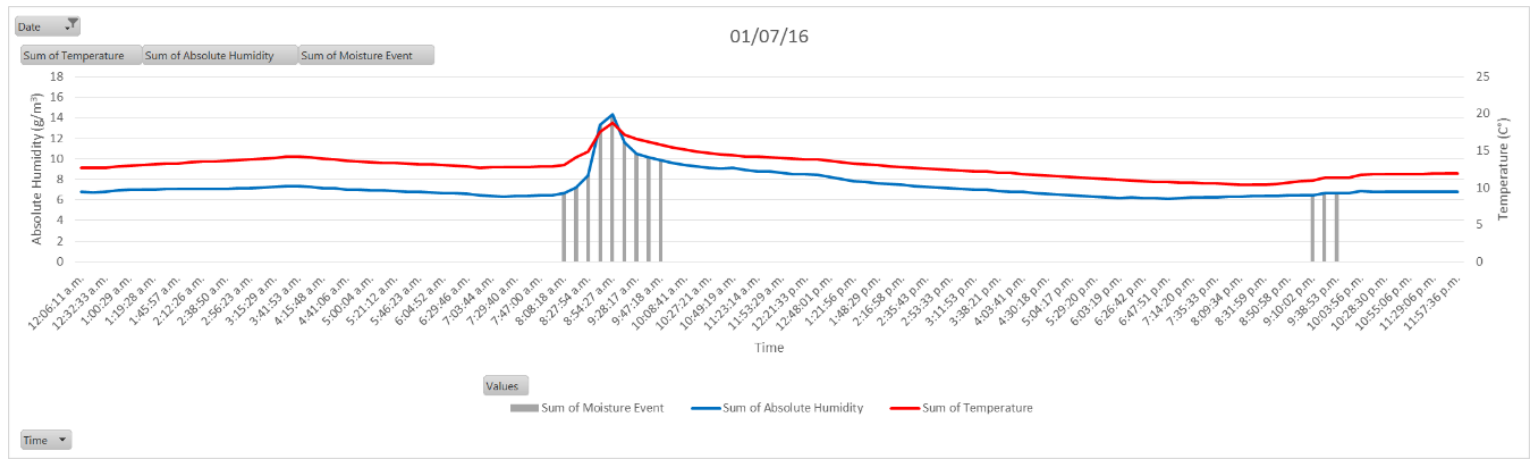

Figure 26 Incorporating Previous Improvements

This was updated to incorporate the improvements made in Figure 21, splitting results over two different vertical axes. However, these two axes were switched from previous iterations, with absolute humidity now displayed on the right. This was done to make the graph more intuitive, with the result more important to the analysis being listed in the main vertical axis (on the left). Whether absolute humidity plays a more important role than temperature when considering the impact of moisture requires more in-depth research that is beyond the scope of this study. However, the focus of this graph is on moisture events, defined by rates of change in moisture events. This justifies treating absolute humidity as being the first measurement the reader should consider when reviewing the graph. The temperature will continue to be included to provide additional information about the state of the indoor environment.

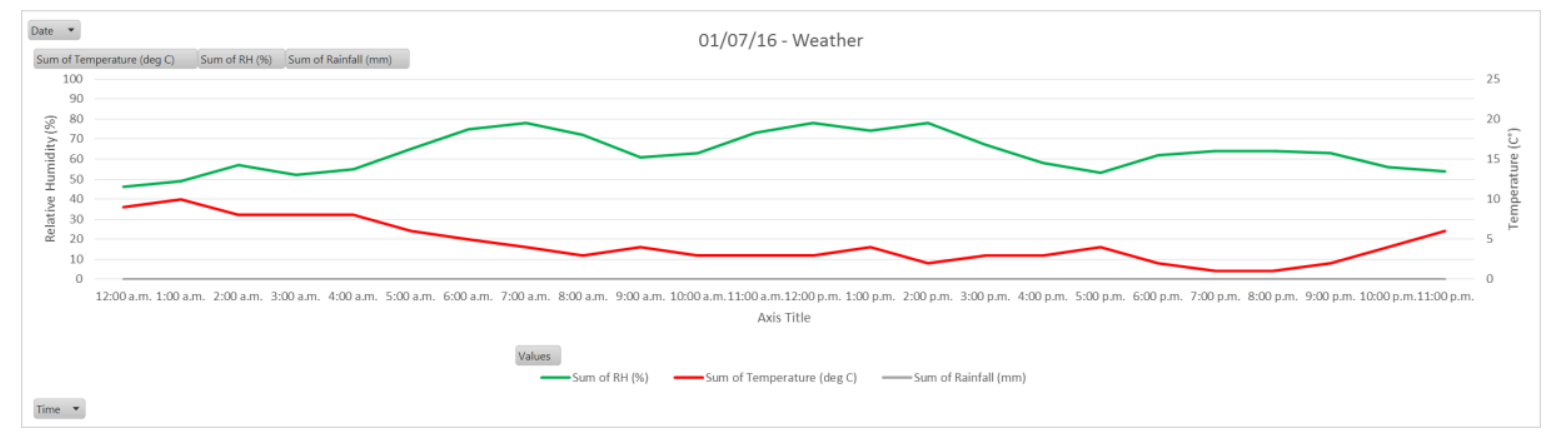

Figure 27 Comparing with Weather

At this point, the information provided by the graph needed to be considered in a wider context. The outside weather used the same type of data. This meant it could be displayed using a similar format. This initial investigation of outside conditions used relative humidity rather than absolute humidity. To differentiate the two, green was chosen to represent relative humidity in the few situations it would be required. 
The temperature axis was left the same. This allowed the inside and outside temperatures to be more easily compared as well as ensuring the temperature was not misrepresented. As a consequence, the outside temperature, which is typically lower than the indoor temperature, takes up a smaller portion of the available space. This made it more difficult to read.

\subsubsection{Accurately Representing Information}

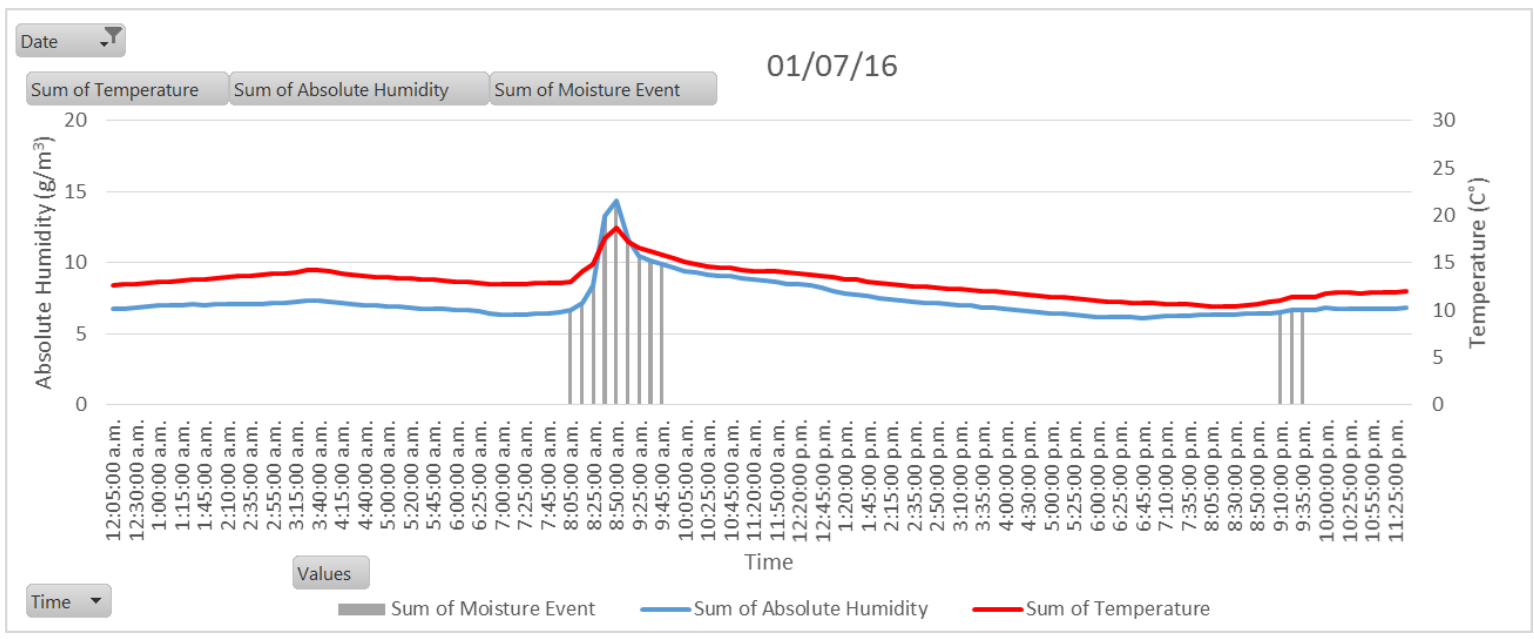

Figure 285 Minute Steps

The next iteration, Figure 28, aimed to fix previous issues with misrepresented lengths of time between each measurement. A standardised length of five minutes was introduced, using the mean measurement of any results taking place within that space of time. This meant the $\mathrm{x}$-axis used increments of five minutes, accurately representing when each measurement occurred. In this particular iteration, the x-axis still appears cluttered and messy, making time difficult to read despite the improvements made.

Both vertical axes were also changed. The absolute humidity now ranged from $0 \mathrm{~g} / \mathrm{m}^{3}$ to $20 \mathrm{~g} / \mathrm{m}^{3}$ while the temperature ranged from $0^{\circ} \mathrm{C}$ to $30^{\circ} \mathrm{C}$. This was to help make individual measurements easier to compare with their respective axis, making the graph easier to read as a result. 


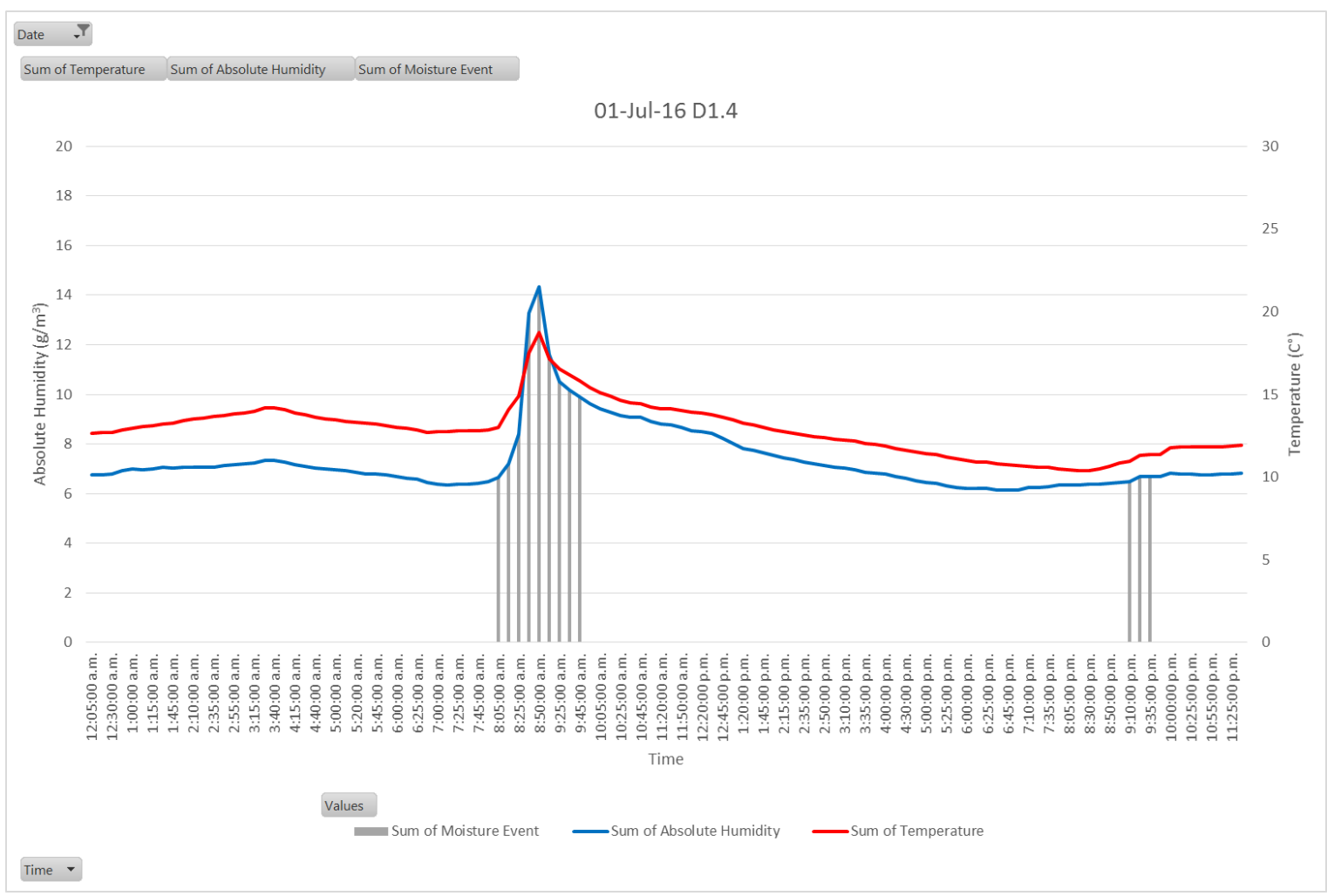

\section{Figure 29 Increased Height}

Increasing the height of the graph helped to make data more readable, making changes in the readings over time more obvious. This had the potential to slightly mislead the reader, making subtle changes in temperature or humidity appear far more significant than they are in reality. However, these subtle changes are important to look at when considering which changes should register as moisture events or be analysed in more detail, so this was an appropriate change. 


\subsubsection{Final Graph}

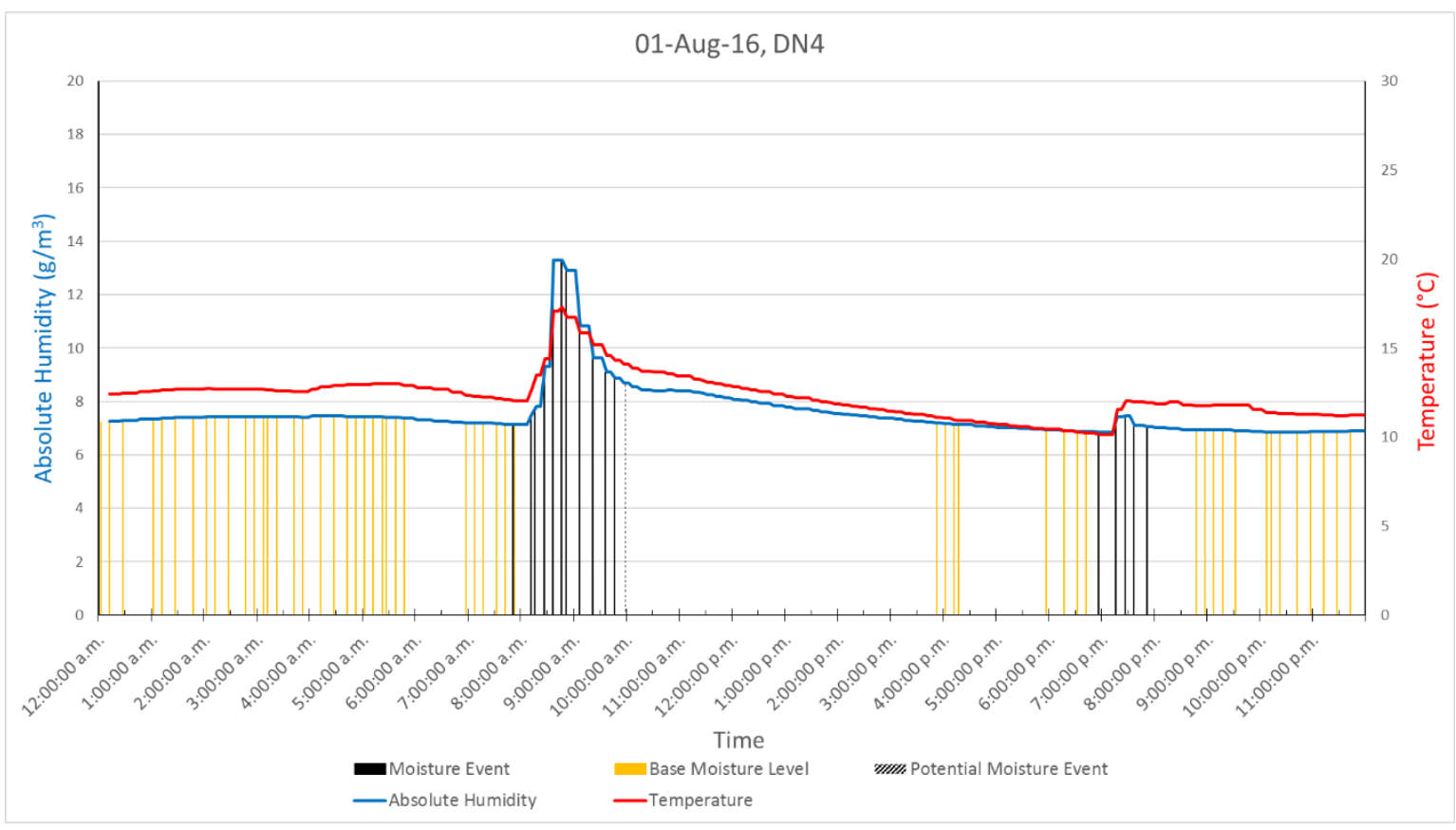

Figure 30 Final Graph

Figure 30 is an example of the fully developed graph. This incorporates all improvements made while testing the data, as well as any other data that would be useful for further analysis. Potential moisture events, as discussed in 6.3.2 Potential Moisture Events, were added to provide additional context to moisture events. These were represented by a black dashed line. This helped to visually communicate their relationship to moisture events, representing measurements which nearly qualified but did not quite reach the necessary rate of change level.

The base moisture level, representing periods of time with very little change, was represented by yellow bars. As discussed in 6.3.3 Base Moisture Level, this represented periods of 20 minutes or more with a rate of change of $0.03 \%$ or less between each measurement. The yellow colour was designed to contrast the black moisture event bars, helping the reader understand these bars represent very different rates of change. Measurements of the absolute humidity rate of change (moisture event, base moisture level, and potential moisture event) are shown on the top of the legend. Measurements of the indoor environment (absolute humidity and temperature) are shown on the bottom. 
The final changes made to the graph design were made to improve readability when printed. The colour of both vertical axes titles was changed to match the colour of the corresponding data, making it clear which axis each line is measured against.

The horizontal axis is measured in hour-long increments with ticks every half hour, making it easier to read. The font size of all text was increased to make it easier to read. A black line was added to represent each axis, making it clearer where exactly the data for that day begins and ends. The line was made a different thickness to the moisture event bars to prevent confusion.

\subsection{Objective 3: Testing Data}

6.5.1 Information about the Selected Example House

This initial investigation focused on only one month of data. This allowed an in-depth investigation to take place within the limited amount of time available. It also meant seasonal changes and other variations due to the time of year could be ignored. This was an issue that also needed to be addressed in the full-scale data analysis.

As with measurements taken in all houses, DN4 had measurements taken when changes in temperature or humidity were detected. This resulted in measurements being taken at intervals typically ranging from five to 20 minutes and allowed changes in temperature and moisture to be tracked over time. Sudden increases or decreases in humidity can be considered a 'moisture event'.

A rate of change of $3 \%$ successfully identified almost all moisture events measured in the house. However, some of the identified moisture events may be false-positives, while other measurements that may qualify as moisture events were not identified. This is a limitation of the data with no perfect solution. There is no way of identifying which moisture events accurately reflect the indoor environment without more information on the bathroom. The best available option is to assume the $3 \%$ rate of change level accurately identifies all events and apply this assumption consistently for the analysis of all houses. 


\subsubsection{Example of Analysis}

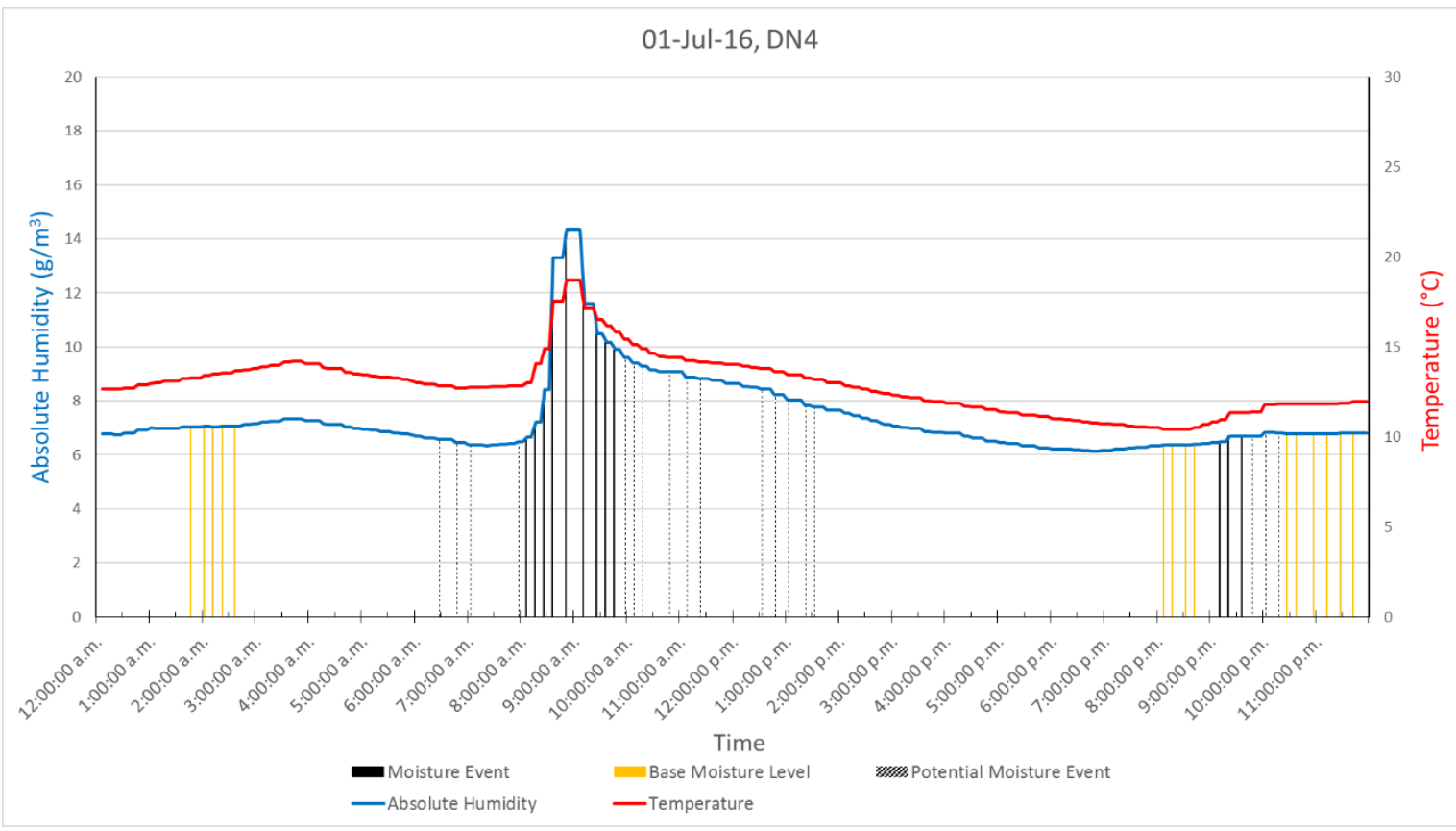

Figure 31 DN4, 1/07/16, Evaluation of Effectiveness

On 1/07/16, shown in Figure 31, the absolute humidity and temperature increase throughout the early morning until 4.00am, then decrease until 7.00am. The rate of change throughout this period at no point it exceeds the $3 \%$ rate of change between measurements, meaning it is not marked as a moisture event.

By contrast, the temperature and absolute humidity rapidly increase from $8.00 \mathrm{am}$ to 9.00am, before decreasing over the next few hours. Only the first hour of this decreasing period reaches a rate of change greater than $3 \%$, meaning everything after $10.00 \mathrm{am}$ does not qualify as being part of the moisture event.

Finally, a moisture event was detected at $9.20 \mathrm{pm}$. This was after the absolute humidity in the room suddenly spiked from $6.48 \mathrm{~g} / \mathrm{m}^{3}$ to $6.69 \mathrm{~g} / \mathrm{m}^{3}$. At $10.03 \mathrm{pm}$ the absolute humidity spiked again, from $6.69 \mathrm{~g} / \mathrm{m}^{3}$ to $6.84 \mathrm{~g} / \mathrm{m}^{3}$. This was only an increase of $2.25 \%$ and did not qualify as a moisture event. Unlike the moisture event earlier in the day, the absolute humidity at this time increased in steps, suddenly rising, remaining at this new level without changing, and then suddenly rising again. This suggests that, rather than being caused by a constant moisture source such as a shower, this was caused by either brief moisture events 
such as taps being turned on or events elsewhere in the house affecting the bathroom in brief bursts such as when the door is opened.

This highlights a variety of situations where using a simple rate of change calculation may not necessarily be the best method of determining whether a moisture event was taking place. The early morning rise and fall show a potential moisture event that may have been missed, but in this case, the gradual change taking place over 8 hours in the middle of the night suggests this was not caused by a moisture source such as a shower. It was likely caused by factors such as the weather or HVAC systems in the house. This is an example of the moisture event criteria of $3 \%$ working as intended, excluding moisture events that take place too slowly to be caused by moisture sources found in typical homes. However, the impact of weather and occupant behaviour must be taken into consideration before entirely excluding the possibility of it being a moisture event.

The moisture event ending at 10.00am while the potential moisture event continues until 11.30am are an example of a situation where the impact of the moisture event is being felt but has been reduced to a point where the absolute humidity is no longer rapidly changing. Unlike the early morning moisture event, this is a situation where a moisture event has taken place, but the point where it no longer classifies as a moisture event is decided somewhat arbitrarily. Stating that as soon as the absolute humidity is decreasing at a lower rate than the predetermined rate of change criteria fails to take into account the many factors influencing moisture events as well as the effectiveness of different systems used to deal with them. The inclusion of the potential moisture event helps to address this issue while consistent moisture event criteria allow for more accurate comparisons to be made between houses.

\subsubsection{Processing Data}

The information presented in the previous section was made by converting the BRANZ data into tables sorting temperature and absolute humidity. This was done by creating a data analysis template where measurements from each house could be imported. This included data ID, temperature, date, time, and relative humidity. A series of steps were performed to process the data before being presented. 
The first step was to calculate the absolute humidity using the temperature and relative humidity. This can be done using a combination of simple equations:

- $\quad P_{w s}=A\left(10^{(m \times T) /\left(T_{n}+T\right)}\right)$

- $P_{w}=P_{w s}\left(\frac{R H}{100}\right)$

- $A H=C\left(\frac{P_{w} \times 100}{T_{n}+T}\right)$

('Humidity Conversion Formulas: Calculation Formulas for Humidity', 2013)

Where:

- $P_{w s}$ is the saturation vapour pressure $(\mathrm{hPa})$;

- $A$ is the vapour pressure proportionality factor (constant);

- $m$ is the molecular weight (constant);

- $T$ is the temperature $\left({ }^{\circ} \mathrm{C}\right)$;

- $T_{n}$ is the triple point temperature (constant) (K);

- $P_{w}$ is the vapour pressure $(\mathrm{Pa})$;

- $R H$ is the relative humidity (\%);

- $A H$ is the absolute humidity $\left(\mathrm{g} / \mathrm{m}^{3}\right)$; and

- $C$ is the specific heat capacity (gK/J).

This assumes the temperatures in each measured space will typically be between $-20^{\circ} \mathrm{C}$ and $50^{\circ} \mathrm{C}$

This conversion was carried out for every recorded measurement. As indicated by the order these formulae are listed in, the $P_{w s}$ was calculated first and was then used to calculate the $P_{w}$. This was then used to calculate the absolute humidity. For each measurement, the previous absolute humidity (from the last measurement) is subtracted from the current measurement. This difference is recorded as the 'absolute change'.

The percentage change between these values is then recorded. If this value is greater (as either a positive or negative percentage change) than $3 \%$, then it is categorised as a moisture event. A final check is then made on measurements not classified as events as to whether measurements directly before or after were classified as events. If this is the case, then the measurement is recorded as being part of that same moisture event. 
All days from the period the house was measured over were broken into five minute periods. This step allows measurements taken at irregular intervals to be accurately depicted when plotted on a graph. A check was run on whether any measurements were taken during each of these periods for each day. This checks against both the date and time, ensuring all measurements are accurately presented. If more than one measurement was taken during one of the five minute periods, then the mean measurement was calculated. The 'blank' periods with no measurements were then shown as being equal to the previous measurement taken. This prevents the problem seen in Figure 32.

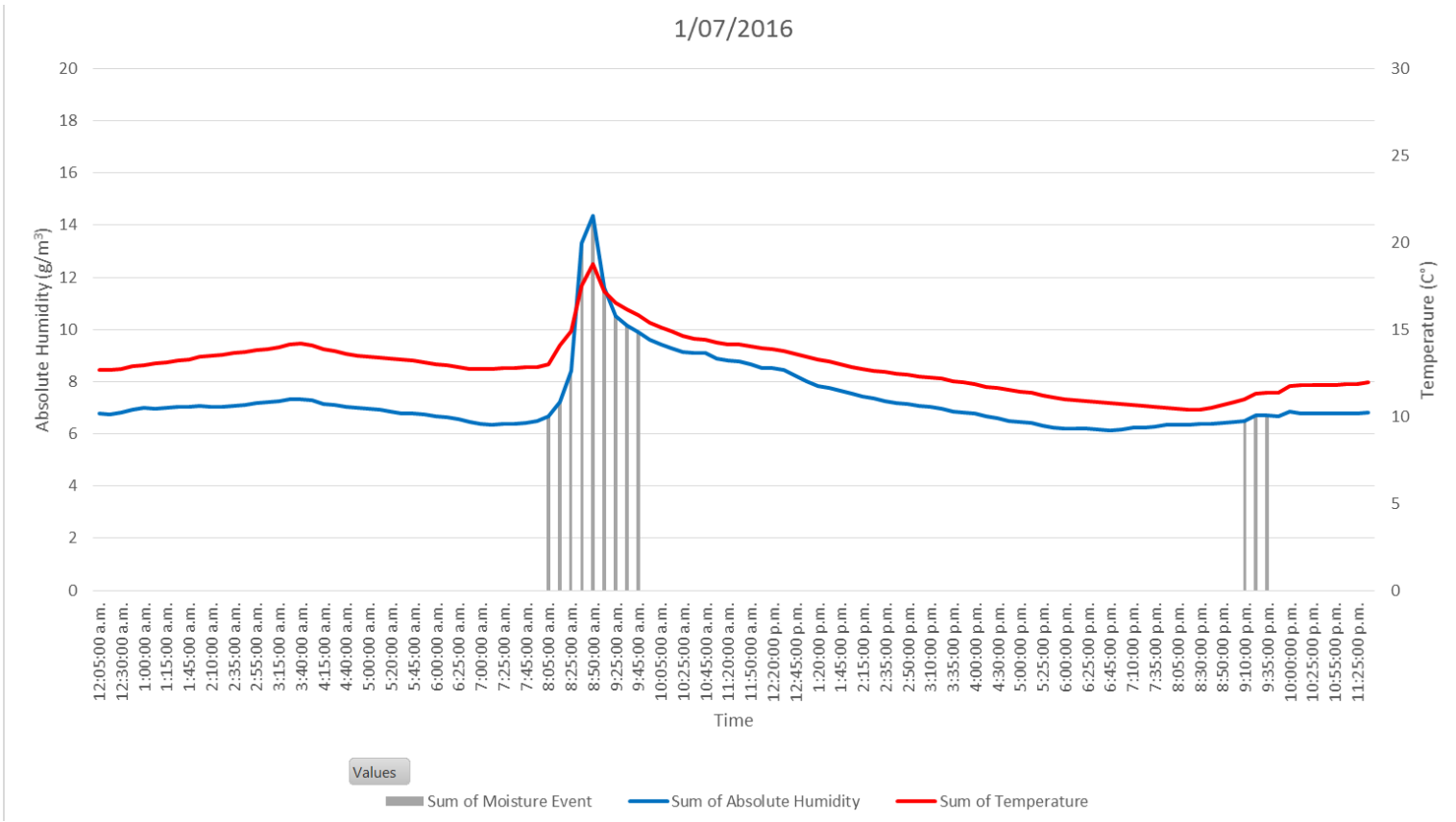

Figure 32 DN4, Early Iteration, 'Sliced' Layout

Figure 32 was created by making these blank periods equal 0 excluding these values. This meant the only points that appear on the graph are ones based on actual measurements. It also results in a smooth line, showing how temperature and absolute humidity change. However, combined with the bar graph showing when moisture events occur, this plots all measurements as if they were taken at regular intervals. With blank periods excluded, this results in an $\mathrm{x}$-axis that misrepresents time. Series of measurements which stayed at similar levels of temperature and humidity for long periods of time have several blank five minute periods, meaning this method will make those periods appear to have taken place over a shorter amount of time. Meanwhile, periods of rapid change have very few blank periods, making them appear to have taken longer. This means that, despite appearing to have more 
natural increases and decreases in measurements, the $x$-axis misrepresents the amount of time this took place over.

Making each blank period equal to the most recent measurement prevents this problem, as can be seen in Figure 31. This works because of how measurements were recorded, with new readings taken whenever a change is detected. This makes it safe to assume that during any blank five minute period following a measurement, little change took place in temperature and humidity. These blank periods can, therefore, be displayed as having the same temperature and absolute humidity as the last recorded measurement. This results in the information being portrayed as taking place in 'steps' (Figure 31) rather than smooth lines.

The main advantage is this prevents blank periods from being excluded, allowing the $x$-axis values to reflect a full day accurately without skipping any time. This accurately portrays the length of time the room stayed at the same levels of temperature and humidity. Periods without change are just as important for analysis as periods with rapid change. The disadvantage is that in almost all cases, the 'steps' presented in the graph do not reflect the rate at which temperature and absolute humidity changed, showing them as sudden spikes rather than a steady increase taking place over several minutes. While this does not matter for analysing the data, as the rate of change is calculated before the measurements are broken into five minute periods, it does make understanding the graphs slightly less visually intuitive.

\subsubsection{Creating a Data Analysis Template}

The method for processing data described in 6.5.3 Processing Data formed the basis of the data analysis template. This template allowed data from any house to be imported, running the calculations on all measurements, and ensuring all results and graphs were consistent. Information about moisture events could then be taken from each graph and compared. 


\subsection{Example of Data Exploration}

\subsubsection{Comparing Moisture Events}

To accurately analyse how effectively moisture is controlled over an extended period of time, common patterns of moisture events would need to be recorded. Days sharing similar patterns which occurred frequently could be counted as being a 'typical day'. Each typical day would take into account patterns in moisture events. For example, if a house has a moisture event beginning at 7.00am every weekday and a moisture event beginning at 9.00am on Saturday and Sunday, then two different typical days will need to be constructed: one for weekdays and one for weekends. Other differences may happen at irregular intervals. For example, if the house appears to be unoccupied on frequent, yet unpredictable, days then this will also require two different typical days. In this case, the frequency of the unoccupied periods will need to be analysed. If on average the house is unoccupied once every ten days then any results produced from the house would be made under the assumption the house will behave in this way $10 \%$ of the time.

This process would be repeated until an example of each type of typical day, as well how frequently they occur, is completed. The average length of time and rate of change between each main point in the moisture event (potential event start, event start, event peak, event end, potential event end, and base moisture level) will then be calculated for each typical day. This will show how the house reacts to moisture in typical situations and allows its performance to be reviewed.

This may mean during weekdays the house will take several hours before the rate of change drops low enough to no longer register as a potential event, returning to a stable level of absolute humidity. By contrast, the weekends may show the amount of time between the event peak and event end is greater on average, suggesting active ventilation systems were left on for longer. Unoccupied days are likely to have no moisture events, and on average may show the humidity dropping during the day, resulting in $10 \%$ of typical days starting with a lower base moisture level.

\subsubsection{Comparison to Weather}

An example of a typical day (1/07/16) is shown in Figure 31, 0 
Example of Analysis. Key changes in absolute humidity include:

- an increase around 8.00am;

- a peak just before 9.00am;

- a sharp decrease for 15 minutes, dropping from $14 \mathrm{~g} / \mathrm{m}^{3}$ to $11 \mathrm{~g} / \mathrm{m}^{3}$;

- a subsequent lower rate of decrease, taking 40 minutes to drop $2 \mathrm{~g} / \mathrm{m}^{3}$; and

- a continuous slow decrease over the course of the day, reaching a low point of just over $6 \mathrm{~g} / \mathrm{m}^{3}$ at $6.45 \mathrm{pm}$.

This pattern suggests the bathroom was not being used during the day after the moisture event at $8.00 \mathrm{am}$. This is further supported by this being a weekday, possibly making it less likely for occupants to be present during the day. This can then be compared to the outside temperature and absolute humidity at the time to understand better the influence these conditions have on the moisture events.

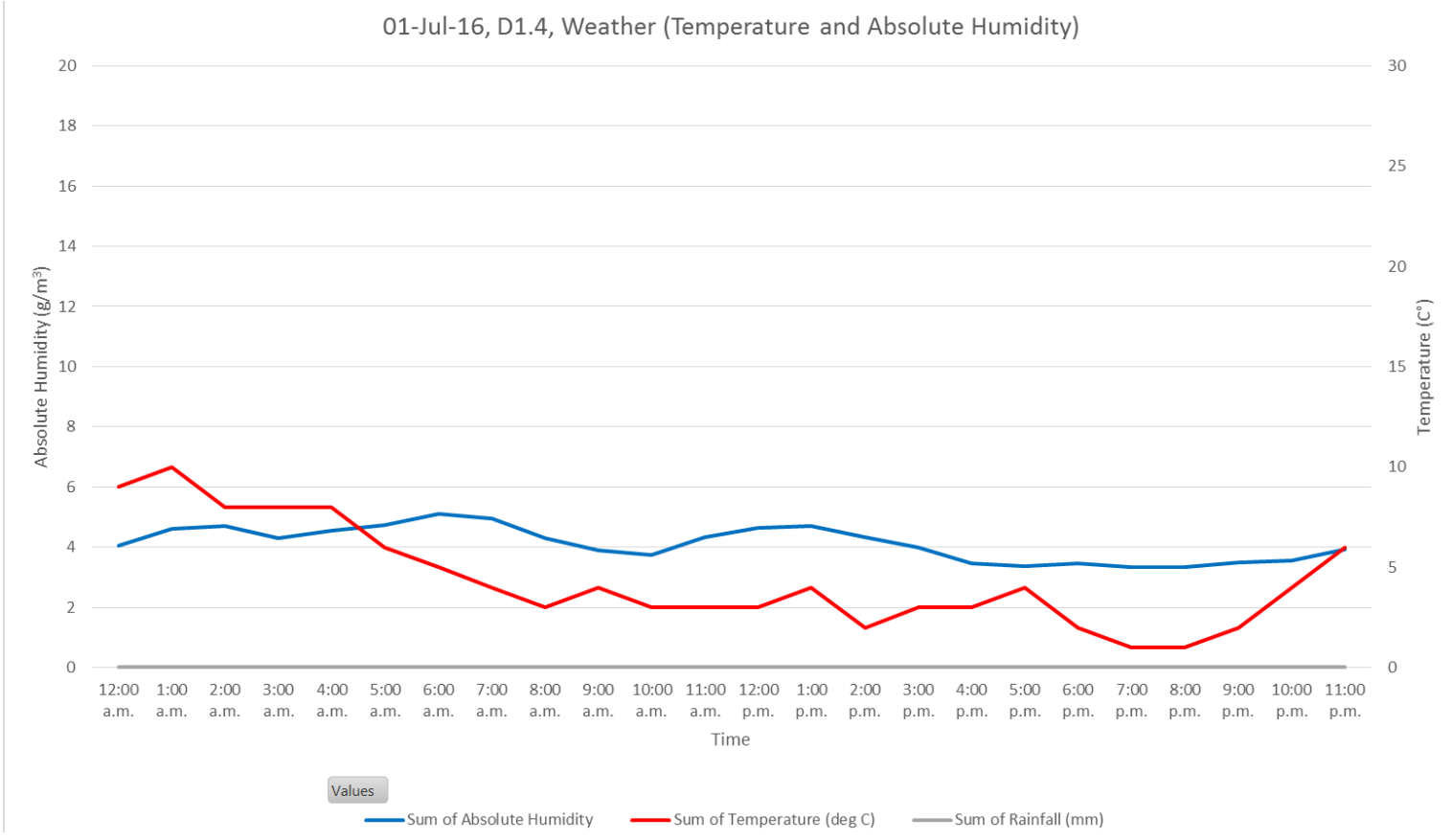

Figure 33 DN4, 01/07/16 - Weather (Temperature and Absolute Humidity)

These changes in absolute humidity in the bathroom occur independently of the outside weather at the time (Figure 33), with the indoor temperature and humidity experiencing little changes during the periods the outdoor relative humidity hit its peaks. This was the case at 7.00am, $12.00 \mathrm{pm}$, and $2.00 \mathrm{pm}$. Relative humidity in Dunedin reached $75 \%$ at $7.00 \mathrm{am}$ before dropping to $61 \%$ at $9.00 \mathrm{am}$. The outdoor absolute humidity, which can be more 
easily compared to the amount of moisture introduced by moisture sources in the bathroom, peaked at $6.00 \mathrm{am}$ with $5.10 \mathrm{~g} / \mathrm{m}^{3}$ and remained at a similar level until 7.00am. By 9.00am, when the indoor absolute humidity had reached its highest point, the absolute humidity had dropped to $3.88 \mathrm{~g} / \mathrm{m}^{3}$.

This suggests the increase of moisture in the bathroom at 9.00am on this day was more heavily influenced by the indoor moisture sources and the ventilation systems in use.

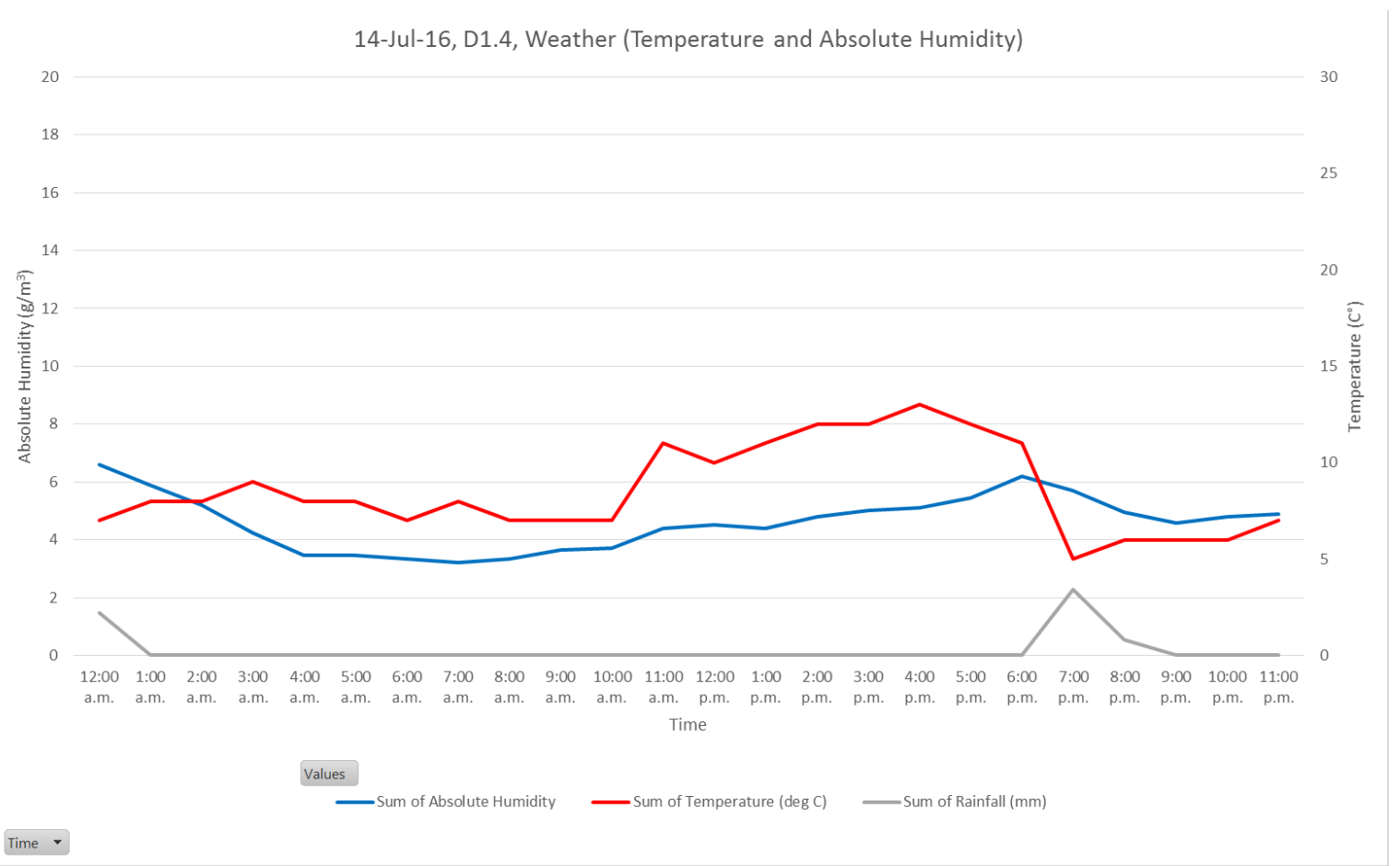

Figure 34 DN4, 14/07/16 - Weather (Temperature and Absolute Humidity)

Unlike the outdoor absolute humidity, days with rain appear to have had an impact on the indoor absolute humidity. At 7.00pm on the $14^{\text {th }}$ of July $2016,3.4 \mathrm{~mm}$ of rain fell in Dunedin (Figure 34). The temperature at the time dropped from $11^{\circ} \mathrm{C}$ to $5^{\circ} \mathrm{C}$, while the relative humidity spiked from $62 \%$ to $84 \%$. The rain then decreased to $0.8 \mathrm{~mm}$ from $8.00 \mathrm{pm}$ to $9.00 \mathrm{pm}$. The absolute humidity peaked at $6.00 \mathrm{pm}$ at $6.21 \mathrm{~g} / \mathrm{m}^{3}$, just before the rain began. 


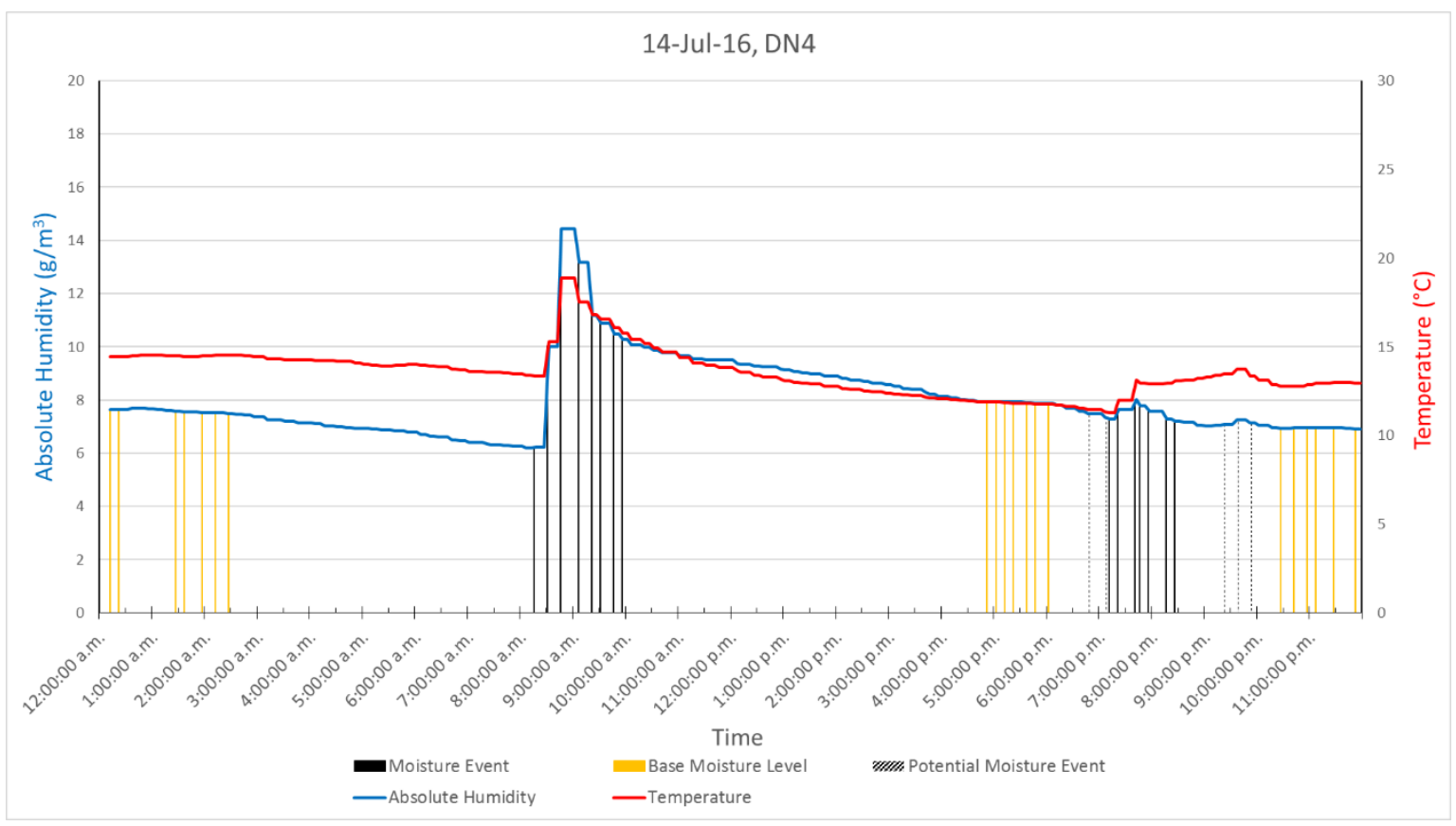

Figure 35 DN4, 14/07/16 - Thursday

The indoor temperature and absolute humidity also spike in the evening, reaching their peak before $8.00 \mathrm{pm}$ (Figure 35). The indoor temperature and absolute humidity for this time do not match the outdoor measurements which were increasing to the highest point for the day as the indoor measurements were decreasing. This can be seen by comparing the period from $12.00 \mathrm{am}-5.00 \mathrm{pm}$. The temperature outside increased from $10^{\circ} \mathrm{C}$ to $14^{\circ} \mathrm{C}$ while the temperature inside decreased from $14^{\circ} \mathrm{C}$ to $12^{\circ}$. At this point, the only apparent influence on the indoor conditions was the remaining effect of the moisture event that had taken place earlier that morning. While it is likely the outdoor conditions affected the rate the indoor temperature decreased, the steady rate of change indicates the moisture event had a far greater impact.

The temperature inside continues to climb until $9.00 \mathrm{pm}$, independently of the absolute humidity. This suggests that rather than the temperature being increased by a shower or similar moisture event, a heater was in use at the time. This was likely positioned elsewhere in the house, with the heat spreading into the bathroom. This could suggest that as the rain started and the outdoor temperature began to fall, heaters were turned on inside, resulting in both temperature and absolute humidity increasing. More likely, an occupant has a shower between 7.10pm and 7.40pm, causing a moisture event and an increase in temperature. The house likely had heaters running during this time, meaning even after the shower the temperature continued to slowly rise until $9.30 \mathrm{pm}$ when the occupants likely 
started getting ready for bed and turned the heaters off. This makes it difficult to know whether the increase in outdoor humidity was having an impact on the indoor environment. The next recorded rainfall in the area took place on the $17^{\text {th }}$ of July, three days later. A small amount of rain fell after midnight, with $0.2 \mathrm{~mm}$ from $12.00 \mathrm{am}$ to $1.00 \mathrm{am}$, and $0.4 \mathrm{~mm}$ from $1.00 \mathrm{am}$ to $2.00 \mathrm{am}$. Most of the rain fell between $3.00 \mathrm{am}$ and $4.00 \mathrm{am}$, with $3.4 \mathrm{~mm}$ falling in this time. It begins to rain again around $8.00 \mathrm{pm}$, with $0.2 \mathrm{~mm}$ an hour falling for two hours.

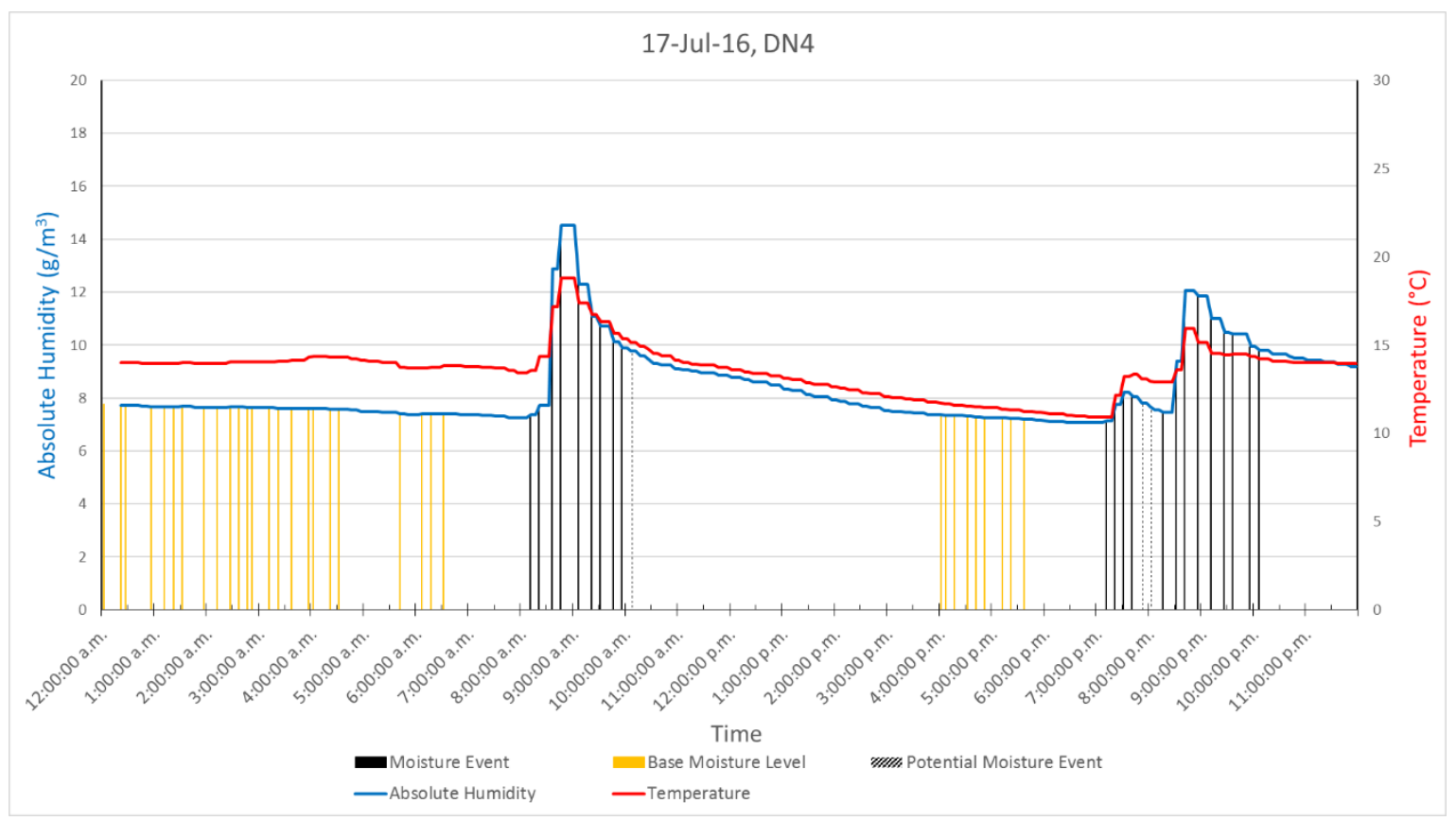

Figure 36 DN4, 17/07/16 - Sunday

The indoor temperature from $12.00 \mathrm{am}$ to $5.00 \mathrm{am}$ remained between $13.9^{\circ} \mathrm{C}$ and $14.3^{\circ} \mathrm{C}$, registering as a base moisture level for most of this time (Figure 36). The indoor absolute humidity was at $7.8 \mathrm{~g} / \mathrm{m}^{3}$ at $12.00 \mathrm{am}$ and $7.5 \mathrm{~g} / \mathrm{m}^{3}$ at $5.00 \mathrm{am}$. This low rate of change was not reflected in the outdoor conditions at the time. The rain from 3.00am to 4.00am resulted in a spike in absolute humidity that had no immediate, measurable impact on the indoor environment.

It is worth noting the indoor environment may react differently to changes in the outdoor climate depending on the time of day and the occupants' behaviour. The occupants may close all the windows in the house overnight, reducing the number of air changes per hour. More windows may be left open during the day, increasing the number of air changes per hour and increasing the impact of the outside climate. The number of air changes per hour will affect the quantity of moisture inside (McNeil \& Rupp, 2017). 
It rained again the next day, the $18^{\text {th }}$ of July, at $6.00 \mathrm{pm}$ and again at $10.00 \mathrm{pm}$. At $6.00 \mathrm{pm}$ the outdoor temperature drops from $9^{\circ} \mathrm{C}$ to $8^{\circ} \mathrm{C}$, while the relative humidity increases from $70 \%$ to $80 \%$. At $7.00 \mathrm{pm}$ the temperature and relative humidity return to previous levels, at $9^{\circ} \mathrm{C}$ and $70 \%$ humidity. A total of $0.6 \mathrm{~mm}$ of rain falls from $6.00 \mathrm{pm}$ until $7.00 \mathrm{pm}$, decreasing to $0.2 \mathrm{~mm}$ from $7.00 \mathrm{pm}$ to $8.00 \mathrm{pm}$. These return to their original levels as the rain stops, before dropping as the rain begins again at $9.00 \mathrm{pm}$. Only $0.2 \mathrm{~mm}$ per hour of rain falls at $9.00 \mathrm{pm}$. The temperature shows little change, although the relative humidity shows a slight increase from $70 \%$ to $74 \%$. These changes become more substantial when rainfall increases to $1.2 \mathrm{~mm}$ per hour at $10.00 \mathrm{pm}$. The temperature decreases from $9^{\circ} \mathrm{C}$ to $8^{\circ} \mathrm{C}$, while the relative humidity increases from $74 \%$ to $87 \%$.

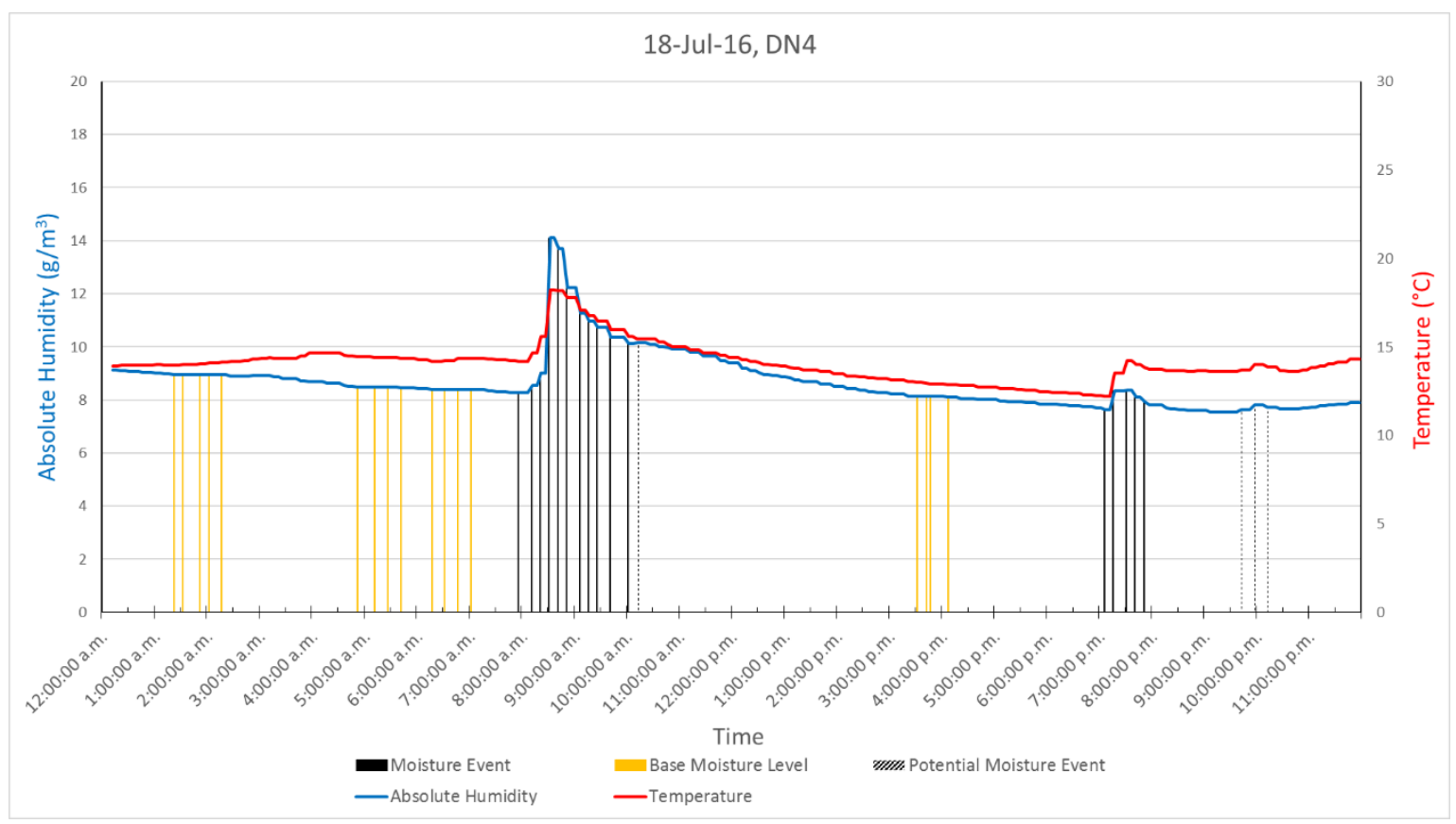

Figure 37 DN4, 18/07/16 - Monday

Once again, these changes in outside temperature and humidity have a very limited impact on the house. The absolute humidity does spike around 7.00pm and 10.00pm (Figure 37) when it is raining outside. However, the temperature also spikes at these times inside while it decreases outside. The inside absolute humidity during this period starts around $7.6 \mathrm{~g} / \mathrm{m}^{3}$, peaks at $8.3 \mathrm{~g} / \mathrm{m}^{3}$, and drops to $7.5 \mathrm{~g} / \mathrm{m}^{3}$. Considering the outside temperature decreased when it rained, it is unlikely the outdoor climate would affect the indoor humidity. Instead it would have either no impact or an entirely reversed impact on the indoor temperature. This makes it more likely the changes in absolute humidity in the house were self-contained 
moisture events that experienced little influence from what was happening outside at the time.

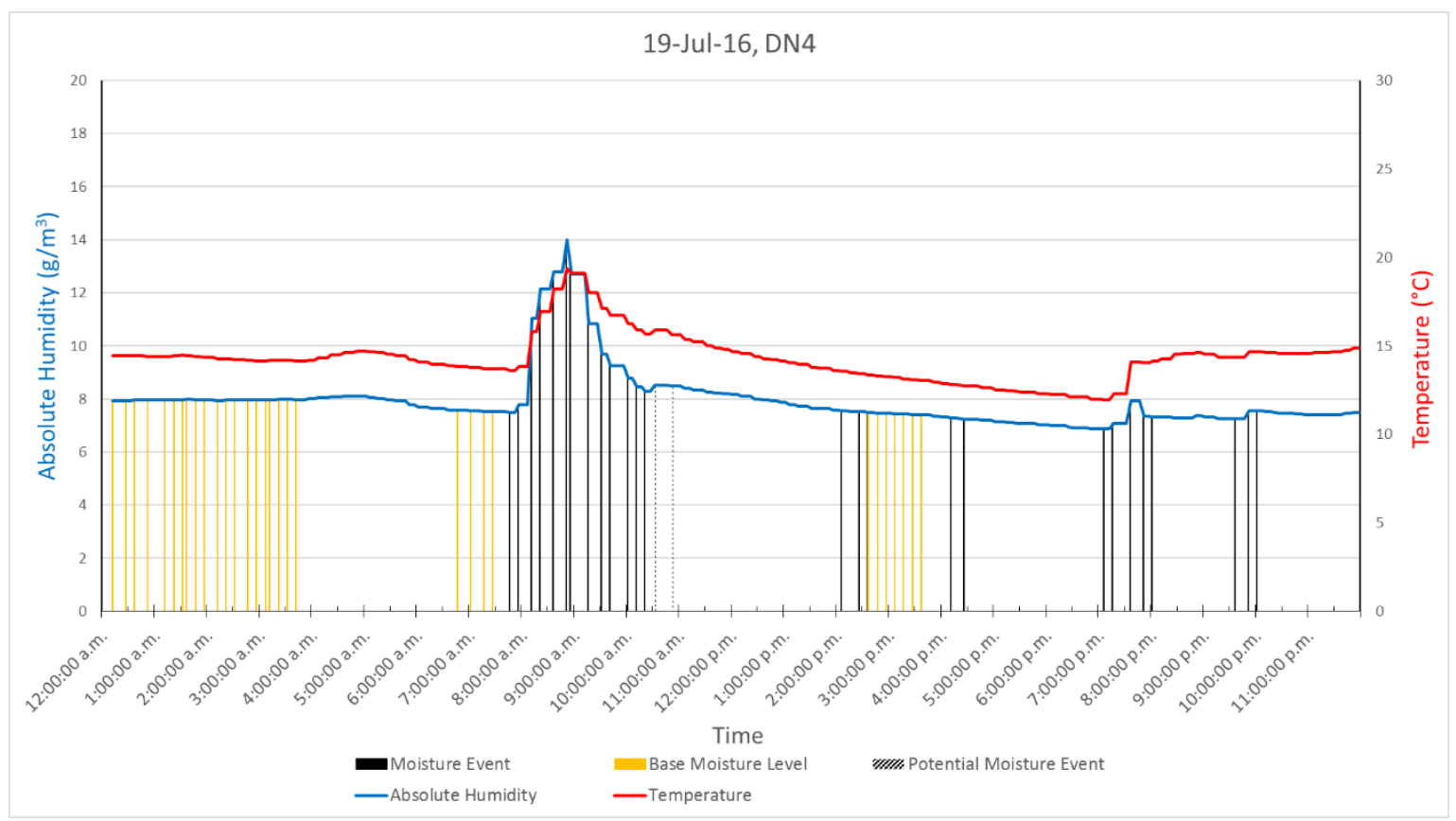

Figure 38 DN4, 19/07/16 - Tuesday

This is made even more likely when comparing these events with subsequent days. Tuesday the $19^{\text {th }}$, the next day, shows similar spikes at the same time $-7.00 \mathrm{pm}$ and just before $10.00 \mathrm{pm}$ (Figure 38). It did not rain on this day. These spikes in absolute humidity occur regardless of whether or not it was raining outside. This may indicate spikes in outdoor humidity are unlikely to cause spikes in absolute humidity inside this home.

While the overall trends with spikes and timing are the same, the original absolute humidity levels are lower than the measurements taken on the $18^{\text {th }}$. While the same period for the day before started at $7.6 \mathrm{~g} / \mathrm{m}^{3}$, peaked at $8.3 \mathrm{~g} / \mathrm{m}^{3}$, and dropped to $7.5 \mathrm{~g} / \mathrm{m}^{3}$, the $19^{\text {th }}$ of July starts at $6.8 \mathrm{~g} / \mathrm{m}^{3}$, peaks at $7.9 \mathrm{~g} / \mathrm{m}^{3}$, and drops to $7.2 \mathrm{~g} / \mathrm{m}^{3}$. This may indicate that while the outdoor climate has little impact on the peak and rate of decay of absolute humidity during moisture events, it may have a small, constant effect on the temperature and absolute humidity throughout the day. For example, on the $18^{\text {th }}$, despite sharing similar moisture events and rates of change as the $19^{\text {th }}$, the indoor environment consistently had $1 \mathrm{~g} / \mathrm{m}^{3}$ more moisture in the air at any given time. The exception to this pattern is the absolute humidity measured at the peak of the moisture events: 
- On the $18^{\text {th }}$ the peak for the morning moisture event occurred at 8.40am and reached $14.10 \mathrm{~g} / \mathrm{m}^{3}$. On the $19^{\text {th }}$ the peak for the same type of moisture event occurred at $8.50 \mathrm{am}$ and reached $14.00 \mathrm{~g} / \mathrm{m}^{3}$. This is a difference of $0.10 \mathrm{~g} / \mathrm{m}^{3}$.

- On the $18^{\text {th }}$ the peak for the evening moisture event occurred at 7.30am and reached $8.38 \mathrm{~g} / \mathrm{m}^{3}$. On the $19^{\text {th }}$ the peak for the same type of moisture event occurred at $7.35 \mathrm{pm}$ and reached $7.92 \mathrm{~g} / \mathrm{m}^{3}$. This is a difference of $0.46 \mathrm{~g} / \mathrm{m}^{3}$.

- On the $18^{\text {th }}$ the peak for the night moisture event occurred at $10.10 \mathrm{pm}$ and reached $7.73 \mathrm{~g} / \mathrm{m}^{3}$. On the $19^{\text {th }}$ the peak for the same type of moisture event occurred at $9.50 \mathrm{pm}$ and reached $7.56 \mathrm{~g} / \mathrm{m}^{3}$. This is a difference of $0.17 \mathrm{~g} / \mathrm{m}^{3}$.

This shows that, although measurements at the peak of moisture events from the $19^{\text {th }}$ were still consistently lower than the same measurements taken on the $18^{\text {th }}$, the difference in absolute humidity was smaller at this point than at any other point during the day. This suggests that while the outdoor climate is unlikely to cause sudden changes in the indoor climate, it will continue to influence the indoor conditions throughout the day. This affects almost everything except for indoor moisture events, which appear to introduce too much moisture for the outdoor climate to substantially influence.

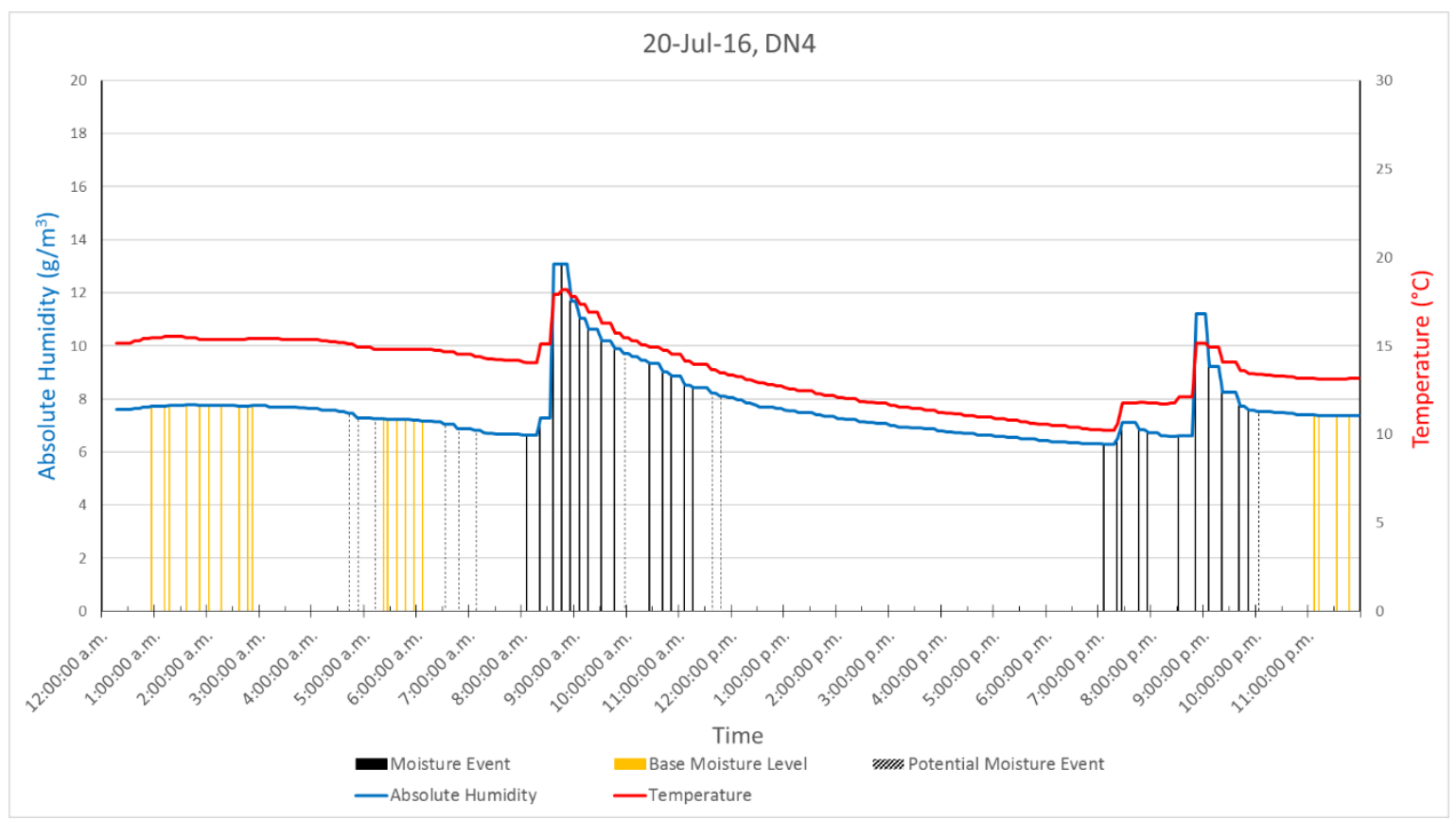

Figure 39 DN4, 20/07/16 - Wednesday

These results can be further tested by comparing them to measurements from the $20^{\text {th }}$ of July (Figure 39). On this day, $1.6 \mathrm{~mm}$ of rain per hour fell from $5.00 \mathrm{am}$ and continued until $7.00 \mathrm{am}$. There were a few more small showers of rain during the day at $11.00 \mathrm{am}$ and 
$1.00 \mathrm{pm}$. However, this was only at a rate of $0.2 \mathrm{~mm}$ per hour. There was no rainfall at night. The outdoor relative humidity peaked at $77 \%$ during the first shower and reached $75 \%$ around midday. At $7.00 \mathrm{pm}$ it had fallen to $66 \%$, and by $10.00 \mathrm{pm}$ it reached $52 \%$. This is reflected in the outside absolute humidity, which spiked to $5.97 \mathrm{~g} / \mathrm{m}^{3}$ at $5.00 \mathrm{am}$ when it started to rain. This fell to $4.73 \mathrm{~g} / \mathrm{m} 3$ by $7.00 \mathrm{am}$, when the rain had stopped. After $7.00 \mathrm{pm}$ the absolute humidity started to fall from $5.12 \mathrm{~g} / \mathrm{m}^{3}$, reaching $4.30 \mathrm{~g} / \mathrm{m}^{3}$ by $10.00 \mathrm{pm}$.

As was the case with the previous days, these spikes in humidity did not line up with the spikes in humidity indoors, which happened at $8.30 \mathrm{am}, 7.30 \mathrm{pm}$, and $9.00 \mathrm{pm}$. This trend demonstrates the outdoor environment likely has little impact on moisture events happening in the bathroom of house DN4, with the absolute humidity at the peak (Table 7) and end (Table 8) of moisture events remaining more consistent than the absolute humidity at the start (Table 6). However, it does affect the base temperature and moisture levels of the house throughout the day. This is important, as it allows moisture events to be analysed independently of minor changes in the weather, allowing more direct comparisons to be made between different events. To further study this, all available data would need to be compared with weather data to analyse what impact the weather had on moisture events. This is beyond the scope of this study.

\begin{tabular}{|l|c|c|}
\hline \multicolumn{1}{|c|}{ Event Start } & Time & Absolute Humidity \\
\hline DN4, 01/07/16 & $8.05 \mathrm{am}$ & $6.7 \mathrm{~g} / \mathrm{m}^{3}$ \\
\hline DN4, 02/07/16 & $8.10 \mathrm{am}$ & $7.2 \mathrm{~g} / \mathrm{m}^{3}$ \\
\hline DN4, 03/07/16 & $8.10 \mathrm{am}$ & $11.7 \mathrm{~g} / \mathrm{m}^{3}$ \\
\hline DN4, 04/07/16 & $8.10 \mathrm{am}$ & $8.4 \mathrm{~g} / \mathrm{m}^{3}$ \\
\hline DN4, 05/07/16 & $8.05 \mathrm{am}$ & $8.6 \mathrm{~g} / \mathrm{m}^{3}$ \\
\hline DN4, 06/07/16 & $8.15 \mathrm{am}$ & $7.1 \mathrm{~g} / \mathrm{m}^{3}$ \\
\hline DN4, 07/07/16 & $8.05 \mathrm{am}$ & $6.9 \mathrm{~g} / \mathrm{m}^{3}$ \\
\hline DN4, 08/07/16 & $8.05 \mathrm{am}$ & $7.2 \mathrm{~g} / \mathrm{m}^{3}$ \\
\hline
\end{tabular}

Table 6 Sample of Measurements from Start of Moisture Events

\begin{tabular}{|l|c|c|}
\hline \multicolumn{1}{|c|}{ Event Peak } & Time & Absolute Humidity \\
\hline DN4, 01/07/16 & $8.50 \mathrm{am}$ & $14.4 \mathrm{~g} / \mathrm{m}^{3}$ \\
\hline DN4, 02/07/16 & $8.45 \mathrm{am}$ & $15.0 \mathrm{~g} / \mathrm{m}^{3}$ \\
\hline DN4, 03/07/16 & $8.25 \mathrm{am}$ & $16.9 \mathrm{~g} / \mathrm{m}^{3}$ \\
\hline DN4, 04/07/16 & $8.35 \mathrm{am}$ & $16.4 \mathrm{~g} / \mathrm{m}^{3}$ \\
\hline DN4, 05/07/16 & $8.35 \mathrm{am}$ & $14.0 \mathrm{~g} / \mathrm{m}^{3}$ \\
\hline DN4, 06/07/16 & $8.45 \mathrm{am}$ & $13.5 \mathrm{~g} / \mathrm{m}^{3}$ \\
\hline DN4, 07/07/16 & $8.40 \mathrm{am}$ & $13.1 \mathrm{~g} / \mathrm{m}^{3}$ \\
\hline DN4, 08/07/16 & $8.50 \mathrm{am}$ & $12.4 \mathrm{~g} / \mathrm{m}^{3}$ \\
\hline
\end{tabular}

Table 7 Sample of Measurements from Peak of Moisture Events 


\begin{tabular}{|l|c|c|}
\hline \multicolumn{1}{|c|}{ Event end } & Time & Absolute Humidity \\
\hline DN4, 01/07/16 & $9.35 \mathrm{am}$ & $10.2 \mathrm{~g} / \mathrm{m}^{3}$ \\
\hline DN4, 02/07/16 & $10.05 \mathrm{am}$ & $10.6 \mathrm{~g} / \mathrm{m}^{3}$ \\
\hline DN4, 03/07/16 & $9.40 \mathrm{am}$ & $12.5 \mathrm{~g} / \mathrm{m}^{3}$ \\
\hline DN4, 04/07/16 & $10.00 \mathrm{am}$ & $11.2 \mathrm{~g} / \mathrm{m}^{3}$ \\
\hline DN4, 05/07/16 & $10.15 \mathrm{am}$ & $8.1 \mathrm{~g} / \mathrm{m}^{3}$ \\
\hline DN4, 06/07/16 & $9.45 \mathrm{am}$ & $10.1 \mathrm{~g} / \mathrm{m}^{3}$ \\
\hline DN4, 07/07/16 & $10.00 \mathrm{am}$ & $10.4 \mathrm{~g} / \mathrm{m}^{3}$ \\
\hline DN4, 08/07/16 & $9.35 \mathrm{am}$ & $9.5 \mathrm{~g} / \mathrm{m}^{3}$ \\
\hline
\end{tabular}

Table 8 Sample of Measurements from End of Moisture Events

\subsubsection{Discussion}

Evaluating how much the humidity changed during these events, as well as how long this change took place over, provides a good starting point for the analysis. As demonstrated in the weather comparison analysis, the outdoor conditions will affect the temperature and humidity at the start and finish of the event, but the event itself will remain reasonably consistent. Because of these differences in outdoor conditions, some days may have identical rates of change but take longer to reach their peaks as they had a lower starting point. This makes the rate of change, rather than the length the change took place over, more important for comparing events.

It is also important to consider overnight conditions. For example, on $03 / 07 / 16$, there was an event starting at 3.30am which peaked at 4.40am and ended at 5.20am. As the regular moisture event began only three hours later, the room conditions did not have enough time to return to normal. This meant the regular event was heavily influenced by the additional humidity and higher temperature, resulting in a significantly higher peak and more dramatic rate of change.

\subsubsection{Rates of Change}

This section provides a more detailed analysis of each event, showing each of their rates of change. The 'rate of change' (RoC) is concerned with three different points:

- while moisture increases at the start of the event (RoC A);

- while moisture decreases at the end of the event (RoC B); and

- the period following the event before the absolute humidity in the room returns to base (RoC C).

The rate of change is calculated: 
- for the start of the event by taking the average increase in absolute humidity between the event start and the event peak;

- for the end of the event by taking the average decrease in absolute humidity between the event peak and the event end; and

- following the event by taking the average decrease in absolute humidity between the end of the event and either the base moisture level or the next moisture event, depending on what happens first.

RoC B and RoC C are considered separately as these two periods behave very differently, despite both involving the absolute humidity decreasing. After the moisture event reaches its peak and the moisture source is no longer producing moisture, this level of absolute humidity cannot be maintained. Regardless of whether moisture controls are active, the absolute humidity decreases as the room reaches an equilibrium with adjacent spaces and some of the moisture in the air falls or condenses.

Additionally, this is the period where it is most likely active control systems, such as extract fans, were used. These rapidly remove moisture from the indoor environment, decreasing absolute humidity at a far higher rate than would normally be achieved by passive systems. This means the rate of change is affected by entirely different factors, making calculating an average rate of change for the entire period of decreasing absolute humidity largely meaningless.

The length of time the peak of the moisture event lasts is also an important factor to record. This is the section of the moisture event on either side of the highest recorded measurement where the absolute humidity is no longer increasing or decreasing by the specified moisture event rate of change (3\%). If there are no measurements on either side meeting these criteria, then the peak length was measured from the recording taken directly before the peak to the recording taken directly following the peak.

\begin{tabular}{|l|c|c|}
\hline \multicolumn{1}{|c|}{ DN4, 01/07/16 } & Time & Absolute Humidity \\
\hline Event Start & $8.05 \mathrm{am}$ & $6.7 \mathrm{~g} / \mathrm{m}^{3}$ \\
\hline Event Peak & $8.50 \mathrm{am}$ & $14.4 \mathrm{~g} / \mathrm{m}^{3}$ \\
\hline Event End & $9.35 \mathrm{am}$ & $10.2 \mathrm{~g} / \mathrm{m}^{3}$ \\
\hline Base Moisture Level & $8.05 \mathrm{pm}$ & $6.4 \mathrm{~g} / \mathrm{m}^{3}$ \\
\hline
\end{tabular}

Table 9 Measurements of a Single Moisture Event 


\begin{tabular}{|l|c|c|c|}
\hline \multicolumn{1}{|c|}{ DN4, 01/07/16 } & Start Time & End Time & Duration \\
\hline Event Start & $8.05 \mathrm{am}$ & $8.50 \mathrm{am}$ & 45 \\
\hline Event Peak & $8.50 \mathrm{am}$ & $9.05 \mathrm{am}$ & 15 \\
\hline Event End & $9.05 \mathrm{am}$ & $9.35 \mathrm{am}$ & 30 \\
\hline Base Moisture Level & $9.35 \mathrm{am}$ & $8.05 \mathrm{pm}$ & 630 \\
\hline
\end{tabular}

Table 10 Duration of Each Stage of a Single Moisture Event

RoC A consists of a change of $7.7 \mathrm{~g} / \mathrm{m}^{3}$ over 45 minutes (Table 9) (Table 10). This gives the period a rate of change of $0.17 \mathrm{~g} / \mathrm{m}^{3}$ per minute.

RoC $B$ consists of a change of $4.2 \mathrm{~g} / \mathrm{m}^{3}$ over 45 minutes (Table 9) (Table 10). This gives the period a rate of change of $0.09 \mathrm{~g} / \mathrm{m}^{3}$ per minute.

RoC C consists of a change of $3.8 \mathrm{~g} / \mathrm{m}^{3}$ over 630 minutes (Table 9) (Table 10). This gives the period a rate of change of $0.01 \mathrm{~g} / \mathrm{m}^{3}$ per minute.

\subsection{Results for Additional Consideration}

This section provides a summary of some of the most useful information gathered from this initial data test. It provides insight into areas that were not able to be fully explored in the methodology or could only be briefly considered in the analysis. This was either due to a lack of available data or the area being too complex to be fully developed within the scope of this research. These should be considered for future research.

\subsubsection{Indoor Temperature vs Outdoor Temperature}

As identified when reviewing the impact of outdoor weather conditions on indoor moisture events, 6.6.2 Comparison to Weather, the external weather influences the 'base' indoor temperature and humidity. This relationship needed to be better understood to accurately analyse the full impact this would have on moisture events. Any relationship between the two would need to take differences in seasons and microclimates into account. While these trends may hold true for the month of data analysed, it may not to the case under different conditions, such as during summer, but this was beyond the scope of this research.

\subsubsection{Moisture Event End and Base Moisture Level}

There is an important distinction between the end of a moisture event and the indoor environment returning to 'base moisture level'. The 'end of the moisture event' refers to the point where a moisture source is no longer contributing large amounts of moisture, or where ventilation systems are no longer removing moisture at a high rate. This would mean 
the moisture event will be defined as ending when the absolute humidity's rate of change returns to within a certain range. This is defined as 3\%, keeping the rate of change required to qualify measurements as being part of a moisture event consistent for the entire duration of the event.

The base moisture level on the other hand is when that moisture event is no longer having any impact on the indoor environment. This means while the absolute humidity is decreasing at a normal rate (not assisted by mechanical ventilation or temporarily increased natural ventilation from sources such as opened windows), it will no longer qualify as a moisture event. However, the indoor environment will not qualify as having reached base moisture level' until the absolute humidity returns to the same level it would have been if no moisture event had taken place. As discussed in 6.3.3.3 Falsely Identified Base Moisture Levels, depending on the indoor environment and how well heated and ventilated it is, the absolute humidity may not have enough time to decrease to base moisture levels before the next moisture event starts. This means moisture events will not necessarily be able to be consistently compared with subsequent base moisture levels.

\subsubsection{Differences in Moisture Events}

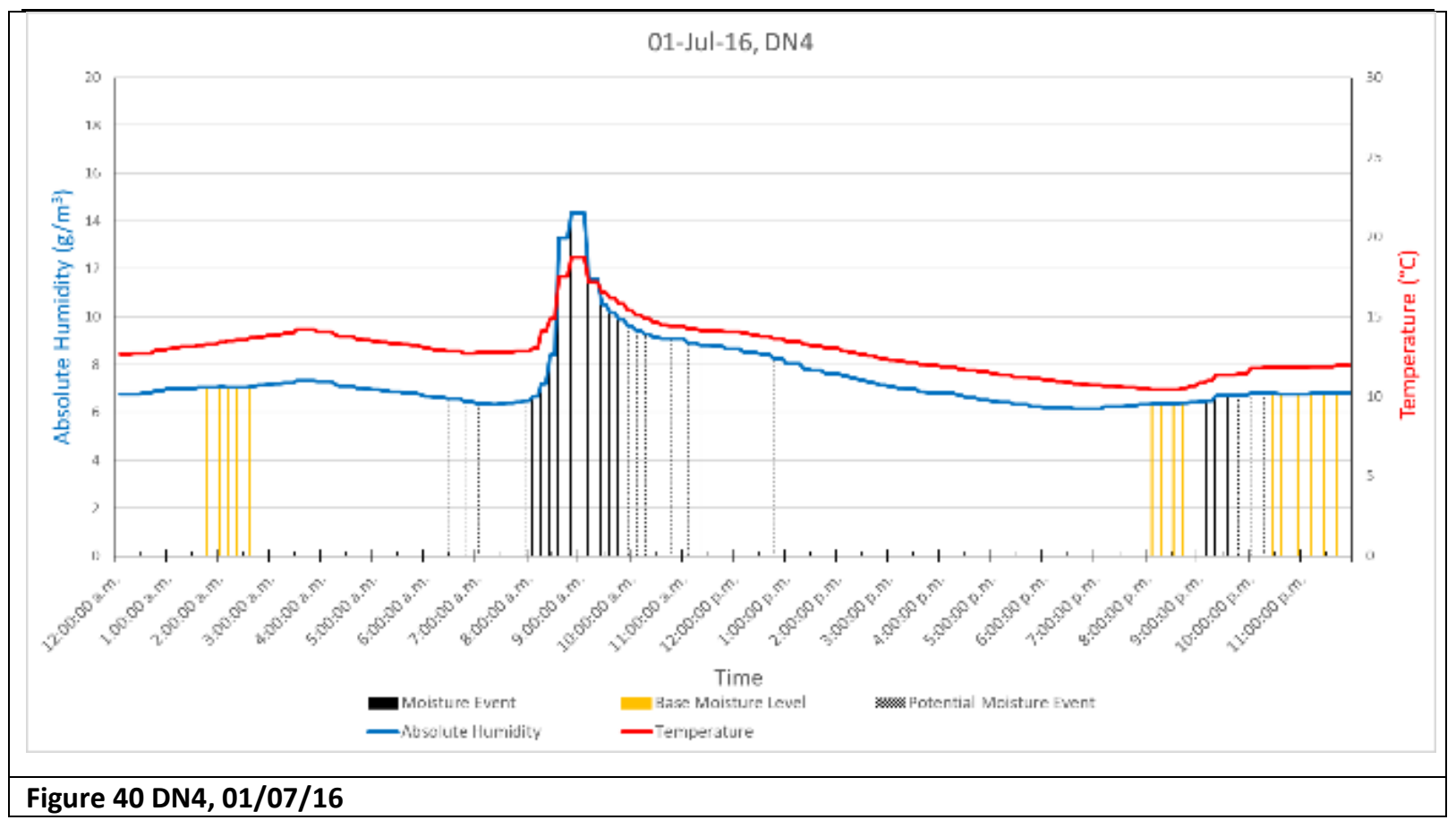




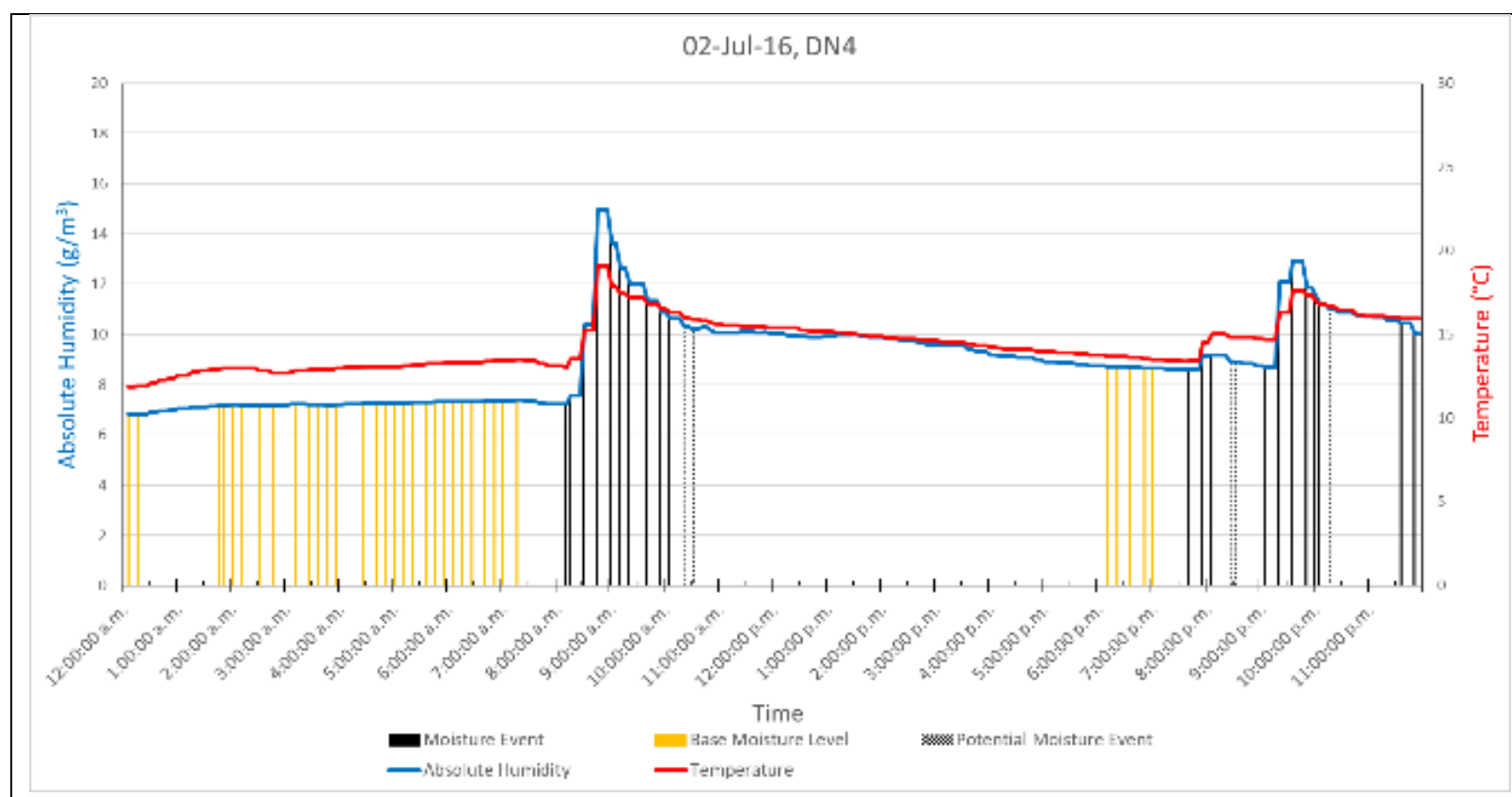

Figure 41 DN4, 02/07/16

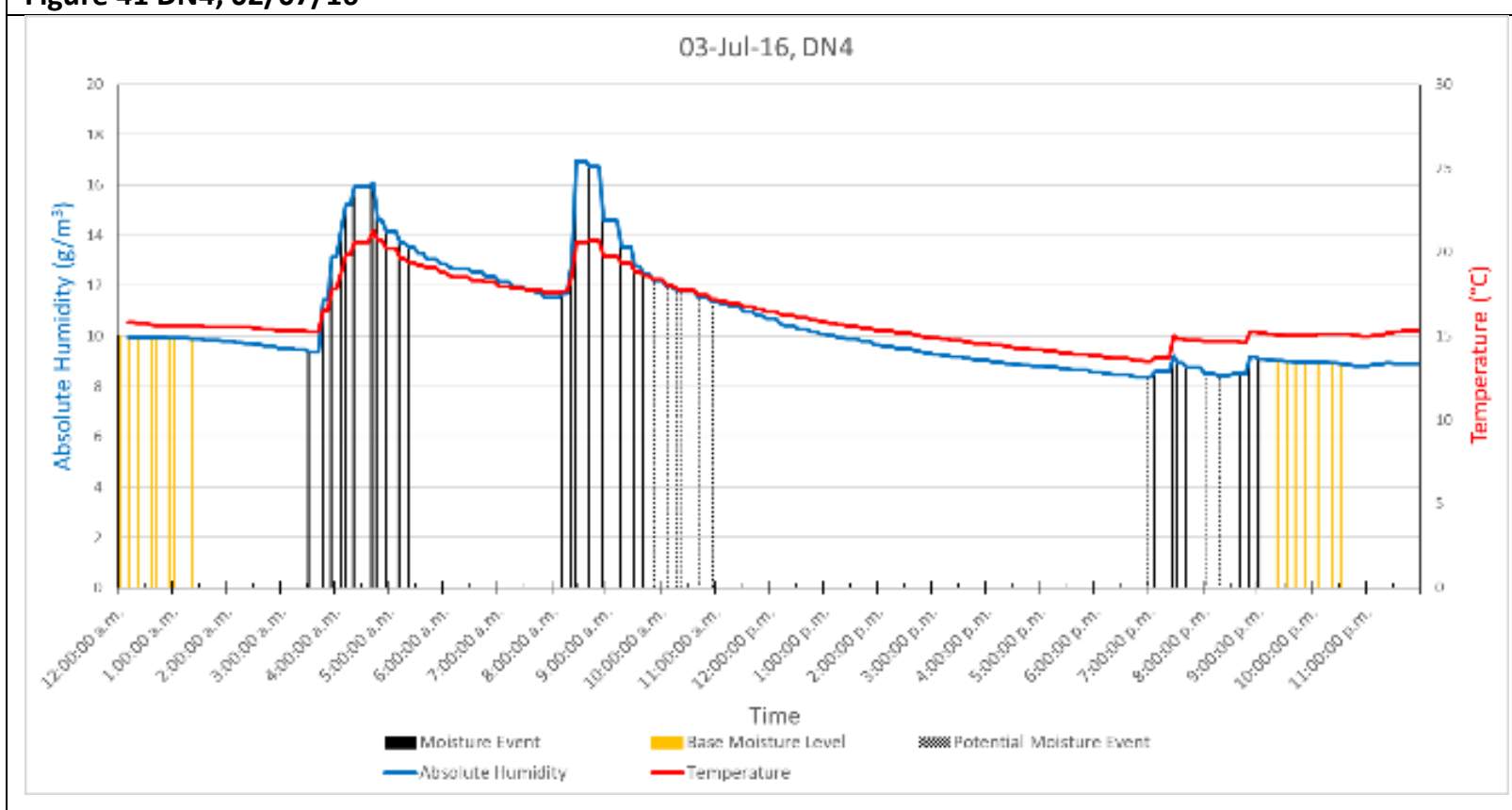

Figure 42 DN4, 03/07/16 


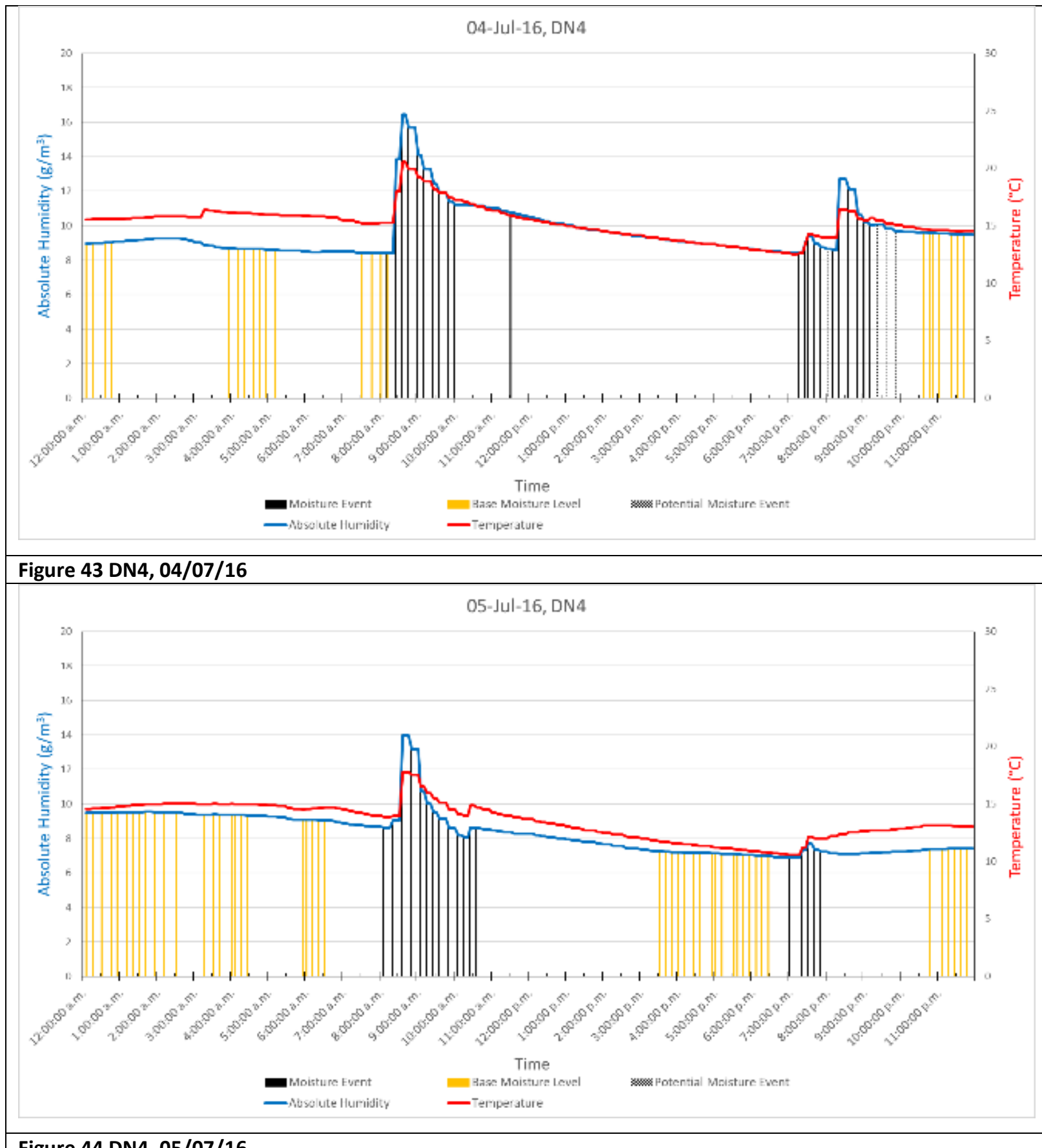

Figure 44 DN4, 05/07/16 


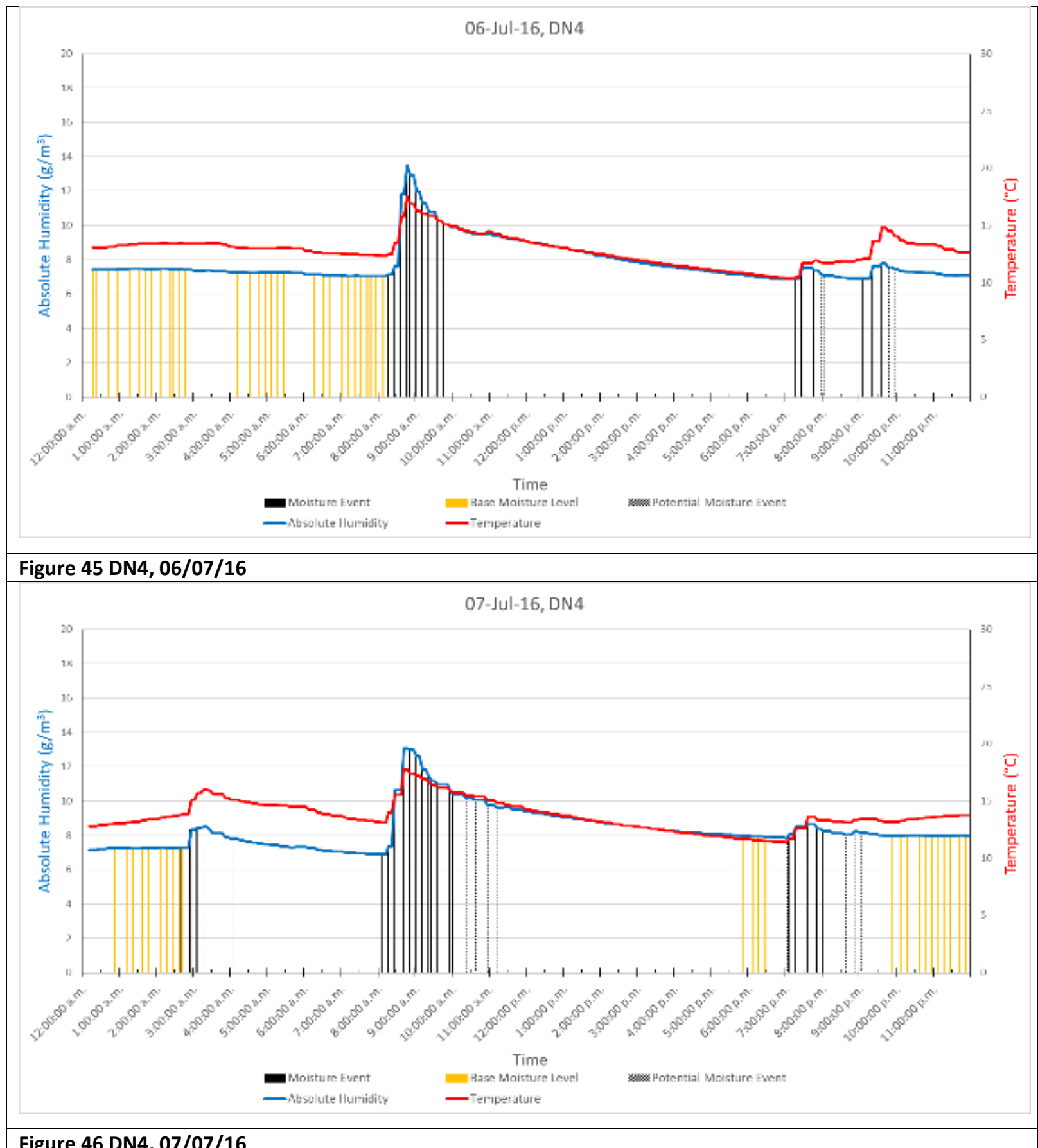

Figure 46 DN4, 07/07/16 


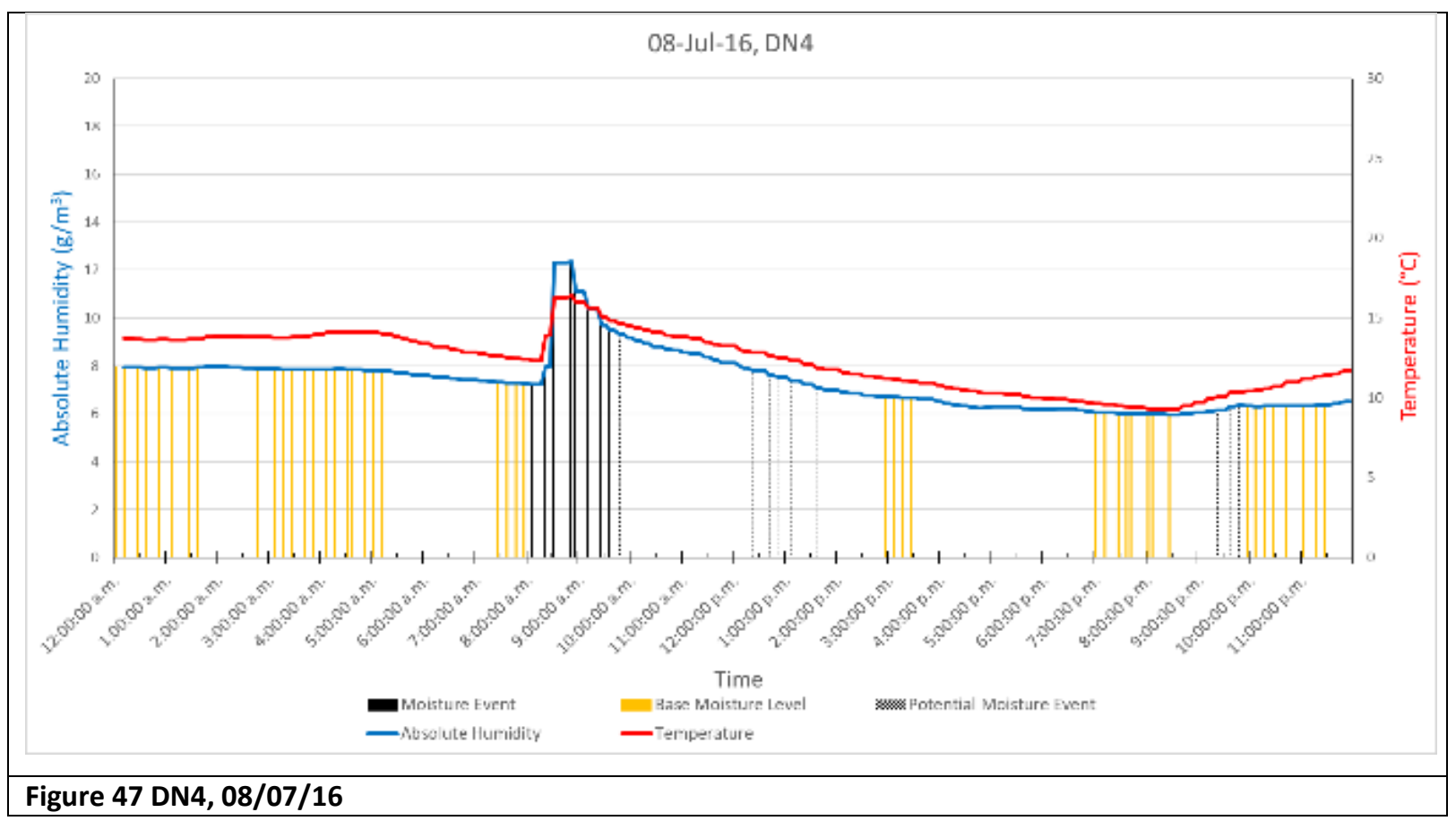

The data gathered on moisture events from a single house need to be as similar as possible to be compared. Ideally, this means moisture events would happen at the same time, under the same environmental conditions, and with the same occupants present. The available data does not include information this detailed, and even if it did, moisture events this regular may not accurately reflect typical New Zealand homes.

Assumptions were made based on the data available. There is no information on which occupants were present at the time. While basic environmental information was available and could be compared to the outdoor conditions at the time, there is not enough detail provided to accurately predict whether two events occurred under reasonably similar circumstances. Time is the most useful information available, requiring very few assumptions before being analysed.

Moisture events that consistently happen at a similar time each day imply this event is part of the occupants' daily schedule. This makes it safe to assume most variables are reasonably similar between days. In DN4, the 8.00am moisture event is very consistent. This can be seen in the sample of one week of data provided (Figure 40 - Figure 47). Figures 40 to 47 are on the same vertical scales. The absolute humidity (left scale) ranges from 0 to $20 \mathrm{~g} / \mathrm{m}^{3}$, while the Temperature (right scale) is from $0^{\circ} \mathrm{C}$ to $30^{\circ} \mathrm{C}$. 
Almost every day, a moisture event will begin at $8.00 \mathrm{am}$, peak just before $9.00 \mathrm{am}$, and finish around 10.00am. This consistency provides the ideal moisture event to analyse. A minimum of seven days would need to be analysed to demonstrate how this event fits into the occupants' weekly schedule. Further studies could focus on isolating these regular events, carrying out a full analysis on how these vary and impact the indoor environment.

\subsubsection{Additional Data for Comparisons}

There are several factors not provided in the available data that would help expand on the analysis of each house and provide information on how different types of bathrooms tend to perform. This includes factors such as differences in extractor fans, windows, type of occupants, year of construction, and whether any additional forms of ventilation or moisture control are present.

Even without information on these factors, assumptions on performance can be made by relating results back to the literature. This includes considering how moisture sources behave and the quantity of moisture they are expected to produce. By comparing these to other sources of data such as the HEEP study, it can be shown whether they are acting as anticipated, whether they are at safe levels, or if they could potentially be modelled in future studies. More importantly, this can show how these spaces are likely to continue to perform and whether they are likely to put occupants at risk.

\subsection{Data Test Review}

\subsubsection{Exploratory Data Analysis}

Understanding the data readability is a key consideration. Providing a clear representation of the indoor environment helps to better review and understand changes in temperature and absolute humidity in a wider context. It also provides a means to double-check information, presenting errors and unusual readings in a way that makes them stand out from other measurements.

The line graph which has been developed uses two vertical axes to allow temperature and absolute humidity to be easily read, understood, and compared with each other. The absolute humidity, as the focus of the analysis, is listed on the primary axis and ranges 85 
between $0 \mathrm{~g} / \mathrm{m}^{3}$ and $20 \mathrm{~g} / \mathrm{m}^{3}$. The temperature is listed on the secondary axis and ranges between $0^{\circ} \mathrm{C}$ and $30^{\circ} \mathrm{C}$. This may be an issue for houses that exceed these levels.

This requires a balance between consistency between houses and visual clarity of data. Increasing the range would result in data being presented in a smaller area for most houses, making it more difficult to read and any changes throughout the day less obvious. The data would be easier to read if every graph's axis was sized appropriately depending on what measurements were presented. However, this would make data more difficult to compare. Providing a consistent scale allows the reader to immediately understand the information the graph is providing in a wider context, as it can be directly compared to the previous graphs shown.

The data must be presented in the way that best answers the question being asked. In this situation, the most important detail is how the absolute humidity changes throughout the day. The inclusion of a bar graph highlighting moisture events addresses this. This provides three key pieces of information:

- It immediately identifies the measurement it is associated with. It lines up with the absolute humidity, clearly communicating the relationship between the two.

- It highlights periods with increased rates of change without making the absolute humidity line any less readable with cluttered notes or potentially subtle or confusing changes in colour.

- It shows how frequently the measurements were taken compared to the graph itself. The closer the bars are together, the more frequently measurements were taken during the event. This provides more context for analysing the results.

The graphs must also communicate how they relate to any other analysis carried out on the measurements. The main example of this in the pilot study was the analysis of whether the weather impacts each moisture event. To demonstrate how the weather and indoor environment may be related, the weather was presented using the same type of graph as the bathroom. This made any differences or similarities easier to read, providing context for assumptions made on whether to account for the impact of weather in the analysis. For example, when the outdoor temperature increases (while the indoor temperature either 
remains constant or changes alongside it) presenting this information on the same type of graph helps to communicate the relationship between the two more clearly.

\subsubsection{Process Order}

The initial data analysis began by analysing the impact of weather. This was done to account for factors influencing the temperature and absolute humidity before analysing them. This may not be the most effective approach. The purpose of the study is to review how houses perform in reality, demonstrating whether moisture being effectively controlled. Immediately taking the outdoor conditions into account and analysing the indoor conditions independently of external conditions may not be the best approach. Considering that fully understanding the impact of weather on moisture events is beyond the scope of this research, introducing this at the start of the analysis would result in conclusions and assumptions being based on data that had not been fully explored.

An alternative solution is to analyse the indoor environment first, noting whether the conditions are healthy. Once this has been done the external conditions would be compared to a day where either few or no moisture events occurred, indicating the impact the external conditions have on the indoor environment. By analysing the data beforehand, the results would reflect what the occupants were actually experiencing, providing a better indication of whether or not the indoor conditions were being maintained at healthy levels. Analysing the impact of external conditions after this would simply be a means of differentiating the impact of occupants from the weather and house condition. However, comparing the external conditions to the indoor environment does have downsides. Since the houses will only be analysed for a limited period, analysing the results without taking external conditions into account means findings may only apply to the season the measurements were recorded. This means the period would need to be during the worst possible conditions (likely during winter months) so the results would reflect how healthy the indoor environment was during the worst-case scenario. 


\subsubsection{Potential Issues}

\subsubsection{Using DN4 for the Initial Data Test}

While the data analysis provided useful information that contributed towards processing and analysing the remaining data, this approach assumed DN4 was representative of most houses measured for the study. There was the risk other houses may not have comparable results, making the data processing template ineffective. This was mitigated by testing other houses throughout this process, comparing results and ensuring moisture events behaved in similar ways.

\subsubsection{Five Minute Periods}

The method for standardising measurements taken at irregular intervals was described in 6.5.3 Processing Data. This involved sorting measurements into five minute periods. One potential downside of this is when measurements were only placed into certain five minute periods by a matter of seconds. This could misrepresent how far apart measurements were taken, showing measurements, whether taken 30 seconds apart or 9 minutes and 59 seconds apart as only being five minutes apart.

\subsubsection{Analysis}

As the measurements are taken over a long period, it would take more time than is available to analyse each day individually for all available houses completely. This means that identifying trends are heavily reliant on the accuracy of the average moisture events identified during the full-scale data analysis.

One section of the initial data analysis, 6.7.3 Differences in Moisture Events, compared moisture events taking place at similar times each day. Assumptions used for the moisture event analysis were based on the event's consistency. It ran at similar times each day, implying it was part of the occupants' daily schedule. This made it more likely the variables surrounding the moisture events were similar each day, making the events easier to compare. This same assumption may not necessarily apply to moisture events in other houses. If moisture events were to happen at different times, different factors influencing the results would become more difficult to take into account. This would make comparing daily events difficult. 
However, these events could be used for a different type of analysis. Instead of comparing small differences in nearly identical moisture events, larger differences could be analysed based on the different times the moisture event took place. If one house had an event that could be identified as a bath or shower taking place each day at any one of five different times, the events could be grouped according to start and finish times. These could then be used to generate an 'average event'. Comparing average events would not provide detailed information on minute-to-minute differences, but it may provide useful information on how events are dealt with depending on the time of day.

For example, moisture events which occur before occupants leave the house may take longer to decay than a similar moisture event occurring when the occupants return, as the windows may not be left open when nobody is at home. This would rely on being able to identify events occurring at different times as being caused by the same source. If this cannot be safely assumed, then comparing events from throughout the day will be far less insightful. Events that cannot be identified may be compared by using their starting points, peaks, and end points as a measure of consistency. This would require a lot of assumptions and any results produced would provide little indication of what was taking place inside.

\subsubsection{Weather}

The weather is based on NIWA data which provides a measurement for the region as a whole. This does not take into account changes based on the specific area of the house created by the microclimate. The measurements are taken hourly. This makes it difficult to compare to the indoor measurements which are taken at far smaller intervals. The NIWA data provides the temperature and relative humidity, but not the absolute humidity. The information provided by BRANZ does not include the exact location of each house for privacy reasons, meaning assumptions would need to be made when selecting which weather station should be used for each area.

\subsubsection{Differences in Occupancy}

The house analysed for this initial data test, DN4, had consistent daily moisture events suggesting the house was occupied most days. For the full-scale data testing, this was less likely to be the case. The number of occupants could vary from day to day, potentially making it more difficult to analyse a typical day. This presents both advantages and disadvantages that must be considered to analyse each house accurately. 
Days where the house was unoccupied provide a baseline to compare with other results. These days could also be used to verify the impact of weather on the internal environment. If these periods of disuse are regular, then this would contribute to the analysis of occupant contribution to a healthy indoor environment in two different ways:

- A day without moisture events may act as a 'hard reset' for moisture in the room, giving it time to return to base moisture levels (assuming passive natural ventilation and heating is enough to deal with any excess moisture). This means the impact of subsequent moisture events can be analysed without requiring additional assumptions to be made on the impact of recent moisture events. If a single day is not enough time for the indoor environment to return to base moisture levels, then this also contributes towards the analysis of the house, showing passive natural ventilation alone is not having a meaningful impact on the environment.

- These periods will show what changes in temperature and humidity are actually being caused by occupants. If a spike in temperature occurs every morning, then it may be assumed to be caused by direct sunlight heating the indoor environment. However, if this change does not take place when the house does not appear to be occupied, then this may suggest the increase is caused by heaters being turning on and warming the house.

Issues may occur when there are large numbers of days with no apparent occupant use. This may result in a lack of useful data or would mean a typical day of occupant usage does not suggest what happens on most days. It may suggest the house is not unoccupied during these times and the occupants' daily routine instead happens to not result in a moisture event most of the time. These situations would be difficult to accurately identify and analyse, and a variety of assumptions would need to be made.

Alternatively, there may be some days where a greater number of people are present in the house. These days are difficult to identify accurately. Indicators may include additional or extended moisture events, but there is not enough data to identify the presence of additional occupants with absolute certainty. If these periods could be identified, then this would provide an excellent opportunity to analyse how well the indoor environment copes with the most extreme conditions it will typically face. This may highlight situations where 
Moisture in New Zealand Bathrooms

heating and ventilation is normally sufficient to maintain a healthy environment, but no longer meets demand once additional occupants create more moisture events to deal with each day. 
Alister Stubbe 


\section{Chapter 7: Full-Scale Data Processing}

\subsection{Selecting Data for Analysis}

\subsubsection{House Measurement Periods}

\begin{tabular}{|c|c|c|c|c|c|c|c|c|c|c|c|}
\hline Region & Simplified ID & Apr & May & Jun & Jul & Aug & Sep & Oct & Nov & Dec & Jan \\
\hline \multirow[t]{9}{*}{ Dunedin } & DN1 & & & & & & & & & & \\
\hline & DN2 & & & & & & & & & & \\
\hline & DN3 & & & & & & & & & & \\
\hline & DN4 & & & & & & & & & & \\
\hline & DN5 & & & & & & & & & & \\
\hline & DN6.1 & & & & & & & & & & \\
\hline & DN6.2 & & & & & & & & & & \\
\hline & DN7 & & & & & & & & & & \\
\hline & DN8 & & & & & & & & & & \\
\hline \multirow[t]{11}{*}{ Wellington } & WN1 & & & & & & & & & & \\
\hline & WN2 & & & & & & & & & & \\
\hline & WN3 & & & & & & & & & & \\
\hline & WN4 & & & & & & & & & & \\
\hline & WN5 & & & & & & & & & & \\
\hline & WN6 & & & & & & & & & & \\
\hline & WN7 & & & & & & & & & & \\
\hline & WN8.1 & & & & & & & & & & \\
\hline & WN8.2 & & & & & & & & & & \\
\hline & WN9 & & & & & & & & & & \\
\hline & WN10 & & & & & & & & & & \\
\hline \multirow[t]{4}{*}{ Blenheim } & BN1.1 & & & & & & & & & & \\
\hline & BN1.2 & & & & & & & & & & \\
\hline & BN2 & & & & & & & & & & \\
\hline & BN3 & & & & & & & & & & \\
\hline \multirow[t]{2}{*}{ Nelson } & NL1 & & & & & & & & & & \\
\hline & NL2 & & & & & & & & & & \\
\hline \multirow[t]{3}{*}{ Motueka } & MT1 & & & & & & & & & & \\
\hline & MT2.1 & & & & & & & & & & \\
\hline & MT2.2 & & & & & & & & & & \\
\hline \multirow[t]{2}{*}{ Lower Hutt } & LH1 & & & & & & & & & & \\
\hline & LH2 & & & & & & & & & & \\
\hline Porirua & PR1 & & & & & & & & & & \\
\hline \multicolumn{12}{|c|}{ Table 11 Measurement Periods } \\
\hline Colour/Column & Example & & & & & Meani & & & & & \\
\hline
\end{tabular}




\begin{tabular}{|l|l|l|}
\hline $\begin{array}{l}\text { Red } \\
\text { Simplified ID }\end{array}$ & DN5 & $\begin{array}{l}\text { There is an insufficient number of measurements during the selected } \\
\text { months for further analysis to take place. This house will not be } \\
\text { included in the comparisons and results. }\end{array}$ \\
\hline $\begin{array}{l}\text { Orange } \\
\text { Simplified ID }\end{array}$ & DN3 & $\begin{array}{l}\text { No moisture events took place during the selected months. This is } \\
\text { potentially due to the house being unoccupied during this time. This } \\
\text { house will not be included in the comparisons and results. }\end{array}$ \\
\hline $\begin{array}{l}\text { Green } \\
\text { Month }\end{array}$ & & $\begin{array}{l}\text { There are at least 3 weeks of consistent measurements for this } \\
\text { month. }\end{array}$ \\
\hline $\begin{array}{l}\text { Yellow } \\
\text { Month }\end{array}$ & $\begin{array}{l}\text { There are less than 3 weeks of consistent measurements for this } \\
\text { month. }\end{array}$ \\
\hline $\begin{array}{l}\text { Blank Cell } \\
\text { Month }\end{array}$ & No measurements were taken for this month. \\
\hline
\end{tabular}

Table 12 'Measurement Periods' Key

\subsubsection{Determining Houses for Further Analysis}

The initial data test used measurements taken from one house gathered over a period of one month. The full set of data contains data gathered from houses around the country (Table 11). The first step is to limit the analysis to houses with all necessary measurements and information available. BRANZ provided a bulk set of measurements for all measured houses as well as a key linking each ID number to a region and, in some cases, providing additional information about the bathroom. The houses were given a simplified ID code in the format [2-Letter Region Code] [House Number].

As shown in Table 11, measurements for a total of 32 bathrooms in 28 houses were provided. Measurements were taken over a period ranging from two months to six months. To allow results to be better compared with each other, the analysis was carried out on measurements taken within the same time frame. This helped mitigate various potential issues. Any differences in occupant activity based on the time of year are reduced.

\subsubsection{Selecting Analysis Period}

A sample of two months of data was used for each house. The August-September period included the greatest number of results (Table 11 Measurement Periods. Only one house, BN3, had no data measured over this period. One house, LH1, had several gaps during this period. One house, DN3, did not have moisture events recorded over this period. Two houses, DN5 and PR1, had only a few weeks of measurements taken over this period. Three houses, DN7, DN8, and WN1, only had around one month of data measured over this period. The remaining bathrooms had data measured throughout this period. The eight 
houses without a complete set of data from this period have not been included in the full analysis. This leaving 20 houses with 24 bathrooms.

The results of the full analysis were designed to better understand the performance of individual bathrooms and to provide some indication of trends between houses over the selected period. Restricting the analysis to this period ensured as many of the factors that may influence results are kept as consistent as possible.

While the results of a full analysis may not be able to be directly compared, the overall performance of these houses at any point in time during the year would be a useful result to record. These factors could then be used to understand how each home may perform during the rest of the year. For example, a house measured from August to January may perform well during throughout this period, effectively removing moisture during this time. As August is the only winter month recorded, there may be insufficient information to show how well this house may typically perform in winter. However, if the house is shown to perform similarly to a house running from May to September during the months which contained measurements from both houses, then you may assume that the house would perform similarly at other times of the year. These would be very basic assumptions that would simply provide an indication of which houses may be most at risk. Data from the indicated periods would need to be gathered before any analysis of these buildings could take place. These types of assumptions are beyond the scope of this research.

It is also important to note that while using the same two-month period for all houses does mitigate some of the issues, there is no way to completely account for any differences between houses. For example, these measurements were taken from throughout New Zealand in seven different areas. Although the measurement period is the same, the weather varied depending on where in the country the house was located.

\subsection{Data Available for Selected Houses}

\begin{tabular}{|l|l|l|l|l|l|l|l|l|}
\hline \multicolumn{1}{|c|}{ Region } & \multicolumn{1}{|c|}{ Simplified ID } & RH & T & W & Vent & Area & Date & Time \\
\hline \multirow{5}{*}{ Dunedin } & DN1 & & & & & & & \\
\cline { 2 - 8 } & DN2 & & & & & & \\
\cline { 2 - 8 } & DN4 & & & & & \\
\cline { 2 - 8 } & DN6.1 & & & & & \\
\cline { 2 - 8 } & DN6.2 & & & & & & \\
\hline \multirow{5}{*}{ Wellington } & WN2 & & & & & & & \\
\cline { 2 - 8 } & WN3 & & & & & \\
\hline
\end{tabular}

95 


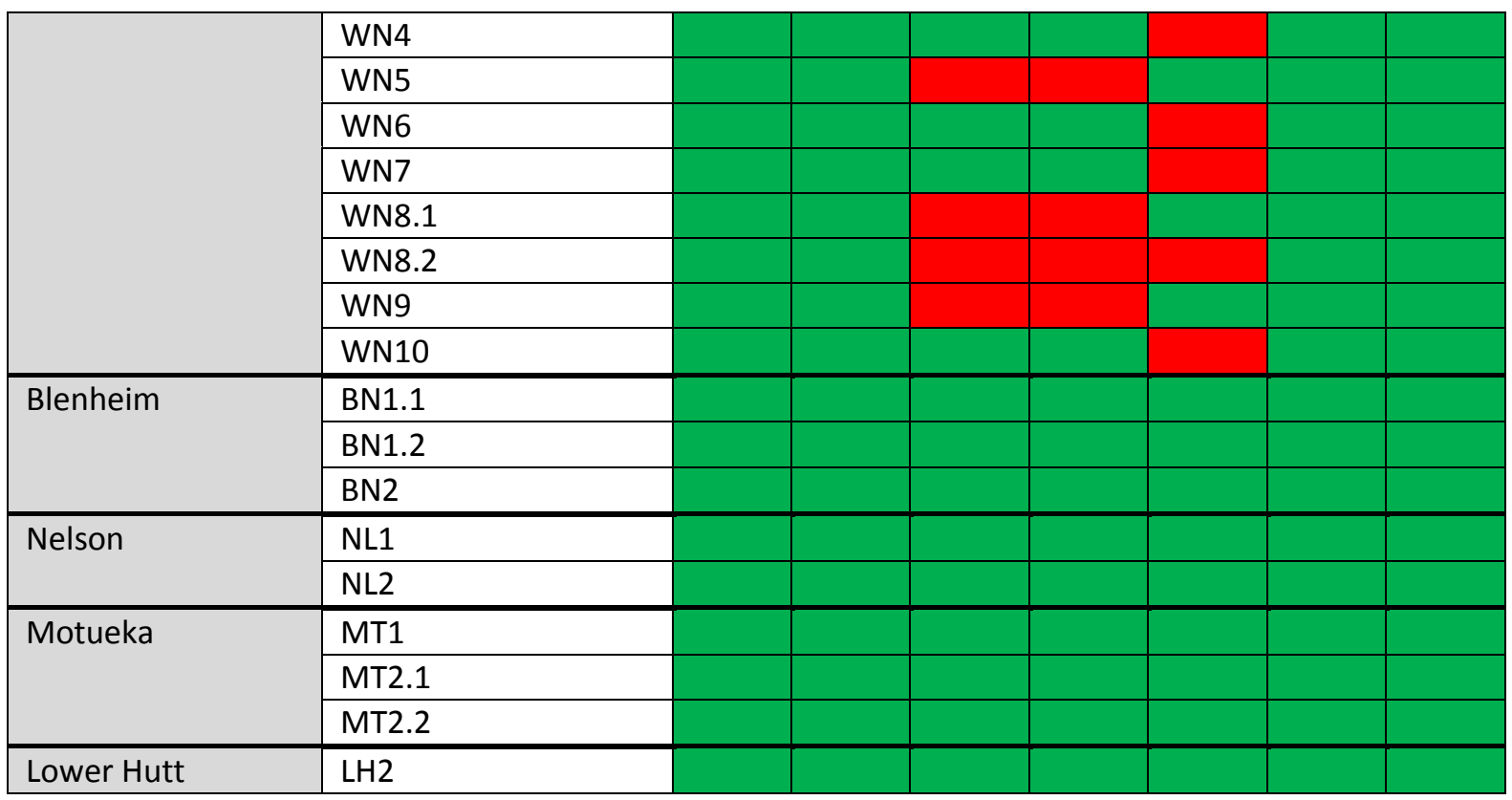

Table 13 Available Data for Selected Houses

\begin{tabular}{|l|l|l|}
\hline Colour/Column & Example & \multicolumn{1}{c|}{ Meaning } \\
\hline $\begin{array}{l}\text { Red } \\
\text { Available Data }\end{array}$ & & The data is not available for this house. \\
\hline $\begin{array}{l}\text { Green } \\
\text { Available Data }\end{array}$ & & The data is available for this house. \\
\hline
\end{tabular}

Table 14 'Available Data for Selected Houses' Key

Table 13 provides a summary of the data available for further analysis. Processing the data required information on the relative humidity $(R H)$, temperature $(T)$, date, and time.

Information on the presence of openable windows (W), the presence of mechanical ventilation (Vent), and the room dimensions (Area) are not required for processing the measurements and identifying moisture events, but are useful for analysing and interpreting the results.

\subsection{Results}

\subsubsection{Data Processing}

The selected houses were processed using the template described in 6.5.4 Creating a Data Analysis Template. This calculated the absolute humidity for every measurement, sorted the data into five minute periods, and displayed the results for each day in a graph. This provided the information necessary to define each type of moisture event, as described in 0 
Moisture in New Zealand Bathrooms

Defining Categories of Moisture Events.

\subsubsection{Calculating Average Moisture Events}

To perform a quantitative analysis on the moisture events in each house, more information was required. This included the length of each moisture event as well as the absolute humidity of the start, peak, and end of each moisture event.

\subsubsection{Data for Analysis}

The processed measurements from 7.3.1 Data Processing were used for these calculations. This included the original measurement times, before they were broken into five minute periods. This ensured that the moisture event length was as accurate as possible. All measurements that did not register as a moisture event were removed. All moisture events which took place outside of the August/September period, as defined in 7.1.3 Selecting Analysis Period, were removed.

\subsubsection{Selecting Appropriate Moisture Events for Analysis}

Some moisture events did not contain a start, peak, and end. For example, the absolute humidity may increase or decrease to a certain level (at a rate greater than $3 \%$ ) and remain at that level. In this moisture event, the peak and end are the same. All moisture events that did not contain an increase followed by a decrease were removed.

The remaining moisture events were classified as episodes, following the general definition provided in 7.4.1 Episode. This version of episodes included every series of measurements which involved an increase followed by a decrease, regardless of context. This was different to the episodes included in typical days, which filtered episodes based on size and length as defined in 7.6.1 Filtering Episode.

\subsubsection{Finding Mean and Median Length}

The length of time between the start and end of all remaining moisture events was calculated. All results that took place over a period of more than three hours were checked for accuracy using the graphs produced by 7.3.1 Data Processing. In some rare cases a gap in the measurements was present, resulting in a false moisture event. These results were removed. The mean, minimum, first quartile, median, third quartile, and maximum were calculated to the nearest minute for the remaining results. Table 15 shows an example of the results.

97

Full-Scale Data Processing 


\begin{tabular}{|l|c|c|}
\hline \multicolumn{1}{|c|}{ House: DN1 } & Time (Seconds) & Time (Minutes) \\
\hline Mean & 5200 & 87 \\
\hline Standard Deviation & 3148 & 52 \\
\hline & & 15 \\
\hline Minimum & 891 & 31 \\
\hline Q1 & 1879 & 87 \\
\hline Median & 5246 & 123 \\
\hline Q3 & 7397 & 232 \\
\hline Maximum & 13912 & \\
\hline
\end{tabular}

Table 15 DN1, Example of Mean and Median Moisture Event Lengths

\subsubsection{Finding Mean and Median Absolute Humidity at Start, Peak, and End}

The mean, minimum, first quartile, median, third quartile, and maximum of the absolute humidity at the start, peak, and end of every moisture event were calculated. This included all peaks that occurred during each moisture event. Table 16 shows an example of the result.

\begin{tabular}{|l|c|c|c|}
\hline \multicolumn{1}{|c|}{ House: DN1 } & Start $\left(\mathrm{g} / \mathrm{m}^{3}\right)$ & Peak $\left(\mathrm{g} / \mathrm{m}^{3}\right)$ & End $\left(\mathrm{g} / \mathrm{m}^{3}\right)$ \\
\hline Mean & 7.61 & 10.43 & 8.20 \\
\hline Standard Deviation & 1.29 & 3.91 & 1.42 \\
\hline & & & 5.16 \\
\hline Minimum & 4.63 & 5.16 & 7.13 \\
\hline Q1 & 6.65 & 7.49 & 8.01 \\
\hline Median & 7.58 & 9.01 & 9.12 \\
\hline Q3 & 8.64 & 12.99 & 12.78 \\
\hline Maximum & 11.14 & 21.00 & \\
\hline
\end{tabular}

Table 16 DN1, Example of Mean and Median Absolute Humidity at Each Point of Moisture Events 


\subsection{Defining Categories of Moisture Events}

This chapter defines the different types of moisture event used to group similar series of measurements. The categories used were:

- episode;

- increase;

- decrease; and

- combination.

The 'episode' category was the main tool used to analyse the indoor environment. Days which share similarities in the frequency of episodes taking place can be compared and analysed. The other three categories are included for three reasons:

- To provide context for the episode. For example, if a decrease is recorded for a period immediately after an episode is recorded, this may provide additional information on how that particular house is ventilated and could be worth comparing to other houses where these same conditions were recorded.

- To ensure no episodes were missed. In some situations a house may introduce or remove moisture in a way that differs from other houses. In these situations, any type of change in absolute humidity would help identify these moisture events. For example, if a shower includes a shower dome, moisture may be introduced to the main bathroom space at a slower rate (van Dijk \& Carson, 2011). This could potentially appear as an increase rather than an episode. This makes it important to note how frequently these different types of categories occur.

- To give better insight into how bathrooms are performing. While not the main focus of this study, taking note of patterns in how absolute humidity changes in different houses provides additional information that results can be compared to. This can help demonstrate what impact moisture events may have on the rate of change of absolute humidity throughout the day.

\subsubsection{Episode}

This is the type of change the process carried out in Chapter 7: Full-Scale Data Processing was primarily attempting to identify. An 'episode' consisted of the absolute humidity rapidly increasing before reaching a peak and beginning to decrease. The main criteria for a 
moisture event to be classified as an episode was for there to be a positive rate of change at the start, reaching a peak and then decreasing. In some cases there were multiple increases and decreases taking place during a moisture event, forming multiple peaks.

\subsubsection{Increase}

An 'increase' was defined as a moisture event where the absolute humidity maintained a positive rate of change over the full series of measurements. Unlike an episode, an increase ended once it reached its peak. An increase indicated that the absolute humidity remained at this increased level, decreasing (or potentially continuing to increase) at a rate of less than $3 \%$ between each measurement.

\subsubsection{Decrease}

A 'decrease' was defined as a moisture event where the absolute humidity maintained a negative rate of change over the full series of measurements. Unlike an episode, a decrease started at its peak. A decrease indicated that the absolute humidity began did not experience any large changes before the decrease began, with any change taking place at a rate of less than $3 \%$ between each measurement.

\subsubsection{Combination}

Moisture events that did not meet the criteria of episodes, increases, or decreases were classified as 'combinations'. Examples of combinations include moisture events where the absolute humidity decreased, reached a minimum point, and then increased.

\subsection{Filtering Moisture Events for Typical Days}

To identify trends, common occurrences, and outliers, each day was listed as involving a certain pattern of events, allowing similar days to be compared. This was done using points identified as moisture events in Chapter 7: Full-Scale Data Processing.

These moisture events needed to be further filtered so that only the moisture events most likely to have an impact on the indoor environment (and form patterns between each day) were included. To qualify as an episode, increase, or decrease for a typical day, a series of measurements qualifying as a moisture event must either:

- be spread over a period of more than 45 minutes where no more than half of the measurements were potential moisture events; or 
- at any point, the absolute humidity reaches $1.3 x$ or $0.7 x$ the absolute humidity at the start of the series.

Each typical day was broken into six periods:

- 12.00am-4.00am;

- 4.00am-8.00am;

- 8.00am-12.00pm;

- $12.00 \mathrm{pm}-4.00 \mathrm{pm}$;

- $4.00 p m-8.00 p m ;$ and

- $8.00 \mathrm{pm}-12.00 \mathrm{am}$.

Each period listed the type of moisture event taking place in it, as determined by the additional criteria described in 7.6 Changes in Definitions for Typical Days. These restrictions were designed to consistently identify periods of change which had a large impact on the indoor environment. While small changes over short periods still contributed towards the indoor environment, these were excluded from filtered moisture events.

\subsection{Changes in Definitions for Typical Days}

\subsubsection{Filtering Episodes}

Additional considerations for identifying episodes for typical days included:

- when the episode took place over two or more four-hour periods, the event would be recorded as taking place during the period the peak occurs in (Figure 48);

- when the peak occurred within 5 minutes either side of the start/end of a period (e.g., 7.55am - 8.05am) the episode was recorded as taking place during whichever of these two periods contain more measurements identified as being part of the series; and

- when measurements qualifying as an episode had two or more peaks, each in a different four-hour period, all periods containing a peak were marked as containing an episode (Figure 49).

The occurrence of an episode took precedence over any other type of change that may occur within the same four-hour block. For example, if an increase and a decrease both occur between $8.00 \mathrm{am}$ and $12.00 \mathrm{pm}$, that period would be listed as containing an episode. 


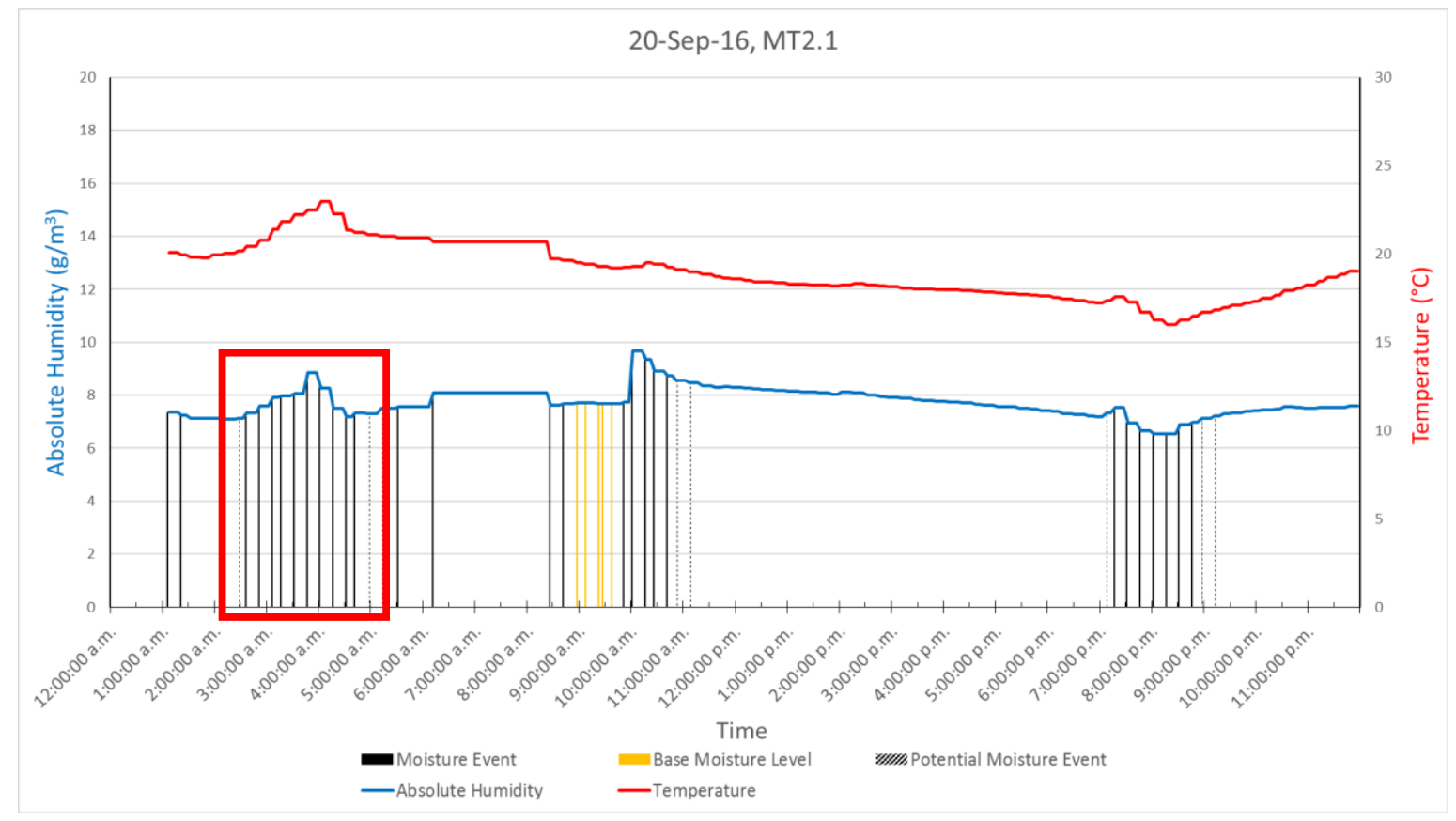

Figure 48 Example of Episode: One Peak

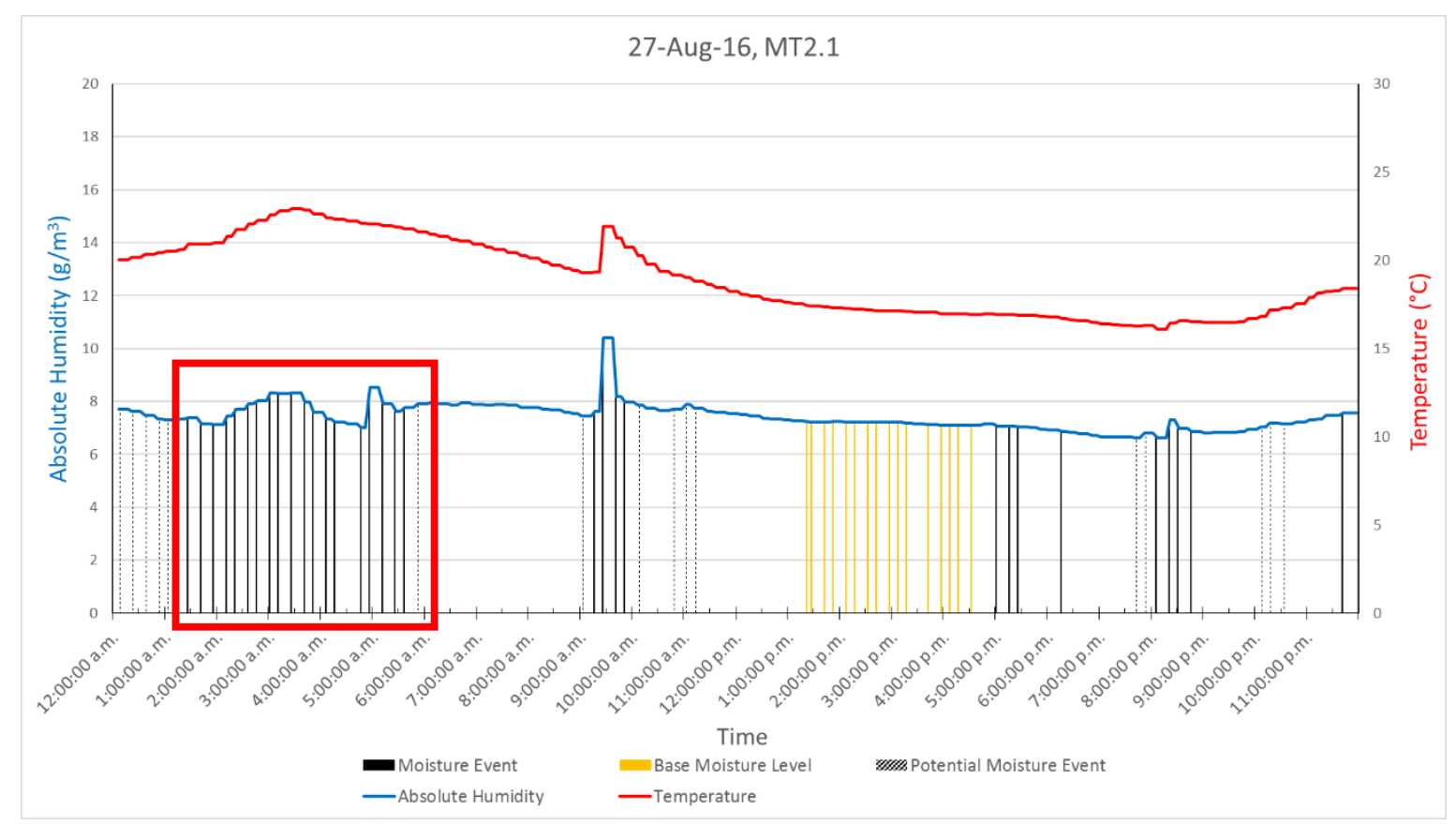

Figure 49 Example of Episode: Two Peaks

\subsubsection{Filtering Increases}

An increase is differentiated from an episode when the absolute humidity:

- increases at a constant rate (Figure 50); and 
- is not immediately followed by a decrease that may be interpreted as part of the same series of measurements.

The increase is classified as taking place in the period containing the greatest number of measurements identified as part of the series. The constant rate requirement is designed to further differentiate an increase from the beginning of an episode.

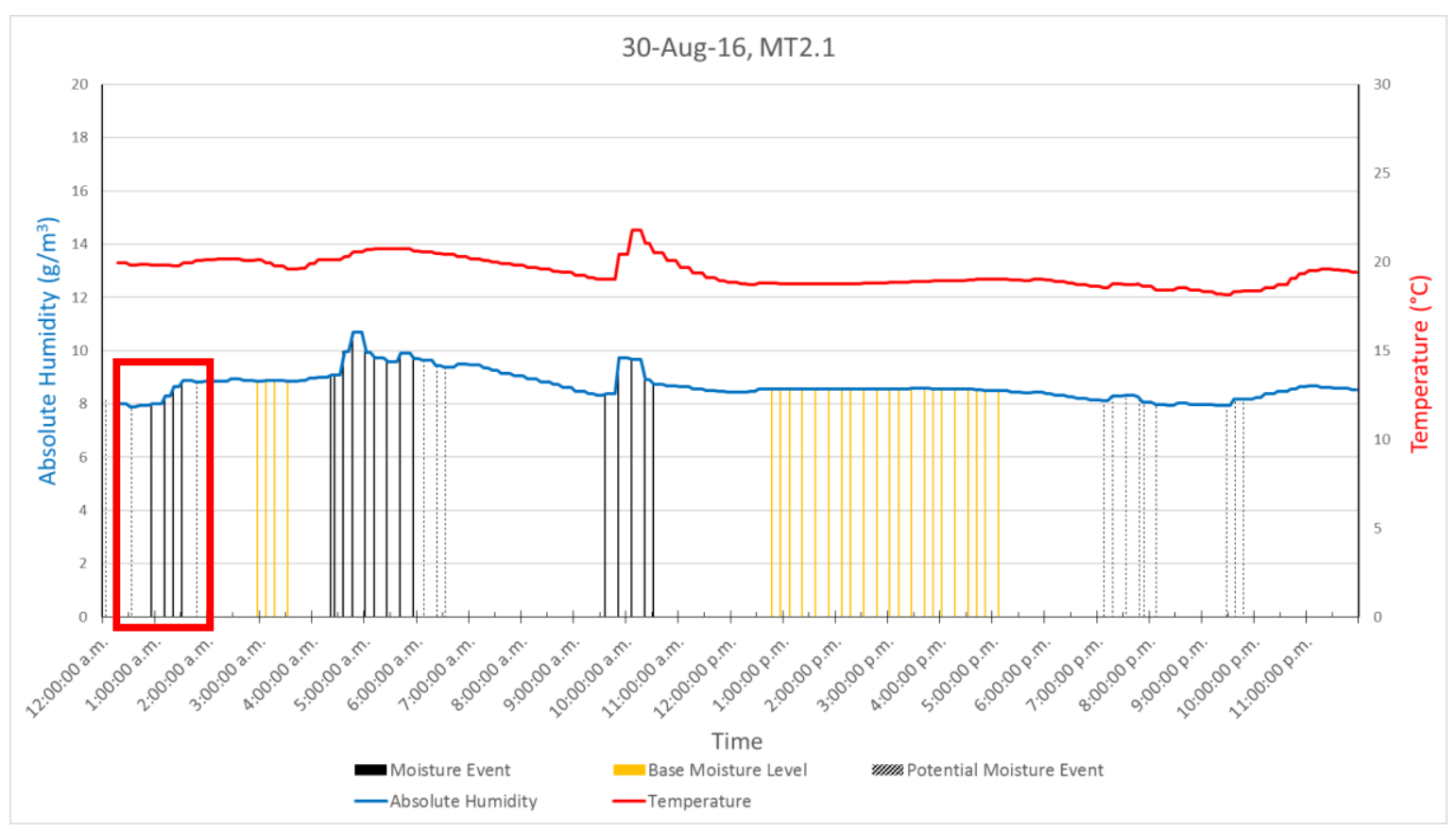

Figure 50 Example of Increase

\subsubsection{Filtering Decreases}

A decrease is differentiated from an episode when the absolute humidity:

- Decreases at a constant rate; AND

- Is not immediately preceded by a decrease that may be interpreted as part of the same series of measurements.

The decrease is classified as taking place in the four-hour period containing the greatest number of measurements identified as part of the series. For example, in Figure 51 the 12.00am - 4.00am period would be counted as empty while the 4.00am - 8.00am period would be counted as containing the decrease. 


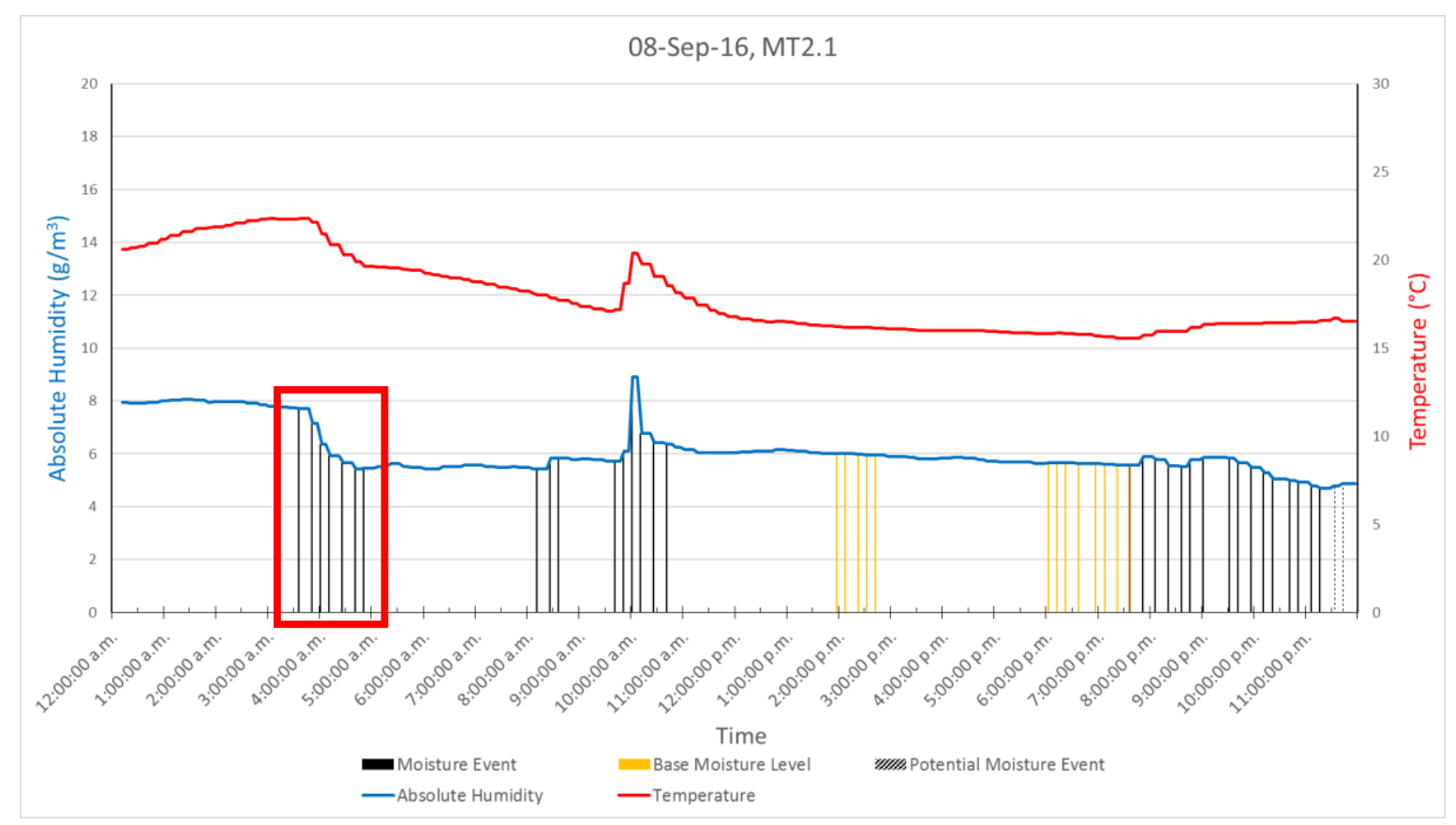

Figure 51 Example of Decrease

\subsubsection{Combinations for Typical Days}

Moisture events that met the criteria for typical days but did not fit into any other category were classed as 'combinations'. The may be recorded where an increase and a decrease (as two distinct series of measurements) occur within the same period. It also includes unusual combinations of readings that should be further analysed. For example, a situation where there is a single series of measurements involving a decrease and subsequent increase of absolute humidity, appearing as a 'reverse episode' ( 
Moisture in New Zealand Bathrooms

\section{Figure 52 Example of Combination}

).

07-Aug-16, MT2.1

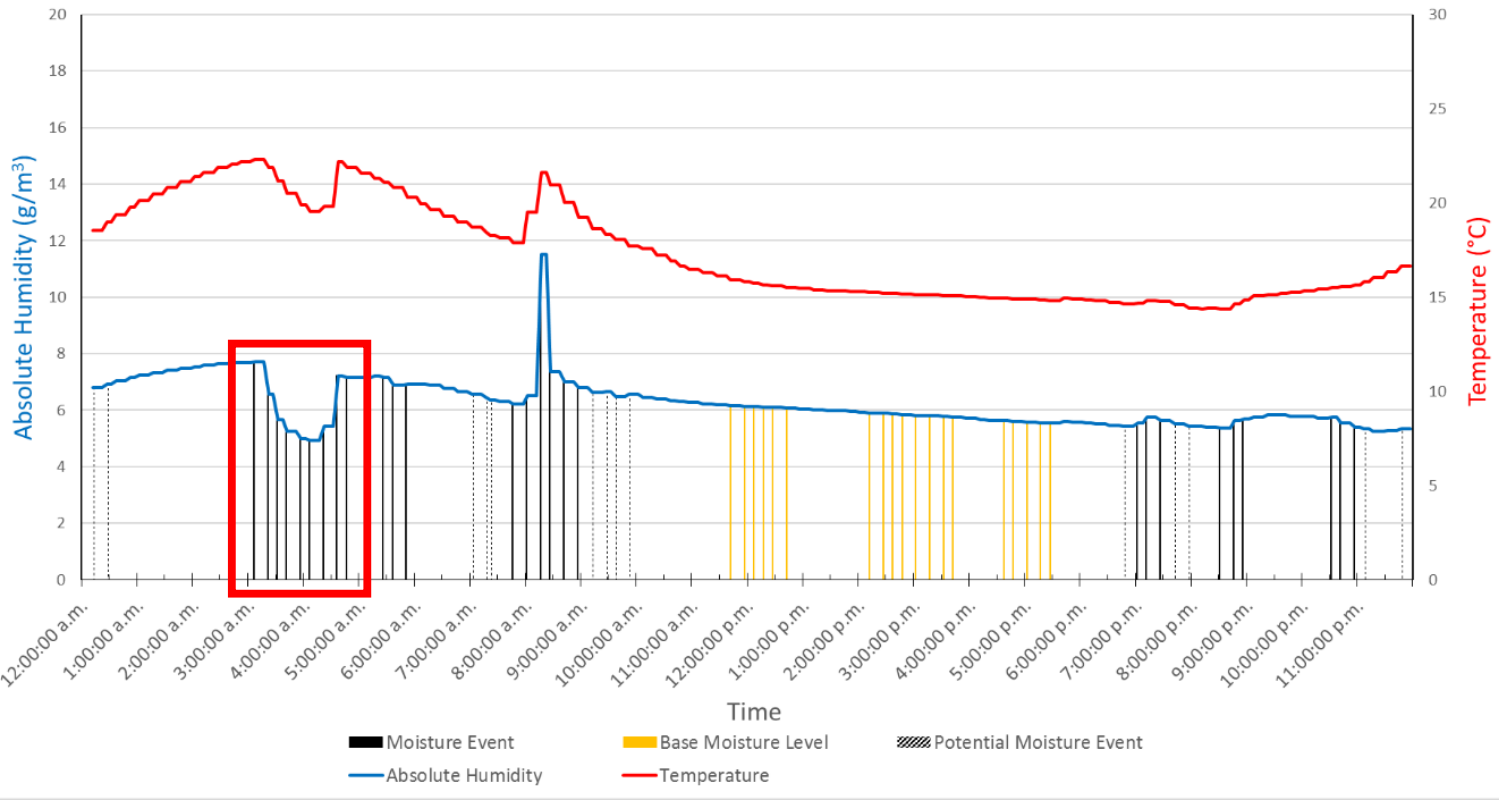


Alister Stubbe

Figure 52 Example of Combination 


\section{Chapter 8: Typical Days}

\subsection{Typical Day Process}

\subsubsection{Defining Typical Days}

Moisture events caused by a source of moisture regularly used in a similar way each day (such as occupants taking a shower each morning) may result in a consistent amount of moisture being introduced to the indoor environment at similar times. Days where similar trends occurred can be classified as being a single type of day. The types of days which occurred most frequently were referred to as 'typical days' and could be used to take a representative sample of measurements from each house.

\subsubsection{Summarising Data}

With the data processed, moisture events that meet the set of specified criteria described in 7.6.1 Filtering Episodes were sorted based on the rate of change and when they occurred. This was completed to identify common trends in each house.

Typical days could then be selected. Further analysis of the house the data is taken from can be made considering what type of typical day was most common. For example, there may be 20 instances of one type of typical day where no episodes took place, 15 instances of a second type of typical day where one episode took place, and 10 instances of a third type of typical day where no moisture events took place, all over the 61 day period (August and September). In this situation, 45/61 days were represented. An analysis of the episodes that took place over the entire period could then be stated to represent a total of between 15 and 31 days.

This also allows different types of days to be compared. For example, 'typical day 1' may consist of a moisture event in the morning and at night while 'typical day 2 ' may only consist of a moisture event at night. The impact the morning event has on the indoor environment throughout the day can then be better understood by comparing how the night moisture event changes. This is beyond the scope of this study but should be considered for future research. 


\subsubsection{Sorting Typical Days}

Once each moisture event has been categorised for all days during the selected months, the days can be grouped based on similarities in results. This was primarily based on the type of change present (episode, increase, decrease, or combination), the number of times those changes took place, and the length of time between those changes. While the exact time each episode took place does make a difference, for example when considering the impact of temperature differences between day and night, but these differences could be accounted for by calculating the impact of external conditions. This is beyond the scope of this study. At least three examples of any one type of day ( $5 \%$ of total days) was required for that type of day to be considered a typical day.

\subsection{Example of Categorising Moisture Events: DN1}

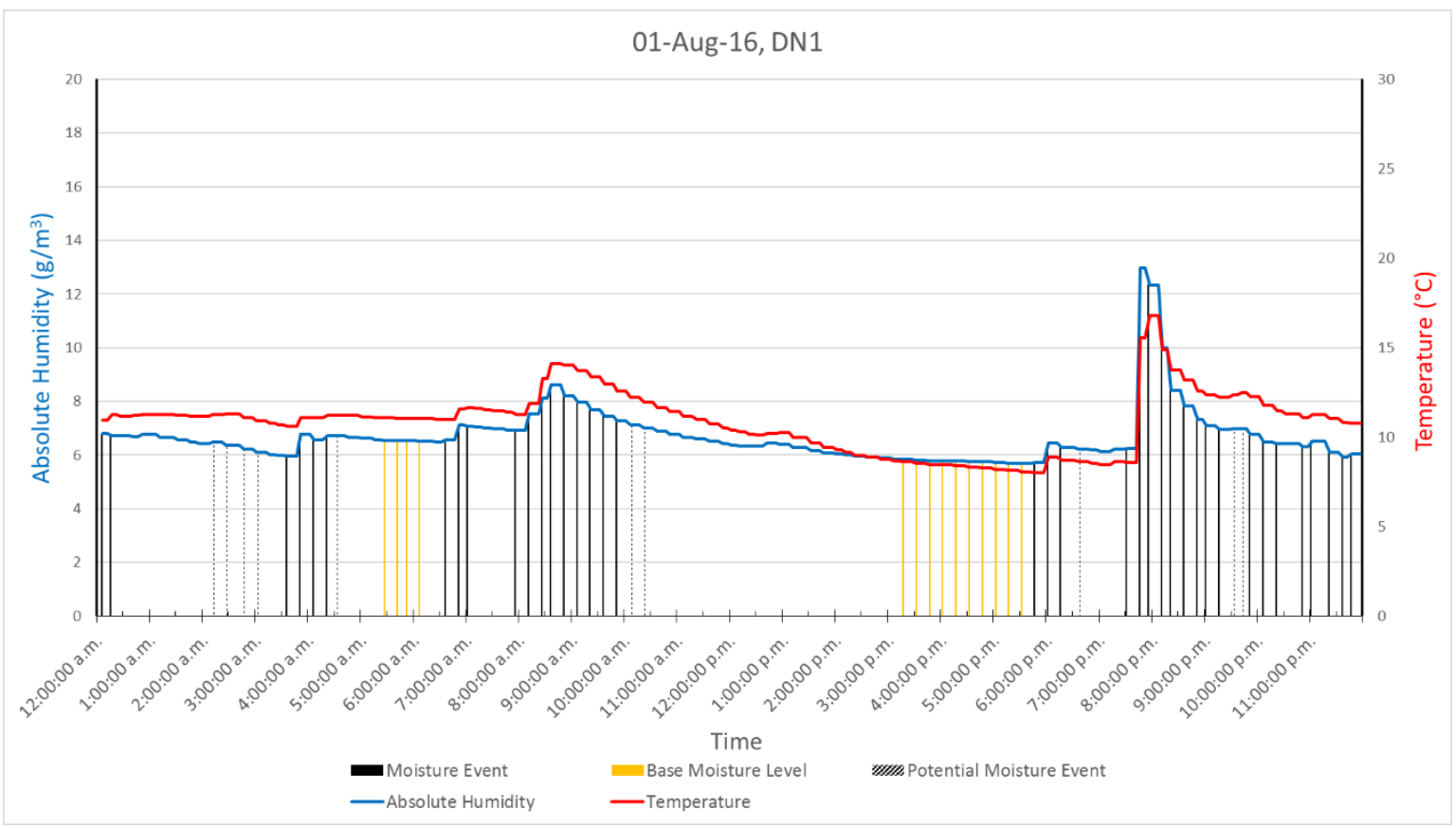

Figure 53 DN1, Monday - 01/08/16

Figure 53 shows data recorded on 01/08/16 in house DN1. The process for identifying moisture event categories looks at each period individually.

- 12.00am - 4.00am: While the very first measurements are recorded as moisture events, the series did not last long enough (or reach high enough levels of absolute 
humidity) to count towards the typical day. A series of potential moisture events began at $2.15 \mathrm{am}$. However, as no measurements in the series classify as a moisture event, these were ignored. A moisture event began at the end of the period but the peak occurs after 4.00am. This period was recorded as blank.

- 4.00am - 8.00am: The peak of a moisture event occurred at the very start of the period. The moisture event took place over a period of more than 45 minutes and consisted of an increase followed by a decrease. This period was recorded as containing an episode.

- 8.00am - 12.00pm: The peak of a moisture event occurred before 9.00am. The moisture event took place over a period of more than 45 minutes and consists of an increase followed by a decrease. This period was recorded as an episode.

- $12.00 \mathrm{pm}-4.00 \mathrm{pm}$ : No moisture event took place within this period. This period was recorded as blank.

- $4.00 \mathrm{pm}-8.00 \mathrm{pm}$ : A moisture event occurred at $6.00 \mathrm{pm}$ that, including one potential event, took place over a period of more than 45 minutes. The peak of a moisture event occurs before $8.00 \mathrm{pm}$. The moisture event took place over a period of more than 45 minutes and consisted of an increase followed by a decrease. This period was recorded as containing an episode.

- $8.00 \mathrm{pm}-12.00 \mathrm{am}$ : Although a moisture event took place throughout this period, this was already recorded as taking place during the previous period. A second series of measurements recorded as a moisture event appears to have begun just before $10.00 \mathrm{pm}$. Looking at the potential moisture events taking place before this series show that it was part of the same series of measurements as the previous moisture event. This period was recorded as blank. 


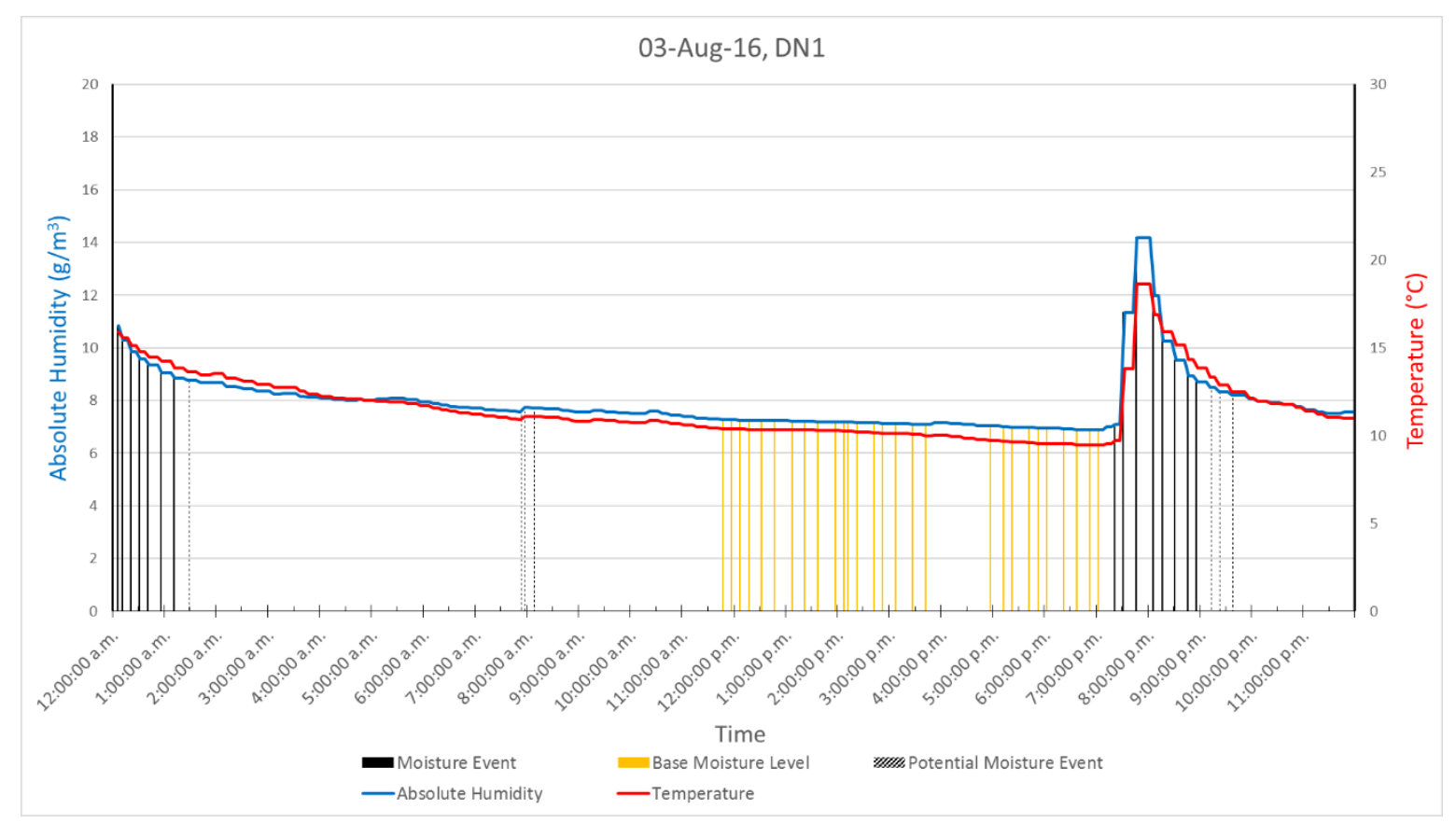

Figure 54 DN1, Tuesday - 02/08/16

Figure 54 shows data recorded on 03/08/16 in house DN1

- 12.00am - 4.00am: A series of measurements from 12.00am until just after 1.00am register as a moisture event. This period was recorded as a decrease.

- 4.00am -8.00am: No moisture event took place within this period. The period was recorded as blank.

- $8.00 \mathrm{am}-12.00 \mathrm{pm}$ : No moisture event took place within this period. The period was recorded as blank.

- $12.00 \mathrm{pm}-4.00 \mathrm{pm}$ : No moisture event took place within this period. The period was recorded as blank.

- $4.00 \mathrm{pm}-8.00 \mathrm{pm}$ : The peak of a moisture event occurred before 8.00pm. The moisture event took place over a period of more than 45 minutes and consisted of an increase followed by a decrease. This period was recorded as containing an episode.

- $8.00 \mathrm{pm}-12.00 \mathrm{am}$ : Although a moisture event took place throughout this period, this was already recorded as taking place during the previous period. This period was recorded as blank. 


\subsection{Example of Typical Day Summary: DN1}

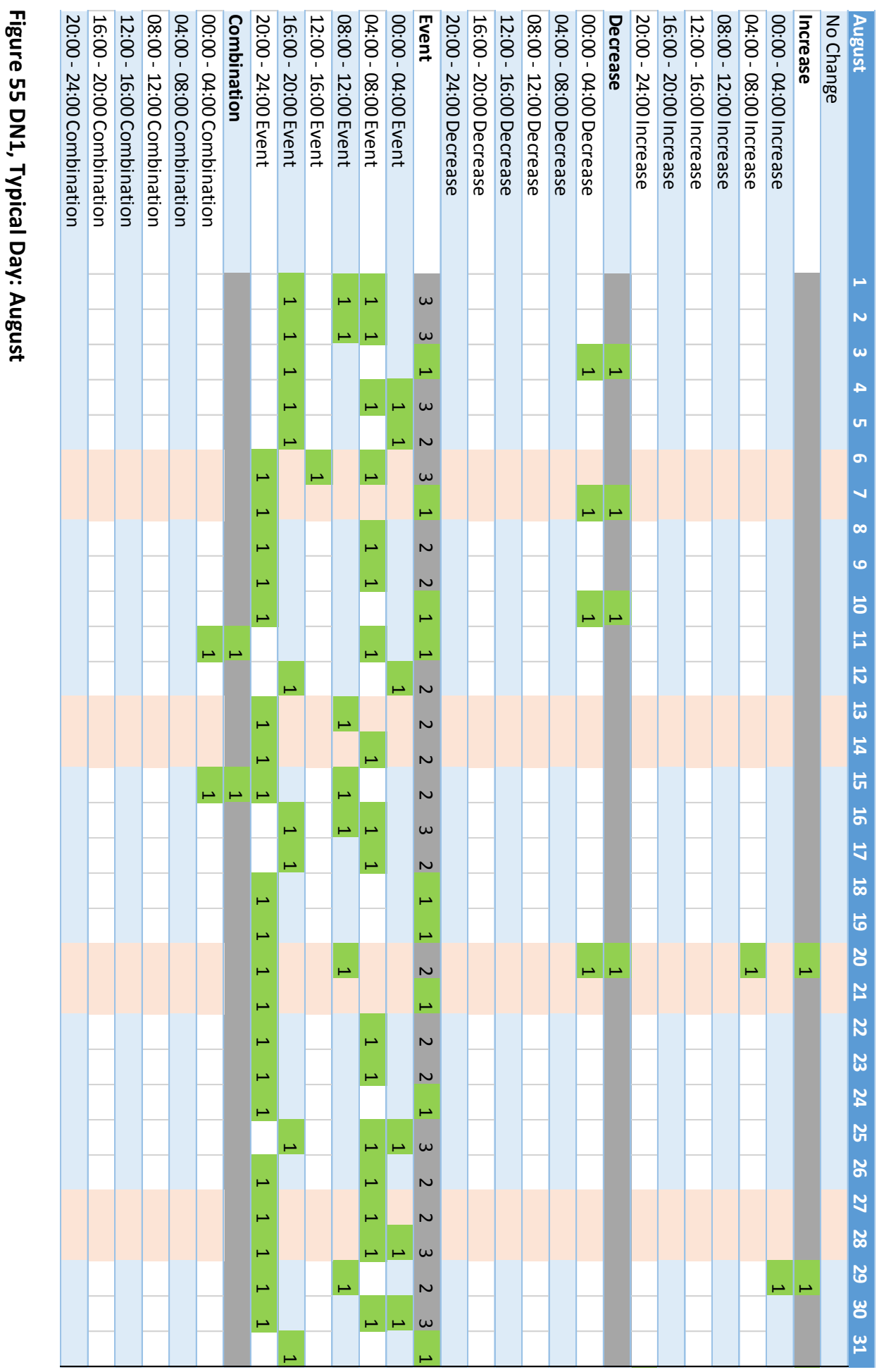




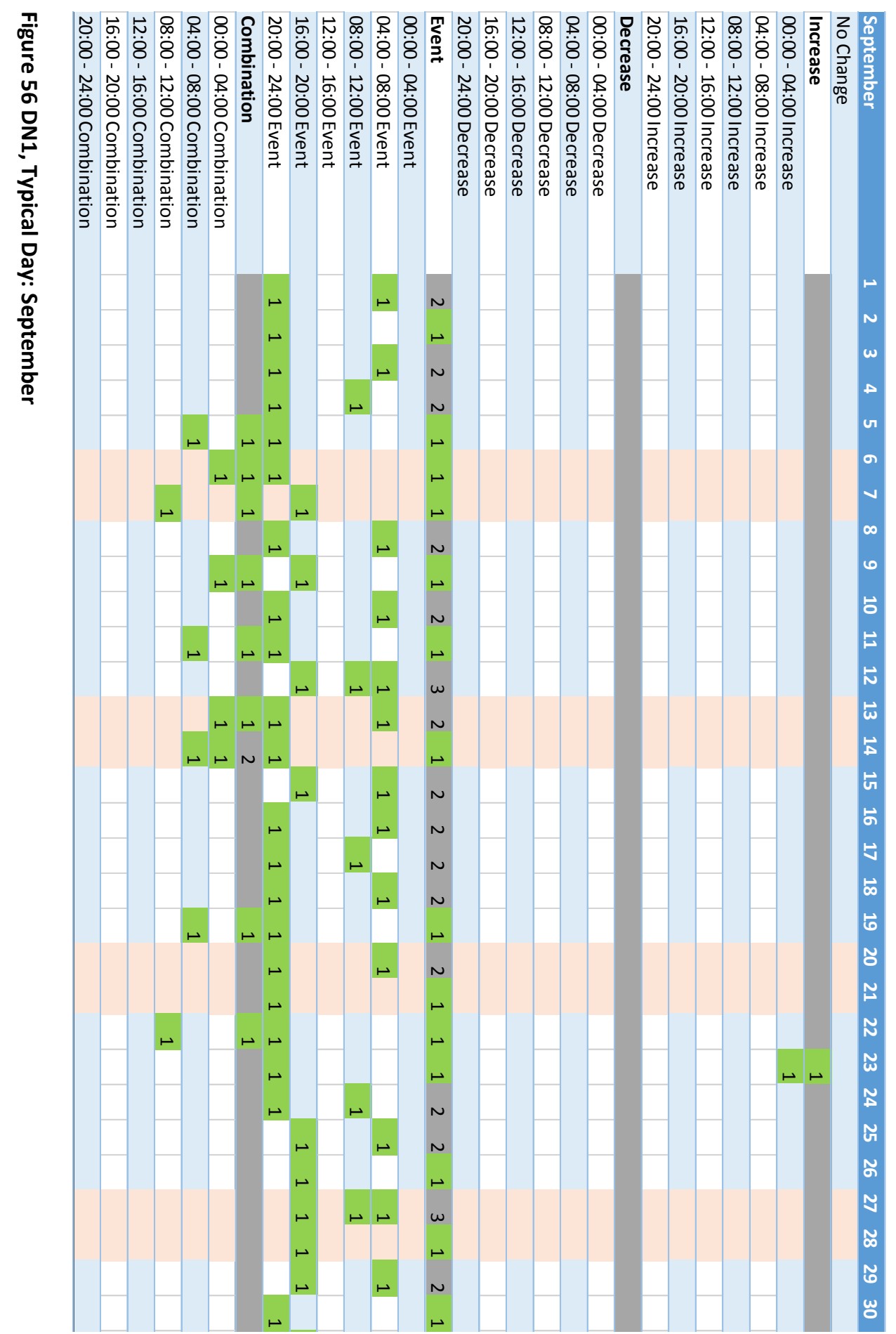




\subsection{Example of Typical Day Summary: 2 Bathrooms}

\subsubsection{Typical Days: BN1.1}

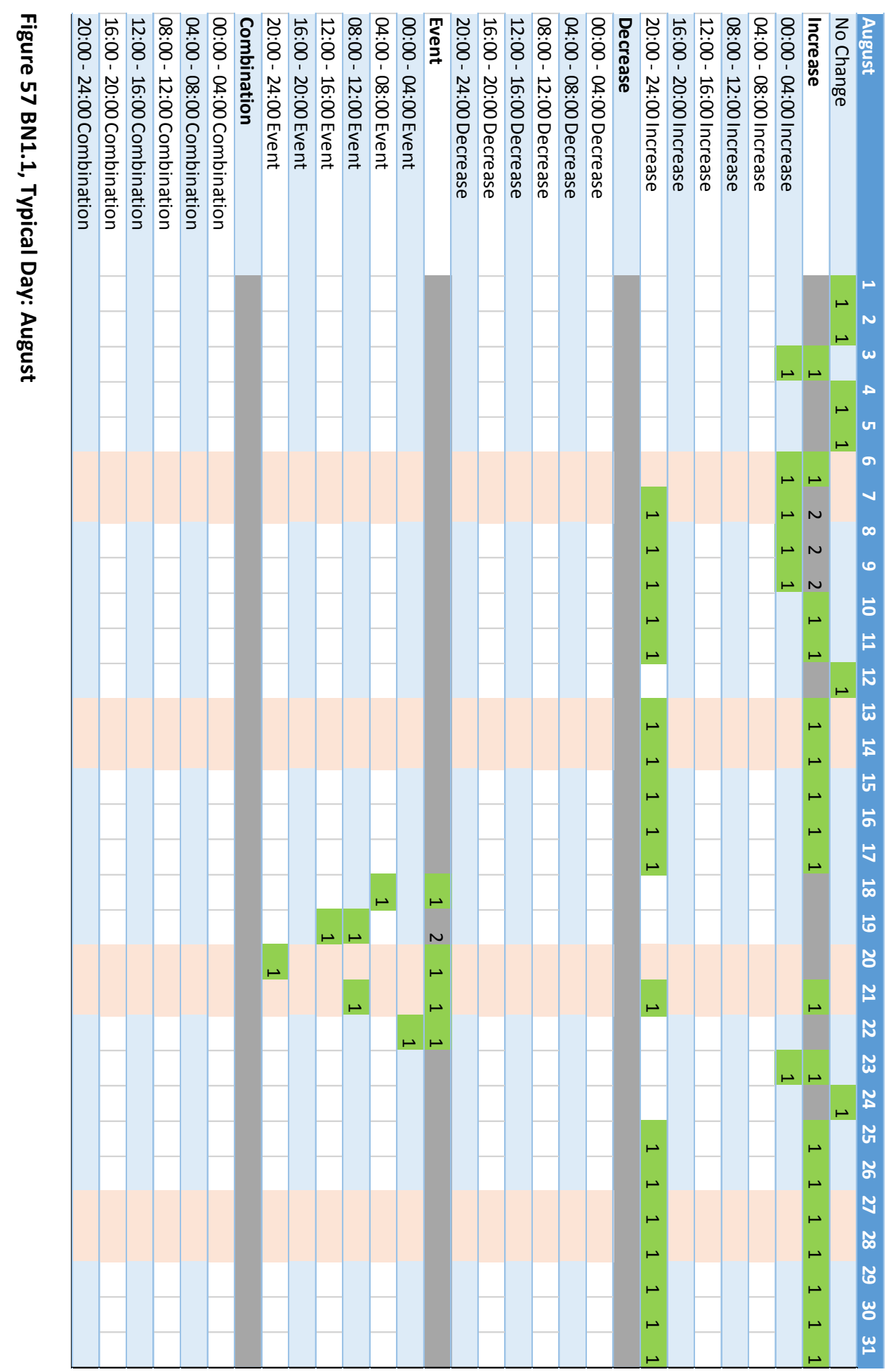




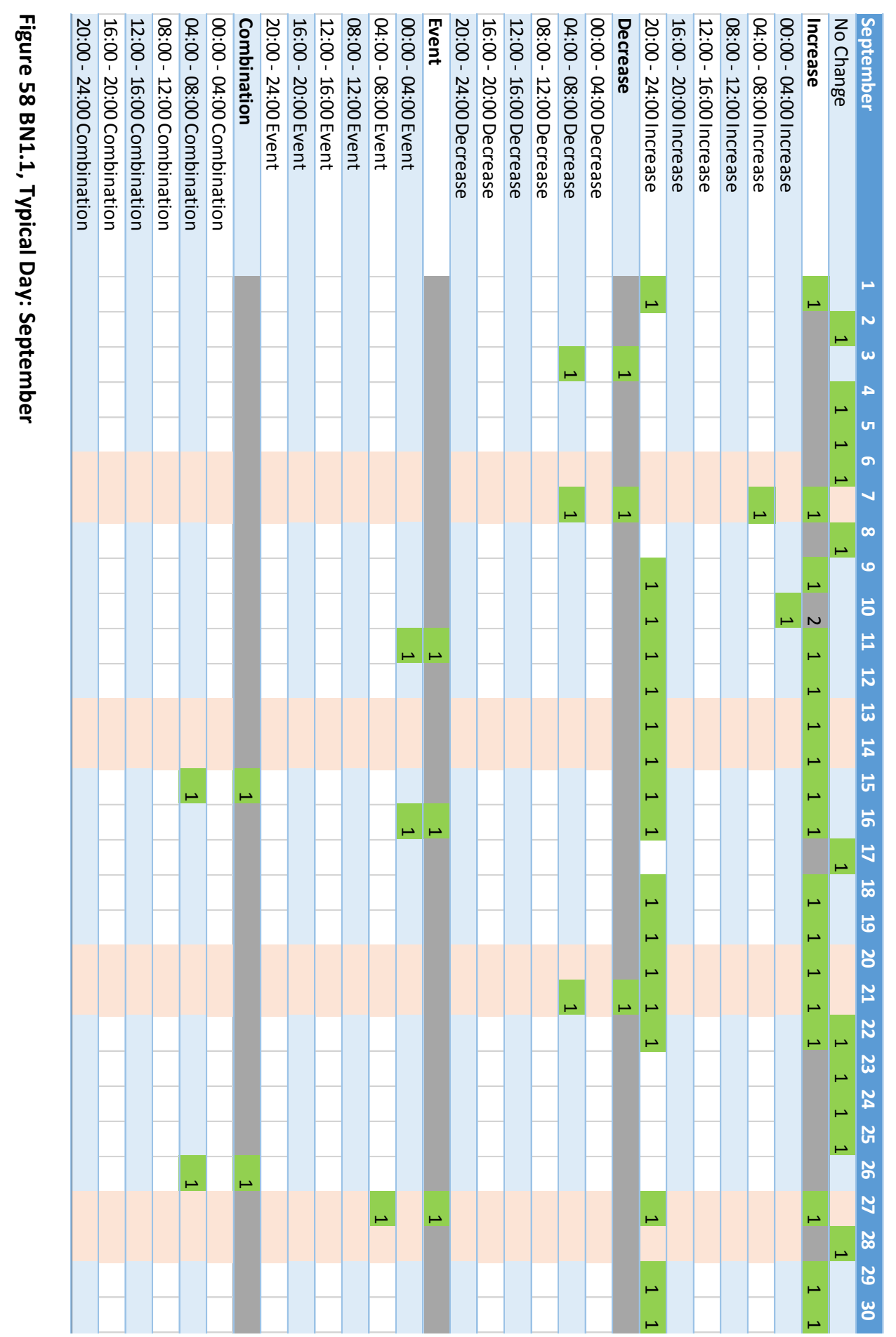




\subsubsection{Typical Days: BN1.2}

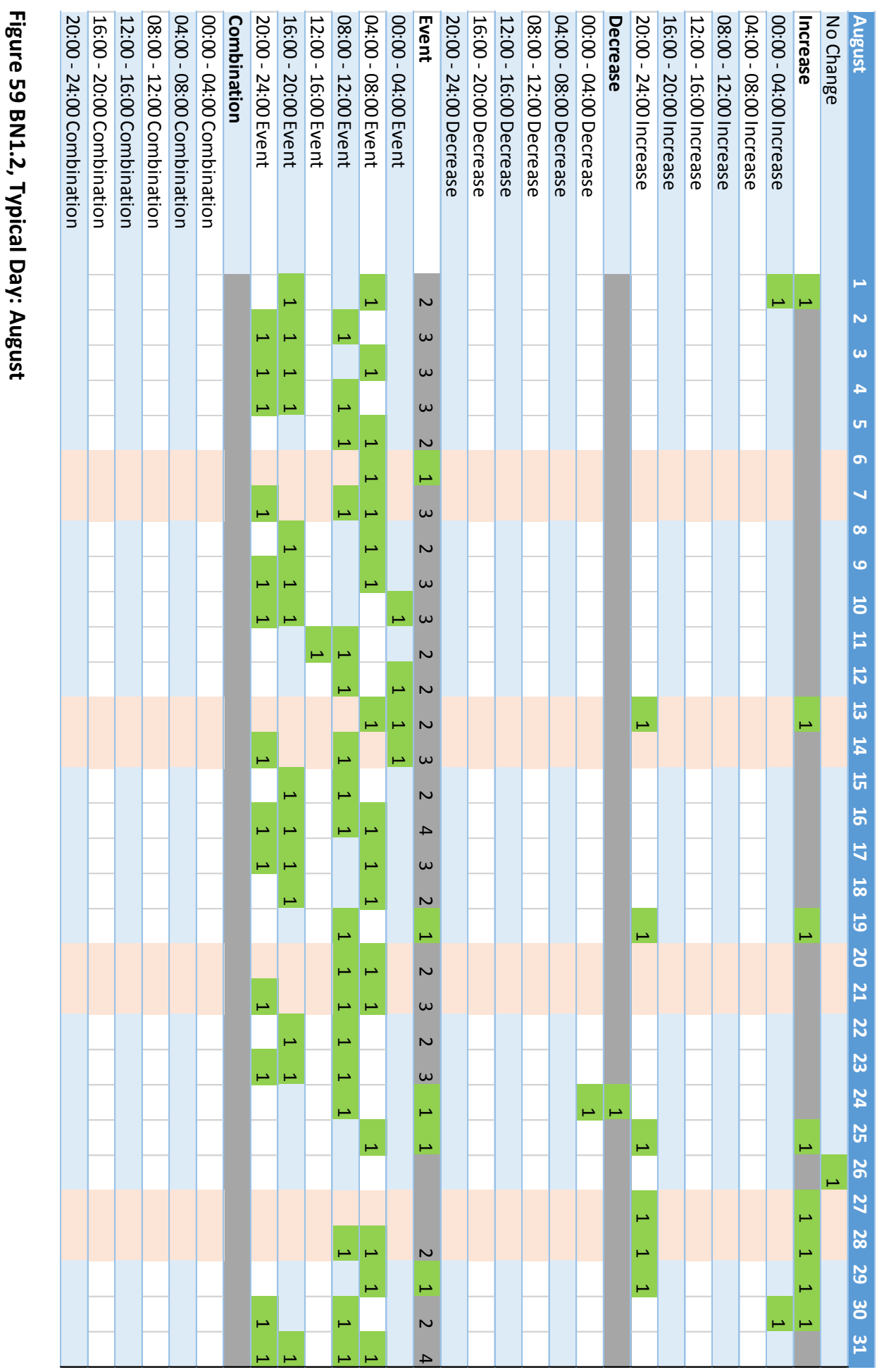




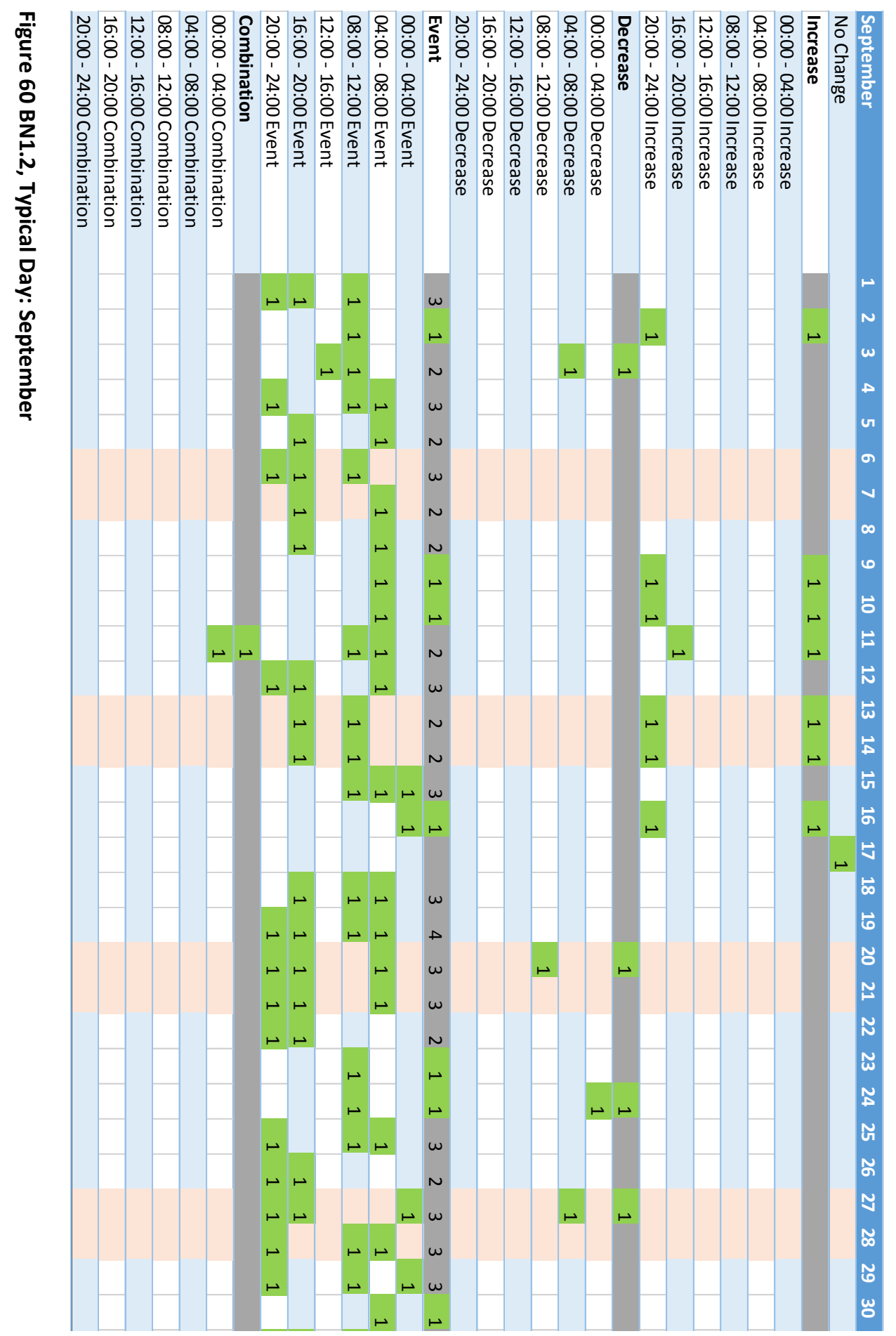




\subsection{Example of Producing Typical Days: BN1.1}

The first bathroom of house BN1 contained very few moisture events identified as episodes. Instead, the main category recorded were increases. Out of the 61 days analysed, an increase took place in 39 (64\%) of them. A total of 43 increases took place over the entire 61 day period. Seventeen days (28\%) contained no moisture events. Twenty-four days (39\%) involved only a single increase, while four more days (66\%) involved only two increases. The vast majority of increases took place between $8.00 \mathrm{pm}$ and $12.00 \mathrm{am}$, with 35/43 increases taking place within this period. On days where an increased occurred between $8.00 \mathrm{pm}-$ 12.00am and an increase occurred the next day from 12.00am -4.00am, these two recordings were often part of the same series of measurements. Out of the 11 days with a morning increase, seven followed an increase at the end of the previous day. Three out of the remaining four days had "potential moisture events" in the same series (also increasing) take place the night before. Only the increase recorded on 6/08/2016 had no increase or potential increase take place during the previous period.

Only eight days (13\%) involved an episode. Five of these six days were consecutive, from $18 / 08 / 2016$ to $22 / 08 / 2016$. Only two days involving an episode also involved an increase, one of these being the non-consecutive day. The time episodes took place at was inconsistent. The only period where an episode did not take place was between $4.00 \mathrm{pm}$ and 8.00pm; every other period contained either one or two episodes.

Seven days (11\%) involved a decrease, all of which took place in September. Three of these days were consecutive, from 20/09/2016 to 22/09/2016. Every day with a decrease other than 3/09/2016 also had an increase take place. Every decrease took place between 4.00am and 8.00am.

These results can be grouped into five typical days representing BN1.1:

- days where no moisture event took place (Figure 61);

- days where an increase took place late at night (Figure 62);

- days where an increase took place in the early morning (Figure 63); and

- days where an episode took place (Figure 64). 
12-Aug-16, BN1

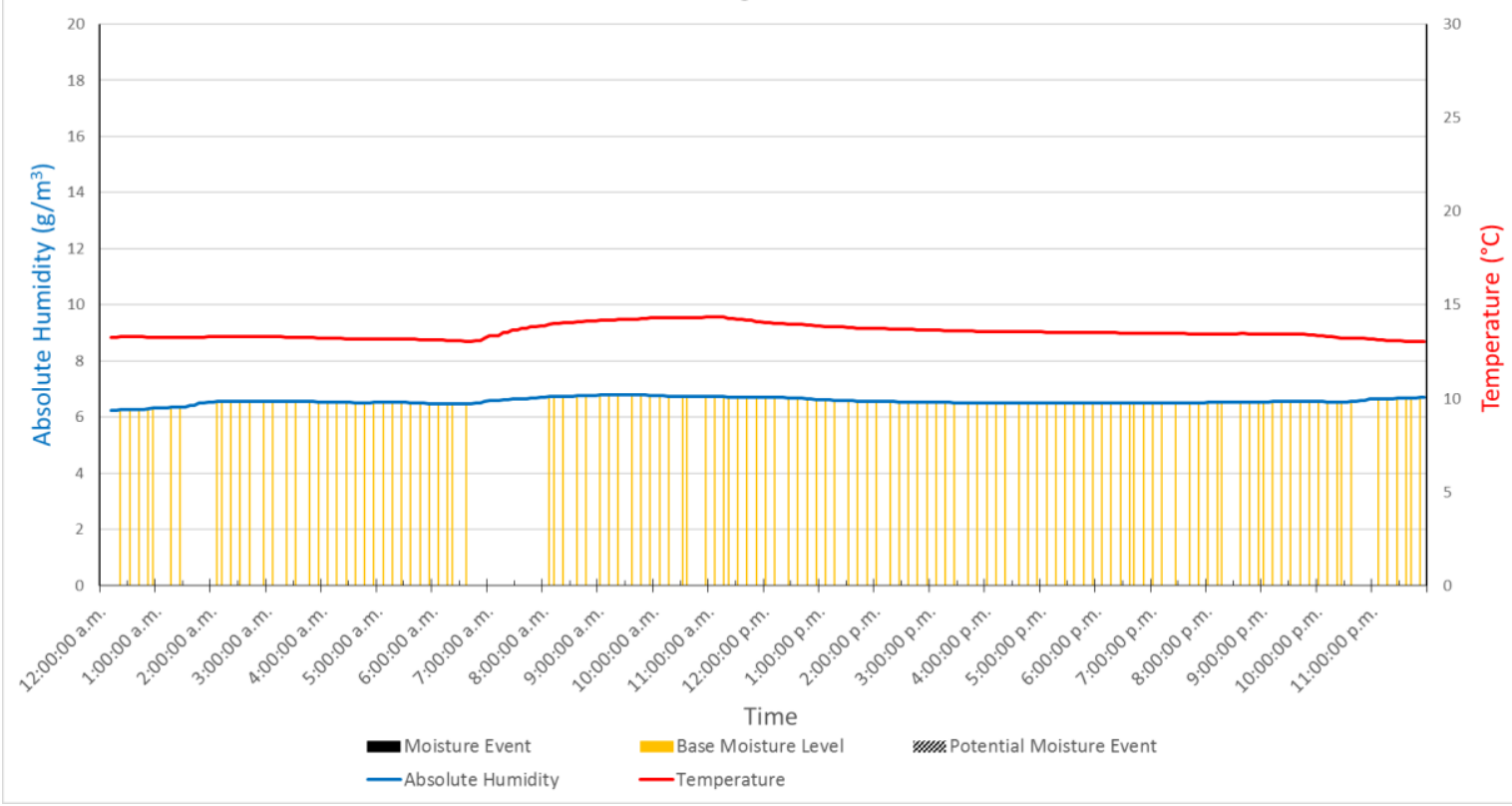

Figure 61 Typical Day: No Change

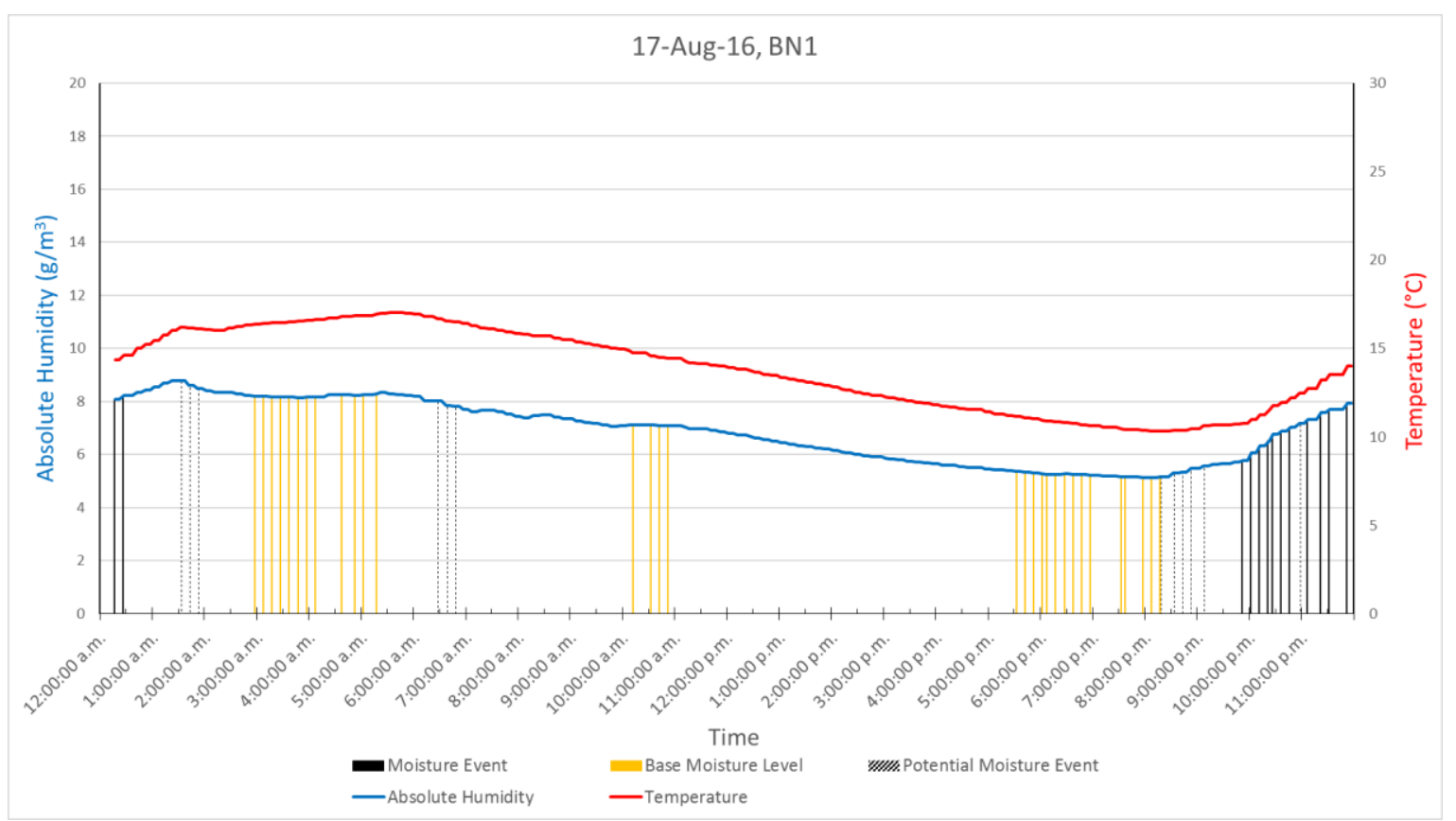

Figure 62 Typical Day: Night Increase 
03-Aug-16, BN1

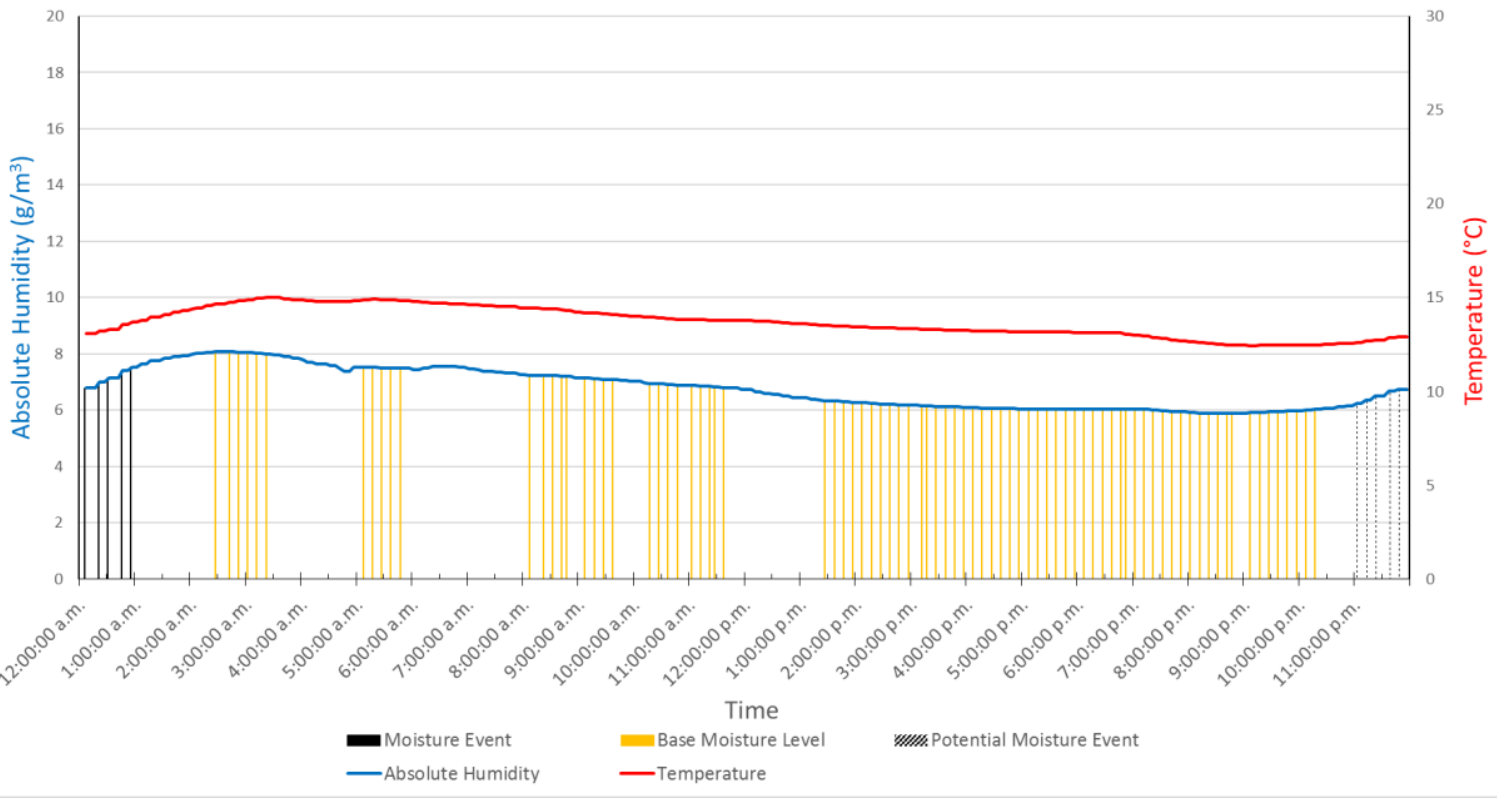

Figure 63 Typical Day: Morning Increase

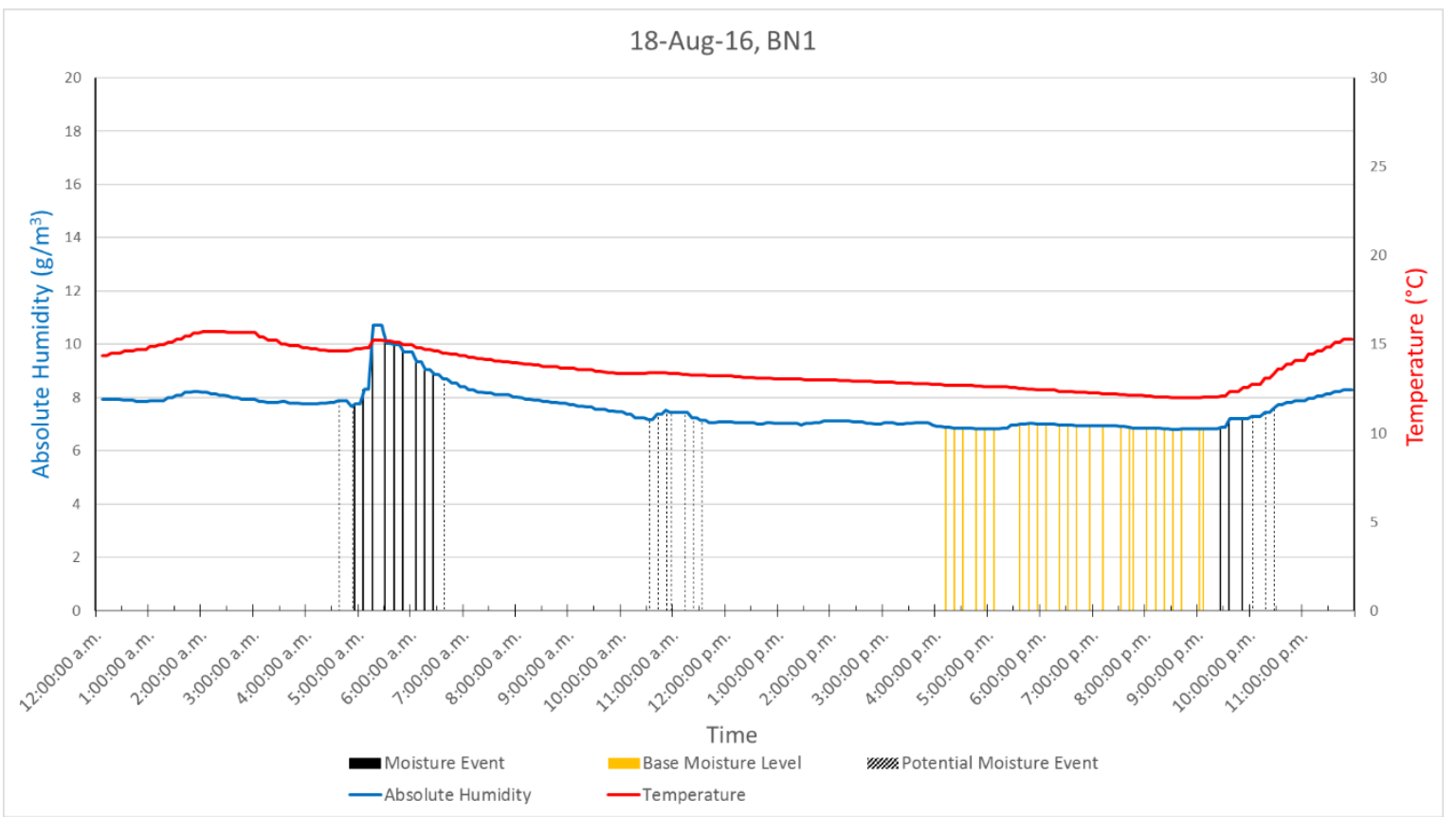

Figure 64 Typical Day: Episode

The moisture events classified as episodes in House BN1.1 appear to have very little impact on the indoor environment. The absolute humidity would quickly return to a level similar to the starting absolute humidity. The absolute humidity would either reach a base moisture level or otherwise increase independently of any event within a short span of time. Some 
days did not follow this trend, such as 19/08/2016. On this day the event was spread over a period of 2 hours 15 minutes, showing a continuous decrease in absolute humidity and temperature until $8.15 \mathrm{pm}$, over 9 hours after the start of the event.

A moisture event registering as a decrease occurs on only three of the 61 days. All of these occur in the morning. No episode took place directly before any decrease. This type of day is too rare to be considered a typical day.

\subsection{Example of Producing Typical Days: BN1.2}

There were 12 days involving an increase between $8.00 \mathrm{pm}$ and $12.00 \mathrm{pm}$ in the second bathroom of house BN1. While this was occasionally followed by an episode between 12.00am and 4.00am the next day (five out of 12 days), these increases were not commonly associated with an episode.

Only two days had no moisture event take place. The most common type of moisture event was an episode, with 132 episodes taking place over a period of 61 days. Only three days had no episode take place. The most common time for episodes to take place was during the morning from 8.00am - 12.00pm (34/61) and 4.00am - 8.00am (33/61). Episodes were also common at night, commonly occurring from 4.00pm - 8.00pm (29/61) and 8.00pm 12.00am (26/61).

Eighteen days had an episode both from 4.00pm - 8.00pm and 8.00pm - 12.00am. Days which had two episodes at night were unlikely to also have two episodes during the morning $(3 / 18)$ or to have no episode take place in the morning (4/18).

Fourteen days had an episode both from 4.00am - 8.00am and 8.00am - 12.00pm. 4/14 days had no episode take place that night. $6 / 14$ days had one episode take place that night. 4/14 days had two episodes take place that night.

The most common type of days was one morning episode, two night episodes (11/61). There were a total of nine different types of days with minor variations between them:

- no moisture events;

- no morning episodes, one night episode;

- no morning episodes, two night episodes; 
- one morning episode, no night episodes;

- one morning episode, one night episode;

- $\quad$ one morning episode, two night episodes;

- two morning episodes, no night episodes;

- two morning episodes, one night episode; and

- two morning episodes, two night episodes.

However, these days shared many similarities and can be further grouped. The typical days were:

- no morning episodes, one or more night episodes;

- one or more morning episode, no night episodes;

- one or more morning episodes, one or more night episodes.

These typical days can then be compared with the episodes. For example, if episodes that take place in the morning are found to influence episodes that take place later that day, then these findings could be shown to be relevant to however many days fall into the third category of typical days (one or more morning episode, one or more night episodes). 
Alister Stubbe

122

Typical Days 


\section{Chapter 9: Summary of Individual Houses}

\subsection{Typical Day Summary}

The description provided of each bathroom in this chapter is based on the typical day definitions developed in 7.5 Filtering Moisture Events for Typical Days. These are based on the moisture event categories defined in 7.4 Defining Categories of Moisture Events. In every bathroom except BN1.1, episodes were the most common type of moisture event. The number of times a moisture event occurred during a specific situation is listed as a fraction of the total number of days. For example, if every day in August and September was measured and episodes took place on ten of these, then this would be listed as (10/61). If eight of these episodes took place from 8.00am - 12.00pm then this would be listed as $(8 / 61)$ when referencing this specific period.

\subsection{Mean and Median Absolute Humidity Results}

The mean and median absolute humidity at the start, peak, and end of each episode are contained in a graph alongside the typical day summary for each house. For the purposes of calculating the mean and median absolute humidity, episodes were counted regardless of context (such as potential moisture events, length, and change in absolute humidity). Using both the mean and median mitigated this issue, allowing situations where outliers distorted the results to be identified. 


\subsection{Results Summary BN1.1}

In the first bathroom of house BN1, many days had no moisture events take place (17/61).

The most common type of moisture event was an increase, with 43 increases taking place over a period of 61 days. This was the only bathroom where an episode was not the most common type of moisture event. The most common time for an increase to occur was from 8.00pm - 12.00am (35/61) followed by 12.00am - 4.00am (7/61). Episodes (Figure 65) were uncommon (9/61) and occurred throughout the day. Typical days were:

- no moisture event;

- $\quad$ night increase;

- morning increase; and

- episode.

\subsubsection{Example of Interpreting Graph}

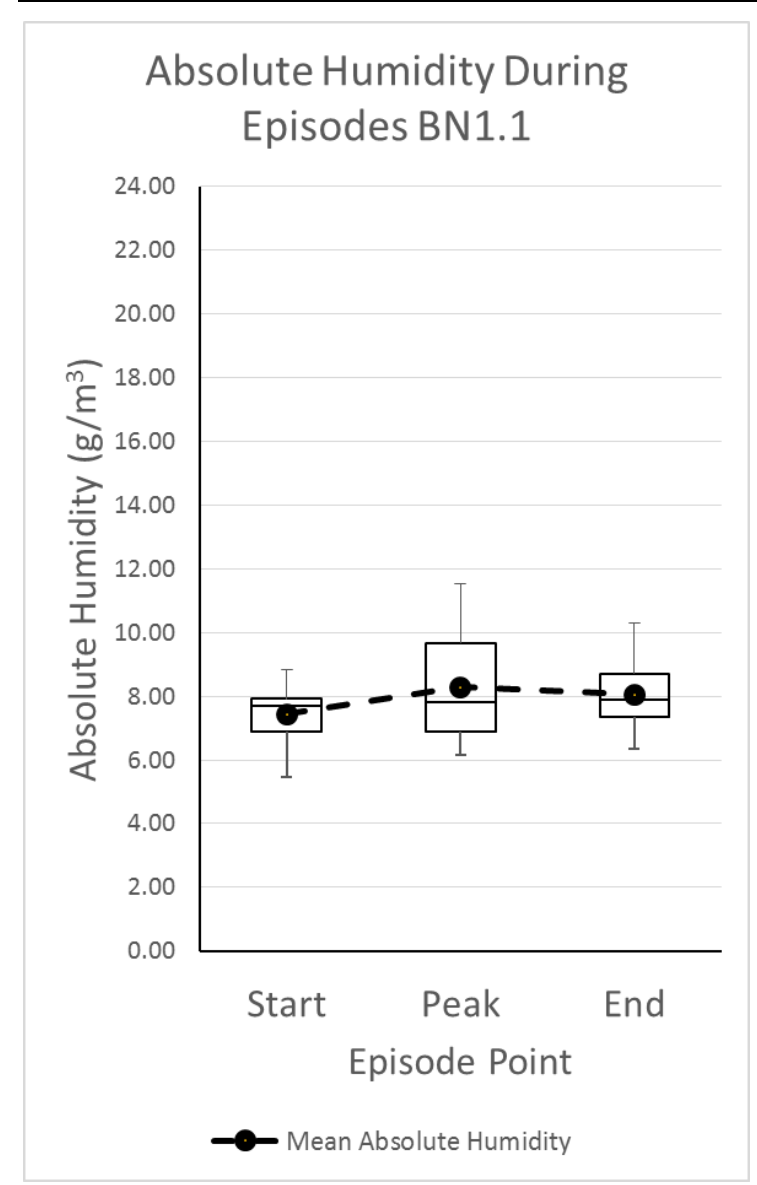

Figure 65 Average Absolute Humidity for Episodes in Bathroom BN1.1
Figure 65 shows the first of two bathrooms (BN1.1) in house BN1. This type of graph is designed to be compared to the typical day information. The spacing between the start, peak, and end does not represent the amount of time elapsed between them. The start of the episode is reasonably consistent, with half of the results taking place within a range of $1 \mathrm{~g} / \mathrm{m}^{3}$. A quarter of episodes began at just under $8 \mathrm{~g} / \mathrm{m}^{3}$. The mean absolute humidity was slightly lower than the median.

Once the episode reached its peak, the results became more spread out. Half of the results took place within a range of $3 \mathrm{~g} / \mathrm{m}^{3}$. Interestingly, the median remained at a similar level, although the minimum increased to above $6 \mathrm{~g} / \mathrm{m}^{3}$. The mean absolute humidity 
increased to above the median.

The results became less spread out by the end of the episode, with half the results taking place within a range less than $2 \mathrm{~g} / \mathrm{m}^{3}$. The median continued to remain at a similar level while the mean decreased, ending just above the median.

\subsubsection{Example of Comparison to Typical Days}

The most common type of moisture event experienced in house BN1.1 was an increase. Only 7/61 days experienced an episode large enough to qualify for the typical day analysis. This means that Figure 65 represented only a small number of the moisture events which take place. It may potentially mean that some of the 'episodes' being counted are increases that happened to end with a small decrease. This would explain the mean absolute humidity at the end of the episodes being similar to the mean absolute humidity at the peak. The main difference between them is the range of results, with the measurements ending within a small absolute humidity range than the peak. 


\subsection{Results Summary BN1.2}

Unlike BN1.1, few days had no moisture event take place in the second bathroom of house

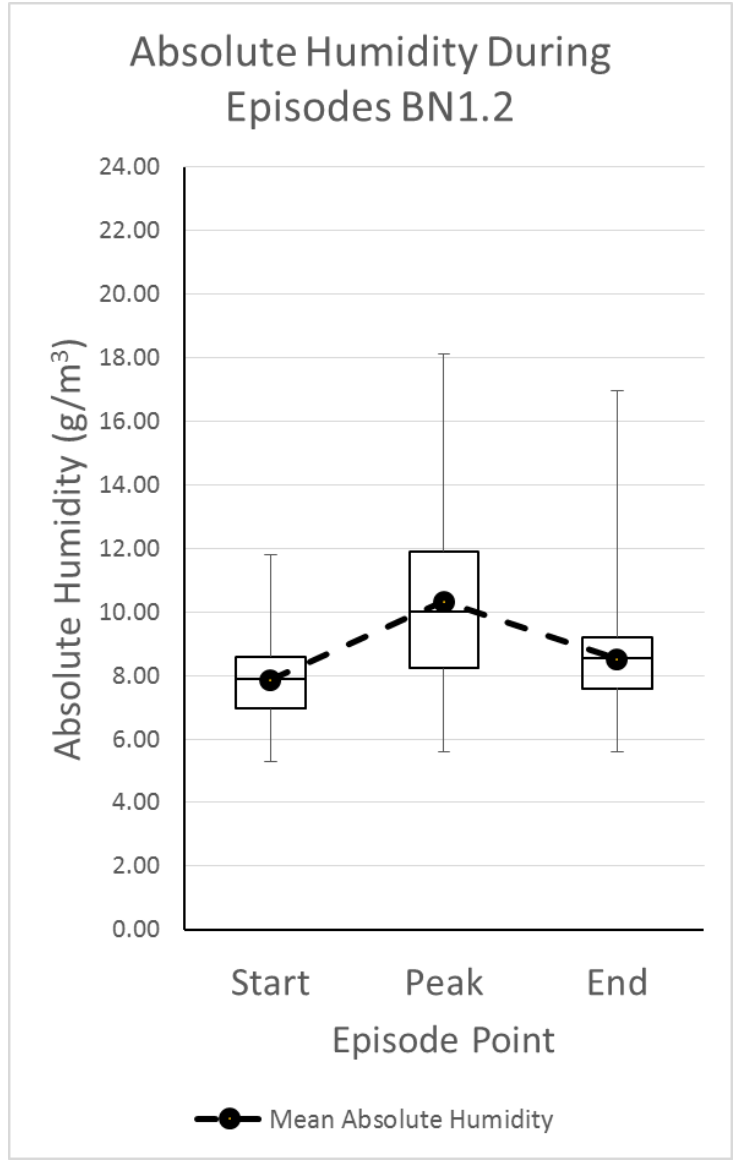

Figure 66 Average Absolute Humidity for Episodes in Bathroom BN1.2
BN1.2 (2/61). Episodes (Figure 66) were the most common type of moisture event with 132 episodes taking place over a period of 61 days. Episodes were common during many periods of the day including 4.00am - 8.00am (33/61), 8.00am - 12.00pm (34/61), 4.00pm 8.00pm (29/61), and 8.00pm - 12.00am (26/61). It was common for three or more episodes to take place on a single day (26/61). Typical days were:

- no morning episodes, one or more night episodes;

- one or more morning episode, no night episodes;

- one or more morning episodes, one or more night episodes. 


\subsection{Results Summary BN2}

Few days had no moisture event take place in the bathroom of house DN2 (4/61). Episodes (Figure 67) were the most common type of moisture event with 143 episodes taking place over a period of 61 days. These typically occurred from either 4.00am -8.00am (42/61) or $8.00 \mathrm{pm}-12.00 \mathrm{am}$ (44/61). It was also common for episodes to occur from either 8.00am -

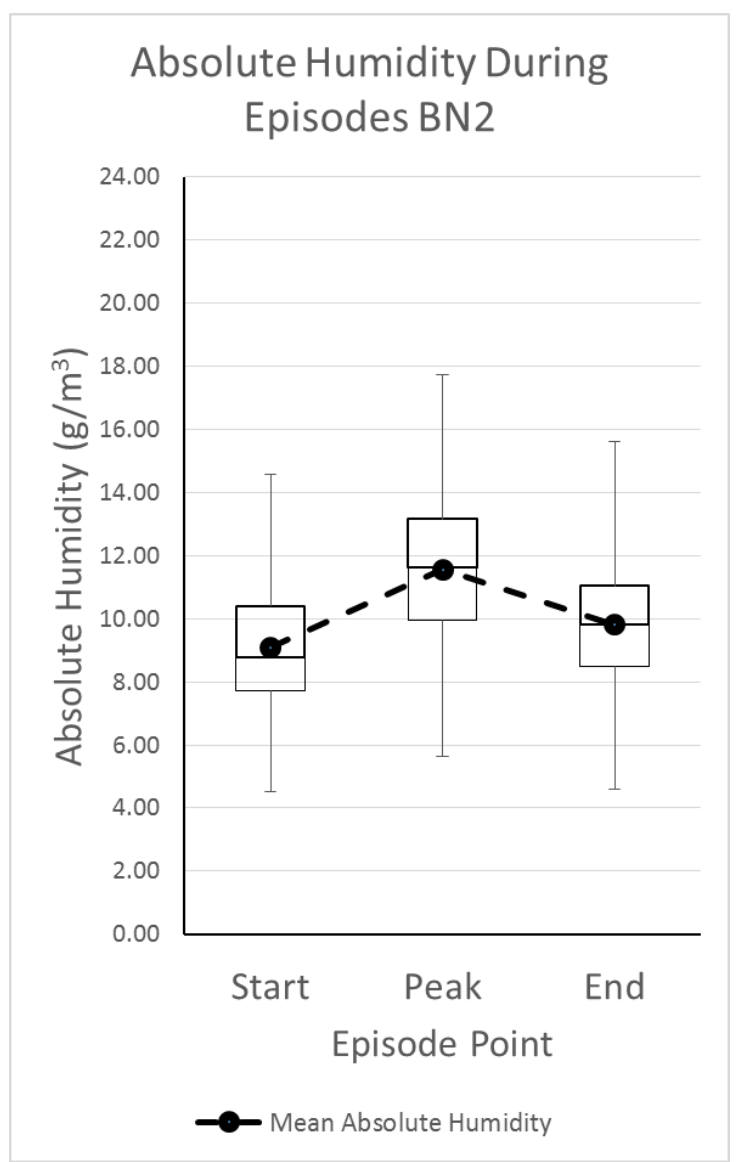

Figure 67 Average Absolute Humidity for Episodes in the Bathroom of House BN2
$12.00 \mathrm{pm}(24 / 61)$ or $12.00 \mathrm{pm}-4.00 \mathrm{pm}$

(22/61). Only six days with an event from

4.00am to 8.00am did not have an event take place that same day from 8.00pm - 12.00am.

Typical days were:

- no moisture event;

- one or more morning episodes, one or more night episodes, nothing in between;

- one or more morning episodes, one or more night episodes, one episode in between;

- one or more morning episodes, one or more night episodes, two episodes in between; and

- one early morning episode. 


\subsection{Results Summary DN1}

Every day had at least one moisture event take place in the bathroom of house DN1. The most common type of moisture event was an episode (Figure 68), with at least one episode taking place every day. 109 episodes took place over a period of 61 days. The most common time for an episode to occur was from 8.00pm - 12.00am (41/61) followed by 4.00am $8.00 \mathrm{am}$ (30/61). 18/61 days had an episode take place during both of these periods. Of the

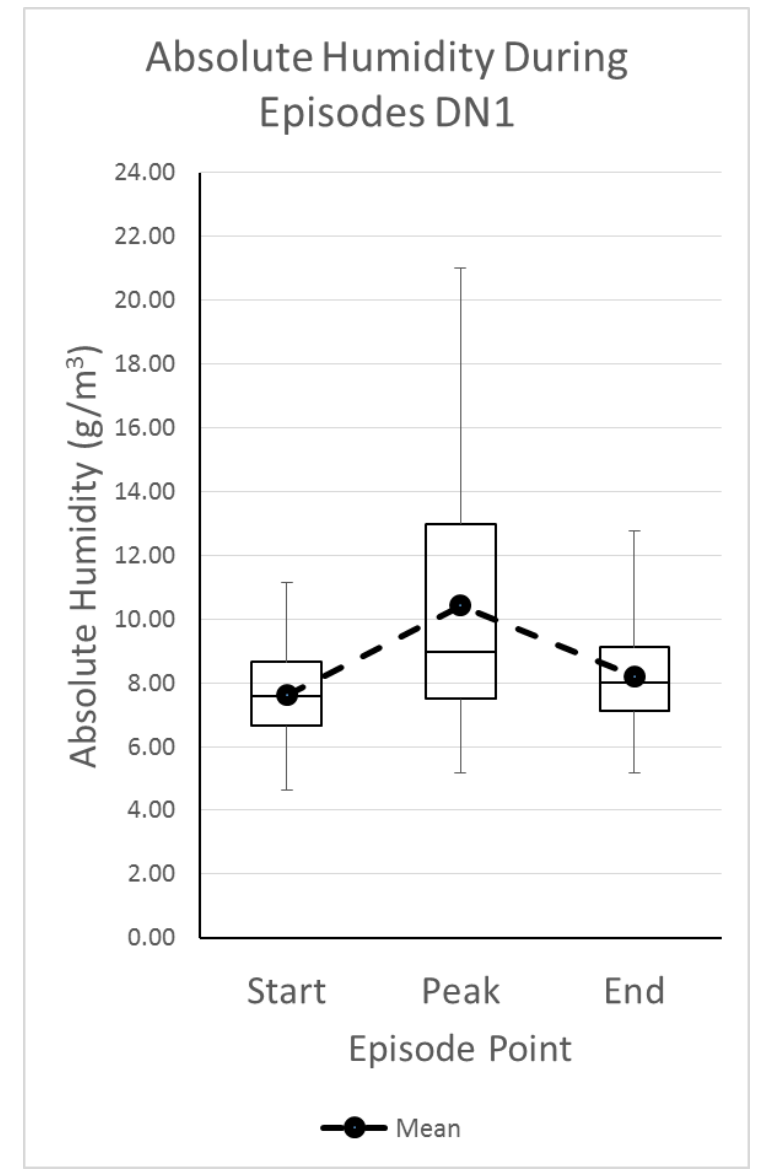

Figure 68 Average Absolute Humidity for Episodes in the Bathroom of House DN1
20 days where an episode did not take place from 8.00pm - 12.00am, 19 had an episode take place from $4.00 \mathrm{pm}-8.00 \mathrm{pm}$. No days contained an episode during both of these periods. Only one episode took place from 12.00pm-4.00pm. 24 days contained two episodes and no other moisture event. Ten days had three episodes and no other moisture events. Three days contained an increase. Four days contained a decrease. Typical days were:

- two morning episodes, one evening episode;

- one morning episode, one evening episode; and

- no morning episodes, 1 evening episode. 


\subsection{Results Summary DN2}

Every day had at least one moisture event take place in the bathroom of house DN2. The

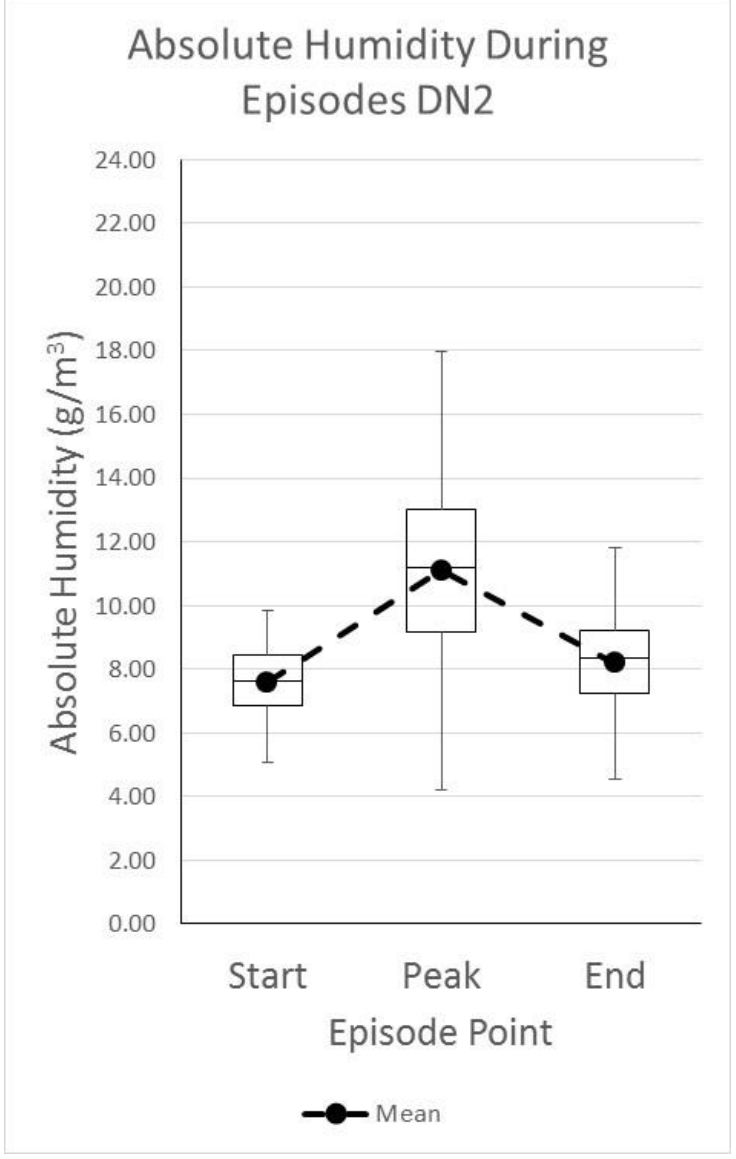

Figure 69 Average Absolute Humidity for Episodes in the Bathroom of House DN2 most common type of moisture event was an episode (Figure 69), with 166 episodes taking place over a period of 61 days. The most common times for an episode to occur was from 4.00am - 8.00am (53/61) and 8.00pm 12.00am (46/61). Every day had an episode take place during one of these two periods. Every day that did not have an episode take place from 8.00pm - 12.00am had an episode take place from $4.00 \mathrm{pm}-8.00 \mathrm{pm}$. Only one episode took place from $12.00 \mathrm{pm}-4.00 \mathrm{pm}$. Typical days were:

- two morning episodes, one evening episode;

- one morning episode, one evening episode; and

- two evening episodes. 


\subsection{Results Summary DN4}

Only one day had no moisture event take place in the bathroom of house DN4 (1/61). The most common type of moisture event was an episode (Figure 70), with 128 episodes taking

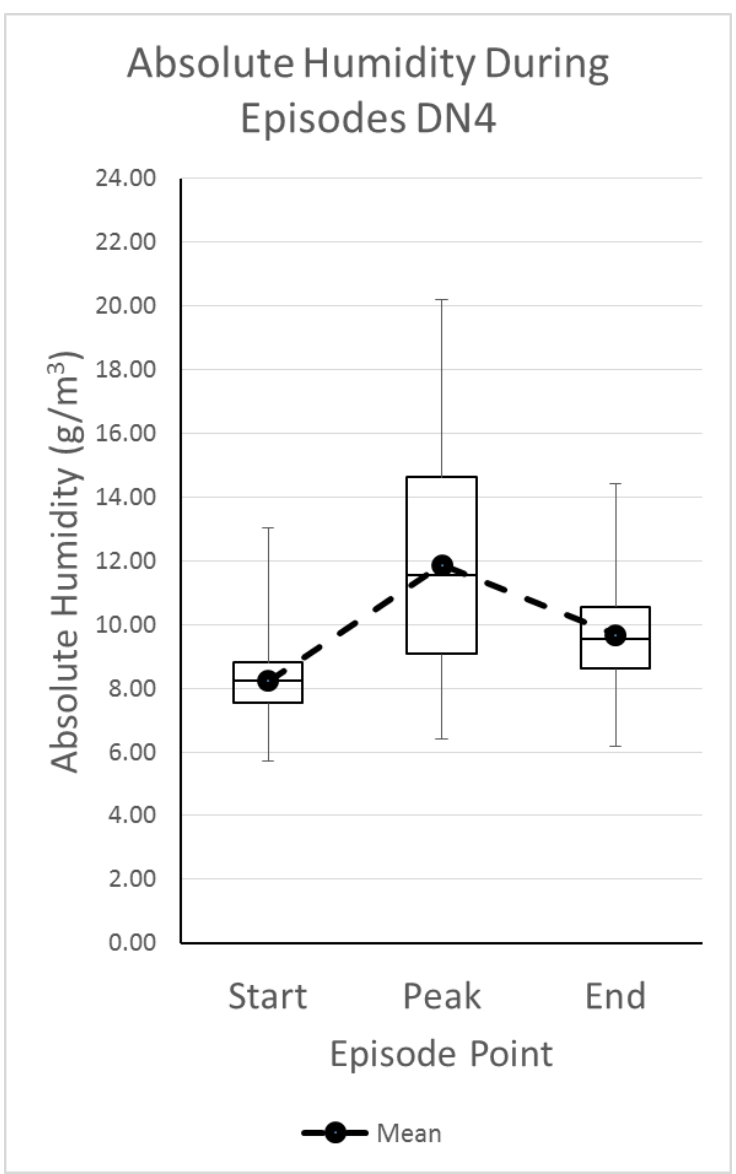

Figure 70 Average Absolute Humidity for Episodes in the Bathroom of House DN4 place over a period of 61 days. The most common time for an episode to occur was from 8.00am - 12.00pm (59/61). Episodes commonly took place from either $4.00 \mathrm{pm}-$ 8.00pm (32/61) or 8.00pm - 12.00am (28/61) as well. The majority of days had an episode take place during at least one of these periods (51/61). No episode took place from $12.00 p m$ -4.00pm. Typical days were:

- one or more morning episodes, 4.00pm $-8.00 \mathrm{pm}$ episode;

- one or more morning episodes, 8.00pm $-12.00 a m$ episode;

- one or more morning episodes, two evening episodes; and

- one or more morning episodes, no evening episode. 


\subsection{Results Summary DN6.1}

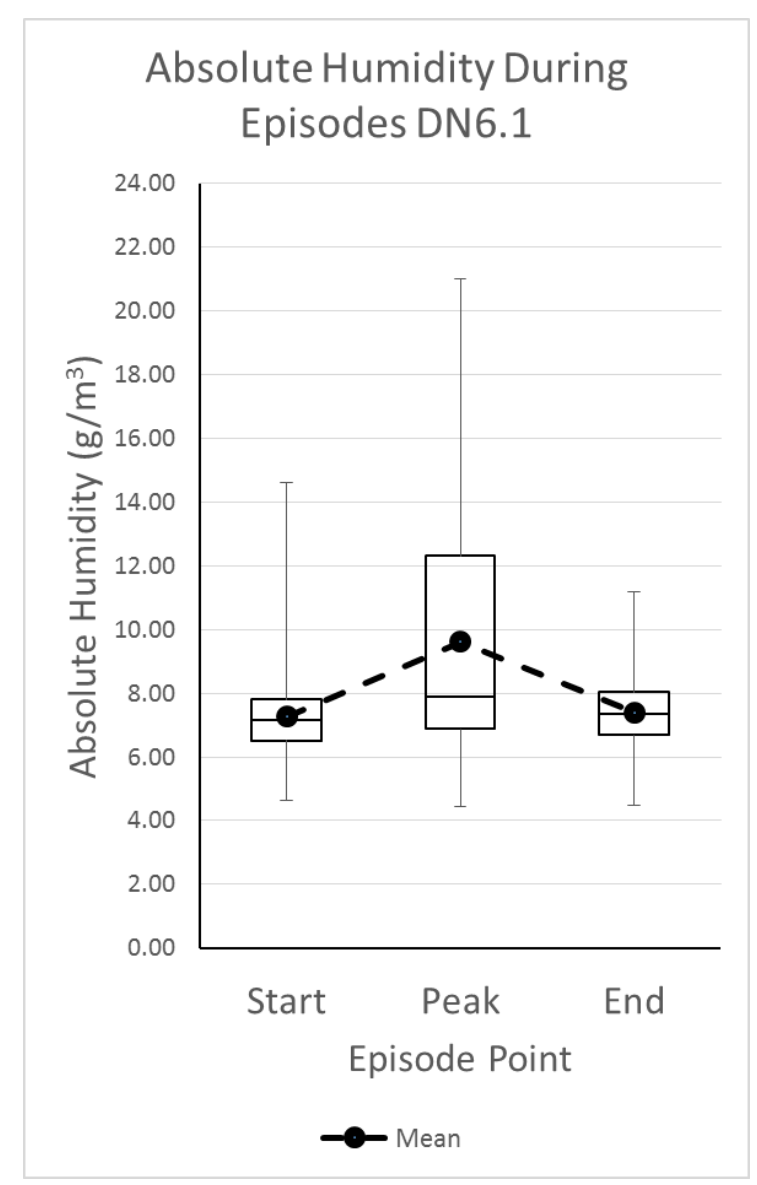

Figure 71 Average Absolute Humidity for Episodes in Bathroom DN6.1
A large number of days had no moisture events take place in the first bathroom of house DN6 (28/61). The most common type of moisture event was an episode (Figure 71), with 28 episodes taking place over a period of 61 days. The common time for an episode to occur was from 8.00pm - 12.00am (20/61).

Although episodes would take place infrequently, when they did take place they reached high levels of absolute humidity and lasted for several hours. The absolute humidity would increase over an hour before rapidly decreasing. Typical days were:

- no moisture event;

- one or more evening episodes; and

- one or more morning episodes. 


\subsection{Results Summary DN6.2}

Like DN6.1, a large number of days had no moisture events take place in the second

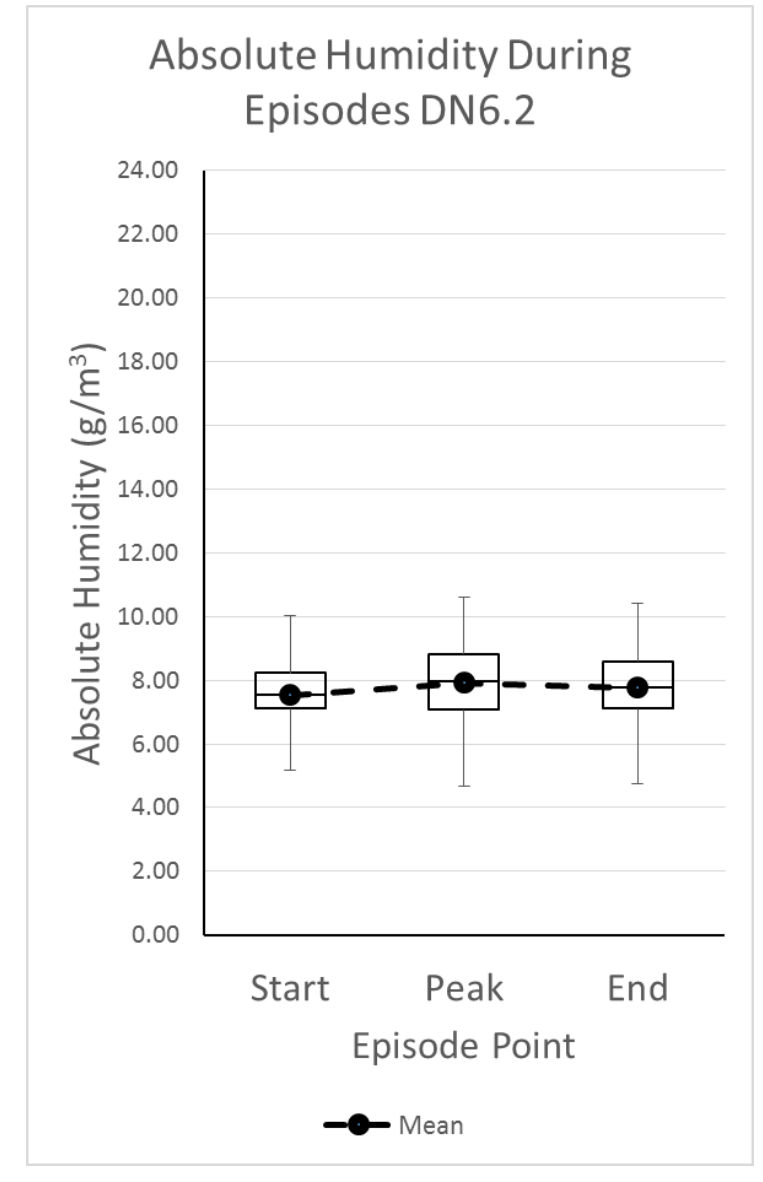

Figure 72 Average Absolute Humidity for Episodes in Bathroom DN6.2 bathroom of house DN6 (29/61). On many of these days there were also no moisture events in the first bathroom, DN6.1 (22/61). The most common type of moisture event was an episode (Figure 72), with 20 episodes taking place over a period of 61 days. The most common time for an episode to occur was from 8.00pm - 12.00am (14/61). Episodes would occasionally take place from 12.00am - 4.00am (4/61). Increases were also common (13/61) most commonly taking place from 4.00am - 8.00am (6/61). Typical days were:

- no moisture event;

- one or more morning episodes; and

- one or more night episodes. 


\subsection{Results Summary LH2}

Few days had no moisture events take place in the bathroom of house LH2 (2/61). The most common type of moisture event was an episode (Figure 73), with 130 episodes taking place over a period of 61 days. These typically occurred from either $4.00 \mathrm{am}-8.00 \mathrm{am}$ (56/61) or

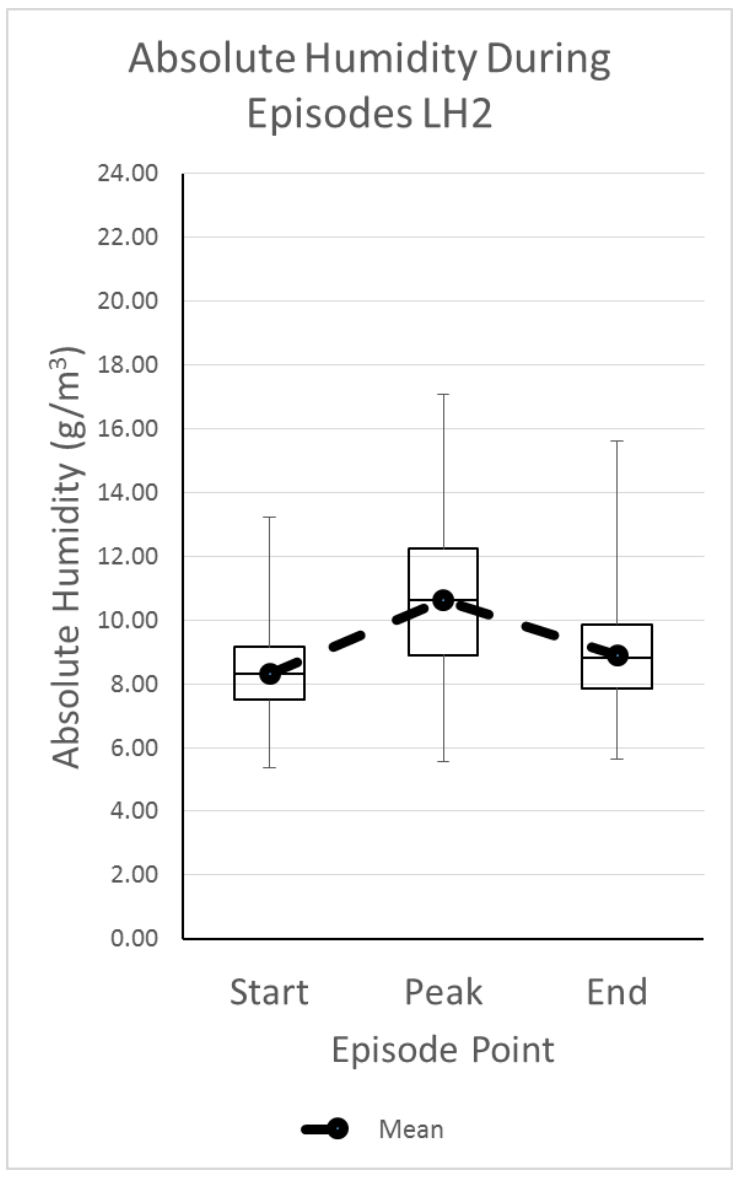

Figure 73 Average Absolute Humidity for Episodes in the Bathroom of House LH2 8.00pm - 12.00am (52/61). This is an

overwhelming majority of the days, showing a consistent pattern. Only two days had no moisture change. Some days had an episode between 8.00am - 12.00pm (13/61) or $4.00 \mathrm{pm}-8.00 \mathrm{pm}(7 / 61)$. There were no cases of episodes occurring during both these periods on the same day. Typical days were:

- No moisture event

- one or more morning episodes, one or more night episodes, nothing in between

- one or more morning episodes, one or more night episodes, one episode from $8.00 \mathrm{am}-12.00 \mathrm{pm}$

- one or more morning episodes, one or more night episodes, one episode from $4.00 p m-8.00 p m$ 


\subsection{Results Summary MT1}

Only one day had no moisture event take place in the bathroom of house MT1 (1/61). The

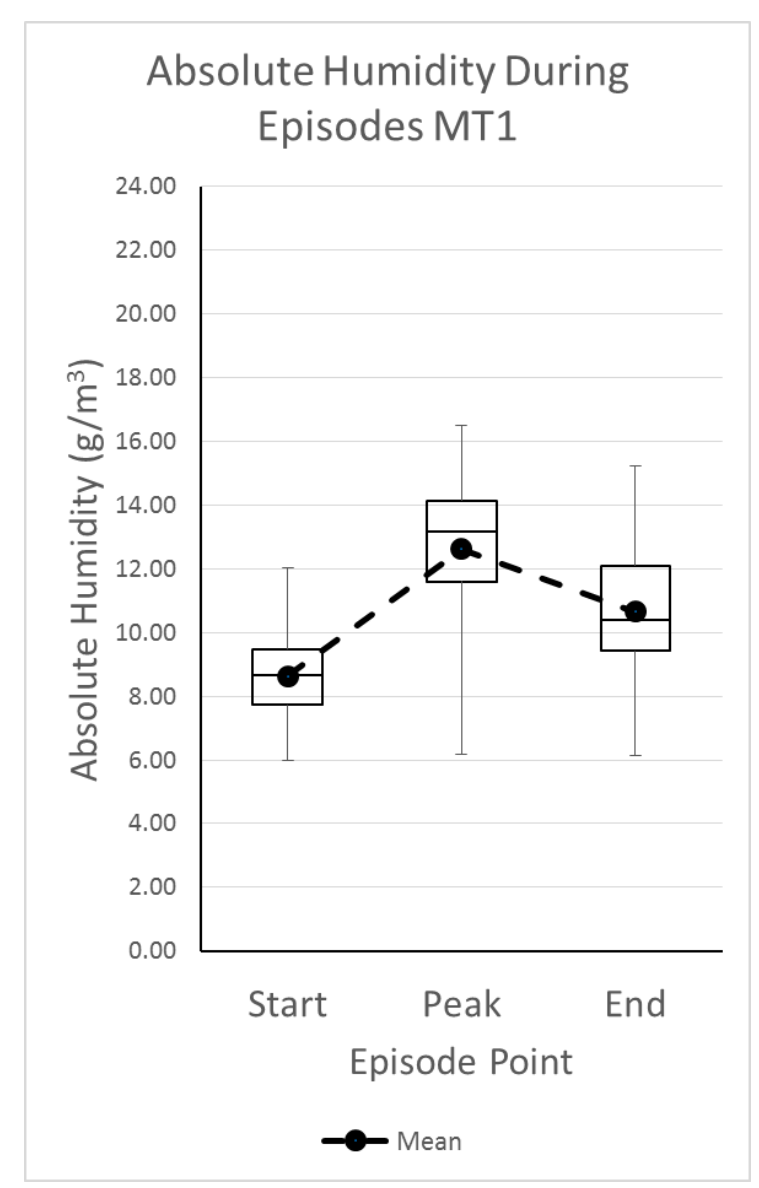

Figure 74 Average Absolute Humidity for Episodes in the Bathroom of House MT1 most common type of moisture event was an episode (Figure 74), with 60 episodes taking place over a period of 61 days. Episodes typically occurred from 8.00am - 12.00pm (39/61), although they would occasionally occur slightly later, taking place from $12.00 \mathrm{pm}$ $-4.00 p m(11 / 61) .8 / 11$ of these later episodes did not have an episode take place from 8.00am - 12.00pm that same day. Increases typically took place late at night, from 8.00pm -12.00am (21/61). Typical days were:

- no moisture event;

- one or more episodes and no increase;

- one or more episodes and an increase; and

- no episodes and an increase. 


\subsection{Results Summary MT2.1}

Few days had no moisture event take place in the bathroom of house MT2.1 (6/61). The most common type of moisture event was an episode (Figure 75), with 103 episodes taking

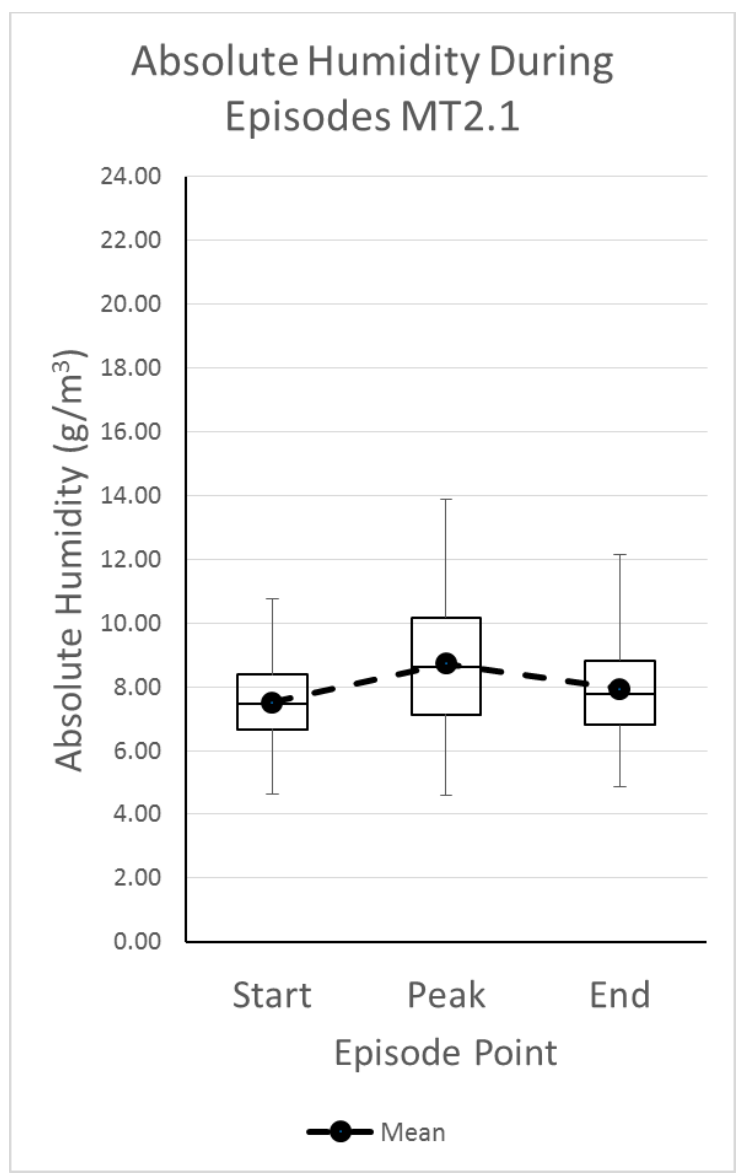

Figure 75 Average Absolute Humidity for Episodes in Bathroom MT2.1 place over a period of 61 days. Throughout the morning, episodes became more common closer to midday. $15 / 61$ days had an episode take place from 12.00am - 4.00am, 25/61 days from 4.00am - 8.00am, and 44/61 from 8.00am - 12.00pm. 14/61 days had an episode take place from 8.00pm - 12.00am. 12 of these days also had an episode take place from 8.00am - 12.00pm. Typical days were:

- no moisture event;

- one episode from 8.00am - 12.00pm, no other episode;

- one episode from 8.00am - 12.00pm, one or more morning episodes; and

- 8.00am - 12.00pm episode, one or more night episodes. 


\subsection{Results Summary MT2.2}

Unlike MT2.1, days with no moisture events were common in the bathroom of house MT2.2

$(26 / 61)$. The most common type of moisture event was an episode (Figure 76), with a total

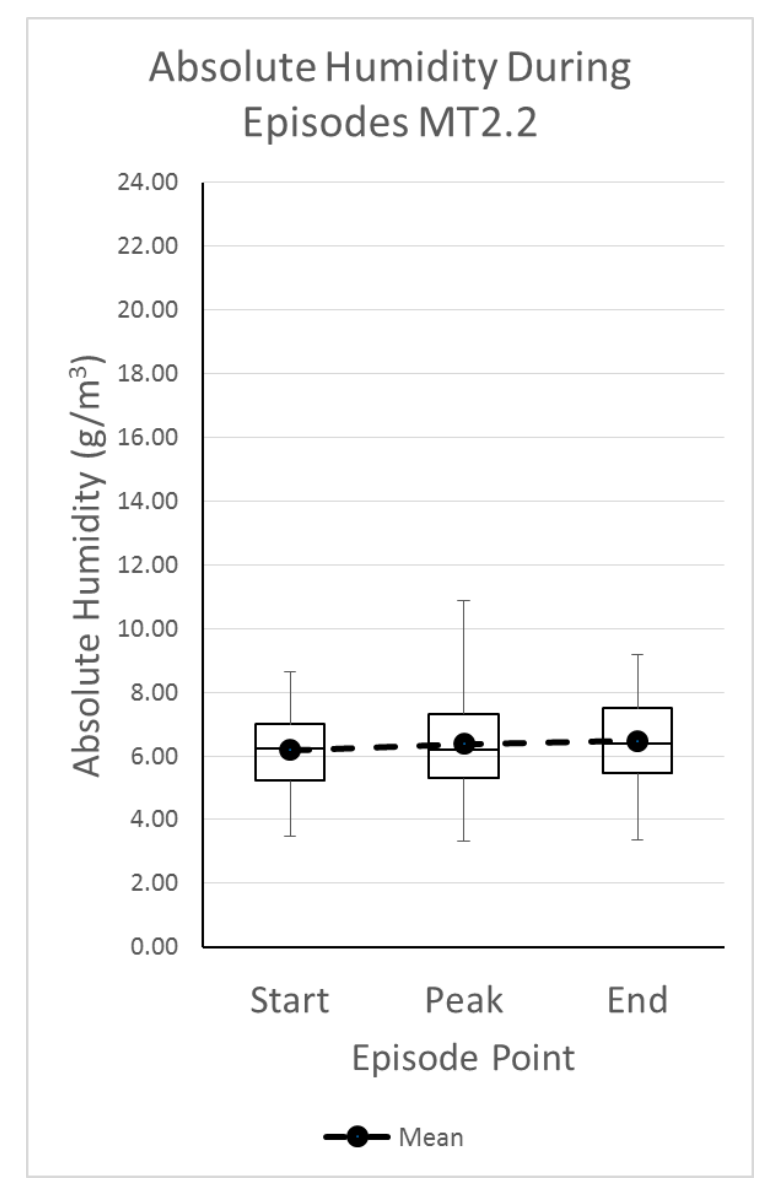

Figure 76 Average Absolute Humidity for Episodes in Bathroom MT2.2 of 32 episodes taking place over a period of 61 days. A total of 21/61 days contained an episode. There was little consistency in when episodes occurred. The most common times were from 12.00am - 4.00am (9/61), 8.00am - 12.00pm (12/61), and 8.00pm - 12.00am (6/61). Out of the days where at least one episode occurred, 13/21 had one episode take place, 5/21 had two episodes take place, and $3 / 21$ had three episodes take place. For all days with three episodes, at least two episodes took place in consecutive periods of time. Typical days were:

- no moisture event;

- one episode;

- two episodes; and

- three episodes. 


\subsection{Results Summary NL1}

Days with no moisture events were common in the bathroom of house NL1, occurring 20/61 times. Notably, 19 of these days took place in a continuous series (1/08/2016 19/08/2016). The most common type of moisture event was an episode (Figure 77), with 67

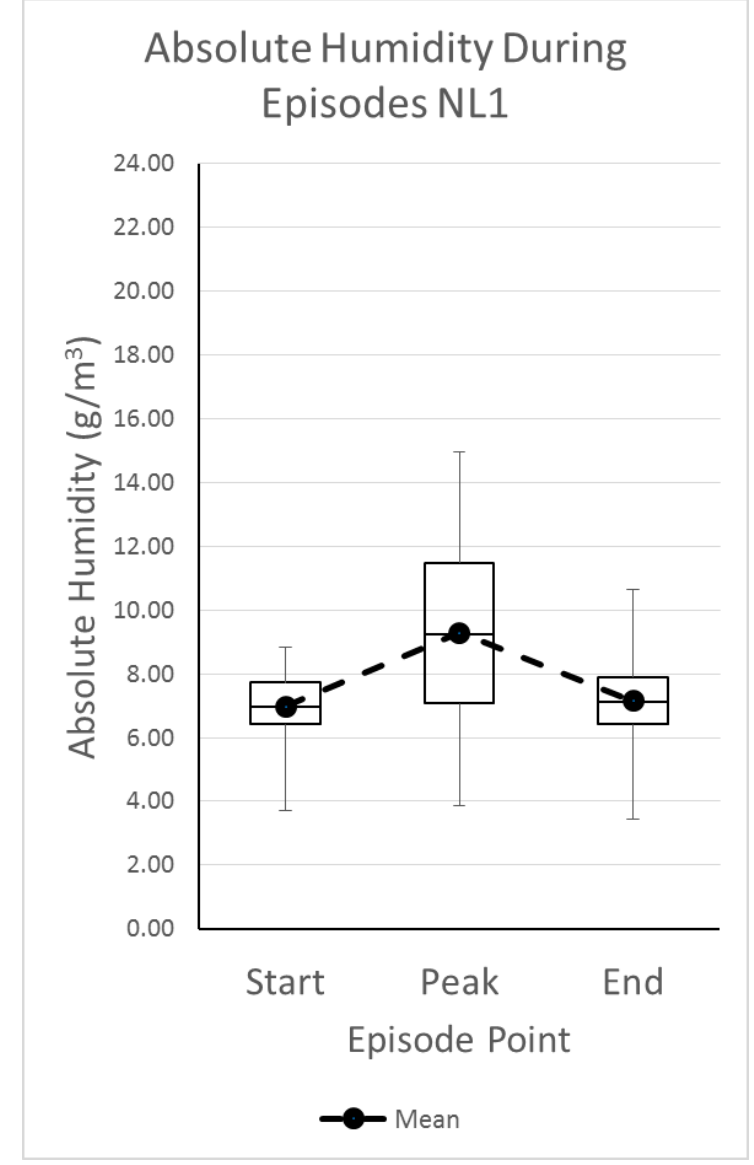

Figure 77 Average Absolute Humidity for Episodes in the Bathroom of House NL1 episodes taking place over a period of 61 days. Episodes most commonly occurred at night from 4.00pm - 8.00pm (20/61) and from 8.00pm - 12.00am (26/61). Only four days had an episode during both of these periods. Episodes also took place in the morning from 4.00am - 8.00am (10/61) and 8.00am $12.00 \mathrm{pm}$ (8/61). No days had an episode take place during both of these periods. All days with an episode in either of these periods also had an episode take place from $4.00 \mathrm{pm}-$ $8.00 \mathrm{pm}$ or $8.00 \mathrm{pm}-12.00 \mathrm{am}$. Typical days were:

- no moisture event;

- one or more night episodes and no morning episode; and

- one or more night episodes and one or more morning episodes. 


\subsection{Results Summary NL2}

Few days had no moisture events take place in the bathroom of house NL2 (5/61). The most common type of moisture event was an episode (Figure 78), with 90 taking place over a period of 61 days. The most common time for an episode to occur was from $4.00 \mathrm{pm}-$ $8.00 \mathrm{pm}(42 / 61)$. Episodes also occurred from 8.00pm - 12.00am (13/61). Eight of these days took place on a day where there was no episode from $4.00 \mathrm{pm}-8.00 \mathrm{pm}$, meaning there was

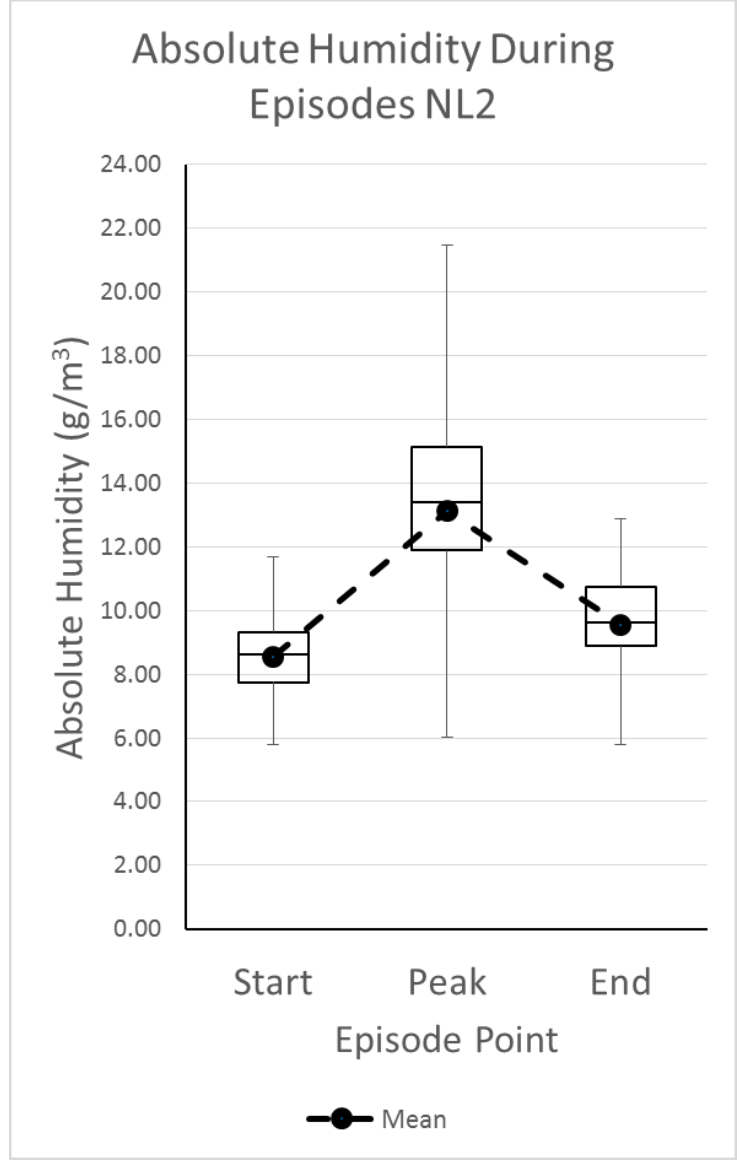

Figure 78 Average Absolute Humidity for Episodes in the Bathroom of House NL2 no clear relationship between these episodes. The second most common time for episodes to occur was from 4.00am - 8.00am (24/61). Only five days which had an episode took place during these times did not also have an episode which took place from 4.00pm $8.00 \mathrm{pm}$. There was only one instance of a day with an episode from 8.00am - 12.00pm (8/61) also having an episode take place from 4.00am-8.00am. Typical days were:

- no moisture event;

- episode from $4.00 \mathrm{pm}-8.00 \mathrm{pm}$, no morning episodes;

- episode from 4.00pm-8.00pm, one or more morning episodes; and

- no 4.00pm-8.00pm episode, one or more morning episodes. 


\subsection{Results Summary WN2}

Absolute Humidity During

Episodes WN2

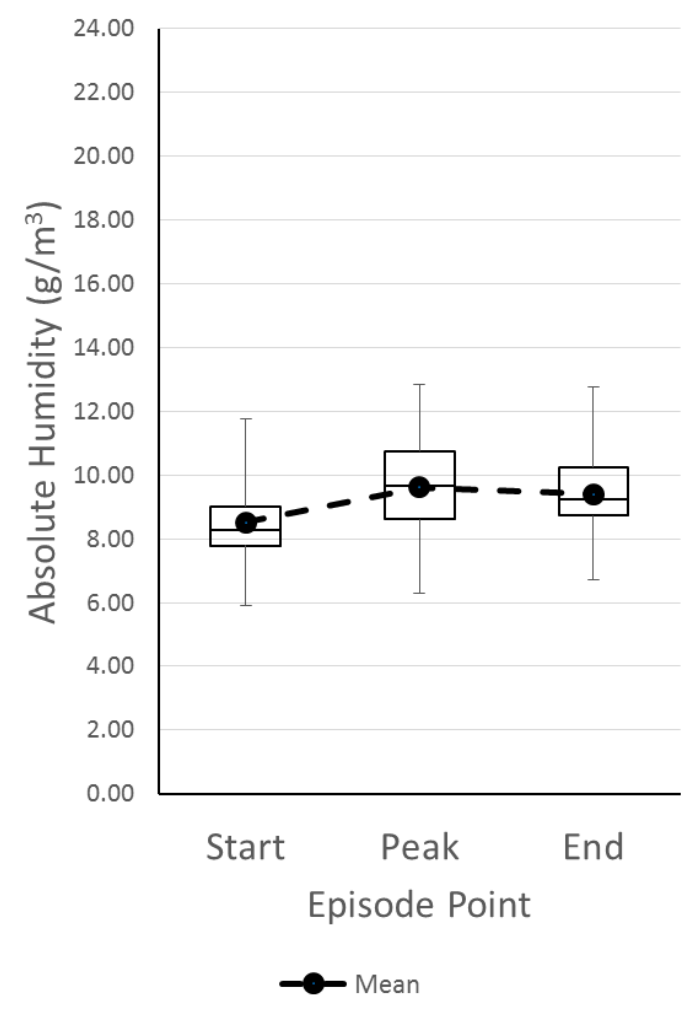

Figure 79 Average Absolute Humidity for Episodes in the Bathroom of House WN2
A large number of days had no moisture events take place in the bathroom of house WN2 (21/55). The most common type of moisture event was an episode (Figure 79), with 34 episodes taking place over a period of 55 days. The most common time for an episode to occur was from $4.00 p m-8.00 p m$ (15/55) followed by 4.00am - 8.00am (12/55). No other period contained more than three episodes over all 61 days. Typical days were:

- no moisture event;

- one morning episode;

- one evening episode; and

- one morning episode and one evening episode. 


\subsection{Results Summary WN3}

There were days where no moisture event took place in the bathroom of house WN3

(10/61). The most common type of moisture event was an episode (Figure 80), with 68 episodes taking place over a period of 61 days. The most common time for episodes to take

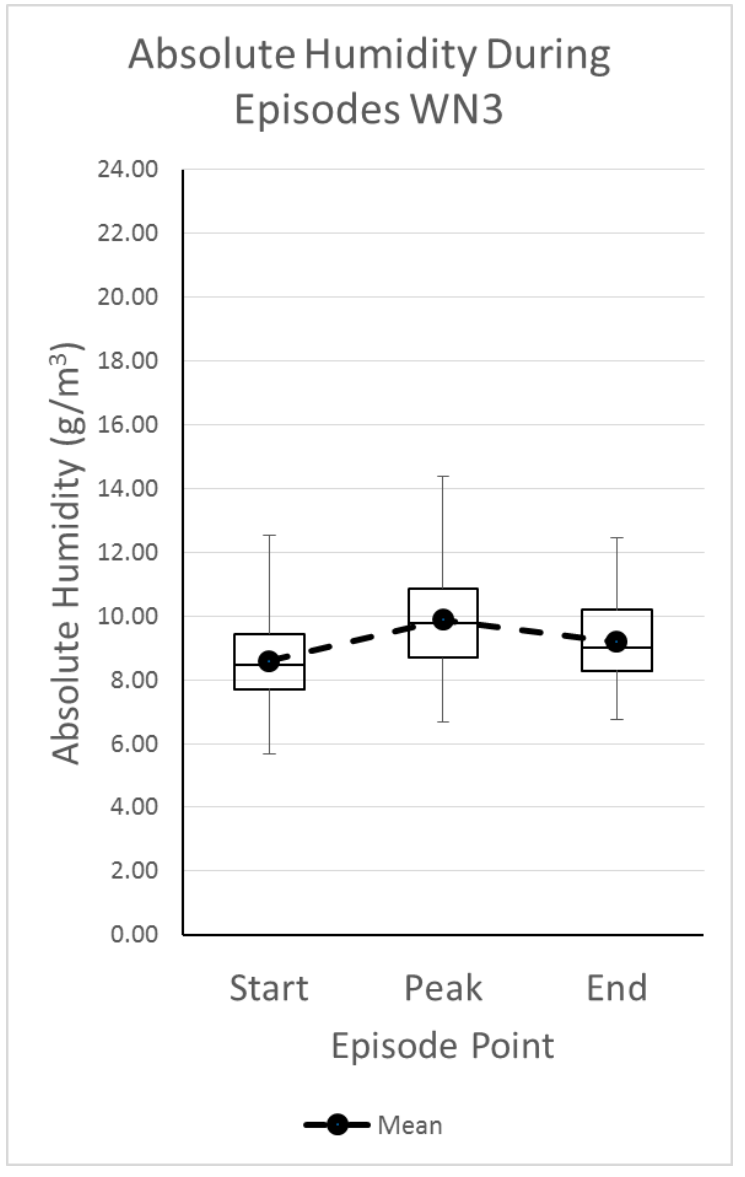
place was from $4.00 \mathrm{pm}-8.00 \mathrm{pm}(34 / 61)$ followed by $8.00 \mathrm{pm}-12.00 \mathrm{am}$ (13/61). No days had an episode take place during both of these periods. Episodes took place throughout the morning, from 12.00am - 4.00am (5/61), 4.00am - 8.00am (9/61), and 8.00am $12.00 \mathrm{pm}$ (7/61). Only one day had an episode take place during more than one of these periods. No episodes took place from 12.00pm - 4.00pm. Typical days were:

- no moisture events;

- one morning episode, one evening episode; and

- no morning episode, one evening episode.

Figure 80 Average Absolute Humidity for Episodes in the Bathroom of House WN3 


\subsection{Results Summary WN4}

Few days had no moisture events take place in the bathroom of house WN4 (5/61). The

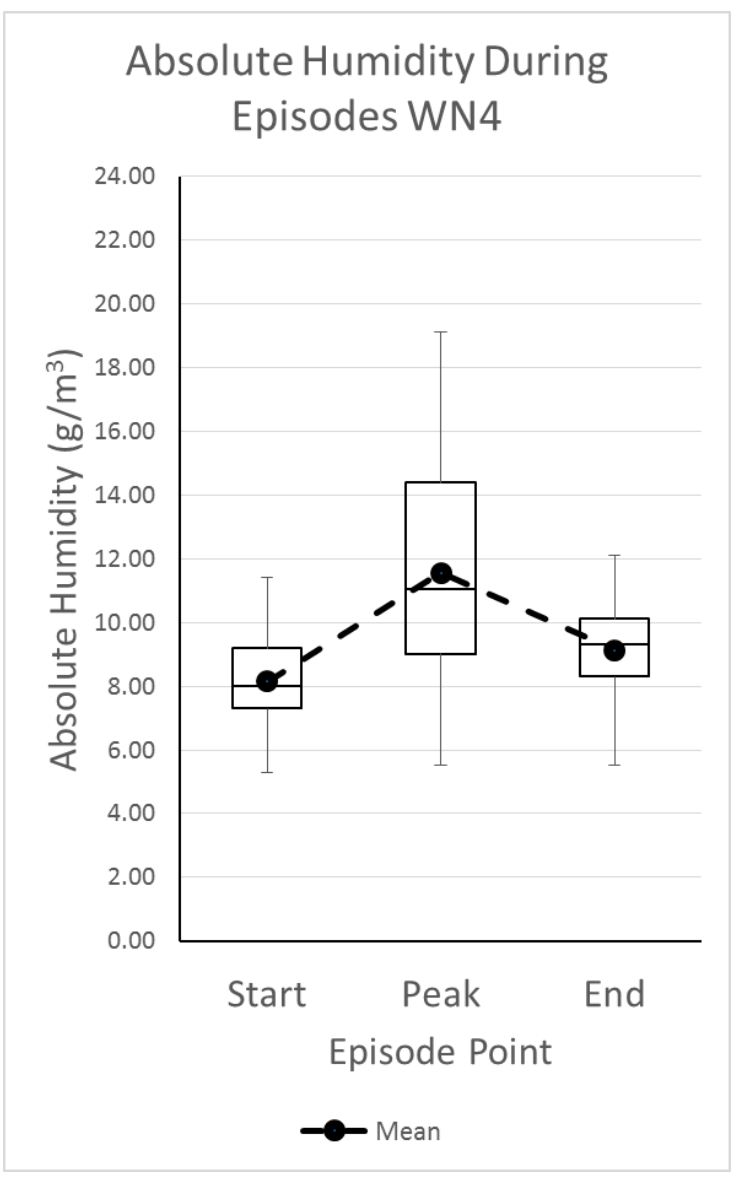

Figure 81 Average Absolute Humidity for Episodes in the Bathroom of House WN4 most common type of moisture event was an episode (Figure 81), with 79 episodes taking place over a period of 61 days. Episodes took place throughout the day. The most common times for episodes to occur were 4.00am 8.00am (22/61), 8.00am - 12.00pm (21/61), and 8.00pm - 12.00an (19/61). There were only four days with an episode both from 4.00am - 8.00am and 8.00am - 12.00pm. Typical days were:

- no moisture event;

- one morning episode, no episode 8.00pm-12.00am;

- one morning episode, one episode $8.00 p m-12.00 a m ;$ and

- no morning episode, one episode $8.00 \mathrm{pm}-12.00 \mathrm{am}$. 


\subsection{Results Summary WN5}

Only one day had no moisture event take place in the bathroom of house WN5 (1/61). The most common type of moisture event was an

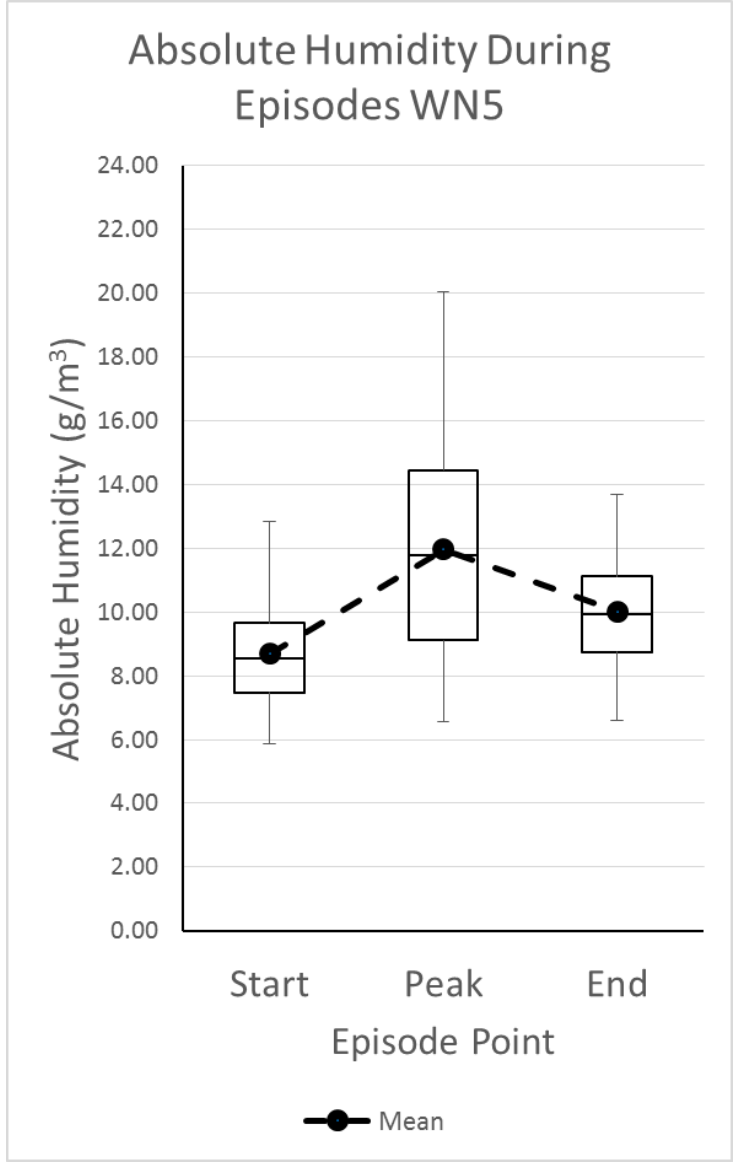

Figure 82 Average Absolute Humidity for Episodes in the Bathroom of House WN5 episode (Figure 82), with 97 episodes taking place over a period of 61 days. The most common time for episodes to take place was from 4.00am - 8.00am (38/61) followed by 8.00am - 12.00pm (19/61). Only one day had an episode take place during both of these periods. Episodes also took place at night from 4.00pm - 8.00pm (15/61) and 8.00pm 12.00am (15/61). No day had an episode take place during both of these periods. Episodes occasionally took place from 12.00am 4.00am (10/61). Typical days were:

- two morning episodes;

- one or more morning episodes, no evening episode; and

- one or more morning episodes, one or more evening episodes. 


\subsection{Results Summary WN6}

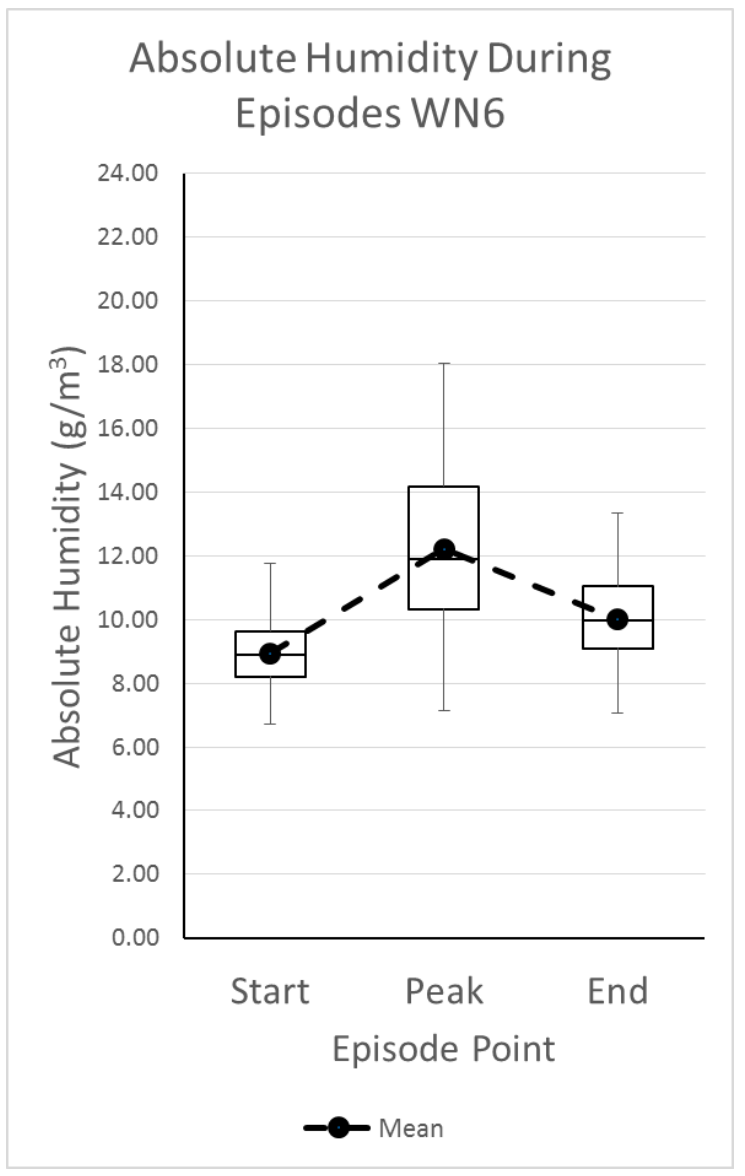

Few days had no moisture events take place

in the bathroom of house WN6 (6/61). The most common type of moisture event was an episode (Figure 83), with 75 episodes taking place over a period of 61 days. The most common time for an episode to take place was from 4.00am - 8.00am (47/61) followed by $8.00 \mathrm{am}-12.00 \mathrm{pm}$ (22/61). It was common for an episode to take place during both of these periods on the same day (18/61).

Typical days were:

- no moisture event;

- one morning episode; and

- two morning episodes.

Figure 83 Average Absolute Humidity for Episodes in the Bathroom of House WN6 


\subsection{Results Summary WN7}

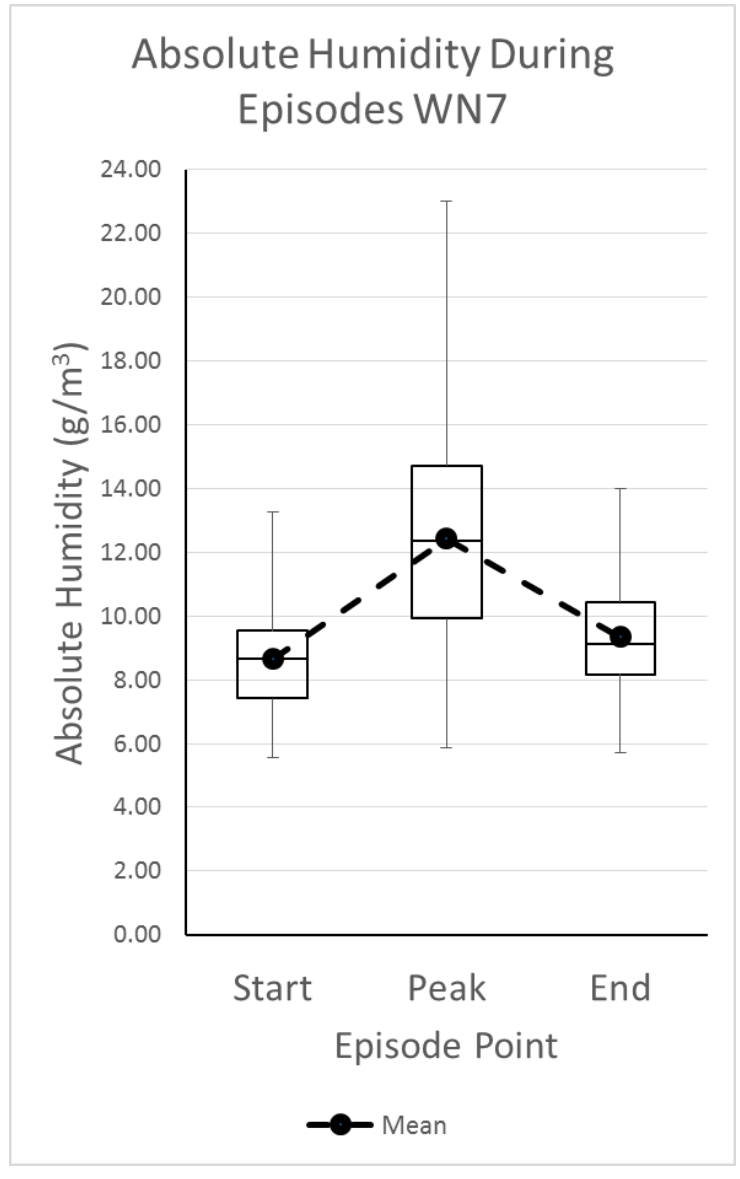

Figure 84 Average Absolute Humidity for Episodes in the Bathroom of House WN7
Every day had at least one moisture event take place in the bathroom of house WN7. The most common type of moisture event was an episode (Figure 84), with 104 episodes taking place over a period of 61 days. The most common time for episodes to take place was from 8.00pm - 12.00am (50/61) followed by $8.00 \mathrm{am}-12.00 \mathrm{pm}$ (37/61). Episodes also took place from 4.00pm - 8.00pm (13/61). Every day had at least one episode take place from $4.00 \mathrm{pm}-8.00 \mathrm{pm}$ and $8.00 \mathrm{pm}-$ 12.00am. Typical days were:

- one or more morning episodes, one or more evening episodes; and

- no morning episode, one or more evening episodes. 


\subsection{Results Summary WN8.1}

A large number of days had no moisture events take place in the first bathroom of house

WN8 (20/61). The most common type of moisture event was an episode (Figure 85), with 43

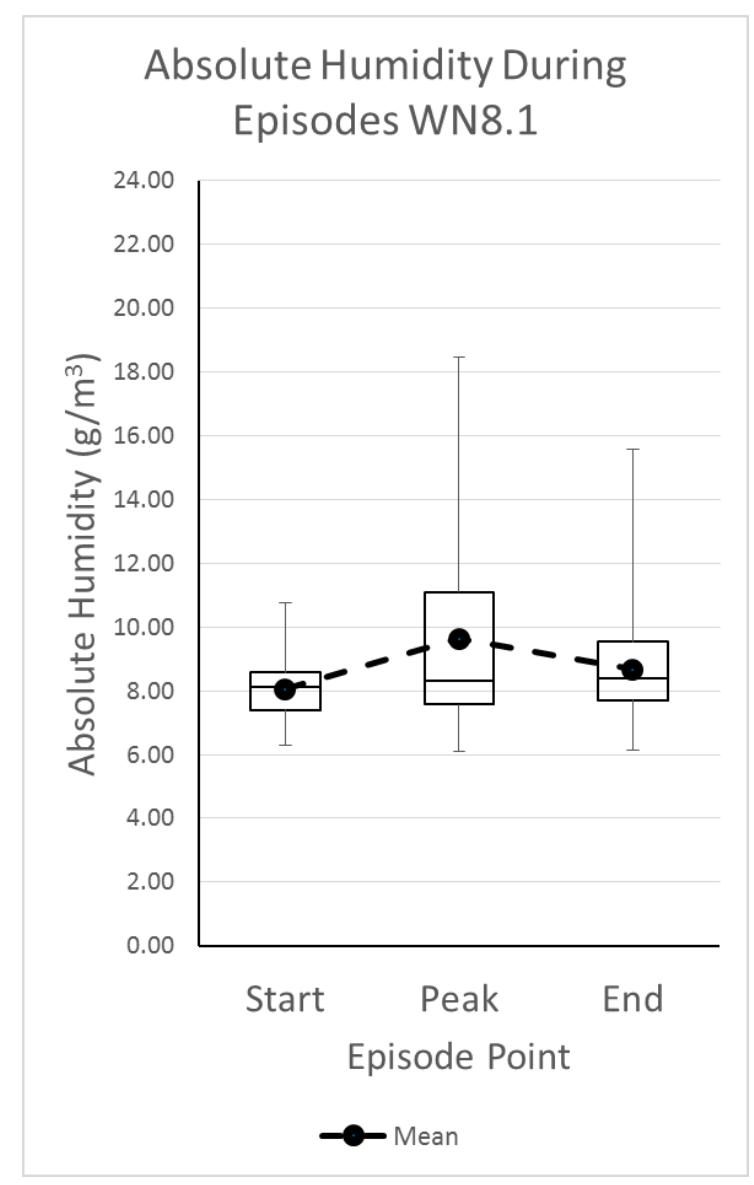

Figure 85 Average Absolute Humidity for Episodes in Bathroom WN8.1 episodes taking place over a period of 61 days. The most common time for episodes to take place was from 8.00am - 12.00pm (28/61) followed by 8.00pm - 12.00am $(11 / 61)$. Some of these evening episodes took place on a day where no episode took place during the morning $(6 / 61)$, but this did not happen frequently enough to determine whether there was a relationship between them. Typical days were:

- no moisture events;

- one or more morning episodes, no evening episode;

- one or more morning episodes, one or more evening episodes; and

- no morning episode, one or more evening episodes. 


\subsection{Results Summary WN8.2}

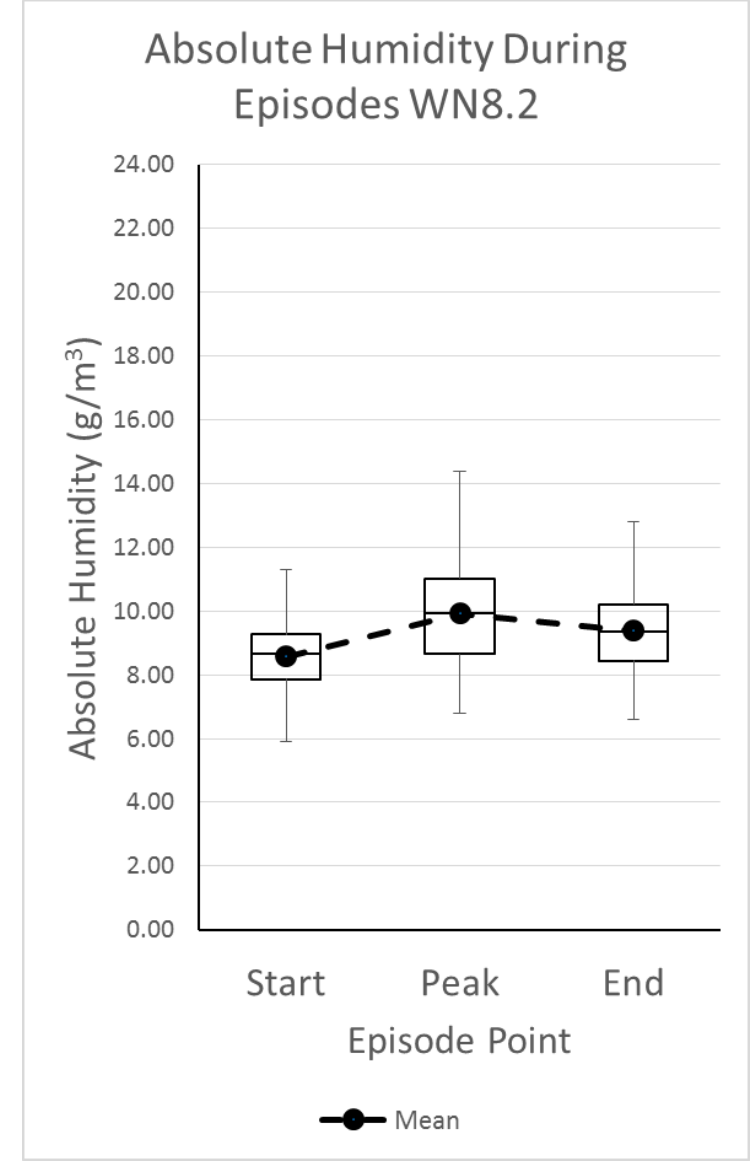

Figure 86 Average Absolute Humidity for Episodes in Bathroom WN8.2
Unlike WN8.1, every day had at least one

moisture event take place in the second bathroom of house WN8. The most common type of moisture event was an episode (Figure 86), with 86 episodes taking place over a period of 61 days. The most common time for episodes to take place was from $4.00 \mathrm{pm}$ 8.00pm (35/61) and 8.00pm - 12.00am (35/61). Every day had an episode take place during at least one of these periods. Episodes would also sometimes take place in the morning, most commonly from 4.00am 8.00am (10/61). Typical days were:

- one or more morning episodes, one or more evening episodes;

- no morning episode, one evening episode; and

- no morning episode, two evening episodes. 


\subsection{Results Summary WN9}

Every day had at least one moisture event take place in the bathroom of house WN9. The most common type of moisture event was an episode (Figure 87), with 152 episodes taking place over a period of 61 days. The most common times for these to take place in was from

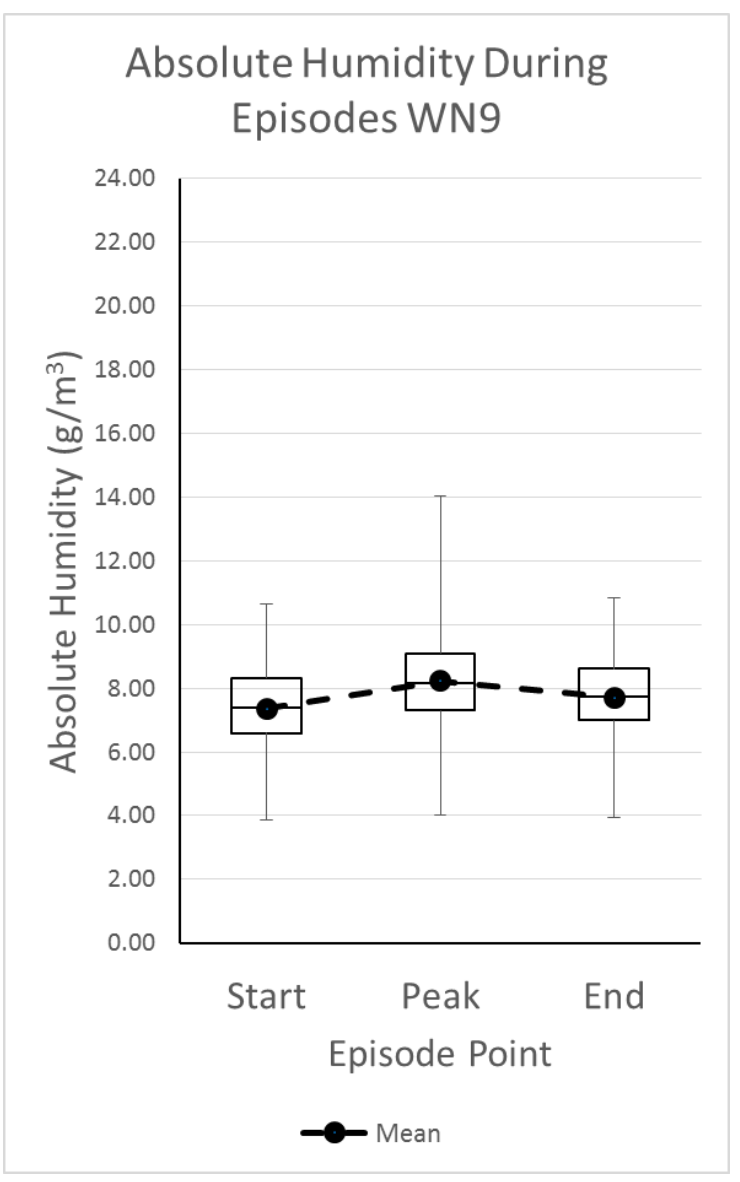

Figure 87 Average Absolute Humidity for Episodes in the Bathroom of House WN9 8.00pm - 12.00am (51/61) and 4.00am 8.00am (49/61). Every day had an episode take place during either $4.00 \mathrm{pm}-8.00 \mathrm{pm}$ $(18 / 61)$ or 8.00pm - 12.00am. Episodes were also common from 12.00am - 4.00am (15/61) and 8.00am - 12.00pm (18/61). However, these would often take place during days where there was an episode taking place from 4.00am - 8.00am. Typical days were:

- one morning episode, one evening episode;

- one morning episode, two evening episodes; and

- two morning episodes, one evening episode. 


\subsection{Results Summary WN10}

Only one day had no moisture events take place in the bathroom of house WN10 (1/58).

The most common type of moisture event was an episode (Figure 88), with 87 episodes taking place over a period of 58 days. The most common time for episodes to occur was

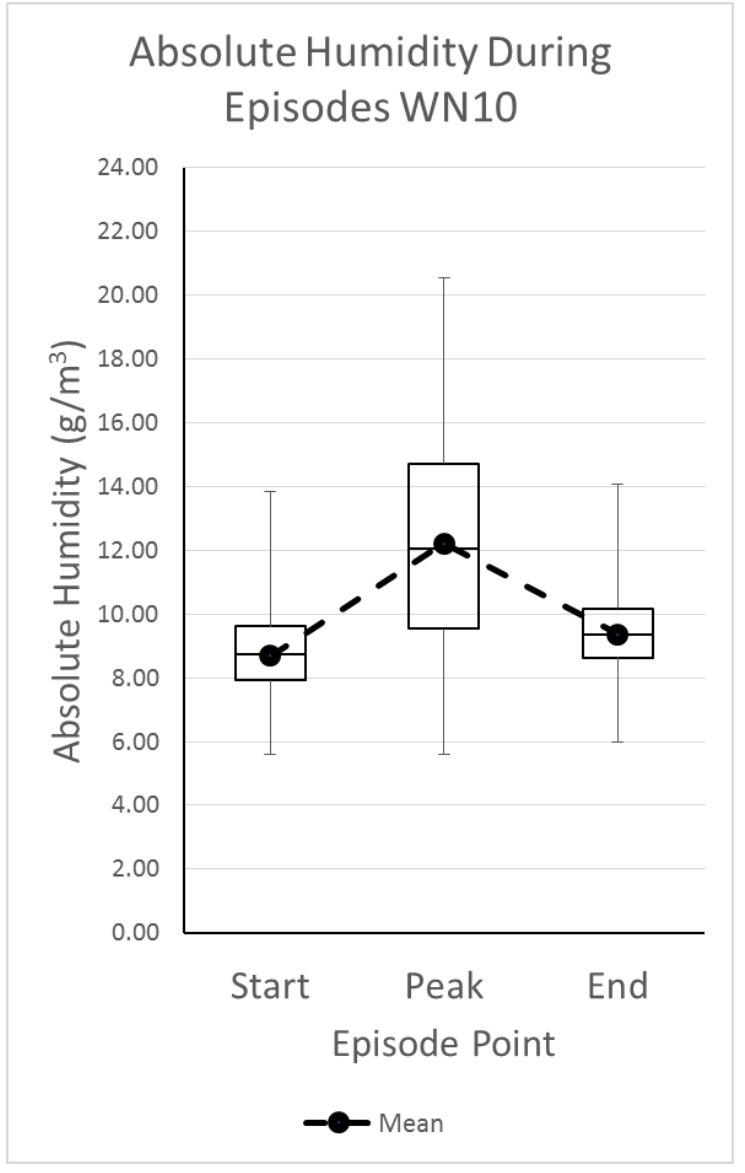

Figure 88 Average Absolute Humidity for Episodes in the Bathroom of House WN10 from 8.00pm - 12.00am (54/58). Episodes would also take place from 4.00pm - 8.00pm (4/58). Only one day did not have at least one episode take place during one of these two periods. The second most common time for episodes to take place was from 12.00am 4.00am (20/58). Episodes would occasionally take place during the remaining periods. However, no more than four episodes took place at the same time and never took place on the same day. Typical days were:

- one or more morning episodes, one or more evening episodes; and

- no morning episodes, one or more evening episodes. 


\section{Chapter 10: Groups and Trends in Data}

\subsection{Mean and Median Episodes for all Bathrooms}

This section brings together the data shown by bathroom in Chapter 9:. 

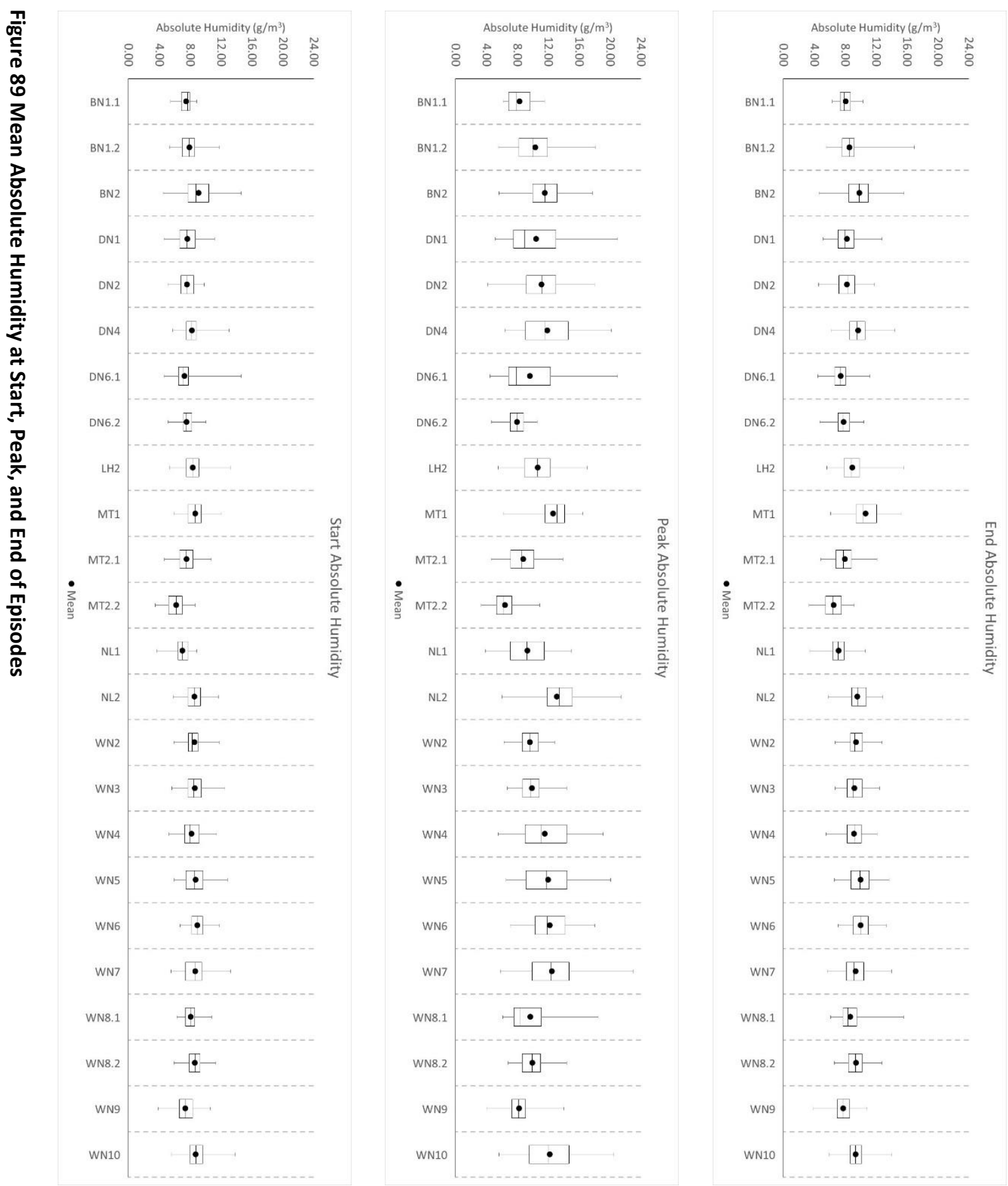
Moisture in New Zealand Bathrooms
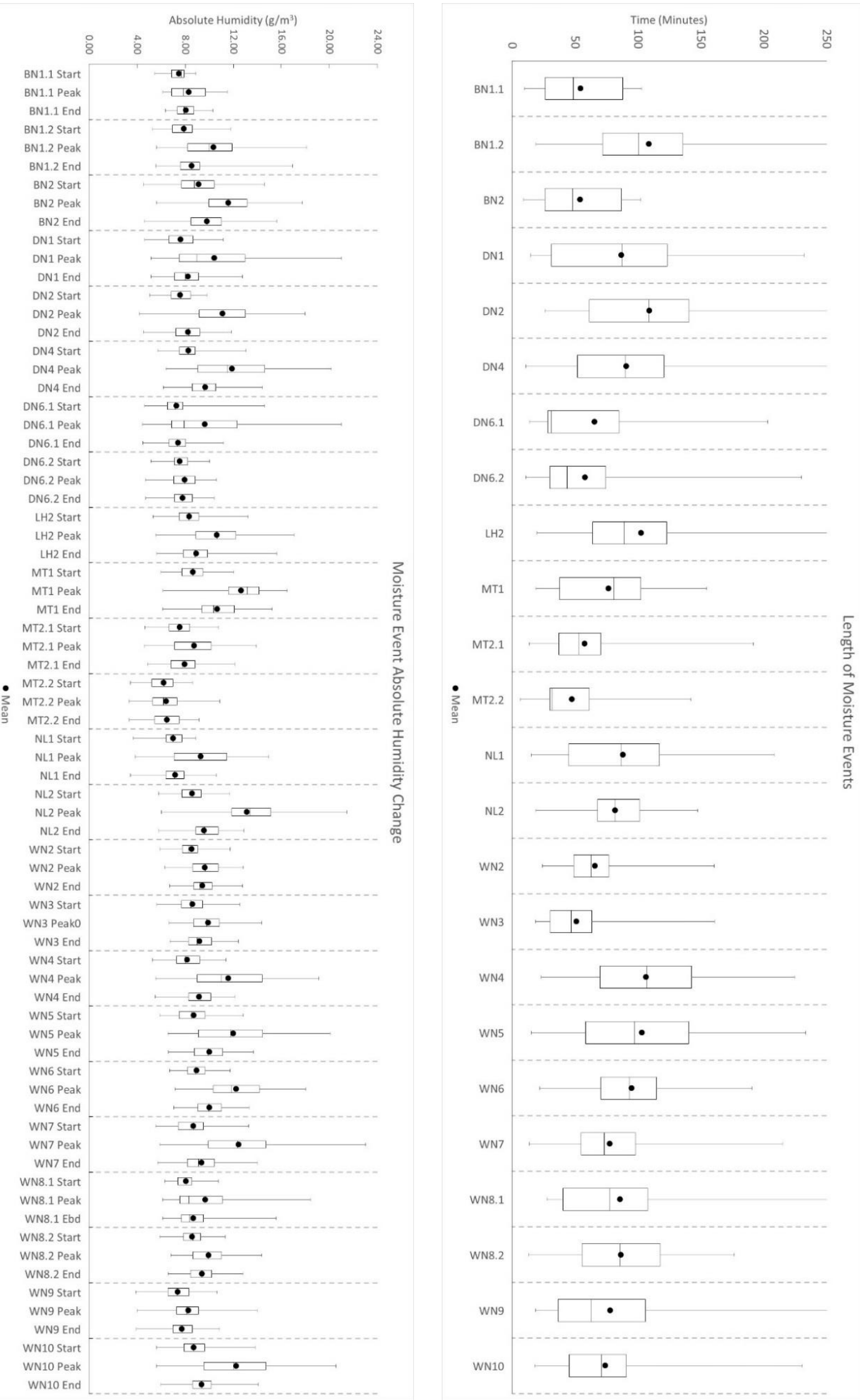
Figure 89 shows the mean and median absolute humidity at the start, peak, and end for episodes from each bathroom. A side-by-side comparison of how the absolute humidity changed in each bathroom is provided in Figure 90 alongside the mean and median length for these episodes. There was very little consistency between the episodes of each bathroom.

Some of the maximum values listed in Figure 90 were too large to be displayed alongside all other information (Table 17). This included all maximum lengths greater than 250 minutes. These were typically caused by moisture events beginning before the last moisture event had ended.

\begin{tabular}{|l|c|}
\hline \multicolumn{1}{|c|}{ House } & $\begin{array}{c}\text { Maximum Length } \\
\text { (Minutes) }\end{array}$ \\
\hline BN1.2 & 379 \\
\hline DN4 & 260 \\
\hline LH2 & 363 \\
\hline WN8.1 & 353 \\
\hline WN9 & 282 \\
\hline
\end{tabular}

Table 17 Large Maximum Episode Lengths 
Moisture in New Zealand Bathrooms
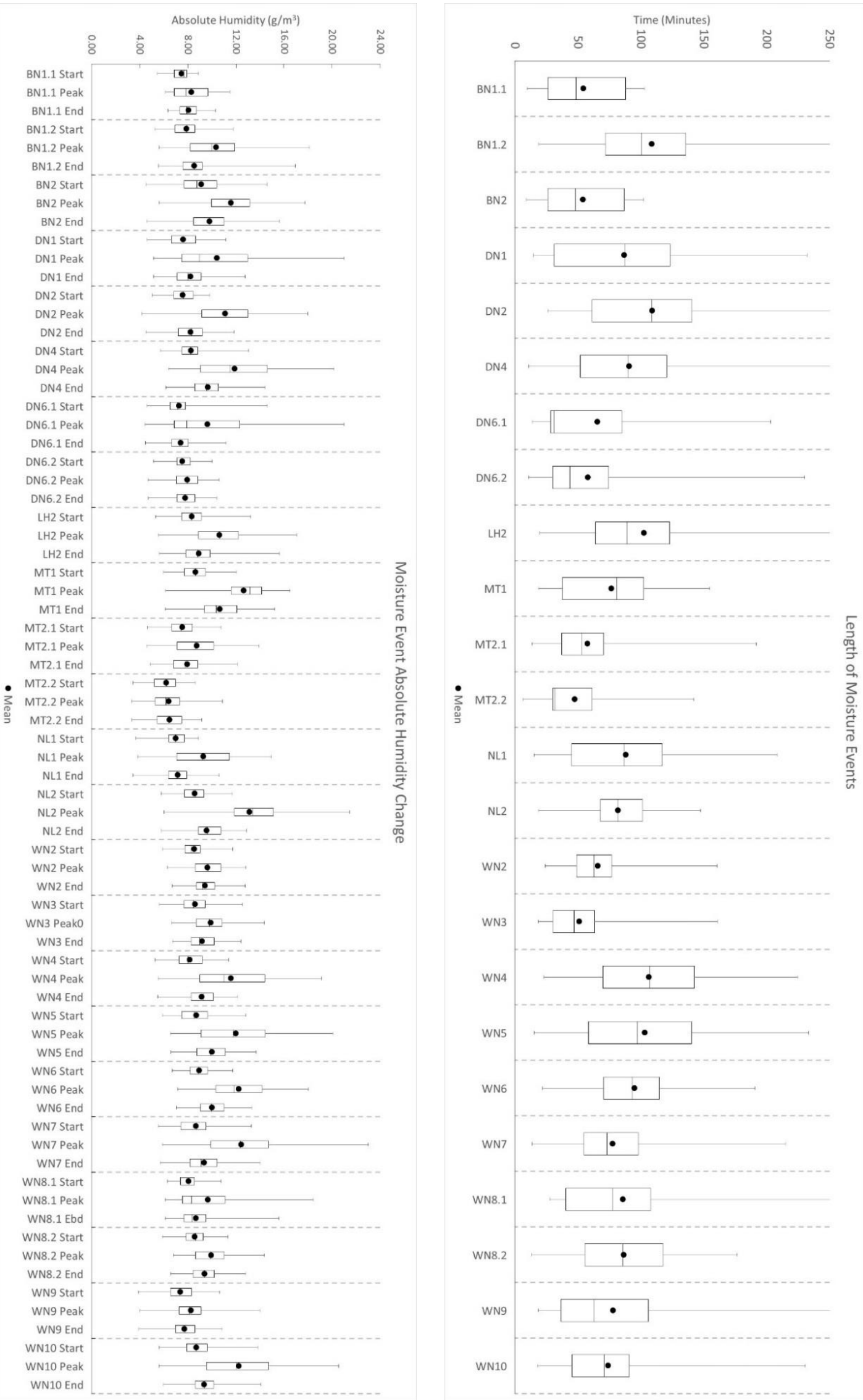


\subsection{Comparing Results to the Household Energy End-use} Project

\subsubsection{Showers/baths per day per House}

The number of episodes identified for each bathroom in Chapter 9: Summary of Individual Houses provides an indication of the number of showers and baths that took place in each household per day (Table 18). To calculate the average number of showers/baths per day in a household with two bathrooms, the total number of moisture generating episodes was determined and then divided by the total number of days minus the number of days where no moisture event took place.

\begin{tabular}{|c|c|c|c|c|c|c|}
\hline Bathroom & $\begin{array}{l}\text { Total } \\
\text { Period }\end{array}$ & $\begin{array}{c}\text { Total Days } \\
\text { with no } \\
\text { Moisture } \\
\text { Events }\end{array}$ & $\begin{array}{c}\text { Total } \\
\text { Episodes }\end{array}$ & $\begin{array}{c}\text { Showers/baths } \\
\text { per day }\end{array}$ & $\begin{array}{c}\text { Showers/baths } \\
\text { per day } \\
\text { (2 Bathrooms) }\end{array}$ & $\begin{array}{c}\text { Estimated } \\
\text { Showers/baths } \\
\text { per day }\end{array}$ \\
\hline BN1.1 & 61 & 17 & 9 & 0.20 & 2.39 & 2.39 \\
\hline BN1.2 & 61 & 2 & 132 & 2.24 & & 2.24 \\
\hline BN2 & 61 & 4 & 143 & 2.51 & & 2.51 \\
\hline DN1 & 61 & 0 & 109 & 1.79 & & 1.79 \\
\hline DN2 & 61 & 0 & 166 & 2.72 & & 2.72 \\
\hline DN4 & 61 & 1 & 128 & 2.13 & & 2.13 \\
\hline DN6.1 & 61 & 28 & 28 & 0.85 & 1.45 & 1.45 \\
\hline DN6.2 & 61 & 29 & 20 & 0.63 & & 0.63 \\
\hline LH2 & 61 & 2 & 131 & 2.22 & & 2.22 \\
\hline MT1 & 61 & 1 & 60 & 1.00 & & 1.00 \\
\hline MT2.1 & 61 & 6 & 103 & 1.87 & 2.45 & 2.45 \\
\hline MT2.2 & 61 & 26 & 32 & 0.91 & & 0.91 \\
\hline NL1 & 61 & 20 & 67 & 1.63 & & 1.63 \\
\hline $\mathrm{NL2}$ & 61 & 5 & 90 & 1.61 & & 1.61 \\
\hline WN2 & 55 & 21 & 34 & 1.00 & & 1.00 \\
\hline WN3 & 61 & 10 & 68 & 1.33 & & 1.33 \\
\hline WN4 & 61 & 5 & 79 & 1.41 & & 1.41 \\
\hline WN5 & 61 & 1 & 97 & 1.62 & & 1.62 \\
\hline WN6 & 61 & 6 & 75 & 1.36 & & 1.36 \\
\hline WN7 & 61 & 0 & 104 & 1.70 & & 1.70 \\
\hline WN8.1 & 61 & 20 & 43 & 1.05 & 2.11 & 2.11 \\
\hline WN8.2 & 61 & 0 & 86 & 1.41 & & 1.41 \\
\hline WN9 & 61 & 0 & 152 & 2.49 & & 2.49 \\
\hline WN10 & 58 & 1 & 87 & 1.53 & & 1.53 \\
\hline
\end{tabular}

Table 18 Estimated Shower/Bath Use per Day Based on Episodes Filtered for Typical Days 
This table excluded days with no moisture events to ensure that only days where the house was occupied were included. However, this meant that days where occupants were present but did not take a shower/bath would have been excluded, potentially inflating the number of showers/baths per day. Using episodes filtered for typical days may have further influenced results. Filtered episodes were only counted once per period, regardless of how many episodes occurred during that period. If multiple showers were taken over short period (such as from 6.00am - 8.00am) these would only count as one episode. This process also assumed that all episodes represent a shower or similar moisture source. Because of all this, Table 18 provides only an estimate of shower usage by house.

\begin{tabular}{|l|c|c|c|c|c|c|c|}
\hline Bathroom & $\begin{array}{c}\text { Total } \\
\text { Period }\end{array}$ & $\begin{array}{c}\text { Total Days } \\
\text { with no } \\
\text { Moisture } \\
\text { Events }\end{array}$ & $\begin{array}{c}\text { Total } \\
\text { Episodes }\end{array}$ & $\begin{array}{c}\text { Episodes } \\
\text { per Day }\end{array}$ & $\begin{array}{c}\text { Episodes per } \\
\text { Day } \\
\text { (2 Bathrooms) }\end{array}$ & $\begin{array}{c}\text { Estimated } \\
\text { Showers/baths } \\
\text { per day }\end{array}$ & $\begin{array}{c}\text { Average } \\
\text { Peaks per } \\
\text { Episode }\end{array}$ \\
\hline BN1.1 & 61 & 17 & 16 & 0.36 & 2.93 & $\mathbf{2 . 9 3}$ & 21.81 \\
\hline BN1.2 & 61 & 2 & 157 & 2.66 & & $\mathbf{2 . 6 6}$ & 4.82 \\
\hline BN2 & 61 & 4 & 514 & 9.02 & & $\mathbf{9 . 0 2}$ & 1.66 \\
\hline DN1 & 61 & 0 & 182 & 2.98 & & $\mathbf{2 . 9 8}$ & 1.47 \\
\hline DN2 & 61 & 0 & 104 & 1.70 & & $\mathbf{1 . 7 0}$ & 2.52 \\
\hline DN4 & 61 & 1 & 148 & 2.47 & & $\mathbf{2 . 4 7}$ & 1.47 \\
\hline DN6.1 & 61 & 28 & 77 & 2.33 & 3.73 & $\mathbf{3 . 7 3}$ & 3.75 \\
\hline DN6.2 & 61 & 29 & 46 & 1.44 & & $\mathbf{1 . 4 4}$ & 6.96 \\
\hline LH2 & 61 & 2 & 108 & 1.83 & & $\mathbf{1 . 8 3}$ & 1.55 \\
\hline MT1 & 61 & 1 & 69 & 1.15 & & $\mathbf{1 . 1 5}$ & 4.30 \\
\hline MT2.1 & 61 & 6 & 146 & 2.65 & 3.82 & $\mathbf{3 . 8 2}$ & 1.51 \\
\hline MT2.2 & 61 & 26 & 64 & 1.83 & & $\mathbf{1 . 8 3}$ & 4.72 \\
\hline NL1 & 61 & 20 & 70 & 1.71 & & $\mathbf{1 . 7 1}$ & 4.23 \\
\hline NL2 & 61 & 5 & 97 & 1.73 & & $\mathbf{1 . 7 3}$ & 2.77 \\
\hline WN2 & 55 & 21 & 39 & 1.15 & & $\mathbf{1 . 1 5}$ & 8.38 \\
\hline WN3 & 61 & 10 & 88 & 1.73 & & $\mathbf{1 . 7 3}$ & 3.16 \\
\hline WN4 & 61 & 5 & 100 & 1.79 & & $\mathbf{1 . 4 7}$ & 3.56 \\
\hline WN5 & 61 & 1 & 104 & 1.73 & & $\mathbf{2 . 1 1}$ & 10.71 \\
\hline WN6 & 61 & 6 & 81 & 1.47 & & 3.50 & 3.58 \\
\hline WN7 & 61 & 0 & 129 & 2.11 & & & \\
\hline WN8.1 & 61 & 20 & 80 & 1.95 & 3.00 & & \\
\hline
\end{tabular}




\begin{tabular}{|l|c|c|c|c|c|c|c|}
\hline WN8.2 & 61 & 0 & 103 & 1.69 & & $\mathbf{1 . 6 9}$ & 2.55 \\
\hline WN9 & 61 & 0 & 286 & 4.69 & & $\mathbf{4 . 6 9}$ & 2.26 \\
\hline WN10 & 58 & 1 & 121 & 2.12 & & $\mathbf{2 . 1 2}$ & 2.02 \\
\hline
\end{tabular}

Table 19 Estimated Showers/baths per day Based on all Episodes

Rather than using the filtered episodes identified for typical days (7.5 Filtering Moisture Events for Typical Days), Table 19 represents all identified episodes. However, this includes episodes regardless of the impact they had on the indoor environment. This increases the chances of episodes that did not represent a shower or similar moisture source being included.

Table 18 and Table 19 both provide an indication of the number of showers taking place in each house. Table 18, based on filtering episodes by size, length, and time of day indicated that the mean number of showers/baths per day per house was $1.74 \pm 0.25$. Table 19 , based on every moisture event that qualified as an episode, indicated that the mean number of showers/baths per day per house was $2.52 \pm 0.66$. According to self-reported shower usage as part of the HEEP report, the number of showers/baths per day per house was $2.5 \pm 0.1$ (Camilleri et al., 2010). While it may appear that Table 19 provides a good indicator of the number of showers taken per day per house, as it closely matches the results of the HEEP report, there was too much variance between the houses for this result to be meaningful. However, it does show that the results of this study appear to match the results of the HEEP report.

This further highlights the difference between the criteria used to identify episodes for each purpose. Filtering the episodes to find typical days meant that many episodes which potentially represented large changes in the indoor environment were ignored or grouped together. Typical days are useful for providing information about regular moisture events and for providing context for episode analysis but excludes too much data to be used for analysing individual episodes.

\subsubsection{Shower Length}

The mean and median length of episodes can be used as an indicator of shower length (Figure 92). This assumes that every episode represents a shower or similar moisture source. The shower length is estimated based on the amount of time moisture was actively 
Moisture in New Zealand Bathrooms

being introduced to the space. This is measured from the episode start to the first peak, effectively measuring the length of time for the first increase in each episode. 
Alister Stubbe

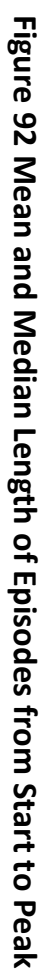

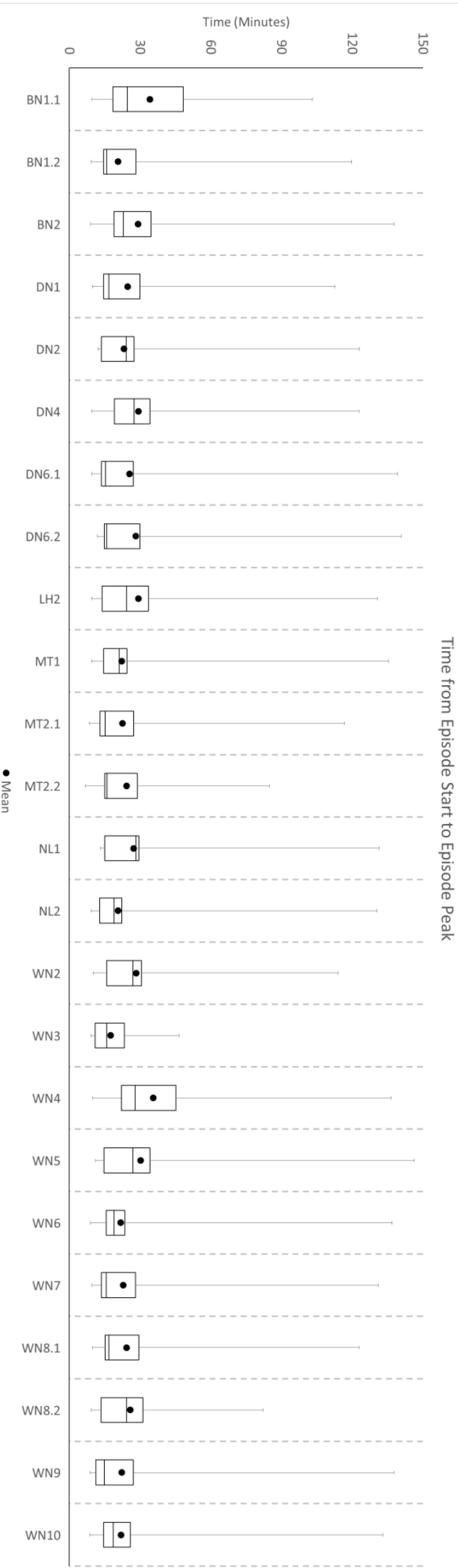

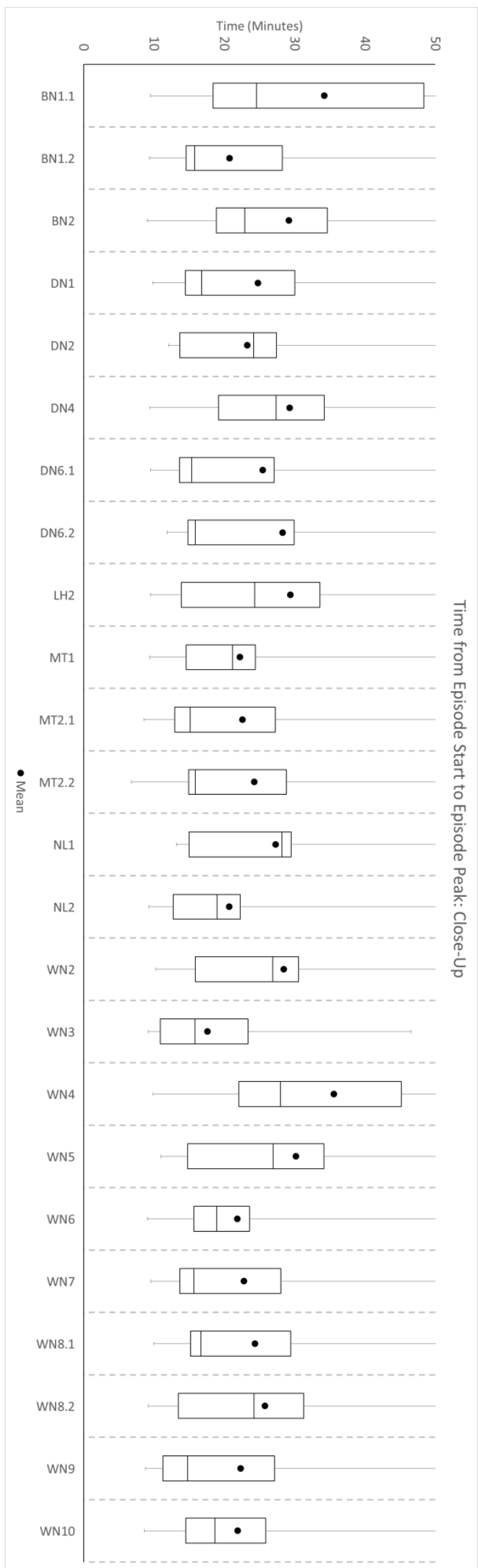


Some of the longest episodes took almost 150 minutes to reach their peak (Figure 92). These episodes were unlikely to represent the short burst of moisture a shower would introduce, distorting the mean length. The median length was used instead. Based on this, the average length of time from episode start to peak was $21 \pm 1.95$ minutes. However, this time does not necessarily reflect the length of showers.

Moisture events were counted as beginning from the measurement before the first measurement to increase/decrease by a rate of 3\% or higher, as discussed in 6.3.1 Examples of Different Moisture Event Levels. For episodes that began suddenly this had no impact. In these cases the first measurement with an increase of $3 \%$ or more would be when the shower or other moisture source was already underway. The measurement before it (the first measurement in the moisture event) reflected the indoor environment directly before the shower or other moisture source was turned on. However, some episodes started with a low rate of change that escalated over time. For example, if the change in absolute humidity began with a small increase that still registered as a moisture event, such as sink taps being turned on, before the shower began the length of time between the start and peak would increase. Because of this, the length of time from episode start to episode peak (21 minutes) is likely longer than the real shower length. The results of the HEEP report appear to confirm this, with the self-reported average shower duration was $9.5 \pm 0.2$ minutes, although shower durations varied widely (Camilleri et al., 2010).

\subsection{Using the Gathered Data for Analysis}

To better understand how moisture events differ between New Zealand houses, results were grouped and sorted according to measurable qualities. The main two qualities that can be analysed in the available data are changes in absolute humidity during an episode and changes in episode length as described in 7.3.2 Calculating Average Moisture Events.

\subsubsection{Grouping by Absolute Humidity}

Groups are based on the differences and similarities between episodes in each house. Unlike the filtered episodes used for typical days, this did not have restrictions based on the size or length of episode. All events that met the general definition of an episode were included (7.4.1 Episode). This was any episode with an increase in absolute humidity that 
reached one (or more) peaks, followed by a decrease. Measuring a single type of event will ensure that conclusions drawn from averages will be relevant to the indoor environment. This mitigates the potential issue of the "average event" reflecting the midpoint between two entirely different events rather than the average of a single type of event. As described in 7.3.2 Calculating Average Moisture Events, this used the mean and median absolute humidity calculated at the start, the peak(s), and the end of the episodes.

\title{
10.3.2 Grouping by Event Length
}

\begin{abstract}
Analysing how long the average event lasts for in each house provides information on both the typical conditions experienced in these bathrooms and additional context for analysing changes in absolute humidity. This was calculated from the start point of an episode to the end point. As the start and end points were based on the rate of change rather than the level of absolute humidity, this length will reflect the period moisture is rapidly being added and removed from the indoor environment rather than the length of time the moisture introduced by the episode is still present.
\end{abstract}

\subsubsection{Accurately Representing Episodes}

In some cases a new episode began before the original episode ends. If this was not a regular occurrence it may represent an outlier, disproportionately influencing the mean measurement of event length. Similar outliers could be created in a number of ways, such as if a daily episode containing multiple peaks is treated as several smaller episodes one day. To prevent these outliers from affecting results, the median episode information was used.

\subsubsection{Missed Episodes}

Using a clear and consistent definition for episodes (an increase registering as a moisture event directly followed by a decrease registering as a moisture event) is vital for providing data that can be compared to other houses. However, this does present the possibility of not including events that should be included. For example, in some events the absolute humidity may plateau after increasing, remaining at its peak for several minutes. As no change is taking place during this period, it would not be counted as an episode, instead registering as two separate events: an increase and a decrease.

One step that was taken to prevent this from happening was to include the measurement taken directly before and after a moisture event as being part of that event. This means that 
Moisture in New Zealand Bathrooms

for an episode to be counted as two separate events there would need to have been at least 5 measurements taken while the absolute humidity remained at its peak. With measurements commonly being taken 10 or 15 minutes apart, this would mean that the episode would have to remain at its peak for an hour before being counted as two different events.

\subsection{Taking Starting Levels of Absolute Humidity into Account}

10.4.1 Measuring Changes in Absolute Humidity from Start to Peak of Episode

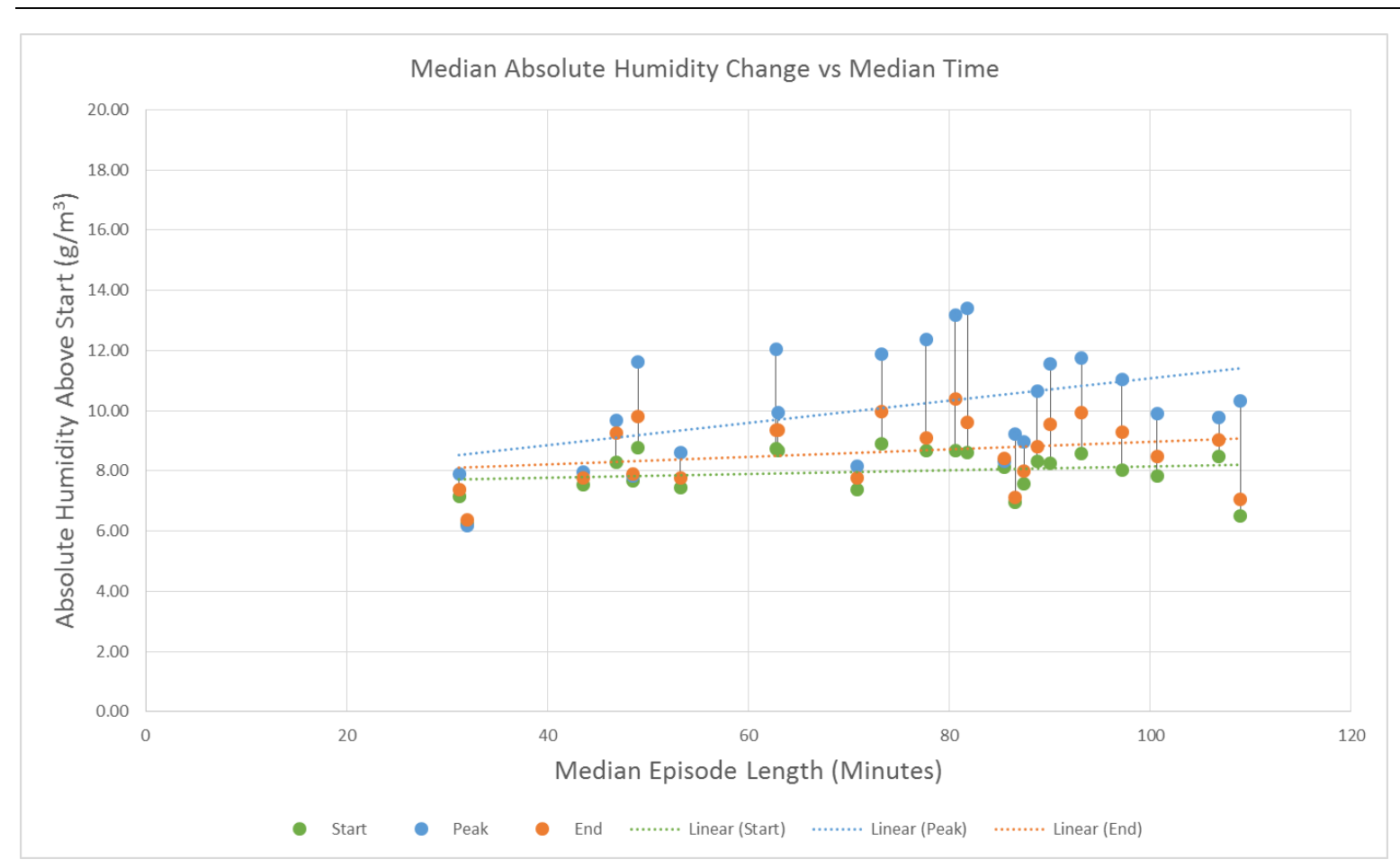

Figure 93 Median Absolute Humidity at each Point of Episode compared to Mean Time

161

Groups and Trends in Data 


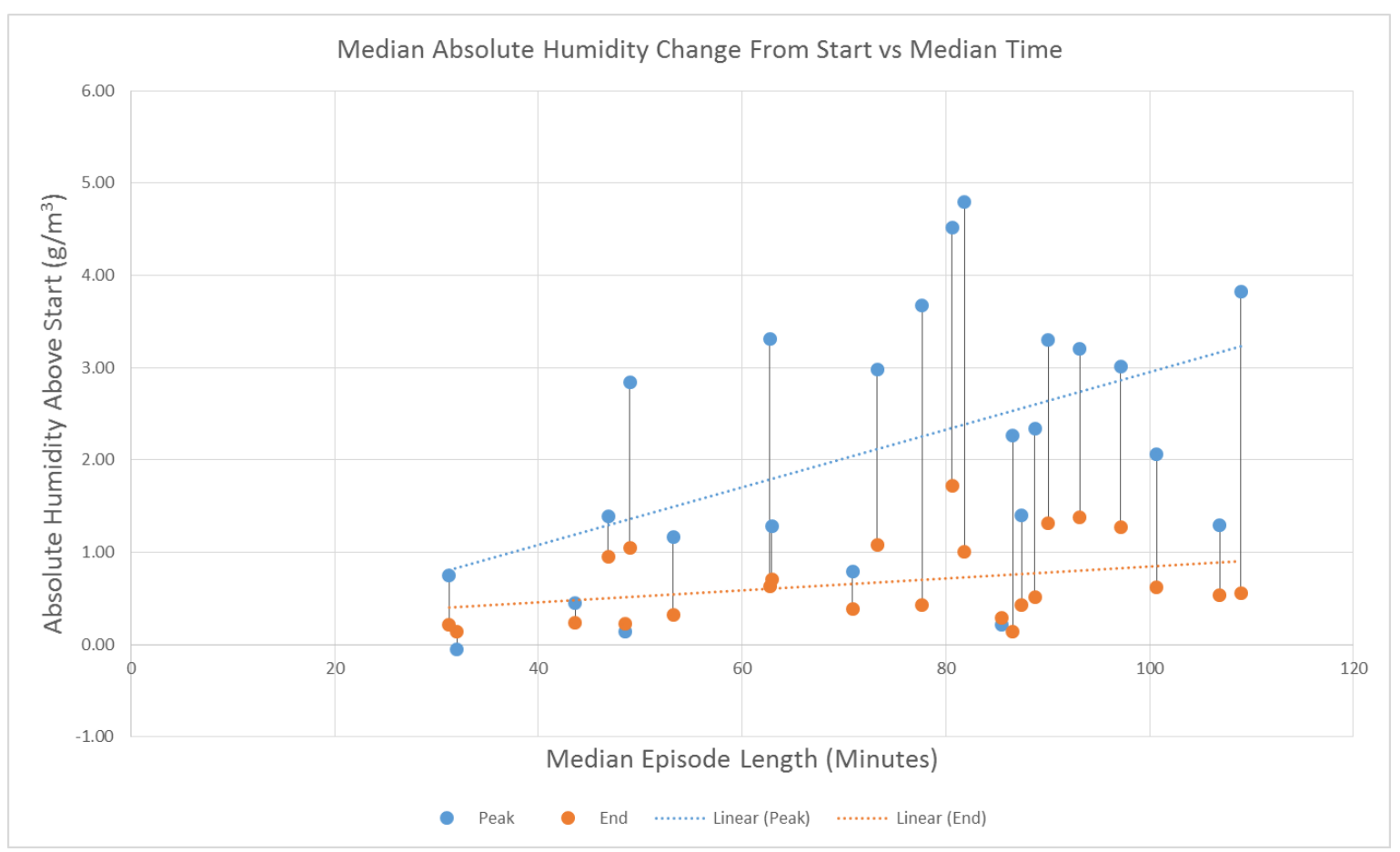

Figure 94 Bathrooms Corrected for Start Absolute Humidity compared to Median Time

The analysis is based on the change that takes place during the episode, meaning that the absolute humidity in the bathroom at the start of each episode is not important. Figure 93 shows the median absolute humidity at the start, peak, and end of episodes for each house. Figure 94 shows the change in median absolute humidity in all bathrooms at the peak and end of episode compared to the start. As the median absolute humidity at the peak was calculated using all peaks that took place during the episode, the median absolute humidity was lower at the peak than the end in rare cases, such as when a peak would frequently happen soon after the start of a moisture event. Bathrooms are listed in the order of median episode length in both graphs. All the changes compared to the start absolute humidity were within a range of $5 \mathrm{~g} / \mathrm{m}^{3}$.

This is a useful method of comparing episodes. It demonstrates which houses experienced the greatest change in absolute humidity during an episode rather than which houses experienced the highest peaks in absolute humidity. This makes it easier to analyse the impact different factors have on moisture events, such as the number of bathrooms and the bathrooms size.

This method does have issues. There is a limit to how much moisture air is capable of holding before reaching saturation. Not taking the start absolute humidity into account 
ignores the differences in an episode where the absolute humidity increases from $7 \mathrm{~g} / \mathrm{m}^{3}-$ $10 \mathrm{~g} / \mathrm{m}^{3}$ to an episode where it increases from $9 \mathrm{~g} / \mathrm{m}^{3}-12 \mathrm{~g} / \mathrm{m}^{3}$. Calculating the exact impact this has on results is beyond the scope of this research but was taken into consideration during the analysis. This also highlights one of the issues of using absolute humidity rather than relative humidity, discussed further in 11.2 Absolute Humidity.

\subsubsection{Median Peak Absolute Humidity}

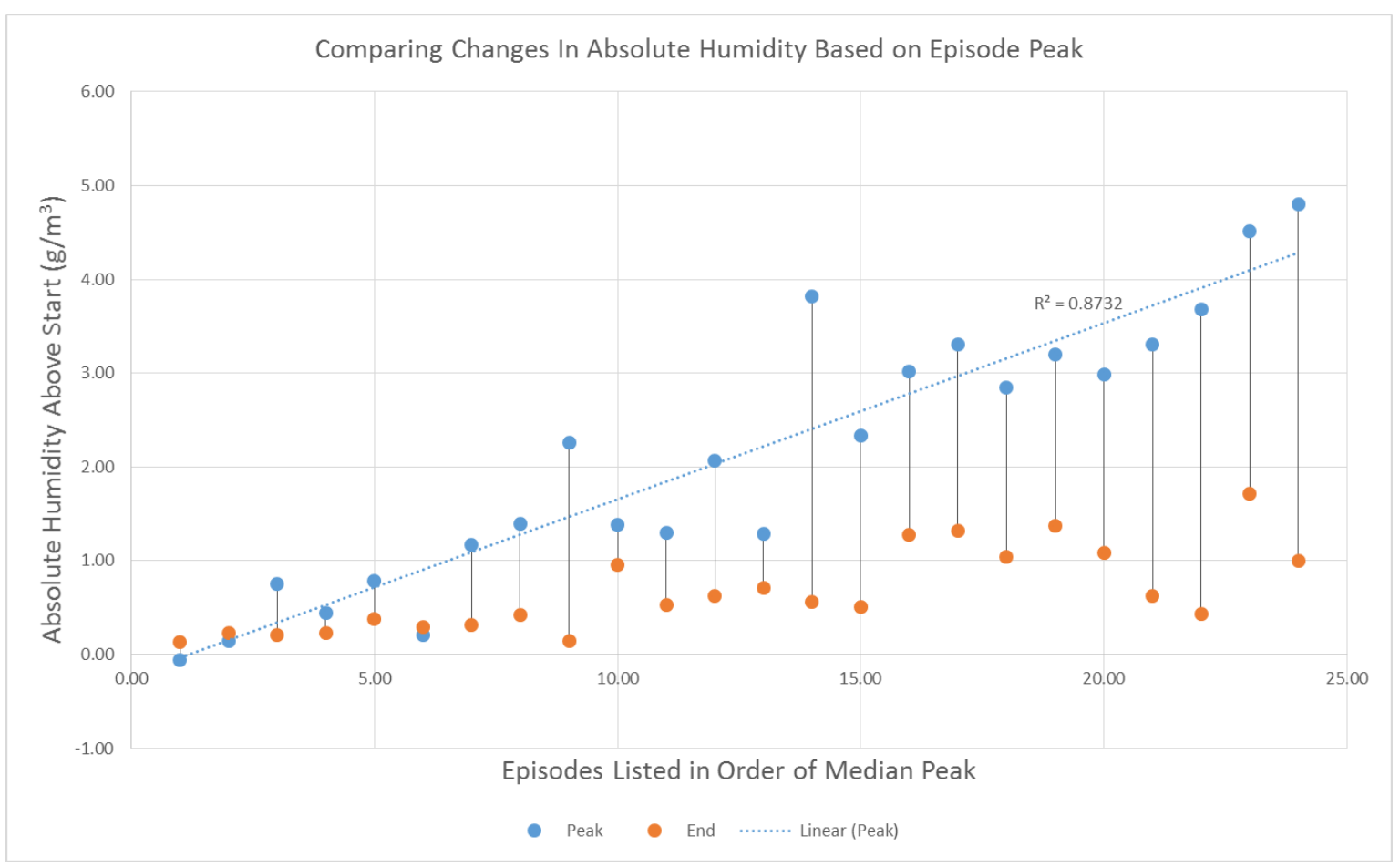

Figure 95 Bathrooms Corrected for Start Absolute Humidity, Sorted by Peak Absolute Humidity

Figure 95 shows the change in median absolute humidity from the start to the peak and end of episodes for each house, sorting them by the median peak absolute humidity. If the start absolute humidity had a large impact on the results, Figure 95 would be expected to show no clear relationship between the change in median absolute humidity between the start and peak, and the order of absolute humidity at the peak of episodes. This is not the case. As can be seen in Figure 95, as the median peak absolute humidity of each episode increases, on average the change from the start to the peak also increases.

This suggests that a greater increase in the median absolute humidity from the start to the peak of an episode takes place for an episode that experienced a larger peak in absolute humidity. This appears to confirm an idea discussed in the initial data test (6.6.2 Comparison 
to Weather), where the amount of moisture introduced during an episode may be so large that it reduces the impact of the starting absolute humidity (affected by factors such as the weather).

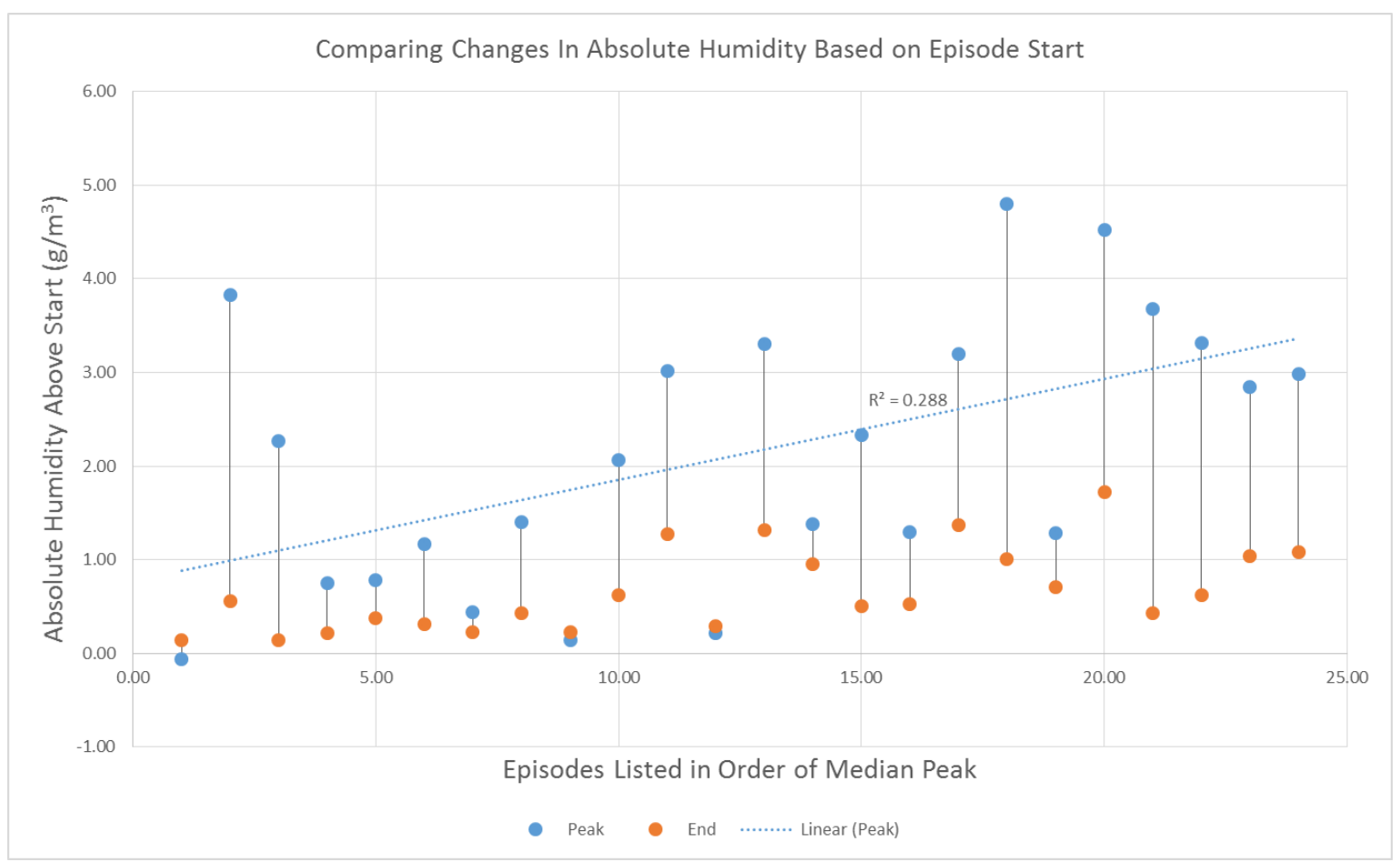

Figure 96 Bathrooms Corrected for Start Absolute Humidity, Sorted by Start Absolute Humidity

Figure 96 further confirms this. It shows the same information as Figure 95, but sorted according to the median start absolute humidity of episodes in each bathroom. There is no clear relationship between the median start absolute humidity and the change in median absolute humidity from the start to the peak $\left(R^{2}=0.288\right)$. This seems to confirm that the absolute humidity at the start of an episode had little impact on the subsequent change in absolute humidity. 


\subsection{Number of Bathrooms}

\subsubsection{One Bathroom}

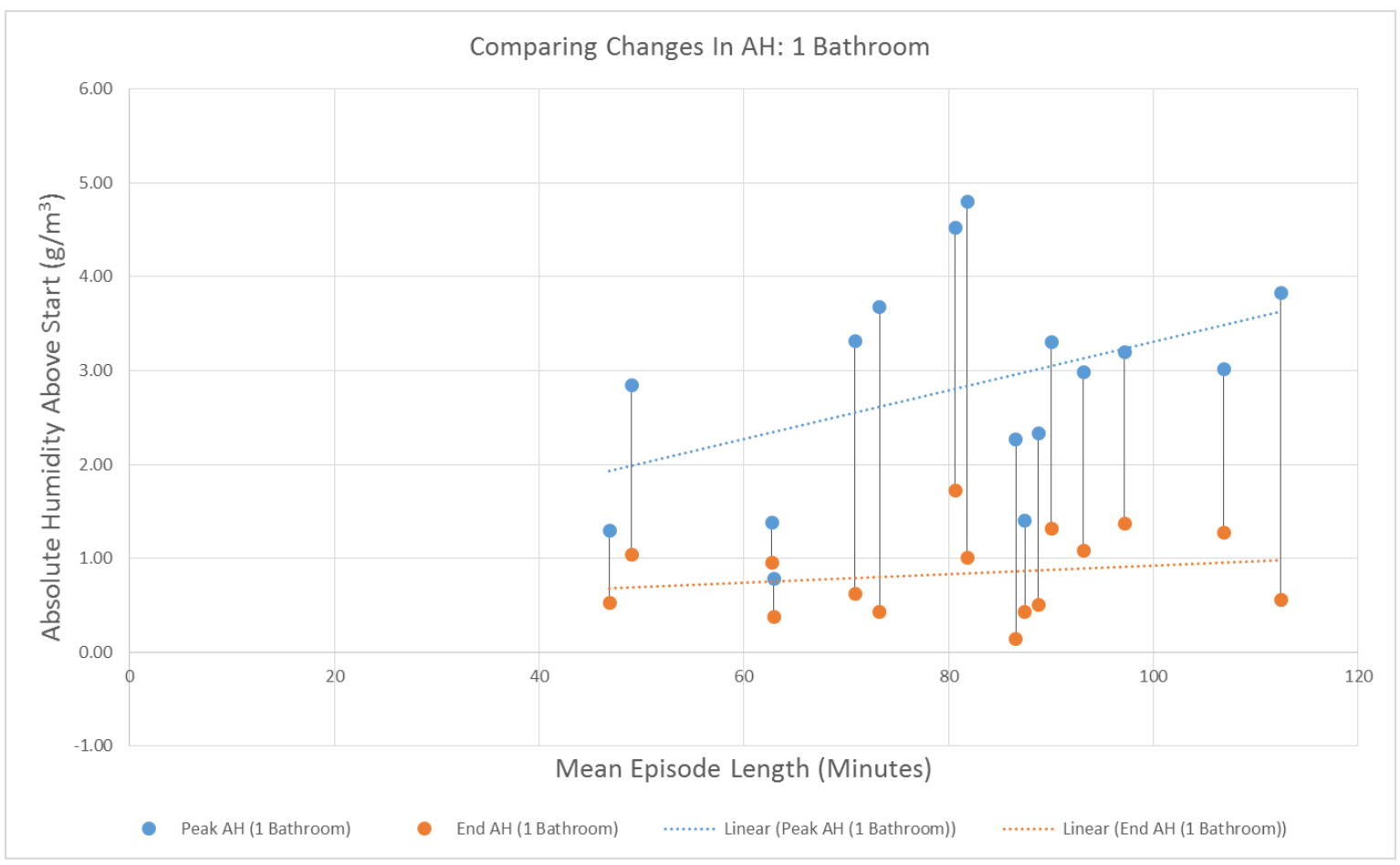

Figure 97 Changes in Absolute Humidity Compared to Start, Houses with One Bathroom

Figure 97 shows the changes in absolute humidity and length recorded in houses with only one bathroom. While there was little consistency in the changes in absolute humidity, in every bathroom the peak absolute humidity was higher than the end absolute humidity. There is no obvious correlation between mean episode length and the difference between the peak and end absolute humidity. On average, the peak absolute humidity was $10.97 \mathrm{~g} / \mathrm{m}^{3}$. This was an average increase of $2.81 \mathrm{~g} / \mathrm{m}^{3}$ from the start to peak of an episode. 


\subsubsection{Two Bathrooms}

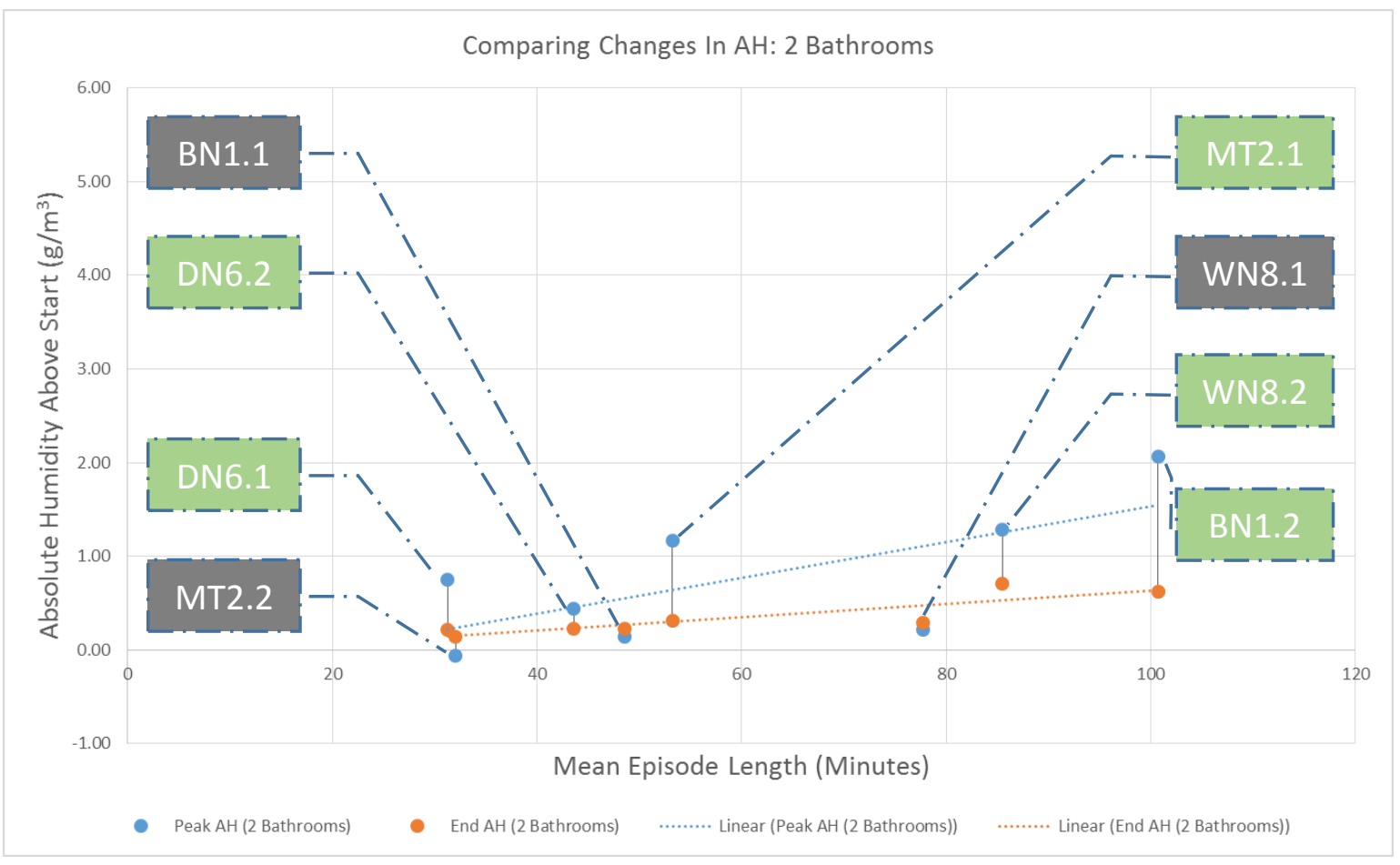

Figure 98 Changes in Absolute Humidity Compared to Start, Houses with Two Bathrooms

Figure 98 shows the changes in absolute humidity and length recorded in houses with two bathrooms. Unlike houses with only one bathroom, in some bathrooms the absolute humidity at the end of an episode was higher than the peak (bathrooms shown in grey). The definition of an episode required that each episode contained an increase followed by a decrease. This meant that for the end absolute humidity to be higher than the peak absolute humidity, the episode would either need multiple peaks or to begin with an increase and end with a decrease (with an increase followed by a decrease between them). This occurred in three bathrooms (BN1.1, MT2.2, and WN8.1), all from different houses, making it likely that this was a result limited to houses with multiple bathrooms.

In houses with two bathrooms the average peak absolute humidity across both bathrooms was lower than in houses with one bathroom. The average peak absolute humidity for all houses with two bathrooms was $8.33 \mathrm{~g} / \mathrm{m}^{3}$. This was an increase of $0.75 \mathrm{~g} / \mathrm{m}^{3}$ from the start to peak of an episode, almost a quarter of the increase experienced in bathrooms from houses with only one bathroom. 


\subsubsection{The Impact of a Second Bathroom}

A total of four of the analysed houses had a second bathroom: BN1, DN6, MT2, and WN8. In three of these houses the number of identified episodes in the second bathroom varied significantly from the first. For all houses, one of the bathrooms experienced a smaller change in median absolute humidity between the start and peak of episodes. As the bathroom numbers were assigned randomly, it did not matter whether the bathroom that experienced a smaller change was the first bathroom (e.g. BN1.1) or the second bathroom (e.g. BN1.2).

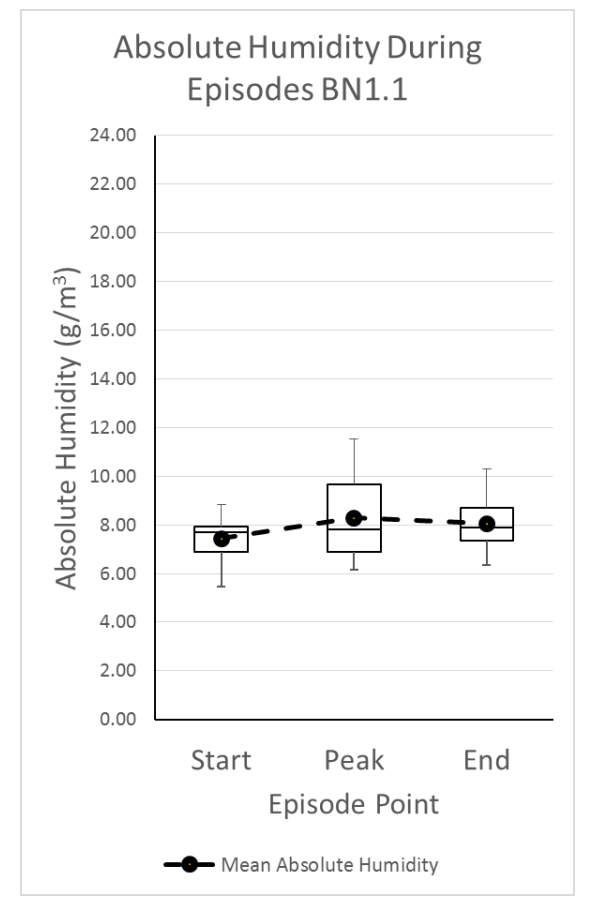

Total unfiltered episodes: 16

Typical days with no moisture events: 17

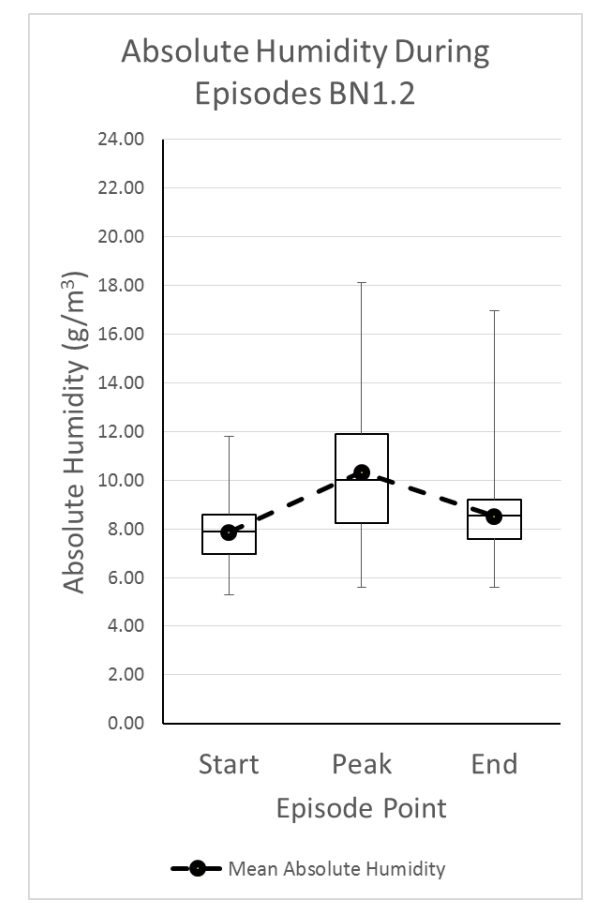

Total unfiltered episodes: 157

Typical days with no moisture events: 2

Figure 99 House BN1 Bathroom Comparison 
Alister Stubbe

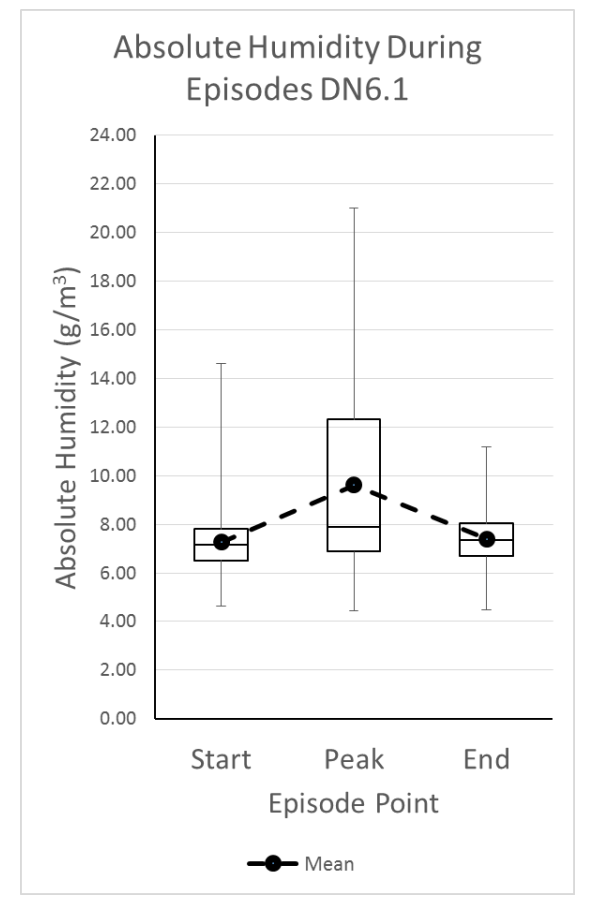

Total unfiltered episodes: 77

Typical days with no moisture events: 28

Figure 100 House DN6 Bathroom Comparison

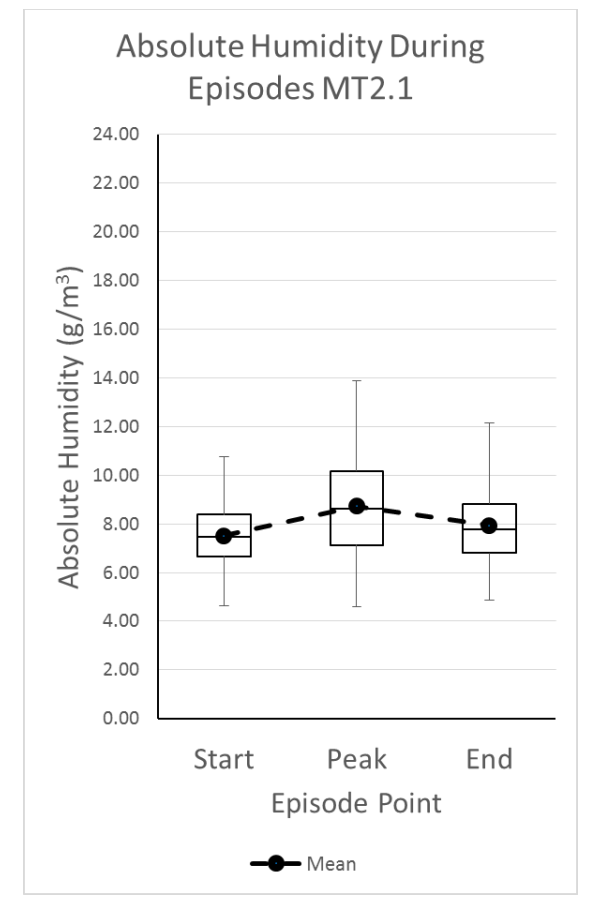

Total unfiltered episodes: 146

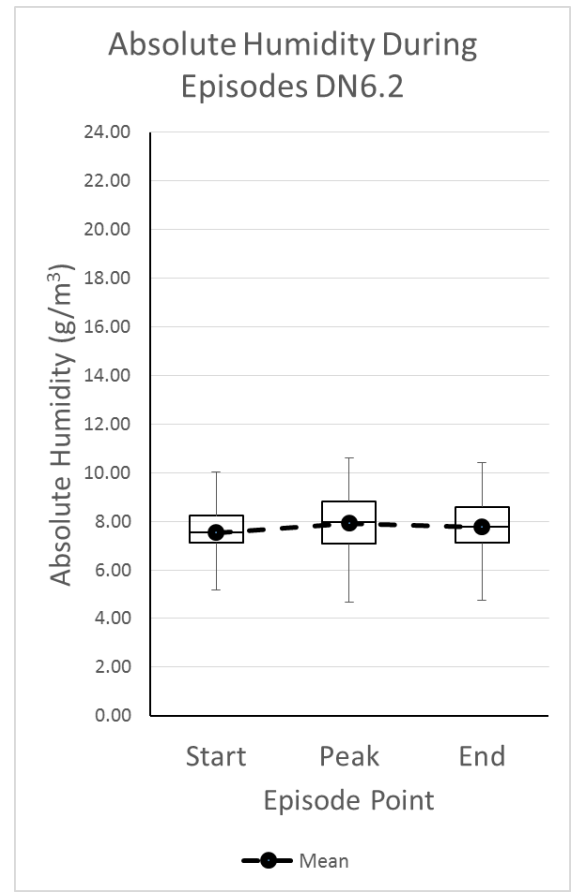

Total unfiltered episodes: 46

Typical days with no moisture events: 29

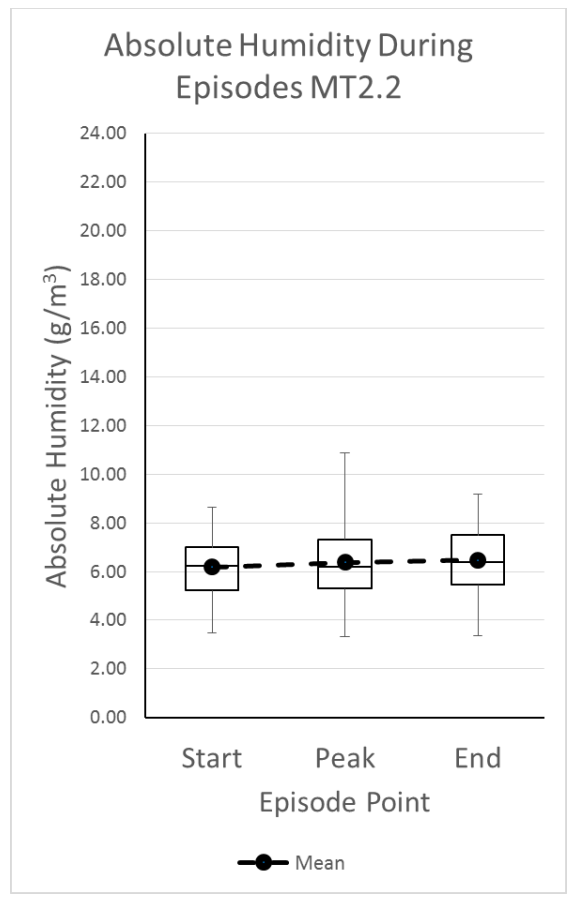

Total unfiltered episodes: 64 
Typical days with no moisture events: 6

Figure 101 House MT2 Bathroom Comparison

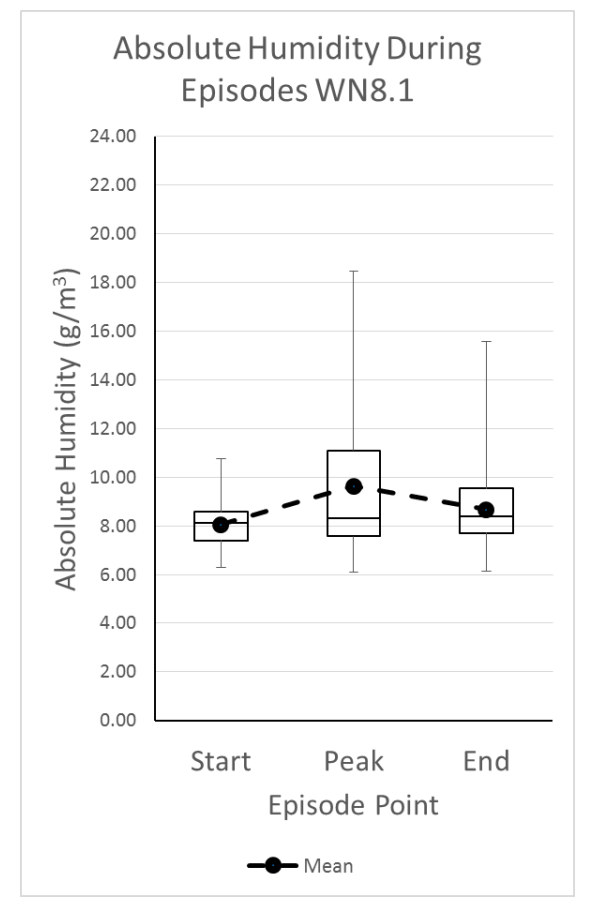

Total unfiltered episodes: 80

Typical days with no moisture events: 20

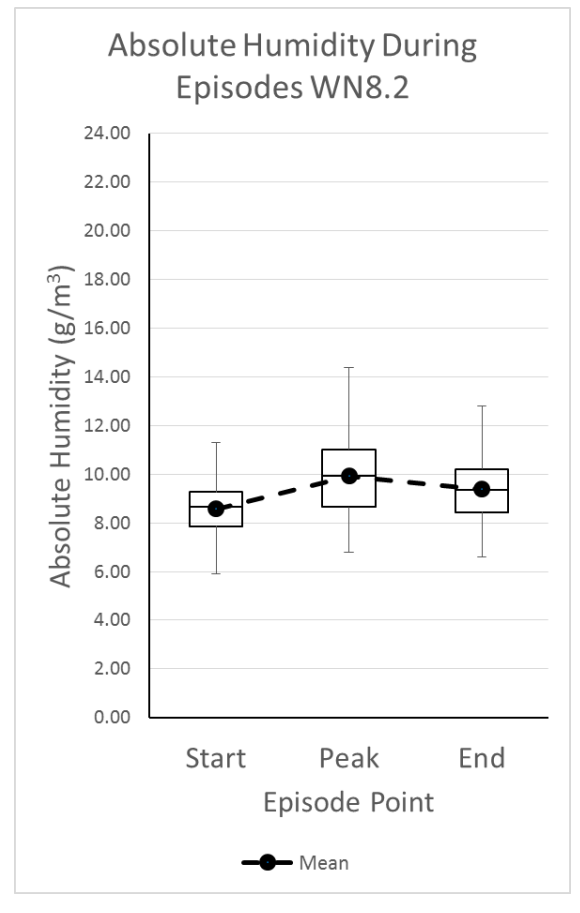

Total unfiltered episodes: 103

Typical days with no moisture events: 0

Figure 102 House WN8 Bathroom Comparison

Unlike the other houses, in house WN8 (Figure 102) the mean absolute humidity followed a similar pattern for both bathrooms. The bathroom which experienced the greatest change in mean absolute humidity, WN8.1, experienced the smallest change in median absolute humidity. This indicates that while large episodes did take place, they were far rarer than in the other bathroom.

This pattern of the median absolute humidity remaining at similar levels throughout episodes for one bathroom is consistent with the idea that occupants play a key role in controlling moisture (2.2 Ventilation). In houses with two bathrooms, it seems that one bathroom will act as the 'main bathroom' and see more consistent use. The other bathroom may be less available, such as if it is designed as an ensuite bathroom or on the second floor. In these situations, this second bathroom is likely to be used less often. The number of 
unfiltered episodes recorded in each bathroom appears to show that this was the case, with the bathroom that experienced smaller episodes also having fewer episodes.

Assuming that the design of both bathrooms is similar, a greater gap between periods of use could result in more air changes taking place before the next moisture source is turned on, resulting in a less saturated environment that can absorb a greater quantity of moisture. This would also explain why the change in absolute humidity from the start to the peak was lower, as having a second bathroom could result in the main bathroom being used less often, leaving more time between episodes.

Alternatively, with two bathrooms available for use, one bathroom may be more likely to be used for showers or baths. This would result in the episodes taking place in each bathroom to reflect entirely different types of moisture events. A bathroom where no showers or baths were taken would have insignificant changes in the absolute humidity take place compared to the flood of moisture a shower introduces to the indoor environment. However, when a shower or bath was taken this second bathroom would still experience similar maximum levels of absolute humidity during episode peaks. This may be the case in bathroom WN8.1, where the median and mean represent two very different situations.

\subsubsection{Excluding Bathrooms from Further Analysis}

Because of how different these 'other' bathrooms behaved, they were removed from the other groups created for analysing the results (BN1.1, DN6.2, MT2.2, and WN8.1). The 'main' bathroom was still included, but it is important to note that episodes that take place in these bathrooms may be influenced by the presence of a second bathroom. 


\subsection{Bathroom Area}

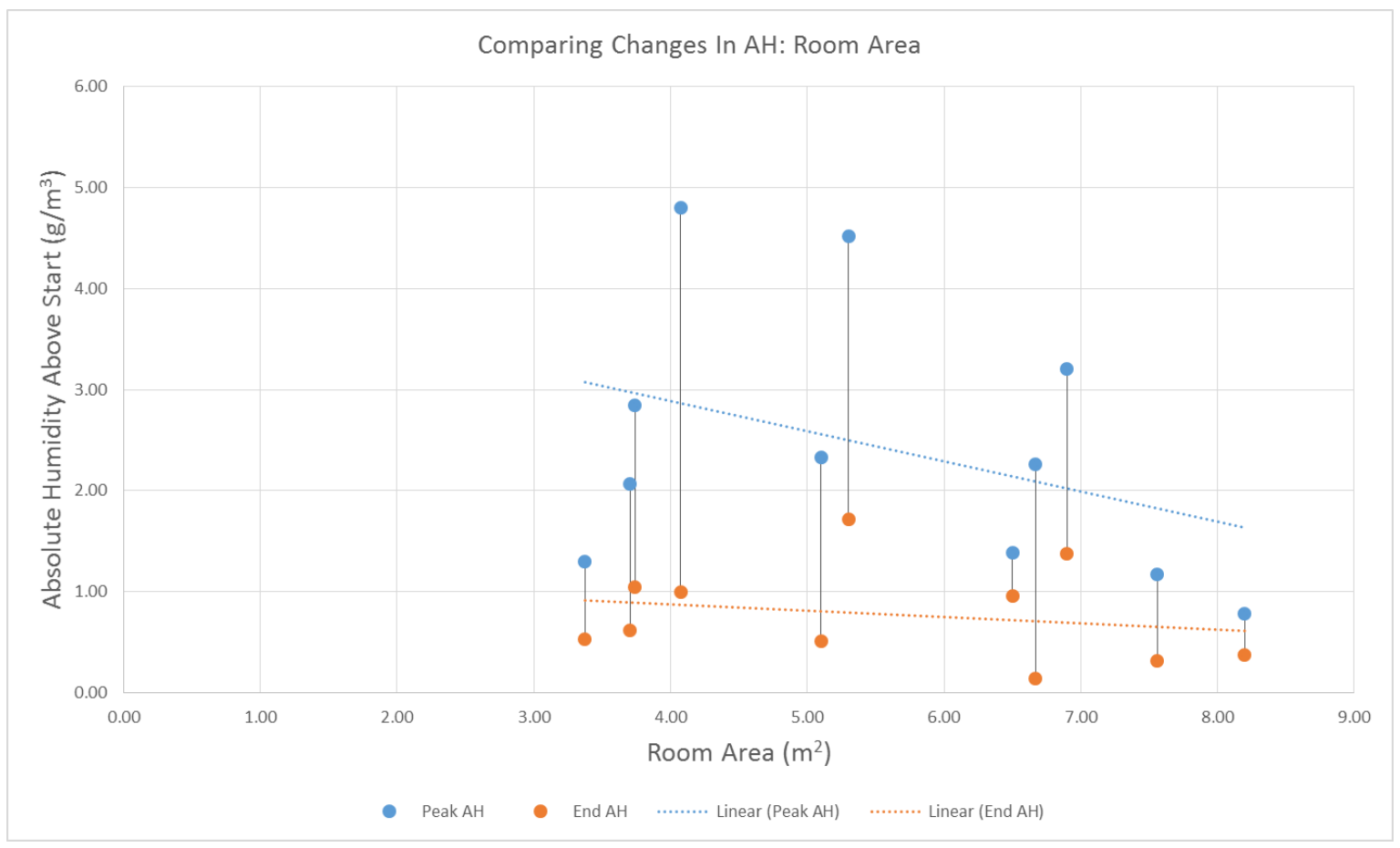

Figure 103 Changes in Absolute Humidity Compared to Start vs Floor Area

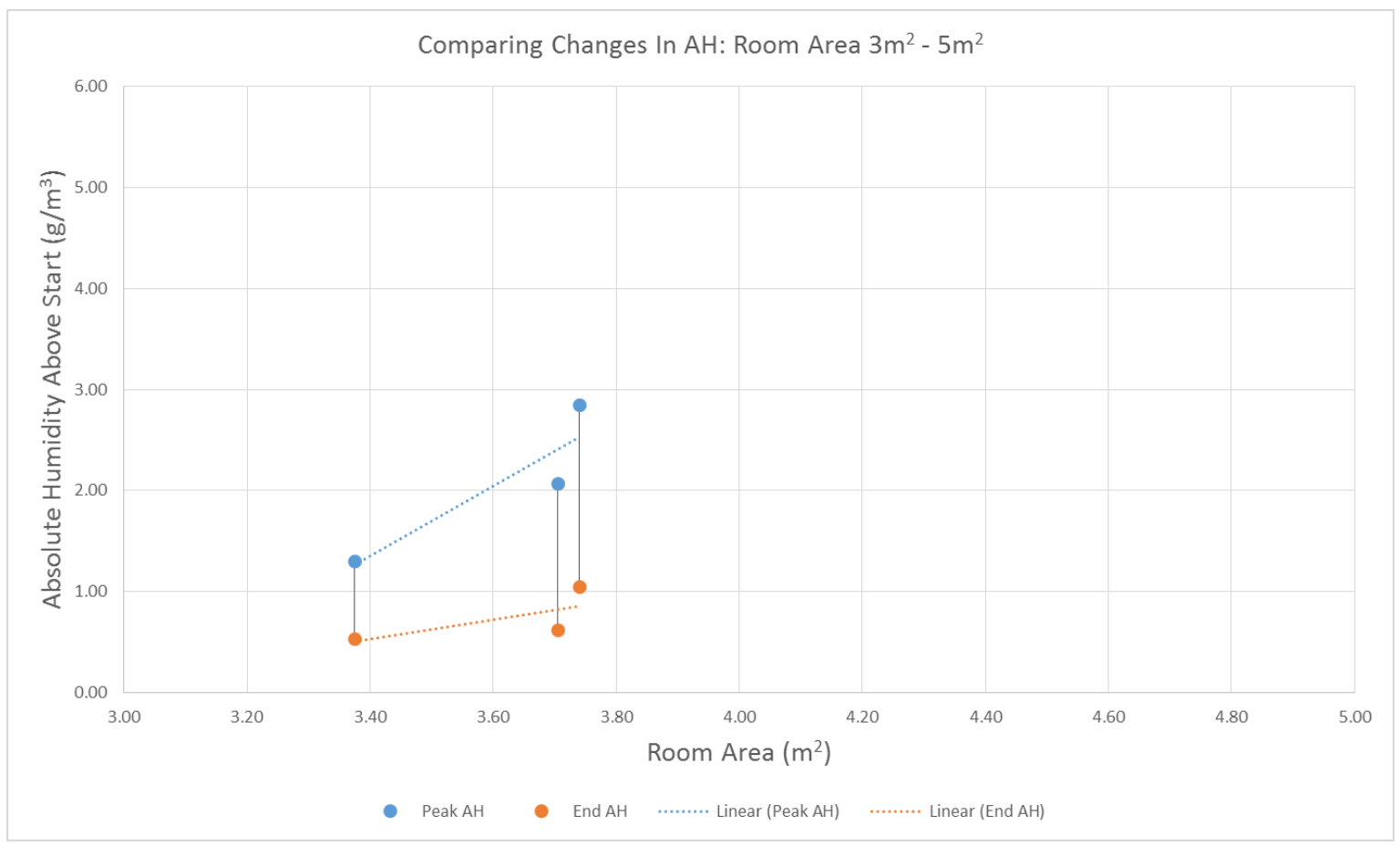

Figure 104 Changes in Absolute Humidity Compared to Start, $3 m^{2}-5 m^{2}$

171

Groups and Trends in Data 


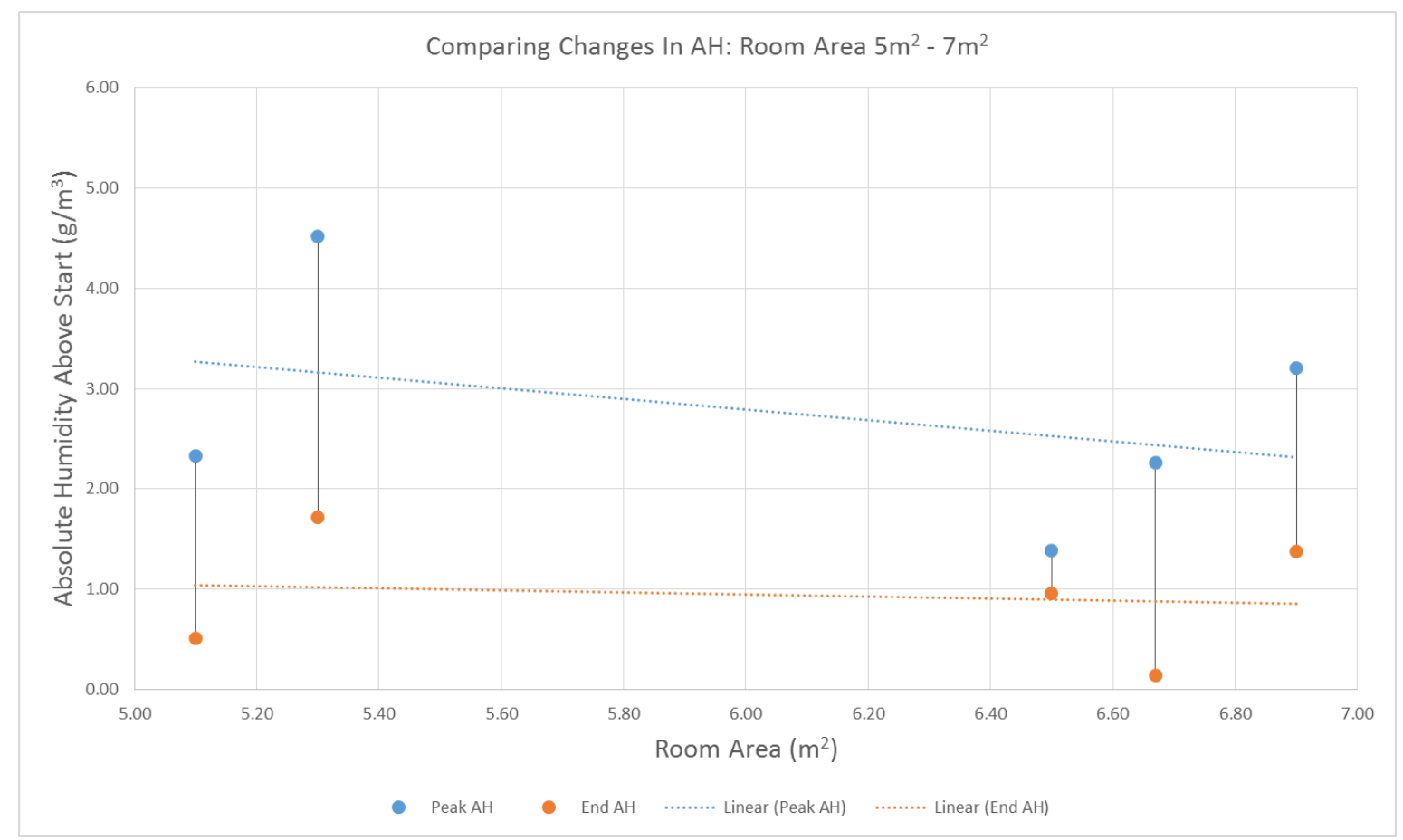

Figure 105 Changes in Absolute Humidity Compared to Start, $5 m^{2}-7 m^{2}$

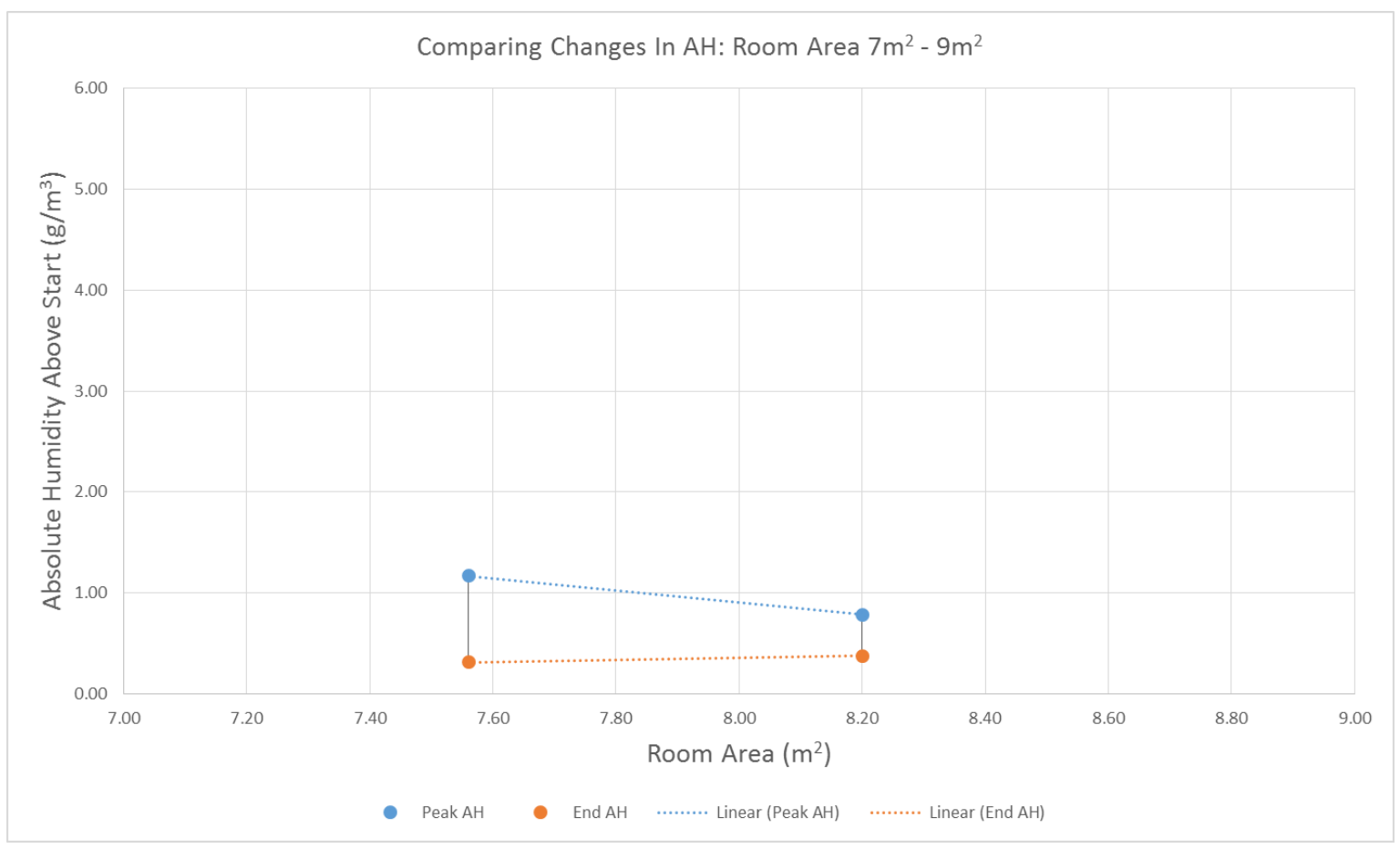

Figure 106 Changes in Absolute Humidity Compared to Start, $7 m^{2}-9 m^{2}$

Not all houses analysed in this study had the bathroom dimensions available. The bathrooms that did have this information did not include the height, only the length and 
width. It was assumed that all the bathrooms had the same height. This meant that the total volume of the bathroom was based on the floor area.

It could be expected that the absolute humidity would be proportional to the room size. Absolute humidity is measured in grams of water per cubic metre; so increasing the volume of the bathroom means that more moisture must be introduced to achieve the same quantity of moisture per cubic metre. For a similar length and moisture emission event, it would be expected that a larger bathroom would have a lower increase in absolute humidity from the start to the peak of an episode.

Most of the analysed bathrooms follow this trend (Figure 103). The largest bathrooms measured (Figure 106), MT2.1 $\left(7.5 \mathrm{~m}^{2}\right)$ and WN9 $\left(8.2 \mathrm{~m}^{2}\right)$, had the smallest increases in absolute humidity from the start of an episode to the peak: $1.17 \mathrm{~g} / \mathrm{m}^{3}$ and $0.79 \mathrm{~g} / \mathrm{m}^{3}$ respectively. As expected, bathrooms with a floor area between $5 \mathrm{~m}^{2}$ and $7 \mathrm{~m}^{2}$ (Figure 105), LH2 $\left(5.1 \mathrm{~m}^{2}\right), \operatorname{MT} 1\left(5.3 \mathrm{~m}^{2}\right), \mathrm{WN} 2\left(6.5 \mathrm{~m}^{2}\right), \mathrm{NL} 1\left(6.7 \mathrm{~m}^{2}\right)$, and WN5 $\left(6.9 \mathrm{~m}^{2}\right)$, had larger increases in absolute humidity from the start of an episode to the peak, with an average increase of $2.74 \mathrm{~g} / \mathrm{m}^{3}$.

This trend did not continue for the smallest bathrooms (Figure 104), NL2 (4.1 $\mathrm{m}^{2}$ ), BN2 $\left(3.7 \mathrm{~m}^{2}\right), \mathrm{BN} 1.2\left(3.7 \mathrm{~m}^{2}\right)$, and WN3 $\left(3.4 \mathrm{~m}^{2}\right)$. The smaller the bathroom, the smaller the increase from the start of the episode to the peak: $4.80 \mathrm{~g} / \mathrm{m}^{3}, 2.84 \mathrm{~g} / \mathrm{m}^{3}, 2.07 \mathrm{~g} / \mathrm{m}^{3}$, and $1.30 \mathrm{~g} / \mathrm{m}^{3}$ respectively.

There are several possible reasons for why this might be the case. These smaller bathrooms may have smaller moisture sources (such as a smaller shower) that introduce less moisture to the space. Alternatively, the smaller volume of air means that the same amount of ventilation as a larger room would replace all the air in the space in a shorter amount of time. This would result in the moisture being removed more quickly, potentially reducing the peak absolute humidity. Finally, this may be caused by the occupants. As established, a smaller room would be expected to reach high levels of absolute humidity. This may cause discomfort to occupants using the room (such as if the room became filled with steam during a shower). This may encourage the occupants to use ventilation, either by opening the window, turning on a fan, or both. The bathroom of NL2, which had the largest increase from the start of the episode to the peak, was also the only bathroom in the group that did 
not have mechanical ventilation (there was no data available on whether the bathroom of WN3 had mechanical ventilation). More information is required to determine the cause.

\subsection{Typical Days}

Note that second bathrooms (from houses with two bathrooms) were removed.

\subsubsection{Unoccupied Periods}

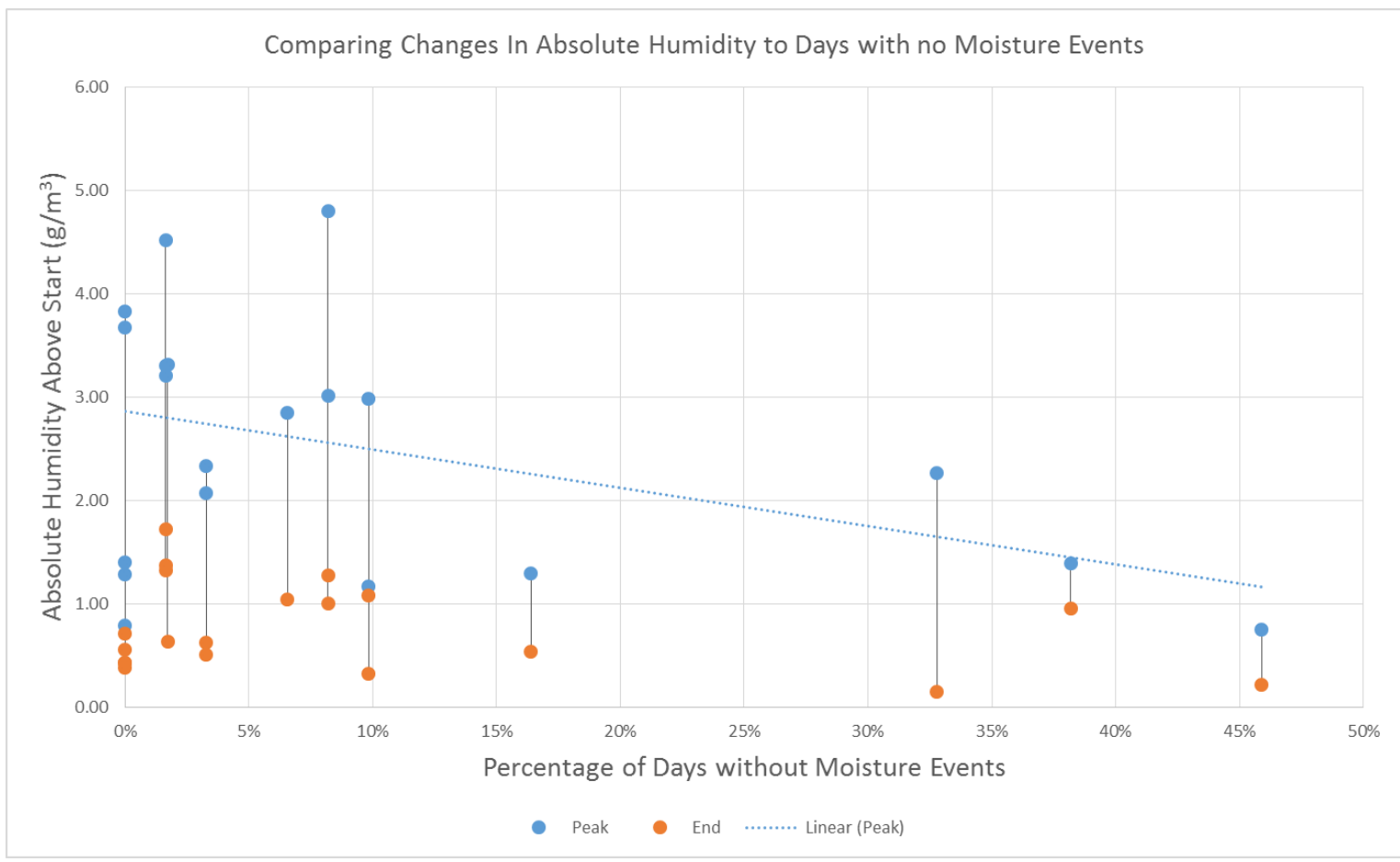

Figure 107 Bathrooms Corrected for Start Absolute Humidity compared to Typical Days with no Moisture Events

Figure 107 compares the changes in median absolute humidity from episode start to peak to the percentage of days over the analysed period which were recorded under typical days as having no moisture events. This appears to indicate a slight trend towards bathrooms with more days that had no moisture events also experiencing smaller changes in absolute humidity from the start of an episode to the peak. However, most houses had less than $10 \%$ of days which met these criteria. Only four houses were outside of this range, so no conclusions can be drawn. 


\subsubsection{Number of Episodes}

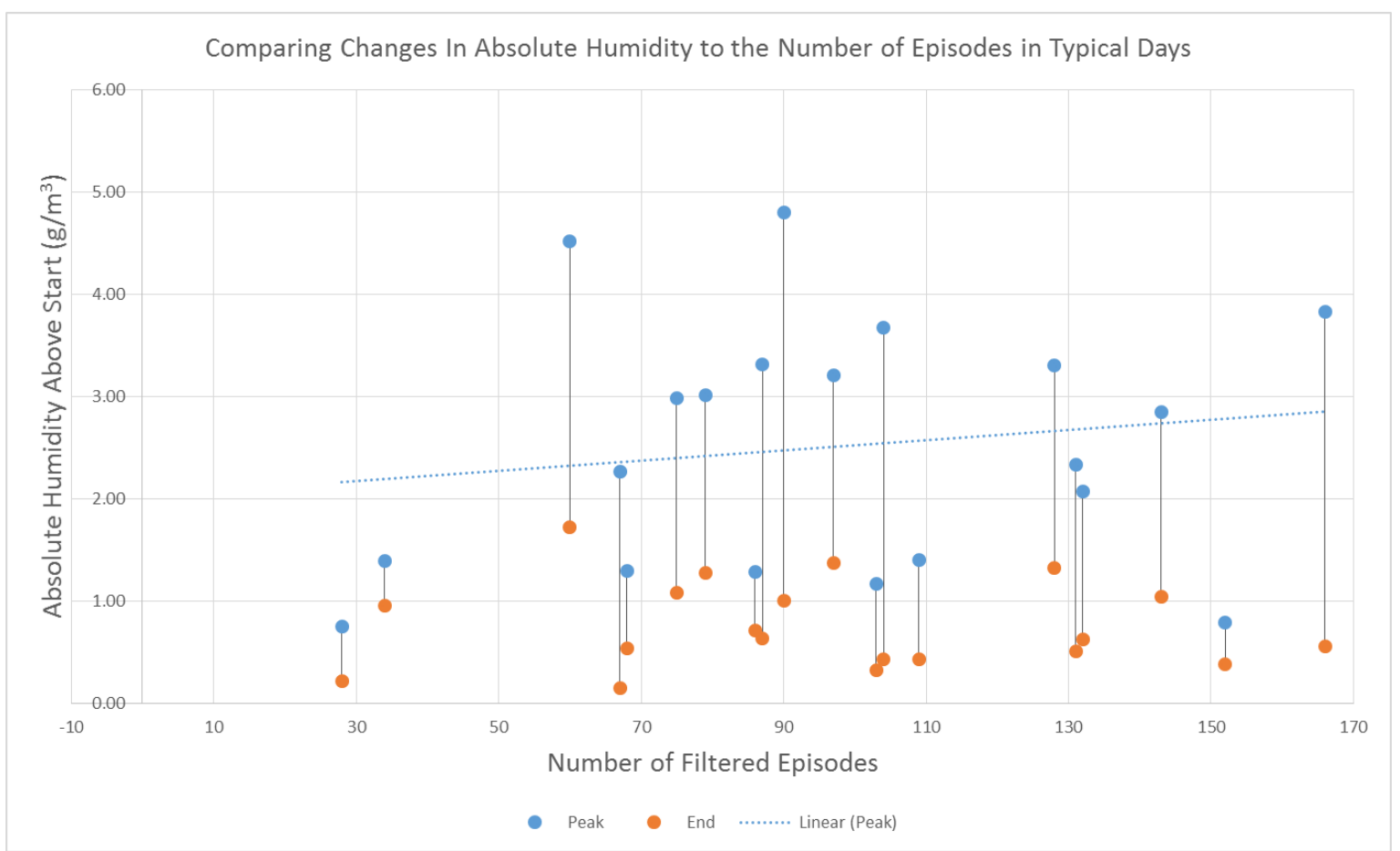

Figure 108 Bathrooms Corrected for Start Absolute Humidity compared to Total Number of Filtered Episodes

Figure 108 compares the changes in median absolute humidity from episode start to peak to the number of episodes recorded for typical days. This used filtered episodes, only counting the larger episodes taking place. There does not appear to be any clear relationship between the number of filtered episodes and the change in absolute humidity from the start of an episode to the peak. 


\subsubsection{Showers/baths per day}

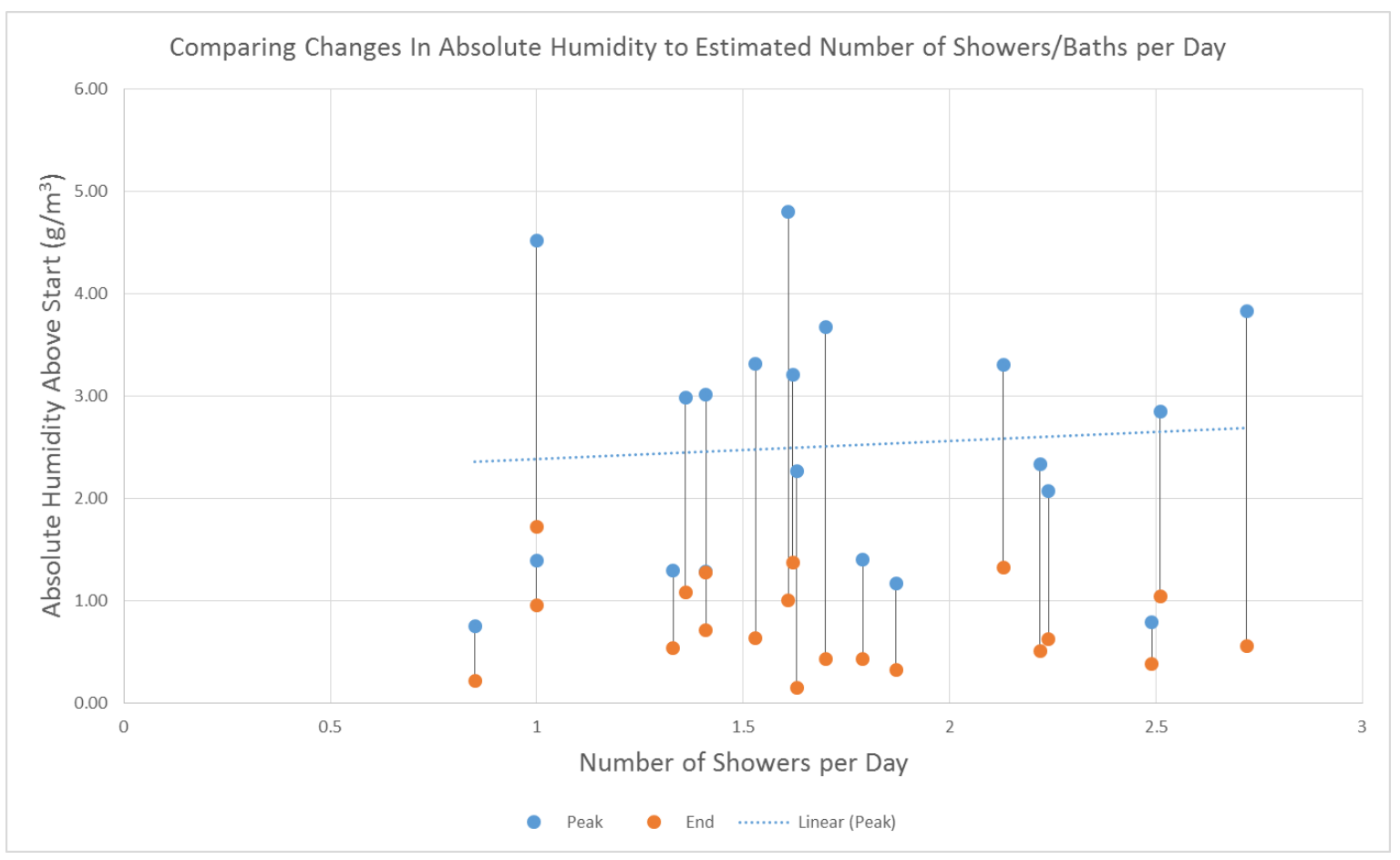

Figure 109 Bathrooms Corrected for Start Absolute Humidity compared to Estimated Showers/baths per day

Figure 109 compares the changes in median absolute humidity from episode start to peak to the estimated number of showers/baths per day (10.2.1 Showers/baths per day per House). There does not appear to be any clear relationship between the estimated number of showers/baths per day and the change in absolute humidity from the start of an episode to the peak. As the number of showers/baths per day is based on a comparison of unoccupied periods (Figure 107) to number of filtered episodes (Figure 108), this further shows that the number of filtered episodes appears to have very little impact on the change in absolute humidity from the start of an episode to the peak. 


\subsection{Location}

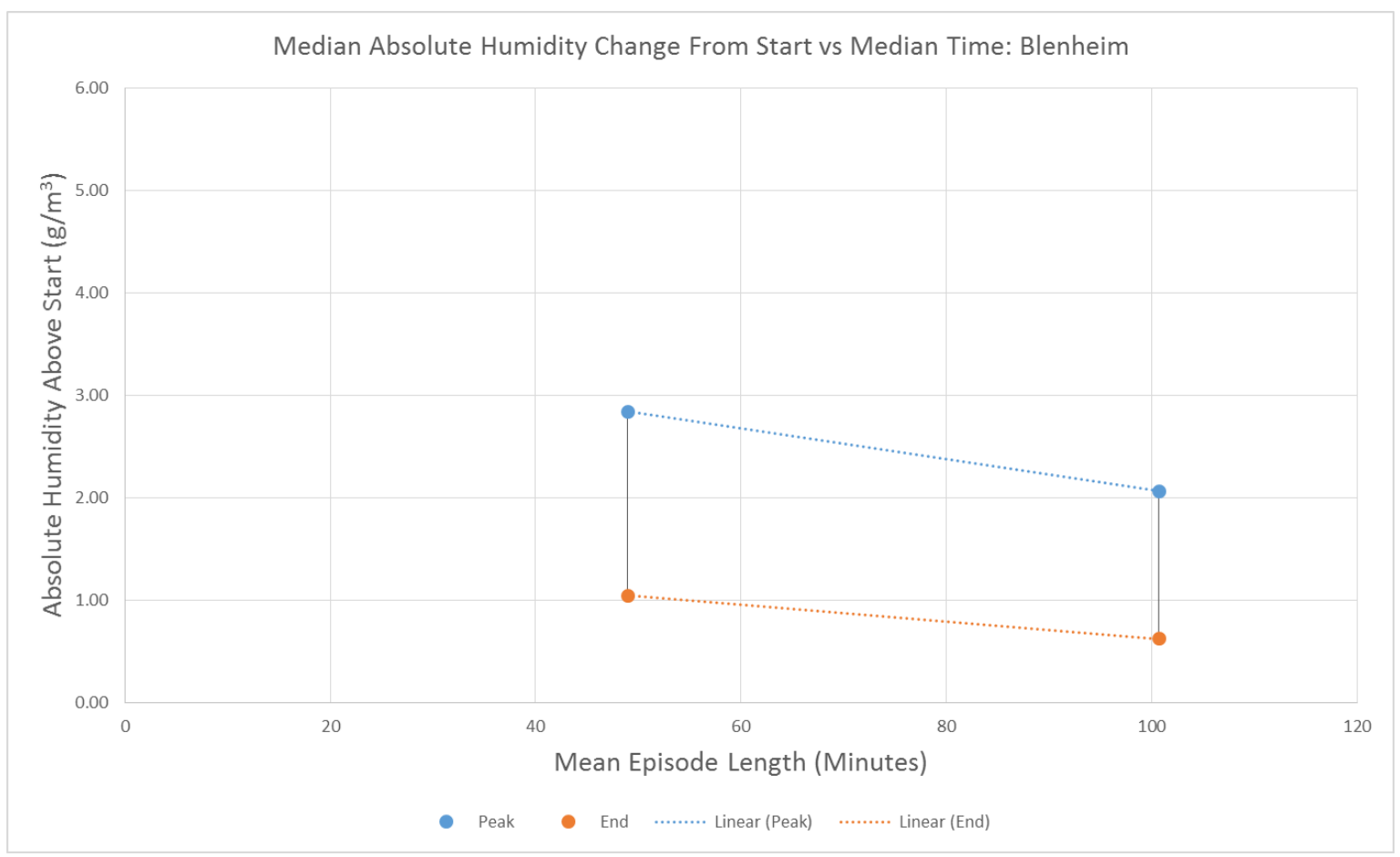

Figure 110 Bathrooms Corrected for Start Absolute Humidity compared to Mean Time: Blenheim

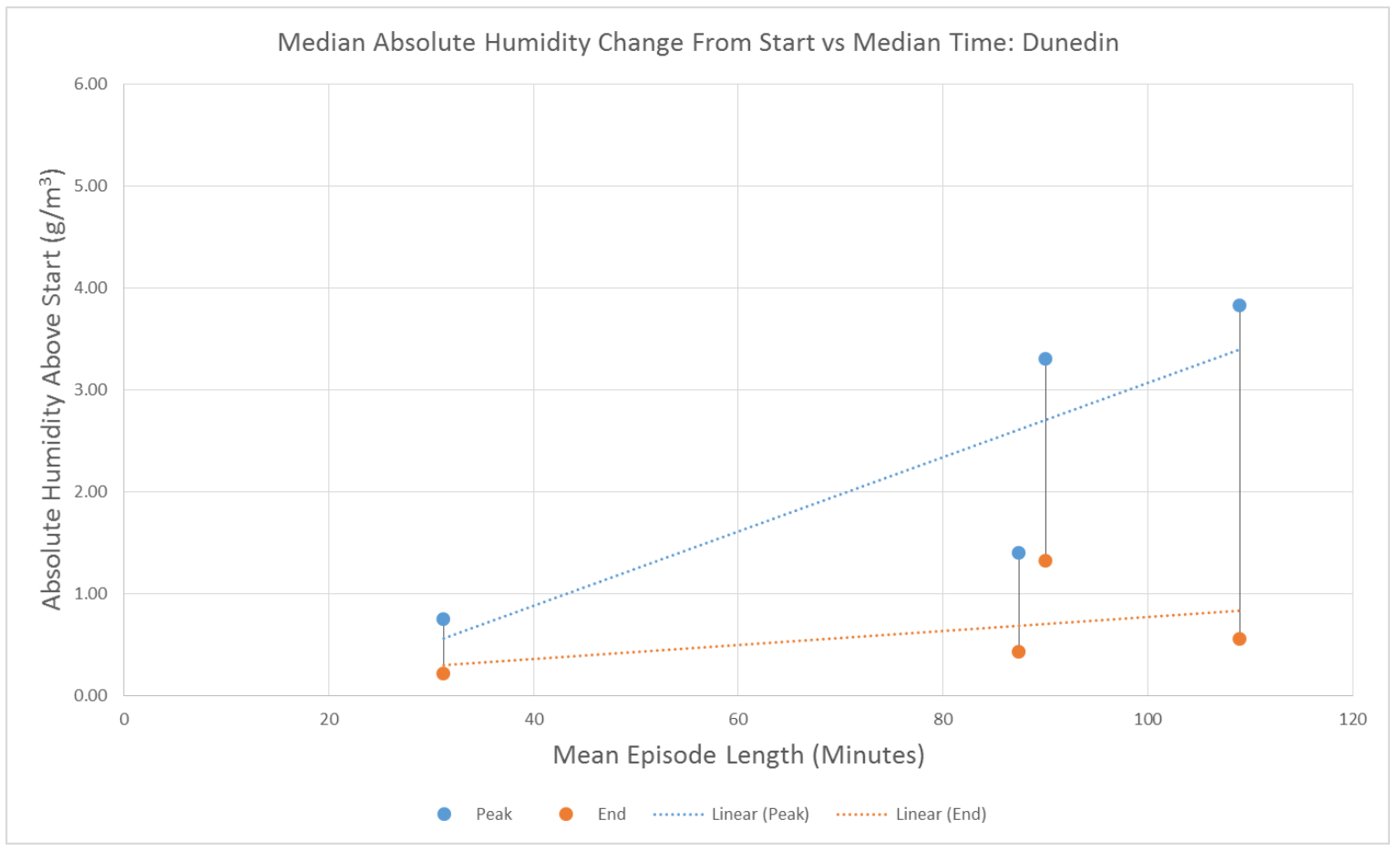

Figure 111 Bathrooms Corrected for Start Absolute Humidity compared to Mean Time: Dunedin

177

Groups and Trends in Data 
Alister Stubbe

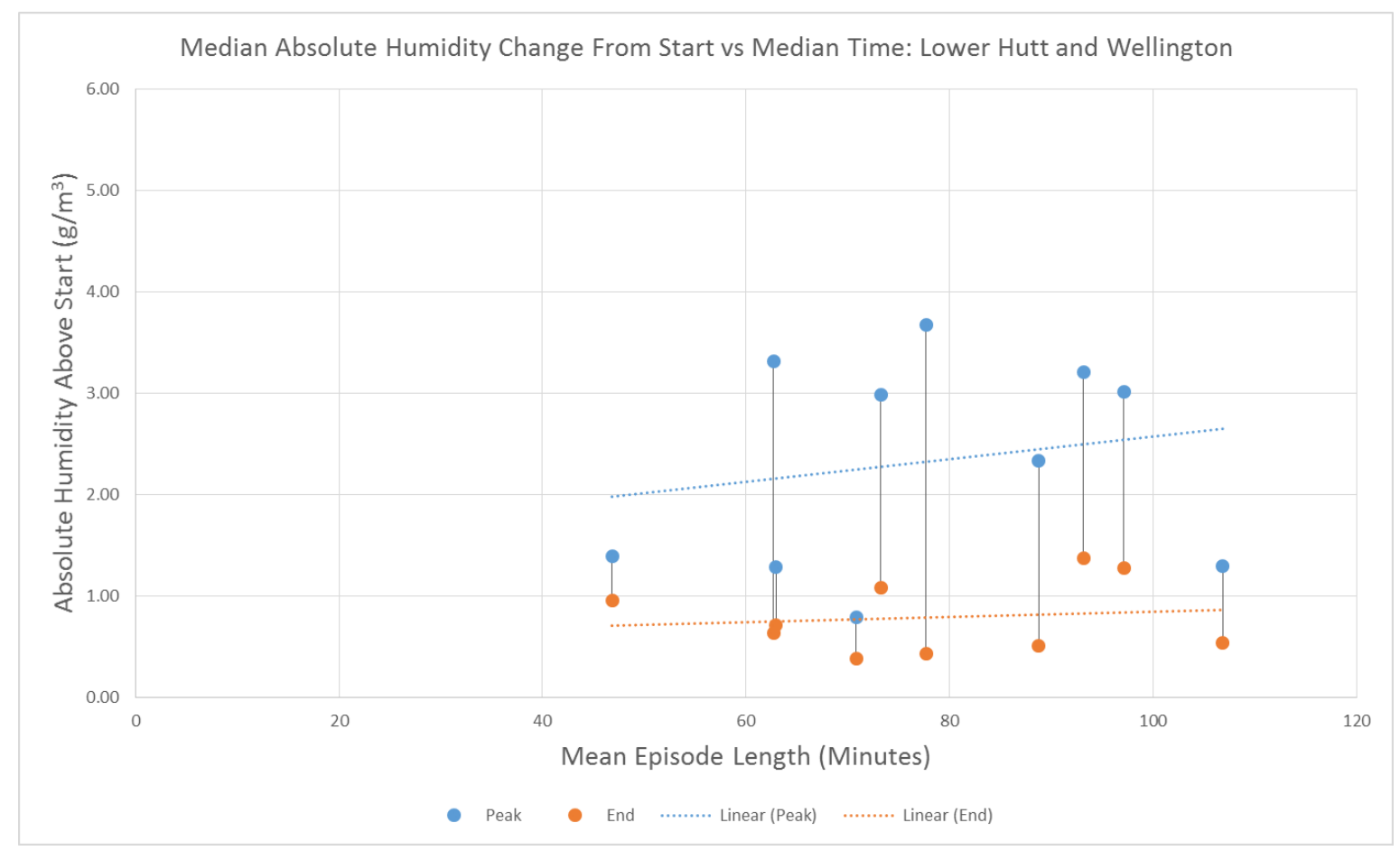

Figure 112 Bathrooms Corrected for Start Absolute Humidity compared to Mean Time: Wellington Region

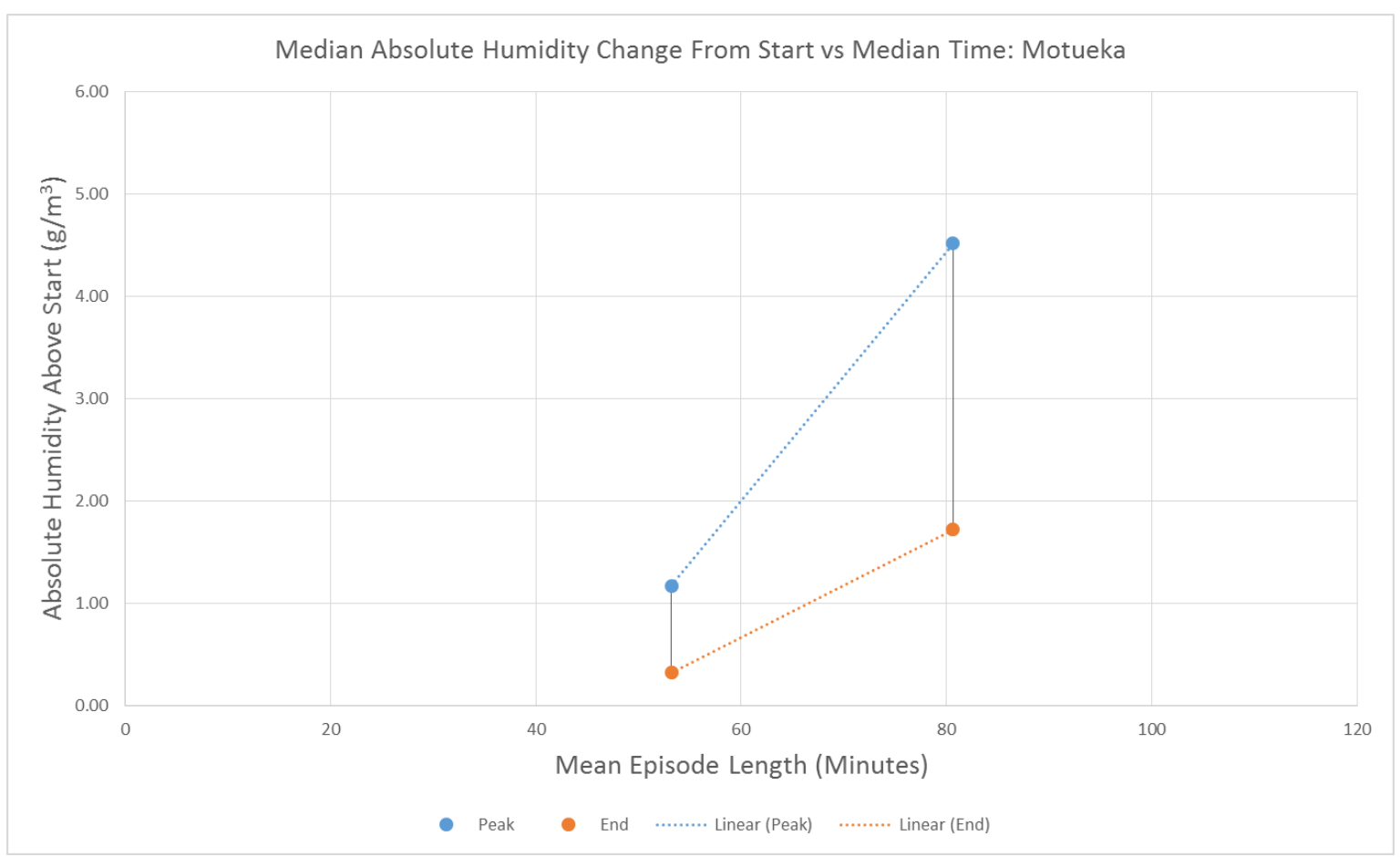

Figure 113 Bathrooms Corrected for Start Absolute Humidity compared to Mean Time: Motueka 


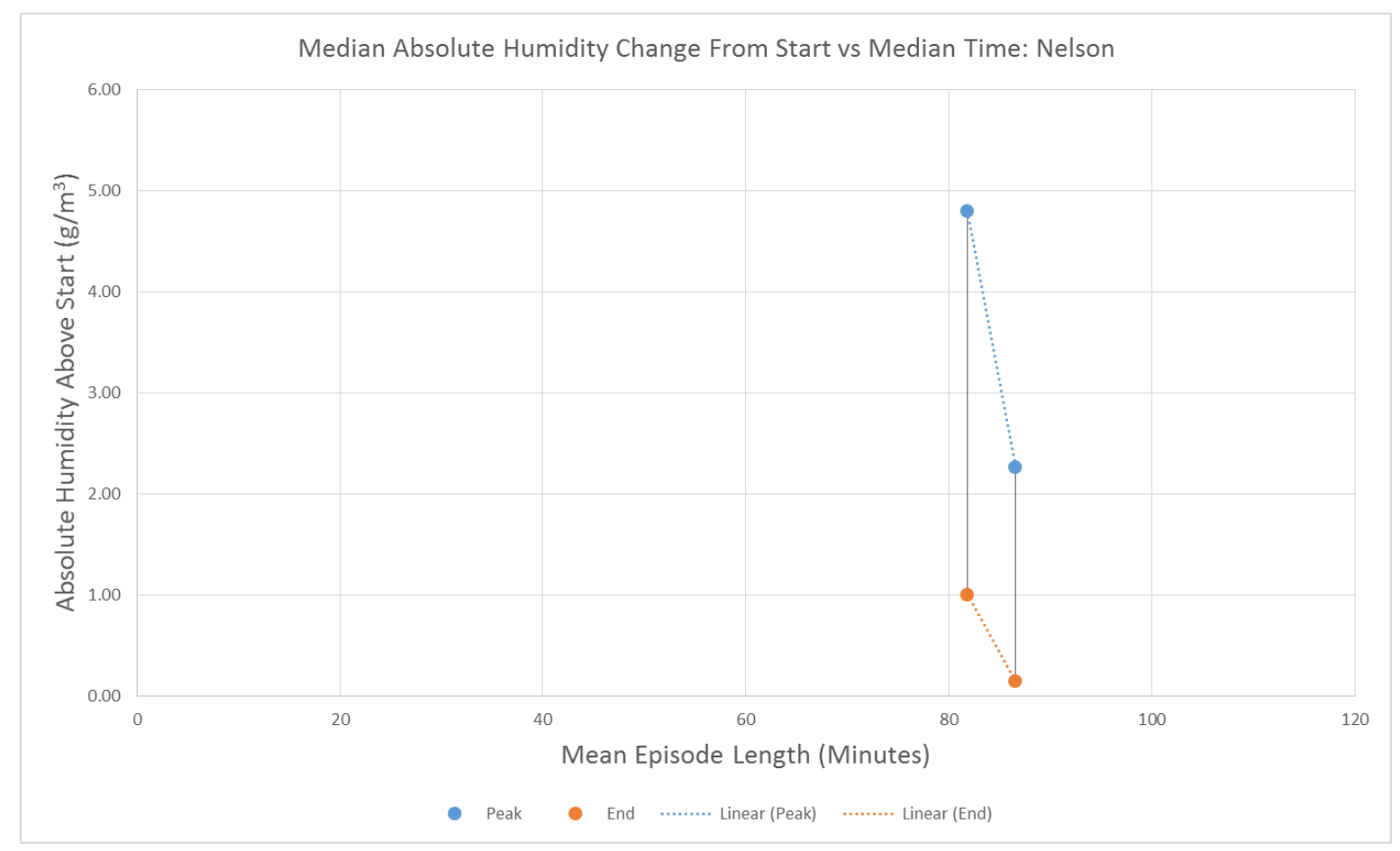

Figure 114 Bathrooms Corrected for Start Absolute Humidity compared to Mean Time: Nelson

The sample size is too small to draw any conclusions about the differences in each bathroom based on region. The only region with more than two houses available information is for Wellington (including Lower Hutt) (Figure 112). For this region the changes in median absolute humidity from episode start to peak varied widely. A larger sample size is required to understand the impact region has on episodes and is likely to require a detailed analysis of the impact of weather. The results of 10.4.2 Median Peak Absolute Humidity suggest that in many cases the region might only have a small impact on the peak absolute humidity reached during an episode. 


\subsection{Presence of a Mechanical Extract Fan}

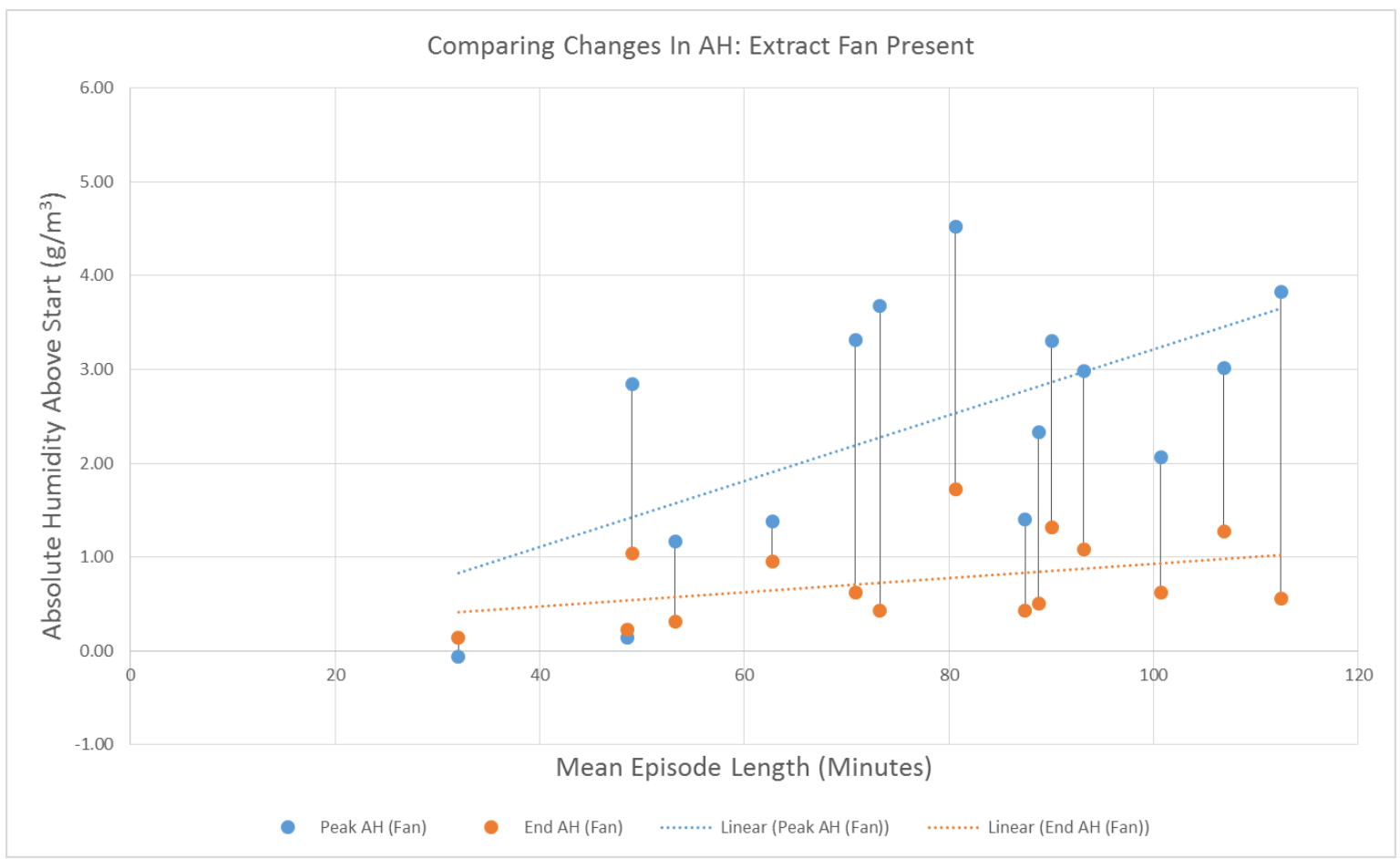

Figure 115 Changes in Absolute Humidity Compared to Start, Bathrooms with and Extract Fan

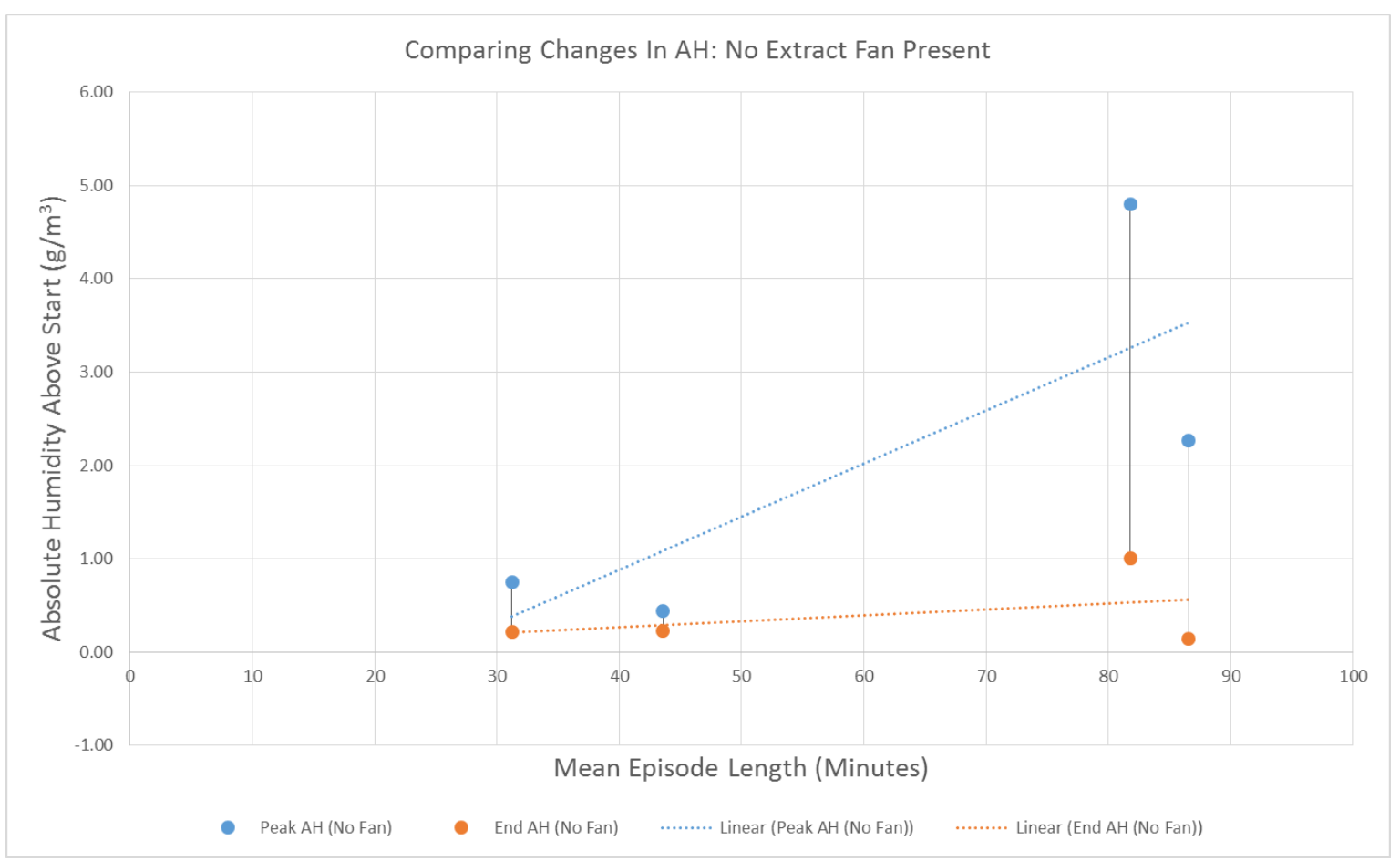

Figure 116 Changes in Absolute Humidity Compared to Start, Bathrooms without an Extract Fan 
Note that second bathrooms (from houses with two bathrooms) are included in this section. There was no information on whether extract fans were present in houses WN3, WN5, WN6, WN8 (both bathrooms WN8.1 and WN8.2), and WN9. Only four of the remaining bathrooms did not have an extract fan present: DN6.1, DN6.2, NL1, and NL2 (Figure 116). All houses had an openable window.

Bathrooms DN6.1 and DN6.2 had among the smallest median peak absolute humidity of any house; $7.90 \mathrm{~g} / \mathrm{m}^{3}$ and $7.97 \mathrm{~g} / \mathrm{m}^{3}$ respectively. These were changes of only $0.75 \mathrm{~g} / \mathrm{m}^{3}$ and $0.45 \mathrm{~g} / \mathrm{m}^{3}$ from the median start absolute humidity respectively. The median peak absolute humidity for NL1 was $9.23 \mathrm{~g} / \mathrm{m}^{3}$, just below the average peak of $10.09 \mathrm{~g} / \mathrm{m}^{3}$ for all bathrooms. This was an increase of $2.26 \mathrm{~g} / \mathrm{m}^{3}$ from the median start absolute humidity, compared to the average change of $2.12 \mathrm{~g} / \mathrm{m}^{3}$ among all houses. By contrast, NL2 had the largest median peak absolute humidity of any house, reaching $13.41 \mathrm{~g} / \mathrm{m}^{3}$, as well as the largest increase from the median absolute humidity with $4.80 \mathrm{~g} / \mathrm{m}^{3}$.

This means that of the three houses without extract fans in bathrooms, one had some of the smallest episodes, one had the largest episodes, and one had fairly average episodes. The sample size is too small to predict what the relationship between episodes and the absence of extract fans (if any) might be. Having such varied results suggests the impact is influenced by other factors (possibly such as house design and occupant behaviour), but there is not enough data available to predict whether this is the case. 
Alister Stubbe 


\section{Chapter 11: Methodology Review}

\subsection{Data Limitations}

\subsubsection{Lack of Building Information}

There was no usable data available on how mechanical ventilation systems were used. Had this been available, it would have greatly contributed towards understanding how absolute humidity was being controlled and whether the occupants were using these systems when required. It would have also provided another way to group houses, showing whether there were any trends in houses with the same ventilation usage. This may help to suggest whether New Zealander's current use of bathroom ventilation is effective, or whether other solutions should be considered. For example, using preventative solutions to stop significant amounts of water being introduced may be an effective way of supplementing insufficient ventilation (van Dijk \& Carson, 2011).

While data was available on window operations, many sensors had been incorrectly installed, resulting in unusable data. Much like the mechanical ventilation system information, understanding how windows were being used would have provided insight both into how moisture events could be controlled, and the impact occupants could have on them.

Information about the house design would have also been useful. There are a variety of other factors present in houses which could have affected measurements. For example, the proximity of the bathroom to other sources of moisture, such as in the kitchen or another bathroom, may have influenced the recorded levels of absolute humidity. This information could have helped identify moisture events that were a result of increases in moisture in another room. A record of factors

\subsubsection{Occupancy and Use}

There was no record of whether houses were occupied during the periods measured. This meant that many assumptions had to be made for interpreting moisture events. Had this 
information been available, any moisture events consistently taking place on days where the house was reported as unoccupied could be assumed to not be a result of occupant activity. Likewise, if days where the house was reported as unoccupied consistently had no moisture event take place, then all moisture events could be assumed to be a result of occupant activity.

There was not information on occupant reported use of baths and/or showers. It has been assumed that the moisture events (specifically episodes) were due to showers, but it is possible that some were baths. It is also possible that use of devices to limit moisture transfer from the shower (e.g. Shower Dome) could also reduce the peak moisture load, but no details were available. This could have been assisted by the availability of digital photographs of each bathroom.

\subsubsection{Weather}

As discussed in the initial data test, comparing the indoor environment of each bathroom to the local weather would have allowed the impact of changes in weather to be analysed. Due to time constraints this was beyond the scope of this study.

The weather data available was measured by NIWA using the recording station nearest to the measured house. Using weather data that was not taken onsite means that these measurements may would not accurately reflect the microclimate influencing the building.

\subsection{Absolute Humidity}

Absolute humidity provided an excellent tool for tracking how much moisture was being introduced to the indoor environment. However, it did not take temperature into account. The amount of moisture air is capable of holding changes with temperature. Thus, an episode that led to an increase of $3 \mathrm{~g} / \mathrm{m}^{3}$ of moisture would have a completely different impact on the bathroom surfaces depending on whether it was hot or cold.

Using a sample of measurements from August and September somewhat helped to mitigate this, limiting the range in temperature a house was likely to experience (compared to the range of temperatures experienced in summer). However, the difference in temperature between night and day could still have had a large impact on results, depending on the level 
of space heating. Comparing a change in absolute humidity in the middle of the day to one in the middle of the night without taking temperature into account does not provide a full understanding of what the indoor environment and surfaces are experiencing.

\subsection{Representative Sample}

As the data used was provided by BRANZ, there was no way to control which houses would be analysed. There was no way of ensuring that these houses represented an appropriate mixture of house designs and locations that was proportional to the types of houses used throughout New Zealand. This means that any conclusions drawn will not necessarily reflect what happens in most New Zealand homes, only a small subset of them. Whether or not these results were representative of the rest of the country was checked by comparing the results to the HEEP study in 10.2 Comparing Results to the Household Energy End-use Project. This demonstrated that the sample size was not large enough.

\subsection{BRANZ Goal}

The initial study carried out on this data (White \& Plagmann, 2017) used aggregate data from all houses to compare median temperature and relative humidity in New Zealand homes. That work focused on how residential houses would perform over the course of an entire day rather than at any specific time. By contrast, this study focussed on periods of rapid change in absolute humidity, analysing how often these periods occurred, how long they lasted, how much moisture they introduced to the space, and how quickly this moisture was removed.

This approach was designed to provide more insight into how New Zealand houses cope during the periods they were most at risk. This information could also be compared to selfreported occupant use of moisture sources such as showers, such as in 10.2 Comparing Results to the Household Energy End-use Project. With a larger sample size, this could provide useful information about the impact occupants have on the indoor environment. 


\subsection{Filtering Episodes}

This study described the indoor environment using two different methods: calculating the mean and median episodes for each house; and identifying the most significant episodes to describe the typical days for each house. Together, the information provided by these two methods allowed the indoor environment to be effectively described and evaluated, showing how the levels of absolute humidity changed during episodes, how long those episodes lasted, and how frequently those episodes would occur. The typical days provided context to the mean and median episode that greatly contributed to their analysis.

However, typical days were based on the full context of each period, including the episode length, the increase/decrease in absolute humidity, potential moisture events, and base moisture levels. This meant the episodes identified using the general definition (7.4.1 Episode) had to be further filtered (7.6.1 Filtering Episodes).

Part of the analysis of moisture events was based on comparing typical days with the mean and median episode information. This mean that information based on filtered episodes was being compared with unfiltered episodes. If episodes had not been filtered, fewer assumptions would need to have been made about how the unfiltered episodes influenced the results. Further research could use the filtered episodes for calculating mean and median information. However, this may result in episodes that had a large impact on the indoor environment being missed. 


\section{Chapter 12: Conclusions}

\subsection{Evaluation of Research Aims and Objectives}

The aim of this study was to identify and analyse periods of rapid change in moisture levels in a set of bathrooms. The study was only partially successful. A method to identify these periods was successfully developed, allowing points of interest in a wide variety of bathrooms to be isolated and used for further research. This was accompanied by a visual representation of key measurements, further assisting comparisons between data sets.

Where this study failed was in providing meaningful insights into what the differences and similarities between periods of interest in each house actually meant. The results simply provide the foundations for further research.

However, that does not mean this study provided no valuable or tangible results in and of itself. Instead, the conclusions that can be drawn focus on the effectiveness of the methods developed to analyse the data. Of particular note was the use of moisture events, episodes, and typical days.

\subsection{Conclusions}

12.2.1 Using Moisture Events as an Indicator of the Indoor Environment

This study was based on identifying and analysing periods of rapid change in absolute humidity. A change of 3\% or higher in absolute humidity between each measurement were categorised as moisture events and used to analyse the indoor environment. This was the first study to attempt this type of analysis. The results are promising, with the method appearing to correctly identify periods where the indoor environment underwent large changes in humidity. Each of these periods were distinct enough to further analyse and group, allowing assumptions to be made about what was happening in the bathroom at the time. 


\subsubsection{Identifying Moisture Events Using Percentage Change}

To identify typical days, every moisture event was manually reviewed (including a total of 2929 episodes across all houses) taking the context of potential moisture events and base moisture levels into account. This process confirmed that the process used for identifying moisture events was successful. A $3 \%$ change in absolute humidity correctly identified periods of rapid change in absolute humidity for every house. To further validate these results, additional information about the indoor environment would be required.

\subsubsection{Episodes}

Categorising moisture events so that similar changes in absolute humidity could be compared was a key component of this study. Identifying episodes allowed the most important sections of moisture events for the analysis (the increase from the start to the peak) to be measured.

Unfortunately basing the analysis on research required several large assumptions. Episodes were assumed to represent showers, baths, or similar moisture sources. There was not enough information collected by the original survey or made available for this research to prove whether this was the case.

\subsubsection{Profiling Houses Using Typical Days}

The information typical days provided about the frequency of different moisture events may be useful for a future study. Several improvements would need to be made to the process before this could be carried out. The existing process was based on filtering episodes to find the largest changes. A better method may be to increase the percentage required for a series of measurements to qualify as a moisture event. This provide a consistent method for identifying the largest moisture events without relying on using other information, making it easier to compare typical days with the other data.

Dividing days into four hour periods may have oversimplified typical days. Smaller period (such as one hour periods) would provide more accurate information that could be generalised if required. This would allow a more thorough analysis to be performed.

\subsubsection{Mean vs Median Results Analysis}

The median measurements of episodes were used throughout the analysis. Episodes were found to vary widely in size. As a result, the mean measurements would often represent the 
midpoint of two extremely different episodes, failing to represent what took place in the indoor environment. The median provided a far better measurement, representing the most common type of episodes while ignoring outliers. However, these outliers were still important and likely played an important role in the indoor environment. Further categorising episodes and using the mean measurement of each of these for analysis would provide far better insight into the indoor environment.

\subsubsection{Comparing Results to Household Energy End-use Project}

The HEEP study provided a means of validating results, checking whether the measurements of moisture events were similar to self-reported information about shower usage. While the results were promising, it demonstrated that the sample size was too small to make significant claims about the behaviour of moisture sources in New Zealand bathrooms.

Future measurements should be taken at smaller intervals to better measure the length of time moisture is being introduced to the indoor environment.

\subsubsection{Impact of Absolute Humidity at the Start of Episodes}

The absolute humidity at the start of an episode was found to have very little impact on the absolute humidity at the peak of an episode. This means the indoor environment has almost no impact on an episode; the bathroom conditions before the episode began do not appear to be a significant factor in the amount of moisture introduced. This is likely because an episode will introduce enough moisture for the air to reach near saturation. Once it reaches this point, it does not matter how much water was in the air to begin with. Further studies should measure both relative humidity and absolute humidity during moisture events to demonstrate whether this is the case.

\subsubsection{The Impact of Multiple Bathrooms}

This was one of the most interesting results of the study. Houses with two bathrooms were found to behave differently to houses with only one bathroom. Moisture episodes were found to be different for each bathroom, with the changes in absolute humidity being significantly smaller in one of them. This was found to be the case for all every house with two bathrooms. This highlights the impact of occupant behaviour on moisture episodes.

\subsubsection{Bathroom Area}

As expected, larger rooms were measured to have lower levels of absolute humidity. Interestingly though, the smallest bathrooms also had low levels of absolute humidity. As 
absolute humidity is a measure of grams per cubic metre, this result seems counterintuitive. This may be due to different (or fewer) moisture sources being present. It might also be caused by occupants acting more aware of the impact moisture can have, especially in a small room that can easily be flooded with moisture.

\subsection{Further Research}

\subsubsection{Additional Data}

This study demonstrated that analysing moisture events in residential bathrooms can provide useful information about the indoor environment. Supplementing this analysis with more information, as discussed in 6.7 Results for Additional Consideration, would allow trends in measurements to be analysed in greater depth. Increasing the sample size would also provide more meaningful results, such as how region affects moisture events.

\subsubsection{Additional Research on Multiple Bathrooms}

This seems to be the most promising result of this study, with episodes behaving completely differently when two bathrooms are present. The exact cause of this should be further studied, specifically whether occupant behaviour changes when more than one bathroom is available. This may provide useful information on promoting better moisture control.

\subsubsection{Other Areas of Houses}

This study focused on the bathroom, although other areas of the house (notably the kitchen) are also at risk from moisture. Comparing moisture events from different areas of the house would provide useful information, particularly on occupant behaviour.

Differences between weekdays, weekends, and unoccupied periods could also be compared to help better understand the impact occupants are having on the property.

\subsubsection{Impact of Occupant Control}

Houses could also be compared based on the level of occupant control. For example, how do residential bathrooms with automatic extractor fans linked to lights compare to residential bathrooms with extractor fans that must be manually switched on? How may this vary once occupants are taught about the importance of removing moisture? Does this increase the performance of bathrooms with manual systems and, if so, is this increase permanent or only temporary? By contrast, do occupants not explicitly told how to keep 
Moisture in New Zealand Bathrooms

their bathroom free of moisture have the potential to create an unhealthy environment if they do not understand the full impact of their actions? This may provide useful information for improving the NZBC. 
Alister Stubbe 


\section{Chapter 13: References}

Andersen, R., Fabi, V., Toftum, J., Corgnati, S. P., \& Olesen, B. W. (2013). Window opening behaviour modelled from measurements in Danish dwellings. Building and Environment, 69, 101-113. https://doi.org/10.1016/j.buildenv.2013.07.005

Annila, P. J., Hellemaa, M., Pakkala, T. A., Lahdensivu, J., Suonketo, J., \& Pentti, M. (2017). Extent of moisture and mould damage in structures of public buildings. Case Studies in Construction Materials, 6, 103-108. https://doi.org/10.1016/j.cscm.2017.01.003

Barnard, L., \& Zhang, J. (2016). The Impact of Respiratory Disease in New Zealand: 2016 Update. University of Otago. Retrieved from https://s3-ap-southeast-

2.amazonaws.com/assets.asthmafoundation.org.nz/documents/REPORT-The-impact-onrespiratory-disease-in-New-Zealand-2016-update.pdf

Bassett, M. R. (2011). Residential Moisture Presentation. Presented at the BRANZ Conference.

Bekö, G., Lund, T., Nors, F., Toftum, J., \& Clausen, G. (2010). Ventilation rates in the bedrooms of 500 Danish children. Building and Environment, 45(10), 2289-2295. https://doi.org/10.1016/j.buildenv.2010.04.014

Camilleri, M., Burrough, L., Pollard, A., Saville-Smith, K., \& Fraser, R. (2010). Energy Use in New Zealand Households - Final Report on the Household Energy End-use Project (pp. 256257). Judgeford, New Zealand: BRANZ. Retrieved from https://www.branz.co.nz/cms_show_download.php?id=a9f5f2812c5d7d3d53fdaba15f2c14 d591749353

Canada Mortgage and Housing Corporation. (2004). Analysis of Ventilation System Performance in New Ontario Houses.

Fabi, V., Andersen, R. V., Corgnati, S., \& Olesen, B. W. (2012). Occupants' window opening behaviour: A literature review of factors influencing occupant behaviour and models. Building and Environment, 58, 188-198. https://doi.org/10.1016/j.buildenv.2012.07.009 Humidity Conversion Formulas: Calculation Formulas for Humidity. (2013). Vaisala Oyj. 
Leardini, P. M., \& Van Raamsdonk, T. (2010). Design for airtightness and moisture control in New Zealand housing. In Proceedings of the New Zealand Sustainable Building Conference, Wellington, New Zealand (Vol. 2628). Retrieved from http://www.irbnet.de/daten/iconda/CIB18068.pdf

Mahooti-Brooks, N., Storey, E., Yang, C., Simcox, N. J., Turner, W., \& Hodgson, M. (2004). Characterization of Mold and Moisture Indicators in the Home. Journal of Occupational and Environmental Hygiene, 1(12), 826-839. https://doi.org/10.1080/15459620490890332 McDowall, P. (2017). Open Windows for Dry Home. Build, (158), 84-85.

McNeil, S. (2016). Combating Internal Moisture. Build, (151), 46-48.

McNeil, S., Plagmann, M., McDowall, P., \& Bassett, M. R. (2015). The Role of Ventilation in Managing Moisture inside New Zealand Homes (Study Report No. 341). BRANZ.

McNeil, S., Quaglia, L., Plagmann, M., \& Overton, G. (2011). A Survey of Airtightness and Ventilation Rates in Post 1994 NZ Homes. BRANZ.

McNeil, S., \& Rupp, S. (2017, March). BRANZ Ventilation Seminar. Wellington. Ministry of Business, Innovation \& Employment. (2014, February 14). Building Code Handbook 3rd Edition. Amendment 13.pdf.

O’Brien, W., \& Gunay, H. B. (2014). The contextual factors contributing to occupants' adaptive comfort behaviors in offices - A review and proposed modeling framework. Building and Environment, 77, 77-87. https://doi.org/10.1016/j.buildenv.2014.03.024 Pekkanen, J., Hyvarinen, A., Haverinen-Shaughnessy, U., Korppi, M., Putus, T., \& Nevalainen, A. (2007). Moisture damage and childhood asthma: a population-based incident casecontrol study. European Respiratory Journal, 29(3), 509-515. https://doi.org/10.1183/09031936.00040806 Popenhagen, R. (2012, May). Moisture and Ventilation: What can go Wrong! Presented at the BOINZ Conference, Auckland. Retrieved from http://www.ecodesignadvisor.org.nz/assets/Uploads/1-1274472-MoistureandVentilationHowwegetitwrongBOINZconferenceAuckland15May2012x.pdf 
Moisture in New Zealand Bathrooms

Standards New Zealand. (1990, September 13). NZS 4303:1990 Ventilation for Acceptable Indoor Air Quality.pdf.

Straube, J. F. (2002). Moisture in buildings. ASHRAE Journal, 44(1), 15.

Su, B. (2006). Prevention of Winter Mould Growth in Housing. Architectural Science Review, 49(4), 385-390. https://doi.org/10.3763/asre.2006.4950

van Dijk, L., \& Carson, J. K. (2011). Assessment of the performance of the Showerdome ${ }^{T M}$ device. The University of Waikato. Retrieved from https://showerdome.co.nz/assets/uploads/2016/11/waikato-university-report.pdf White, V., \& Jones, M. (2015). Warm, Dry Healthy? Insights from the 2015 House Condition Survey on Insulaton, Ventilation, Heating and Mould in New Zealand Houses. Judgeford, New Zealand: BRANZ. Retrieved from https://www.branz.co.nz/cms_show_download.php?id=50335e67bb00f3e0464097be1d4d7 $1 \mathrm{ac} 8 \mathrm{a} 85 \mathrm{f} 6 \mathrm{bf}$

White, V., \& Plagmann, M. (2017, April 1). Bathroom Habits Falling Short. BRANZ. Retrieved from https://www.buildmagazine.org.nz/articles/show/bathroom-habits-falling-short

Yik, F. W. H., Sat, P. S. K., \& Niu, J. L. (2004). Moisture Generation through Chinese Household Activities. Indoor and Built Environment, 13(2), 115-131.

https://doi.org/10.1177/1420326X04040909

Zhang, Y., \& Barrett, P. (2012). Factors influencing the occupants' window opening behaviour in a naturally ventilated office building. Building and Environment, 50, 125-134. https://doi.org/10.1016/j.buildenv.2011.10.018 